PB $257 \quad \ldots 54$

\title{
IN SITU IMPULSE TEST AN EXPERIMENTAL AND ANALYTICAL EVALUATION OF DATA INTERPRETATION PROCEDURES
}

Shannon \& Wilson, Inc. and Agbabian Associates

for

U.S. Nuclear Regulatory Commission 


\section{DISCLAIMER}

This report was prepared as an account of work sponsored by an agency of the United States Government. Neither the United States Government nor any agency Thereof, nor any of their employees, makes any warranty, express or implied, or assumes any legal liability or responsibility for the accuracy, completeness, or usefulness of any information, apparatus, product, or process disclosed, or represents that its use would not infringe privately owned rights. Reference herein to any specific commercial product, process, or service by trade name, trademark, manufacturer, or otherwise does not necessarily constitute or imply its endorsement, recommendation, or favoring by the United States Government or any agency thereof. The views and opinions of authors expressed herein do not necessarily state or reflect those of the United States Government or any agency thereof. 


\section{DISCLAIMER}

Portions of this document may be illegible in electronic image products. Images are produced from the best available original document. 


\section{NOTICE}

This report was prepared as an account of work sponsored by the United States Government. Neither the United States nor the United States Nuclear Regulatory Commission, nor any of their employees, nor any of their contractors, subcontractors, or their employees, makes any warranty, express or implied, nor assumes any legal liability or responsibility for the accuracy. completeness or usefulness of any information, apparatus, product or process disclosed, nor represents that its use would not infringe privately owned rights. 


\section{IN SITU IMPULSE TEST AN EXPERIMENTAL AND ANALYTICAL EVALUATION OF DATA INTERPRETATION PROCEDURES}

Manuscript Completed: August 1975

Date Published: September 1976

Shannon \& Wilson, Inc. and Agbabian Associates

Seattle, Washington 98103

EI Segundo, California 90245

Prepared for the U.S. Nuclear Regulatory Commission

Under Contract No. AT(04-3)-954

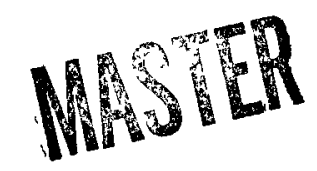


•

- 


\section{ABSTRACT}

special experimental field testing and analytical studies were undertaken at Fort Lawton in Seattle, Wasington, to study "close-in" wave propagation and evaluate data interpretation procedures for a new in situ impulse test. This test was developed to determine the shear wave velocity and dynamic modulus of soils underlying potential nuclear power plant sites. The test is different from conventional geophysical testing in that the velocity variation with strain is determined for each test. In general, strains between $10^{-1}$ and $10^{-3}$ percent are achieved.

The experimental field work consisted of performing special tests in a large test sand fill to obtain detailed "close-in" data. Six recording transducers were placed at various points on the energy source, while approximately 37 different transducers were installed within the soil fill, all within 7 feet of the energy source. Velocity measurements were then taken simultaneously under controlled test conditions to study shear wave propagation phenomenology and help evaluate data interpretation procedures. Typical test data are presented along with detailed descriptions of the results. These data show that both compression and shear waves separate into two distinct repeatable waves at a distance of about 1.8 feet from the energy source. Beyond this point, wave characterististics such as amplitude, frequency, and arrival times of both $\mathrm{P}$ and $\mathrm{S}$ waves can be studied as the wave travels outward into the surrounding soil mass.

The analytical work supported the field experimentation with calculations to simulate the same "close-in" conditions and results of the experimental program. Both linear elastic and non-linear finite element calculations were carried out under this program, in which different interpretation procedures were applied to the results, in order to establish the most suitable procedure.

Additional tests were also performed to study repeatability, effects of symmetry and "shadow", prior load history, variations in hammer weight and drop height, jacking pressure, casing, P-wave, and anchor coupling. 
• 
This report presents the results of combined experimental and analytical studies undertaken to continue development of an in situ impulse test for the determination of the soil shear modulus. Emphasis of the field work was directed toward making simultaneous "close-in" field measurements at various points in the soil surrounding the test's energy input source. From these data, test characteristics, general soil behavior, and the wave propagation phenomenon could be studied under ideal test conditions and at shear strains in the strong motion earthquake range. The analytical work was oriented toward supporting the field effort through evaluation of the experimental results combined with additional calculations to study the same close-in behavior as the experimental test program. Both sets of results could then be compared and together provide insight into data interpretation procedures for this test. This work represents a part of continuing studies to evaluate in situ soil behavior under earthquake loading conditions. This and the other related studies each provide important steps in the overall project for determination of the best methods of evaluation and prediction of soil behavior of potential nuclear power plant sites under seismic loading conditions.

This is the fifth report prepared by the joint venture of Shannon \& Wilson, Inc., (SW), and Agbabian Associates, (AA), on the in situ test development project. The current work was conducted under contract No. AT (04-3)-954 between the joint venture (SW-AA) and the Nuclear Regulatory Commission.

A number of contributions by both members of the joint venture assisted in conducting these research studies and in the preparation of this report. For Shannon \& Wilson, Inc. the primary contributors were Mr. S. D. Wilson, Mr. F. R. Brown and Mr. J. H. Troncoso. Dr. R. P. Miller was the Project Engineer 
for Shannon \& Wilson and is also the Project Manager for the joint venture. For Agbabian Associates, Mr. S. D. Werner was the Project Engineer, assisted by Mr. D. Van Dillen and Mr. K. Boisen. Dr. Lydik S. Jacobsen, special consultant to the joint venture, also provided valuable guidance in these studies. 
1.1 STATEMENT OF PROBLEM

1.2 APPROACH TO WHE PROBLEI 2

1.3 SCOPE 5

2

IN SITU IMPULSE TEST

2.1 TEST DESCRIPTION 9

2.2 TEST EQUIPMENT 10

2.2.1 Anchor and Hammer Assembly 10

2.2 Sensors 14

2.2.3 Recording Equipment 15

2.2.4 Surveying Equipment 18

$\begin{array}{lll}2.3 & \text { FIELD EXPLORATIONS AND TESTING } & 19 \\ \text { PROCEDURES } & \end{array}$

2.3.1 Field Exploration and Surveying Procedures 20

2.3.2 Field In Situ Testing 22

2.4 DATA PROCESSING 24

2.4.1 Initial Checkout Phase 25

2.4.2 Computer Processing Phase 27

2.4.3 Determination of VelocityStrain Relationship

3 ANALYTICAL DESCRIPTION OF THE IN SITU IMPULSE TEST $\quad 36$

3.1 ANALYSIS OF TEST CONDITIONS 36

3.1.1 Conditions Caused by Stress Relief 36

3.1.2 Disturbances Caused by Drilling 39

3.1.3 Conditions Caused by Coupling 40

3.2 ANALYSIS OF THE GENERATING STATION 43

3. 3 DISCUSSION OF WAVE PROPAGATION PHENOMENA 
FINITE ELEMENT ANALYSES 50

4.1 INTRODUCTION 50

4.1.1 Purpose of Calculations 50

4.1.2 Scope of Calculations 52

4.2 FINITE ELEMENT MODEL 53

4.2.1 Finite Element Grid 54

4.2.2 Application of Dynamic Loading 56

4.2.3 Representation of Soil

Materials

4.2.4 Integration Time step 59

4.3 RESULTS OF ANALYSIS 60

4.3.1 Comparison with Experiment 61

4.3.2 General Nature of Waves

Generated During In Situ Soil

Tests

67

4.3.3 Assessment of Data Processing

Procedures for Determination

of In Situ Shear Wave Velocities 72

4.3.4 Assessment of Data Processing

Procedures for Determination

of In Situ Shear Strain 85

4.3.5 Evaluation of Total Data

Reduction Systems 90

4.4 CONCLUSIONS

99

5 EXPERIMENTAL STUDY OF WAVE PROPAGATION

IN AN ARTIFICIAL SOIL FILL 103

5.1 TEST DESCRIPTION 103

5.2 WAVE PROPAGATION CHARACTERISTICS 107

$\begin{array}{ll}\text { 5.2.1 Particle Velocity } & 107 \\ & \text { Characteristics }\end{array}$

5.2.2 Displacement Characteristics 112

5.3 SHEAR WAVE VELOCITY IN FREE FIELD SOIL

116

5.3.1 Velocities from Waveforms 117

5.3.2 Comparison of Several Methods

for Determining Velocity 119

5.4 SHEAR STRAINS IN FREE FIELD SOILS 124

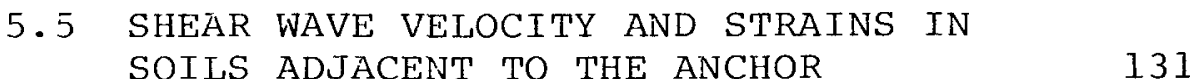


5.5.1 Anchor Records

j.5.2 Dimensional Analysis

Procedures

135

5.5.3 Shear Wave Velocity at Anchor 136

5.5.4 Shear Strain at Anchor 140

6 PRODUCTION TEST DATA INTERPRETATION

PROCEDURES

142

6.1 FREE-FIELD STATIOISS

142

6.2 ANCHOR STATION

143

6.3 FUTURE WORK

145

REFERENCES

146

APPLNDTX A

DESCRIPTION OF NONLINEAR SOIL MODEL

A-1

APPENDIX B

PNEUMATIC ANCHOR RESULTS

B-1

APPENDIX C

SOIL PROPERTIES

$\mathrm{C}-1$

APPENDIX D

FILL CONSTRUCTION AND EQUIPMENT

INSTALLATIOIJ AT FORT LAWTON

$\mathrm{D}-1$

APPENDIX E

T'RANSDUCER SPACING CRITERIA FOR

SPECIAL TESTS

$\mathrm{E}-1$

APPENDIX F

STATISTICAL ASSESSMENT OF SPECIAL

TEST RESULTS

$\mathrm{F}-1$

APPEIJDIX G

GENERAL ASSESSMENT OF SPECIAL

TEST RESULTS

$\mathrm{G}-1$ 
IN SITU IMPULSE TEST

AN EXPERIMENTAL AND ANALYTICAL

EVALUATION OF

DATA INTERPRETATION PROCEDURES

CHAPTER 1

INTRODUCTION

\subsection{STATEMENT OF PROBLEM}

As more sophisticated analytical techniques are developed to predict soil behavior under seismic loading conditions, more accurate input parameters must likewise be provided. One of the more important of these properties is the dynamic shear modulus. This is determined either in the laboratory or in situ, and its numerical value varies not only with the soil type and its physical characteristics but also with the magnitude of the imposed vibratory forces. The procedures for these existing tests together with the limitations of each test are described in a previous report (SW-AJA, 1972).

In situ measurements presently are made at strains smaller than those produced by earthquakes, while conventional laboratory tests are performed at strains either higher or lower than those produced by most earthquakes. However, laboratory results may be complicated by sample disturbance, imperfect duplication of in situ stress conditions, and effects of stress history, structure and cementation. In either case, extrapolation of the measured data is required to adjust the modulus to the strains of interest. To assure that laboratory values of moduli are consistent with field values during large earthquakes and that extrapolation of test data is correctly done, there existed a need to develop an in situ test procedure to obtain moduli data at frequent depth intervals and at strains in the strong motion earthquake range. 
To address this problem, a new in situ impulse test procedure has been developed (SW-AA, 1974). This test employs a cross-hole wave propagation procedure with velocity sensors located in closely spaced adjacent borings. A schematic drawing of the basic system is shown in Fig. 1-1. The signal generating system consists of an in-hole anchor and hammer assembly which imparts an impulsive shearing load to the soil. The shape of the generated pulse is controlled by a spring located between the anchor system and the hammer, and the magnitude by the mass of the hammer and the height of drop.

With this new procedure, consistent repeatable test data at the strains of interest were obtained on a production basis from velocity transducers positioned on the anchor and in the free field soil in adjacent borings. Studies of this data provided reasonably rational procedures for interpreting the results even though all aspects of the test were not clear. However, further attempts to understand 1$)$ the wave propagation phenomenon between the energy source and the free field sensors, 2) the motions of the anchor, and 3) the cause of minor distortions in the recorded velocity signatures revealed the "real" complexities in this seemingly simple test procedure. Therefore to provide the best and most rational interpretation of future test results, further research to develop a better understanding of the test and the data obtained was considered necessary.

\subsection{APPROACH TO THE PROBLEM}

To further evaluate the details of the test procedure, a research program was first developed. This program approached the problem both theoretically and experimentally. From available literature, closed form solutions of idealized cases of the test setup were reviewed primarily to establish likely attenuation rates in the near vicinity of the anchor. This was then followed by two numerical computations of the "close-in" test setup using 


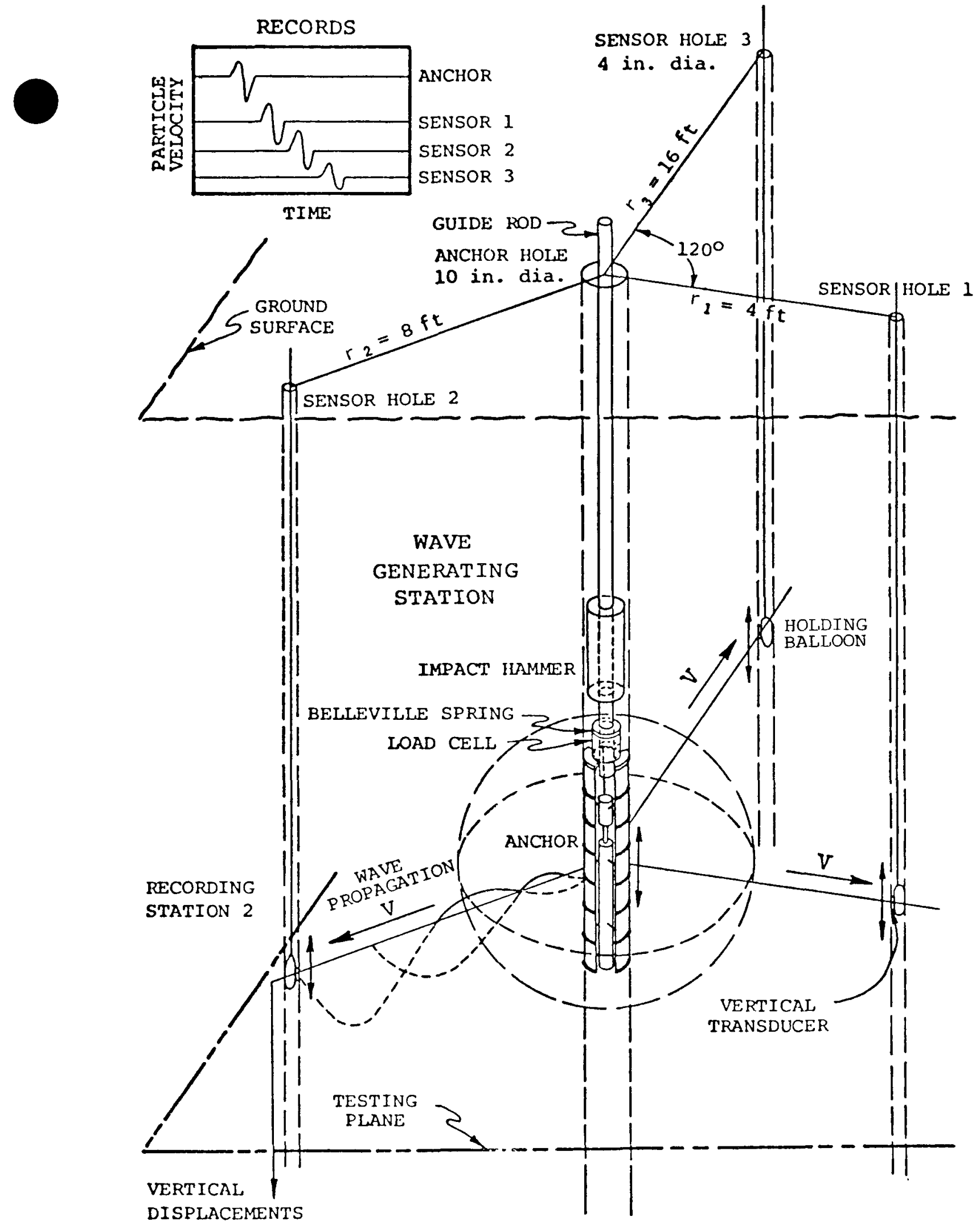

FIGURE $1-1$ SCHEMATIC REPRESENTATION OF IN SITU IMPULSE TEST 
finite element procedures. The first calculation was performed using linear elastic soil properties. This work was then followed by one using non-linear properties. The primary purpose of these calculations was to evaluate procedures for obtaining in situ soil properties from the test data. While these calculations cannot duplicate field test conditions exactly, they nevertheless provide important information which is used to assist in understanding the entire problem. The primary advantage of such calculations is that the input material properties provided a consistent basis for evaluating those properties obtained by applying various data processing techniques to the node point velocity histories.

A second phase of this program consisted of the measurement of detailed test data from additional tests conducted under controlled conditions in an artificial fill, constructed and instrumented for this work. This silty sand fill was placed in a 10-foot deep by 30-foot diameter pit, and manually compacted around the wave-generating station (anchor and hammer assembly) and 32 receiving stations. These stations, consisting of vertically and horizontally-oriented velocity transducers and accelerometers, were laid out in select configurations at different elevations all within seven feet from the energy source. Simultaneous tests consisting of four measurements at a time were accomplished under constant test conditions such that the propagating pulse could be studied as it travels from the source through the free-field soil. Of particular concern was the appropriate treatment of the velocity record obtained from the transducer mounted on the anchor. This record is unlike the free field sensor records in that it represents the motion of the anchor or a part of it and not necessarily the motion of the surrounding soil. Also this record is affected by 1) a stress relief due to a larger hole size, 2) slip at the anchor soil interface during the impact, and 3) the radial jacking stress exerted by the anchor against the soil wall. These results 
together with the analytical calculations provide the necessary base for an appropriate interpretation to be made and used at production test sites.

The positioning of each of the sensors was selected such that a number of other test parameters could be evaluated. These include:

1) Casing effects

2) Jacking pressure effects

3) Assymmetry effects

4) P-wave effects and characteristics

5) Repeatability

6) Prior load history

Motion and force characteristics of the anchor system were also investigated with velocity transducers, accelerometers and a load cell. The velocity transducers and accelerometers were installed at several different points on the anchor while the load cell monitored the force applied by the hammer to the top of the anchor.

The combined results of this research effort together with a recommended simplified procedure for data interpretation, based on these results, are presented in the chapters that follow.

\section{3 SCOPE}

The report is organized into six chapters with appendices. Following this introductory section describing the extent of the work accomplished is Chapter 2 discussing the basic test equipment and procedures for obtaining and reducing production in situ test data. Emphasis is placed on describing the test and procedures, such that anyone interested in performing this test 
would know exactly how it is done.

An analytical description of the in situ impulse test is presented in Chapter 3. This chapter attempts to identify practical problems associated with the collection of field data together with theoretical problems, inherent to the wave generation and wave propagation phenomenon.

Chapter 4 is devoted to analytical studies of the test system. This work includes two finite element calculations performed to provide realistic "close in" velocity time histories for soils with known properties. With this computed data, various procedures used to estimate strain-dependent shear moduli could be applied to check the general validity of various data interpretation procedures.

The fifth chapter discusses the results of a special experimental test program conducted in an artificial sand fill. Emphasis of the tests, like Chapter 4, was to obtain "close in" results which could be evaluated to establish rational data interpretation procedures for processing production test information. This chapter evaluates these results.

The final chapter, Chapter 6, provides a recommended procedure for interpreting production data. This information is based on the results and studies presented in Chapters 4 and 5 . Also included is a brief discussion of planned future work on this particular project.

Seven appendices accompany the main text. Appendix A includes a description of the non-linear soil model used in the finite element calculations described in chapter 4. Appendix B presents typical experimental rosults obtained using the pneumatic anchor described in chapter 2. The soil properties, fill construction procedures and equipment installations at the Fort Lawton 
experimental test site are included in Appendices $C$ and $D$. These appendices describe much of the details of the soil conditions and test equipment installations not described in Chapter 5 .

Appendix E presents the results of a small literature review to establish sensor spacing requirements for the experimental test program. The final appendices, $F \& G$, are a comprehensive coverage of the special experimental tests and subsequent comparative and statistical studies conducted to evaluate the effects of various in situ test parameters on the in situ soil response measurements. This evaluation of the data was obtained as a major part of the special experimental program conducted at Fort Lawton and supplements that data provided in chapter 5. 
The development of the in situ impulse test involved 1) development of the basic test set up, 2) design and fabrication of test equipment, 3) development of field testing procedures and 4) development of data processing techniques. In previous studies, the test set-up was established through experimentation and bench testing in shallow borings with more simplified equipment. This testing was conducted to first check the feasibility of the procedure and in general establish boring spacing requirements, clarity of signature and the mechanics of the equipment. From these efforts, the impulse test as presently accomplished on a production basis is described in section 2.1.

Design and fabrication of test equipment also followed a line of progressive advancement in sophistication. In-hole equipment was improved and modified through testing to achieve optimum workability. Recording equipment originally consisting of strip chart recorders and oscilloscopes was updated by incorporating a magnetic tape recorder into a single console unit. This current equipment has greater resolution, greater recording flexibility, computer compatibility and greatly simplifies field recording procedures. The details of the test equipment are presented in Section 2.2 .

With reasonably well-established testing techniques and refined test equipment, current efforts have been concentrated on obtaining production data at different sites with different soil types. During this period six sites have been tested by this procedure to depths approaching 100 feet or greater. From this field testing, considerable experience has been gained. Based on this experience, section 2.3 describes in sequence the general field exploration equipment. and procedures used for exploring and testing these sites with this new test procedure. 
Finally the test data had to be processed in a consistent manner. Computer programs were developed for extracting the pertinent data points from each signature. The procedures for this are described in section 2.4 .

\subsection{TEST DESCRIPTION}

The in situ impulse test is similar in some respects to the cross-hole technique used in conventional geophysical explorations, in that it is based on the generation and recording of the propagation of shear waves through a mass of soil between two or more boreholes. A schematic of the test, shown in Fig. 1-1, includes a generating source and three or more recording stations arranged in a horizontal plane at a given depth, inside vertical boreholes. This arrangement may be radial as shown in the figure, or in an array in which the source and sensors are in a line. Borings containing free-field sensors may be either cased or uncased.

The primary difference between this test and other crosshole impulse-type tests is in the generation, magnitude and control of the shear waves. This test utilizes a controlled in-hole energy source in which shear waves dominate. The resultant recorded velocity-time signatures therefore have a distinct amplitude and shape as the shear pulse travels through the soil to successive recording stations. A consistent shape enables the identification of characteristic points on each pulse marking the time of passage of a wave through each recording station. The desired large amplitudes of the shear pulse are obtained by adjusting the spacing of the borings. For the general strains of interest $\left(10^{-3}\right.$ to $10^{-1}$ percent), sensors have generally been positioned in boreholes spaced about 4, 8 and 16 feet from the energy source. These are considerably closer spacings than used in the conventional cross-hole procedures. Thus the close spacing minimizes the inherent limitations 
in the cross-hole procedure of having waves reflecting or traveling over paths greatly different than assumed.

\subsection{TEST EQUIPMENT}

Major development efforts of testing equipment were accomplished in previous work and the results presented in previous progress reports (SW-AA, 1973\&1974). However, since this equipment has been gradually updated through minor improvements and modifications, the main features are summarized below.

\subsubsection{Anchor and Hammer Assembly}

'Two anchor and hammer assemblies have been developed to transmit the applied impact energy to the soil. The primary anchor used in the program to date consists of a heavy hydraulic system designed to operate in a 9 to 10 inch diameter borehole. This anchor is four feet long, weighs 195 pounds and is formed by three aluminum curved plate segments. The details of this anchor are shown in Fig. 2-la. The vertically oriented piston shown in this photograph is a 25 -ton capacity double acting hydraulic ram. This ram, when expanded, extends through a system of arms, forcing the curved plates outward and pressing them tightly against the walls of the borehole. A hand operated pump and gage is located at the ground surface to control and maintain the hydraulic pressure in this ram.

On top of the anchor plate segments as shown in Fig. 2-1b a striking plate, load cell, and two Belleville springs in series form the cushion. This cushion receives and transmits the impact of the hammer to the anchor assembly. The Bellevilie springs have a stiffness of about 300,000 lbs/in. The load cell is waterproofed, has a maximum capacity of $60 \mathrm{kips}$ and is designed to respond to high frequency motions. Auxiliary equipment with the load cell enables the load cell output to be 

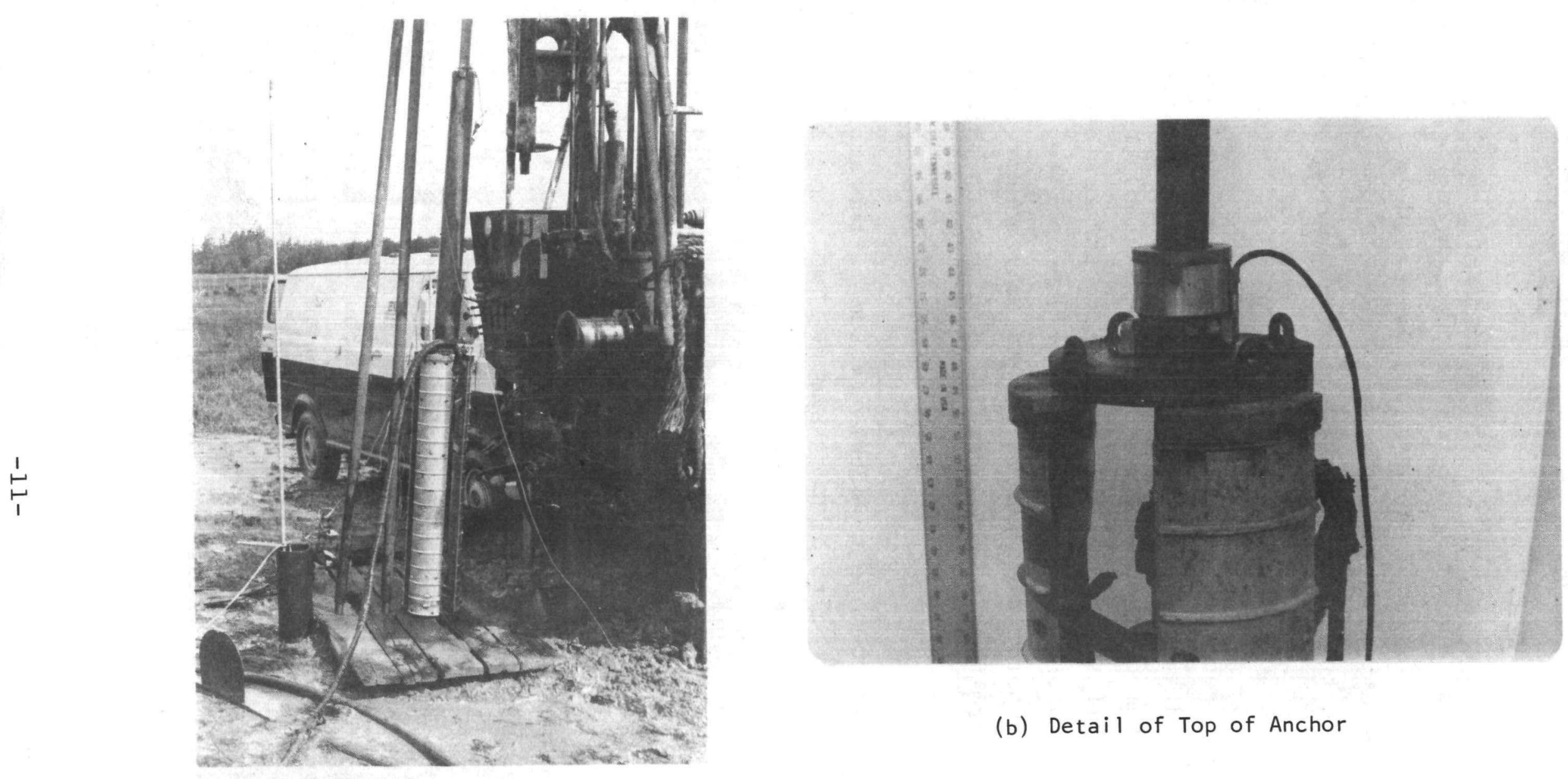

(b) Detail of Top of Anchor

(a) Anchor and Hammer Assembly

FIGURE 2-1 HYDRAULIC ANCHOR DETAILS 
recorded as a function of time simultaneously with the velocity measurements. Standard BW drill rod extends through the center of the springs, load cell, and striking plate and is fastened to the anchor by a yoke. This rod maintains alignment of the striking plate in the center of the anchor and also is used to raise and lower the entire assembly in the borehole.

During testing, the diameter of the expanded anchor can be determined at the ground surface by measuring, with a special key system on the bottom end of the BW rod, the stroke of the piston. If the hydraulic pressure in the ram and the diameter of the anchor are determined; by appropriate calibration, the radial horizontal stress at the soil/anchor interface also can be determined. Procedures for determining this value are described in Scction 3.1.3.

The last component in this system is the striking mass itself, herein referred to as the hammer. Two hammers were used and consisted of different size steel cylindrical weights, tapered slightly on the lower end. These weights are controlled from the ground surface by a thin steel hoisting cable. The hammers each have a 2-inch diameter hole through their center to be guided and centered in the hole by the BW drill rod. For production testing, 58 and 150 pound hammers were used. With these weights, a wide variation in energies was achieved by varying the height of arop.

The second anchor, tested to a lesser extent, was designed to operate in a 4-inch diameter borehole. This pneumatic anchor is five feet long, weighs about 150 pounds, and is formed by three steel plates surrounding an inflatable packer. The details of this anchor are shown in Fig. 2-2. The curved plates are made from double extra strong pipe cut into three curved segments. The inner core of this system consists of a high pressure packer unit (Burst pressure of 1300 psi). The 


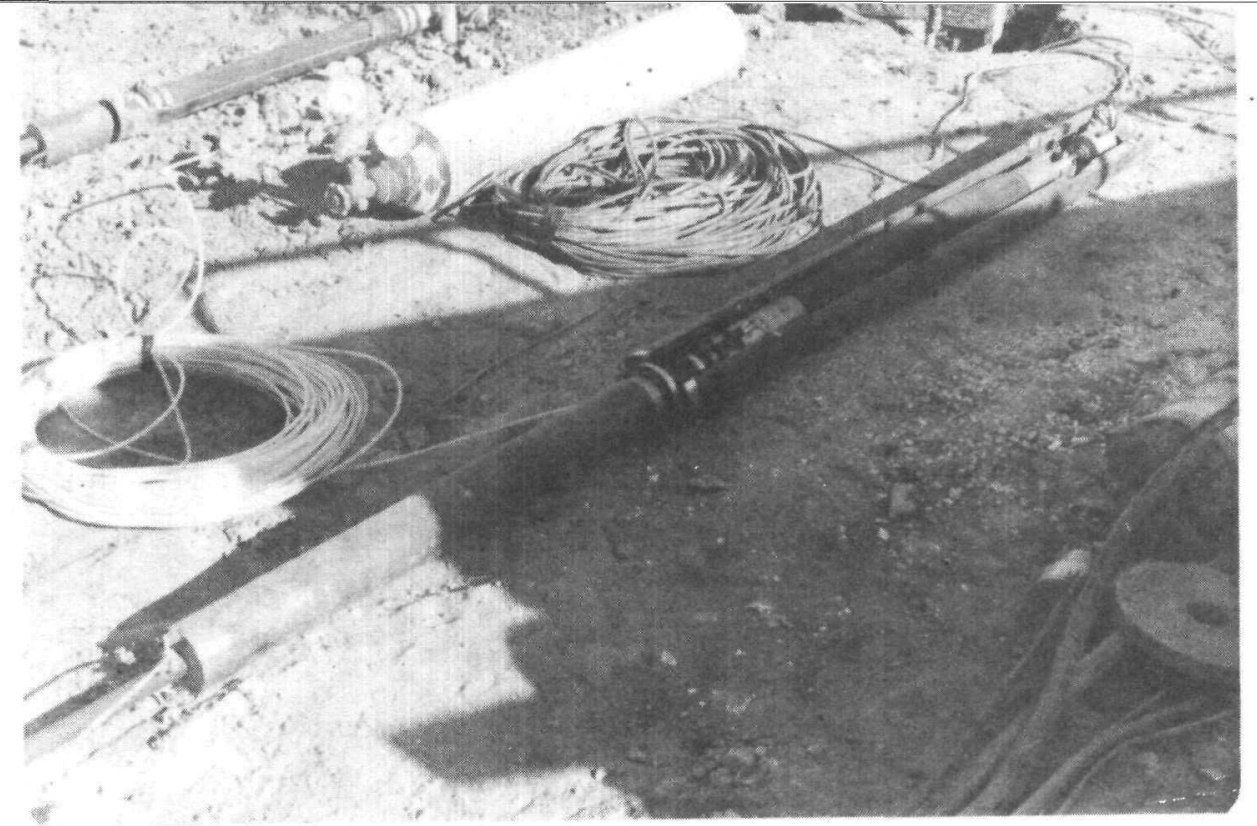

(a) Anchor and Hammer Assembly

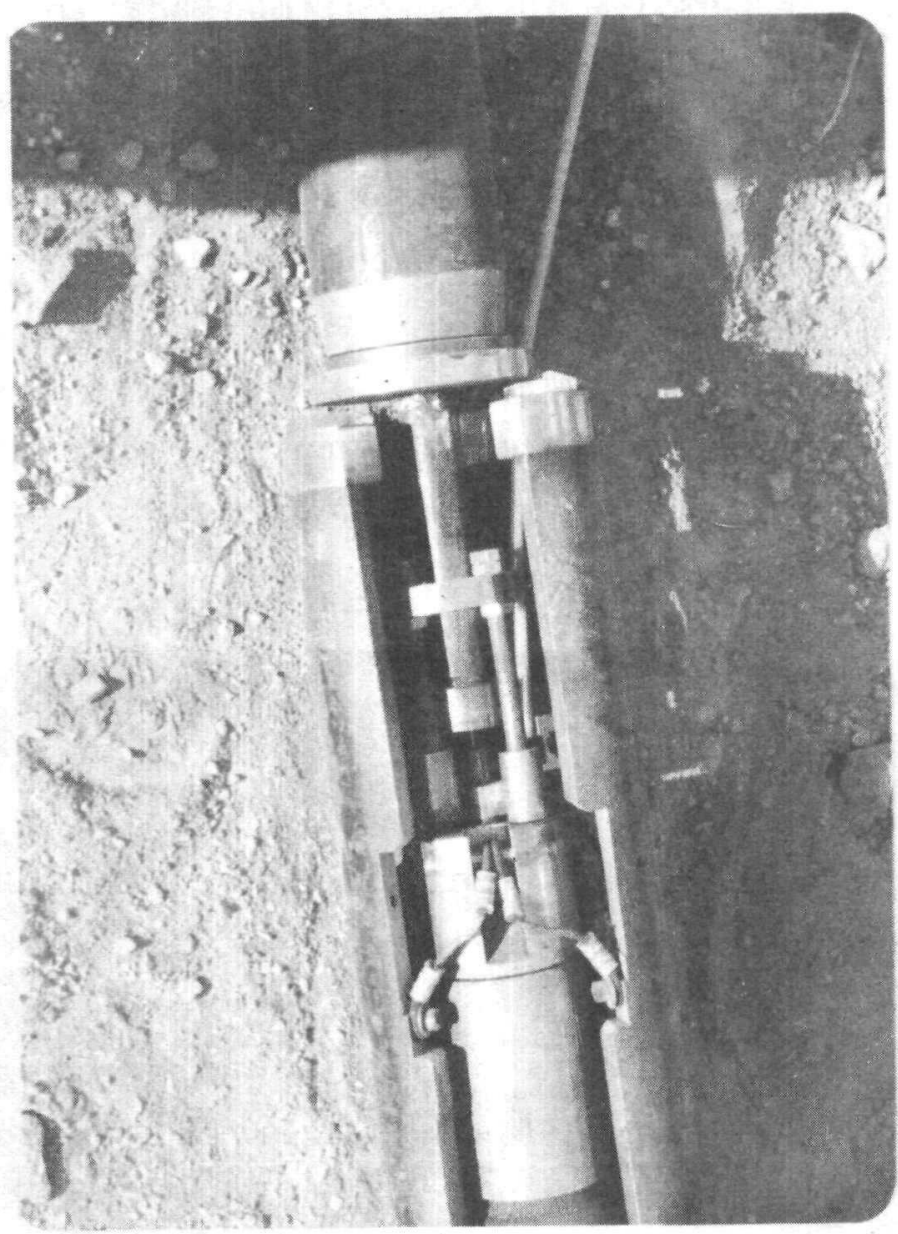

(b) Detail of Top of Anchor

FIGURE 2-2 PNEUMATIC ANCHOR DETAILS 


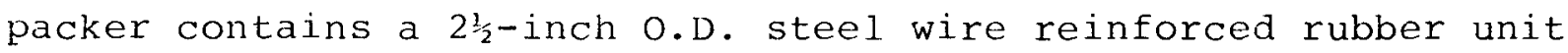
which expands up to about 6 inches in alameter under high air pressures. Coupling is achieved by expanding this unit outward pressing the plate segments tightly against the borehole walls. As the unit expands, the lower end of the packer contracts on an inner rod as a piston. This relieves the longitudinal stresses in the packer.

The entire anchor is held together by a system of eighteen short cables. When the anchor is expanded the cables compress or loosen causing the plates to hang in the borehole. Vertical alignment of the plates is maintained by this hanging action. When the air pressure is released the piston action of the packer pulls the system of cables together retracting the curved plates tightly against the inner packer.

The striking plate, springs, yoke and hammer systems shown in Fig. 2-2b, all operate on the same principal as the first anchor. The hammer for this system weighs about 90 pounds.

Both anchor and hammer assemblies are raised and lowered in the borehole by the drill rig used to advance the borings.

\subsection{Sensors}

The sensor units for this test consisted of vertically oriented velocity transducers (Mark Products L-10AK). These particular sensors have a natural frequency of 30 cycles per second. For these tests, each velocity transducer was placed in water-tight casings and fixed to a sensor holder. These holders consist of a strong, $1 \frac{1}{2}$-inch diameter inflatable, rubber, cylindrical balloon with two short aluminum heads fastened in each end. To make the holder act as a rigid element vertically, 
a thin solid steel rod extends through the rubber unit and is fixed to the two aluminum heads. The balloons are pressurized by compressed air and can be expanded to about 6 inches in diameter, if required, to achieve adequate coupling of the sensors with the walls of the boreholes. Orientation of the sensors was maintained at the ground surface through interconnecting 1/2-inch diameter, 10-foot long steel rods fixed to the aluminum head of the balloons. Details of the sensor and sensor holder are shown in Fig. 2-3.

\subsubsection{Recording Equipment}

The recording console used in the field to acquire velocity time histories from each sensor and load cell measurements is shown in Fig. 2-4. The console contains four basic units. The waveform recorder (Biomation Model 1015 Waveform Recorder) is the primary central data acquisition unit. This unit is capable of recording simultaneously and storing in memory, the velocity or load time histories independently from four sources. This stored data can be withdrawn from the recorder in either analog or digital form.

In analog form, the entire signal from each of the four channels is displayed simultaneously on the screen of the oscilloscope (lower right unit in Fig. 2-4). Because the information displayed on the oscilloscope is being taken from a memory bank, adjustments in time and amplitude of the signals are possible after the event. This feature enables small or critical data parts on any channel to be studied separately in detail or compared with critical parts on other channels. The oscilloscope was equipped with a camera mounted in front of the screen. Photographs, taken of the continuous display, provided not only a back up recording system but also valuable additional graphical records used for determining visually which tests were the best and should undergo further study and processing. 


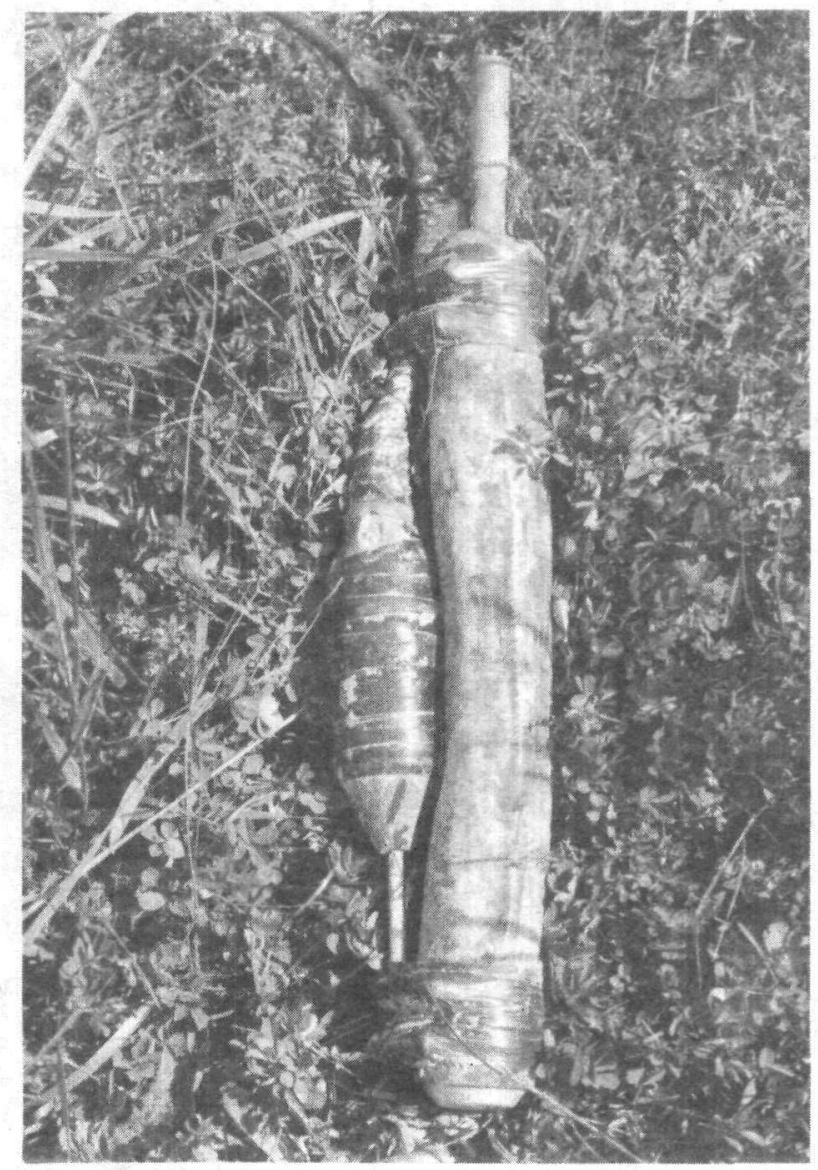

FIGURE 2-3 SENSOR AND SENSOR HOLDER 


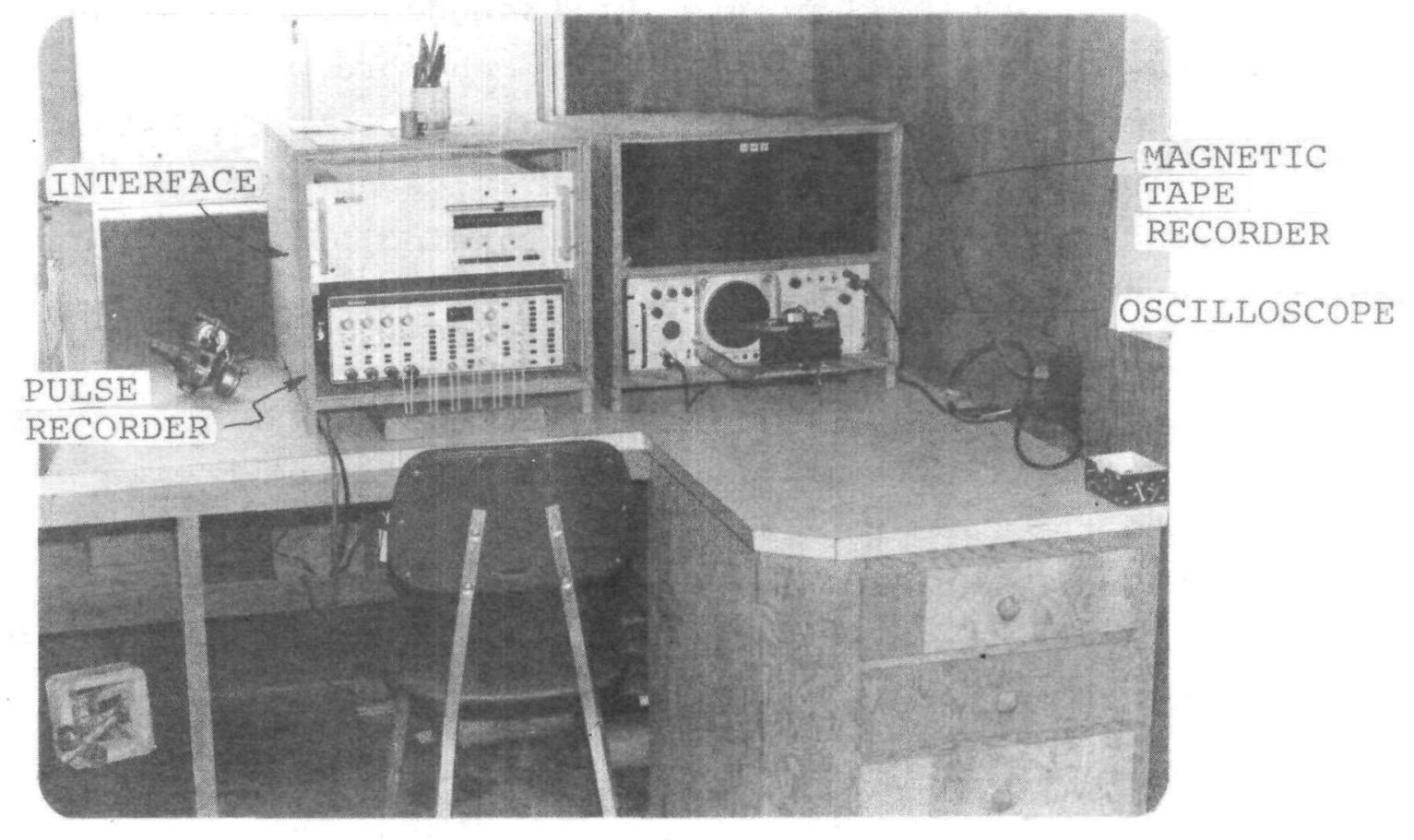

FIGURE 2-4 RECORDING EQUIPMENT 
In digital form, the data is withdrawn from the waveform recorder and placed directly on computer compatible digital magnetic tape. The top right unit in Fig. 2-4 is a Cipher Model 70M magnetic tape recorder. Records for each test can be digitally placed on the tape in about 30 seconds. Each of the four channels is printed in sequence as 1024 words or 4096 words in total. In most cases each word is printed with a time and amplitude resolution of $0.05 \mathrm{milliseconds}$ and one part in 1024, respectively. The recorder is capable of achieving a minimum and maximum time resolution ranging between $0.01 \mathrm{milli-}$ seconds and 10 seconds per word.

The interface unit provides the necessary electrical circuitry for transferring the digital data from the waveform recorder to the tape recorder. Also the interface unit contains manual control and automatic options which enable the test data, test labels, and both time and amplitude scales for each of the four channels to be organized systematically on the magnetic tape. This greatly simplifies data reduction, because the computer can read the necessary scaling information and test data on the tapes such that very little other data need be entered into the computer manually for complete processing.

\subsubsection{Surveying Equipment}

The precise horizontal distances between the velocity transducers in adjacent borings is determined with two electrical instruments, a spiral checking device and an inclinometer. These instruments are separately lowered into plastic casings either temporarily or permanently installed in each boring. Guide wheels on each instrument track in special continuous vertical slots grooved in the plastic casing. These slots assure accurate alignment and orientation of the instruments. The spiral checking device measures at five foot intervals any minor rotation of the grooves in the casing. By knowing the rotational 
position of the grooves at the ground surface, the change in rotation relative to the surface position can also be determined at any depth.

The inclinometer sensor also follows in the same slots and accurately determines the inclination of the sensor from the vertical. Inclinations are recorded on perpendicular planes at two foot intervals for the entire depth of the boring. With both inclination and rotational surveys combined, the horizontal displacements of the borehole at any depth relative to the top of the borehole can be established. By measuring the surface displacements with a tape, the distance (within an accuracy of about $0.1 \mathrm{ft}$ ) between respective holes at any depth can be computed.

\subsection{FIELD EXPLORATIONS AND TESTING PROCEDURES}

From past and current testing efforts at production sites, rather well established drilling and testing procedures have evolved. A small trailer and the necessary drilling equipment are first mobilized to each test site. The trailer transports the in situ equipment to each site and serves as a data acquisition center during field drilling and testing operations. Drilling rigs utilized to advance the borings and perform the in situ testing are conventional equipment and have consisted of either hollow stem auger rigs and/or rotary rigs.

The following paragraphs describe in sequence the general field exploration and testing program recommended for use in evaluating the variation in the in situ dynamic shear modulus with strain and depth at a potential nuclear power plant site. This work would generally supplement that normally performed on a routine basis. 
At each site four borings are drilled and tests performed to depths on the order of 100 to 200 feet. These borings are aligned in a straight line or in an array indicated in Fig. 1-1. The three sensor borings, spaced 4, 8 and 16 feet from the fourth anchor boring, are usually about 4 inches in diameter. The anchor boring is either 4 or 9 inches in diameter depending upon the anchor used.

Drilling is usually initiated by advancing and sampling one sensor boring for the entire (usually 200 feet) depth. This boring serves as an early base for defining the subsurface materials and assessing the likely performance of this hole to caving or deterioration. If caving is apparent, casing (plastic) should be installed in this as well as the remaining sensor borings. Otherwise testing can be accomplished in uncased holes. The anchor boring is usually drilled last, because it must be maintained and testing performed in an uncased hole.

As soon as each boring is completed three inch diameter casing is lowered into the hole through the drilling fluid for the full depth and rigidly fixed at the surface. The hole is then surveyed by lowering the two surveying probes inside the casing and recording the inclination and rotation characteristics of the casing. The casing is then removed from the hole shortly after the survey is completed. This entire operation usually takes about two to three hours per hole. If casing is required to prevent caving, the surveying operation of the sensor borings can be accomplished any time during the test program. 
Surveying requirements for the anchor boring depend upon the anchor and drilling equipment being used. If the 4-inch pneumatic anchor is used, the survey described above can be either accomplished before or at the completion of testing. If the larger 9-inch hole is required, it must be accomplished in one of two ways. If this hole is advanced by a continuous flight 3-3/8-inch I.D. hollow stem auger, the casing can be installed in the hollow stem. The hole can then be surveyed and the casing removed prior to pulling the auger.

If rotary procedures are used, a two-step operation is accomplished. First a pilot hole 4 inches in diameter is advanced for the full depth of the boring. This hole is then surveyed using the temporarily installed casing described previously. The pilot hole is then reamed to its full diameter. To prevent drift from the surveyed hole, the reaming bit should be aligned and guided with a pilot bit advancing at least five feet in front. Reaming can be accomplished in steps concurrent with testing or for the full depth. By drilling and testing in steps, borehole disturbances are minimized and the walls of the borehole are left exposed for shorter periods of time. This is an especially important consideration when caving or deterioration of the walls is a problem.

In advancing the four borings, care must be taken to assure reasonable verticality of the holes especially the two nearest the anchor. Excessive drift in the alignment can result in holes converging to a common distance from the anchor. This, in effect, causes two potential data stations to act as one. This may require the drilling of extra holes. If initial care is taken to properly align the drilling rig and long heavy drill collars are used in the drilling operation, this problem is minimized. 
Once all holes have been completed and surveyed, in situ testing is conducted usually at 5-foot intervals. Testing usually starts at the top of the boreholes and progresses downward.

The anchor and hammer assembly is raised and lowered on BW drill rod to the prescribed test depth by the drilling rig. The rig is needed for this operation because it is capable of 1) flushing the hole prior to the start of testing, 2) pushing the anchor assembly through bridges or tight spots, 3) pulling the anchor assembly loose from the borehole walls following testing, and 4) pumping water or drilling fluid through the center of the assembly to wash out soil particles that have collected during the testing operation.

Coupling of the anchor is accomplished by gradually increasing the hydraulic pressure in the 25 ton ram until a stable value is reached. To monitor the coupling of this anchor assembly, measurements of jacking pressure and the vertical stroke of the ram (using the special key system) are checked periodically during the jacking process and during the test itself. With appropriate calibration, these two values enable the diameter of the anchor and the resulting radial stress being applied to be monitored. As will be indicated in section 3.1.3 and Appendix G, achieving optimum coupling is very important. Either too high or too low a pressure can affect the strains achieved. The number of strokes applied to the hydraulic pump and the general resistance to pumping provide another satisfactory index of the increasing soil resistance to jacking forces.

For the pneumatic anchor, air pressure is used to expand the anchor assembly and couple it to the sides of the borehole wall. Initial coupling of this system is checked by pulling slightly on the rods with the drill rig. Immediate resistance 
will indicate coupling.

Free-field sensors and their holders are lightweight (less than 3 pounds) and can be raised and lowered usually with interconnecting 1/2-inch diameter, 10-foot long, thin steel rods. The pin connecting ends of the rods are designed such that proper orientation or positioning of the sensors within the borehole could be quickly and easily established.

Coupling of each sensor is achieved by inflating the rubber packer holder with compressed nitrogen. Firm coupling is verified by pulling or pushing manually on the interconnecting rods. Also to assure continued coupling during testing, the rods are left free standing above the ground surface. Thus their weight is supported entirely by the sensor holder providing a simple monitor of the coupling effectiveness. If the coupling becomes loose, the rods would drop several inches and catch a horizontal clamp on the top of surface casing.

For each depth, a trial impact is first applied. From this impact, velocity time histories are recorded from four vertically oriented sensors, (i.e., where shear waves were the dominant particle motion). These records are displayed continuously on the screen of the oscilloscope. This display is then inspected by the field engineer to determine if all equipment is operating properly (i.e., proper coupling of both anchor and sensors, clear impact, and proper scales on recording equipment). The impact is then repeated and other records obtained the same way until four clear properly scaled time history signatures are obtained from one impact. Two or three tentative trials is usually sufficient to obtain a good test. This last test is made a permanent record by first taking photographs of the display on the oscilloscope. Because portions of each signature may be expanded or the scale changed after the event, more than one photograph is often justified. The information is then recorded 
on a basic data sheet and in diyital form on a computer compatible magnetic tape recorder for the later study and evaluation. The photographs enable the overall signatures to be studied and assessed qualitatively while the digital records provide the necessary time and amplitude resolution needed in this test.

At a given test depth, about six tests are taken; usually three tests at two different energy levels. The different energy levels are achieved by varying the height of drop of the one of the two hammers. For most soils, the drop commonly varies between six inches and two feet. In addition to measurements of vertical particle velocities, measurements of load applied to the anchor and horizontal particle velocities from free field sensors are also recorded. The load provides for partial interpretation of the shear wave velocity at the anchor and the horizontal velocity measurements enable the P-wave velocity and the water table to be determined.

\section{4 DATA PROCESSING}

In order to determine the shear modulus, G, (or shear wave velocity, $\mathrm{V}_{\mathrm{s}}$ ) as a function of strain, four basic measurements are obtained in the performance of the test as listed below:

1) the distances between the anchor and the free field sensors

2) the arrival time at each sensor

3) the peak particle velocity at each sensor

4) the time of first impact of the hammer to the anchor

In addition, to study the response of the anchor, the peak force amplitude recorded on the load cell, as well as various time points that define the shape of the recorded load impulse have been processed. 
The distance between borings at corresponding depths is determined by precise field surveys of each hole. The remaining parameters are obtained from time history measurements using a load cell and velocity transducers positioned on and within the anchor respectively, and vertically-oriented velocity transducers located in adjacent borings. A sample of the particle velocity versus time records for a test is shown in Fig. 2-5. This photograph shows the analog signals as displayed on the screen of an oscilloscope. The data actually processed and reduced are in digital form on magnetic tape. In general, data processing for production tests can be divided into three phases: 1) initial checkout of the data, 2) computer processing of the response measurements, and 3) final determination of the velocity (or modulus) strain relationships.

\subsubsection{Initial Checkout Phase}

The initial step in this phase, upon receipt of the magnetic tapes, oscilloscope photographs, and summary data sheets, was to copy the data from the field tapes onto separate magnetic tapes. The purpose of this step was threefold. First, the computer facilities were able to transcribe the data onto a separate tape at a much higher voltage level than was possible from the field recorder. This greatly facilitated subsequent data processing operations. Second, the original field tapes provided backup data storage, in the event the tapes generated were damaged. Finally, any portions of the field tapes that contained unrecoverable data could be quickly identified. If these portions were small, they were skipped so that the remainder of the tapes could be processed. In the unlikely event that a major portion of the data was unrecoverable, the engineers could be notified while still in the field, and the measurements corresponding to the unrecoverable data could be repeated. 


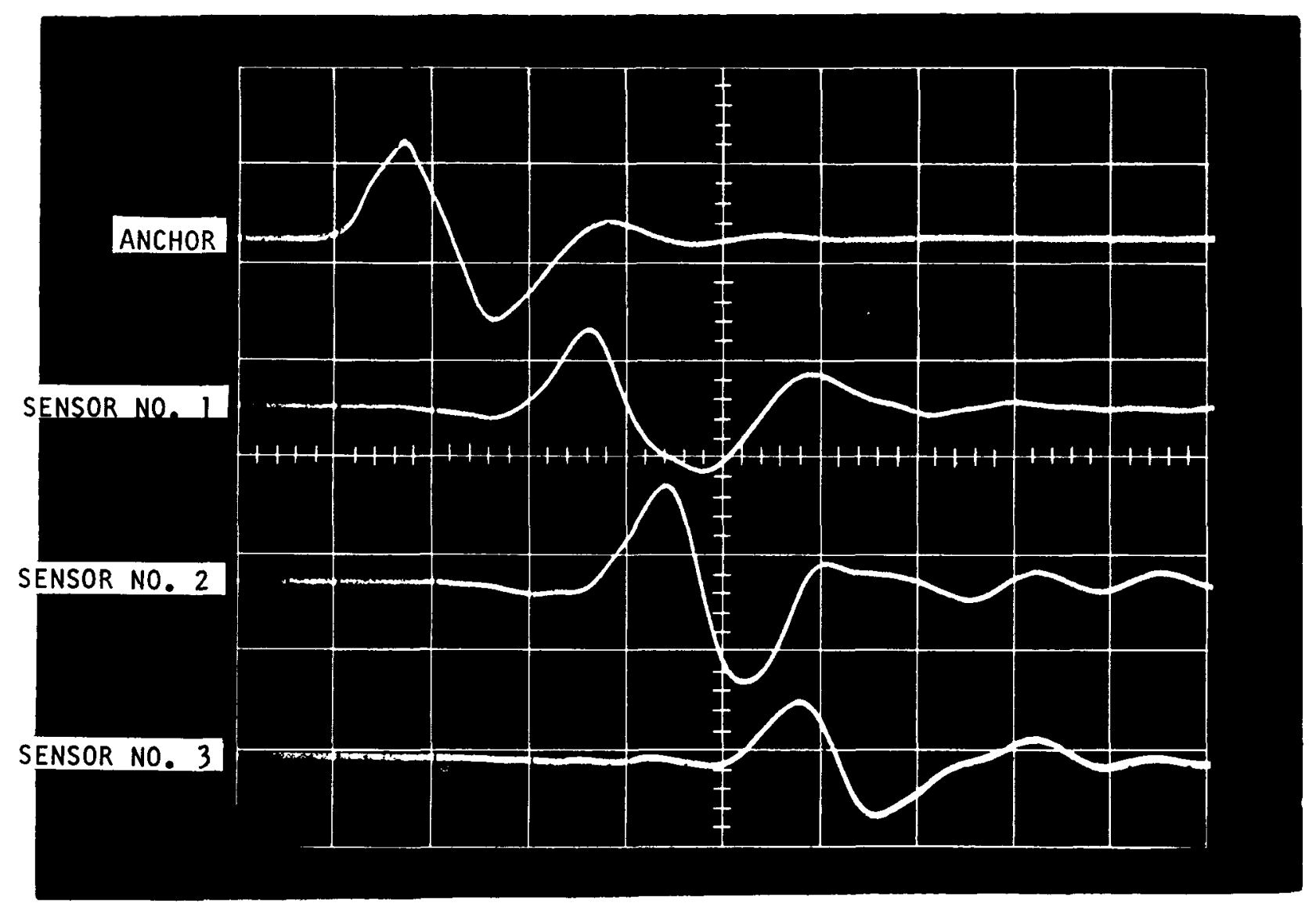

FIGURE 2-5 TYPICAL VELOCITY TIME SIGNATURES DISPLAYED ON THE SCREEN OF THE OSCILLOSCOPE 
Once the tapes were copied, further processing of the data was initiated. The copied tapes were dumped and the listings were checked for bad tape records and improper data or labels. Then the following data on the test summary sheets were compared with the tape dumps, where appropriate, and were used as input to the processing program described in the next section.

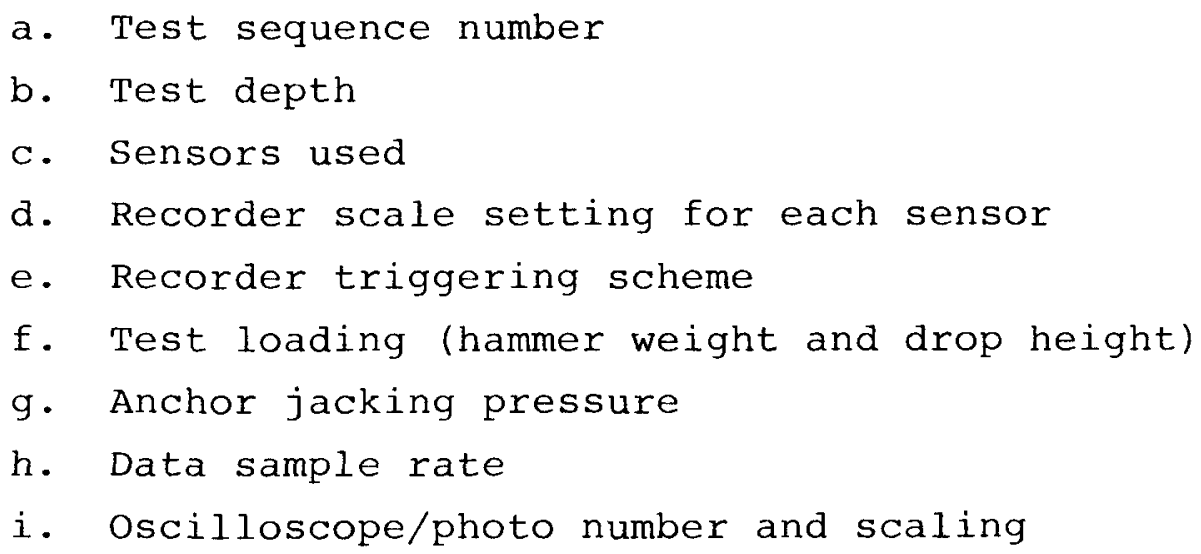

The above comparison served to indicate whether any changes would be required to the data processing programs and also provided a basis for noting in advance those tests that were duplicates or missing and those that did not require subsequent processing. Only vertical velocity records and load cell measurements were processed according to the procedures described herein. Horizontal time of arrival measurements for determining $\mathrm{P}$-wave velocities were determined manually from the initial tape listings.

\subsubsection{Computer Processing Phase}

Once the initial checkout phase was completed, the data were then read into a specially developed computer program which processed the data according to the following steps:
a. Reformat data
b. Scale data into engineering units 
c. Label tests and test channels

d. Detect peaks and zero crossings

e. Compute time delays

f. Store and manage results for easy reaccess

g. Print results in suitable report format

The first step in this final processing (Step a) involved the proper reading and decoding of the binary coded decimal formatted data into four digit integers in the digitizer range of 0 to 1024 counts. Then, in step $b$, the 12-character test label preceding each test was decoded into six 2-character parameters which represented the test sequence number, the sampling interval and the recorder scale setting for each of the four data channels recorded in each test. In addition, the computer program was designed to automatically decode the label for scaling purposes and also corrected for zero-amplitude offsets in the data.* The recorder scale setting was combined with the transducer sensitivity factor to produce a resultant scale factor. The zero-amplitude offset level was subtracted from the data prior to scaling.

Step $c$ of the final processing involved labeling the test channels with appropriate sensor names, test numbers, and test sites. This data was stored for future identification of the data processed in subsequent steps.

The Recording system used in the field identified only positive integers ( 0 to 1024 counts) when recording the velocity histories. Therefore, the zero level of the velocity history was assigned a positive integer value by the Biomation Recorder; this integer value had to be sufficiently large so that the largest-amplitude negative velocities could be recorded using a positive integer. In processing the data, corrections for this zero-amplitude offset had to be made; in providing these corrections, the first data value. from each channel was assumed to correspond to this offset level. 
In step d, key amplitude and time points of the velocity and load cell data were identified. These points corresponded to those considered significant for subsequent assessment of the velocity-strain relationships (Fig. 2-6). The times of arrival at adjacent sensors of the first downward velocity peak and the first crossing ("TPOS PK" and "TZEROX" respectively in Fig. 2-6a) were then used to compute time delays in step e. Further discussion and evaluation of various methods for computing shear velocities is presented in Chapters 4,5 and 6.

The final steps in this phase (Steps $f$ and $g$ ) consisted of storage of the processed data so that it could be easily retrieved and, in addition, a printout of the summary information developed in Steps $d$ and e. A sample of the pertinent data extracted from these tapes is summarized in Fig. 2-7. For each test at each depth, this printout included the energy level, jacking pressure, test number, the time and amplitude measurements identified in Fig. 2-6, and the various time delays between adjacent sensors.

\subsubsection{Determination of Velocity-Strain Relationships}

Having determined the above parameters from the data tapes, the time data are next plotted with distance as shown in Fig. 2-8. As discussed in chapter 6, the time of first crossing (TZEROX) is most commonly used to identify the arrival time in the free field sensors. This time point from each sensor record, together with the measured position of each sensor relative to the anchor, was used to construct the position versus time curve shown in Fig. 2-8. The slope of this curve at each sensor was used to estimate the shear wave velocity at that location. Since the wave decays rapidly in the nearanchor regions, the soil strains likewise attenuate with increasing distance from the energy source. The strains in the free field are then computed assuming the wave travels as a 


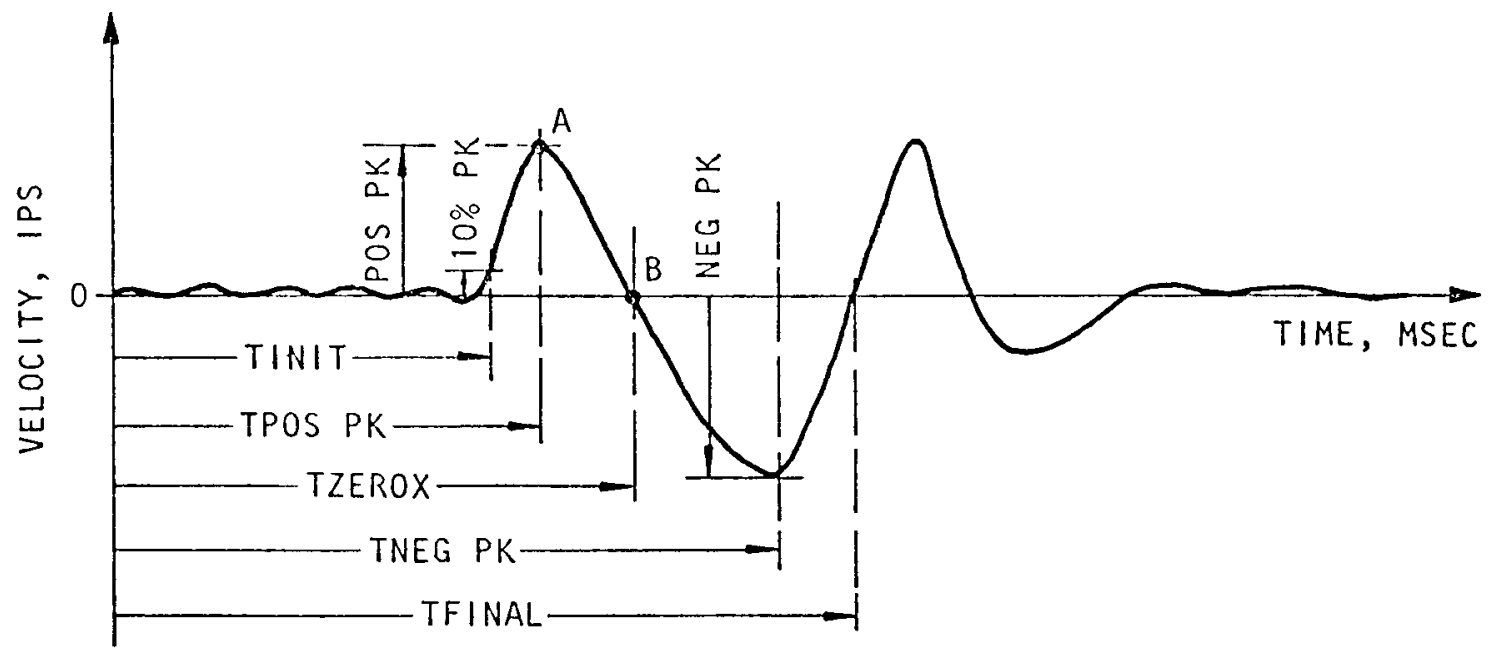

TIME DELAYS: POSITIVE PEAK -- BASED ON ARRIVAL TIME OF POINT A ZERO CROSSING -- BASED ON ARRIVAL TIME OF POINT B

(a) Vertical velocity measurements

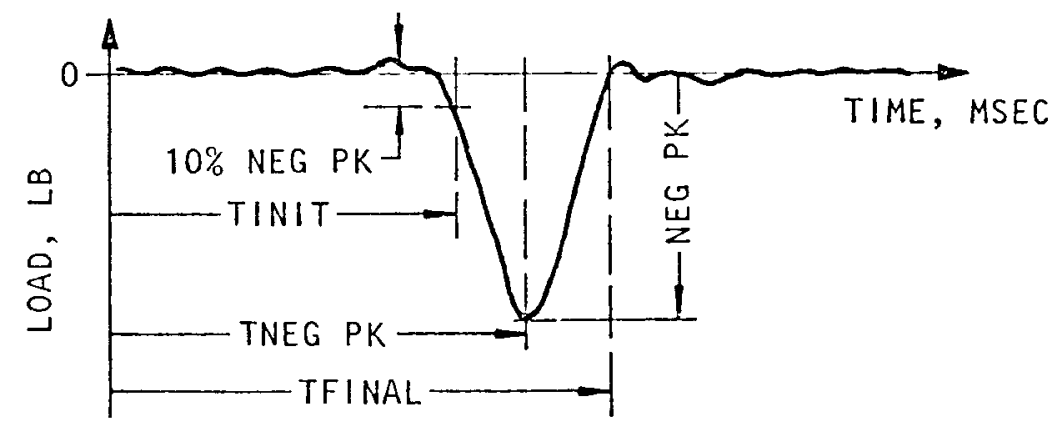

NOTE (FOR BOTH VERTICAL VELOCITY AND LOAD CELL MEASUREIIEIJTS):

ZERO TIME REPRESENTS FIRST RECORDED DATA VALUE

ZERO AMPLITUDE BASED ON BIAS OF FIRST RECORDED DATA

(b) Load cell measurements

FIGURE 2-6 DATA REQUIRED FROM PRODUCTION TESTS 
PRUDUCTION TESTS EL CENTRD SITE

DEPTH $\otimes$ FEET

(TIME IN MSEC, VELOCITY IN IPS, LOAD IN LH)

TESTS 27 AND 28 (TAPE $(-3)$

ENERGY LEVEL $=150$ LB $\times 2,0$ IN JACKING PRESSURE $=1500$ PS!

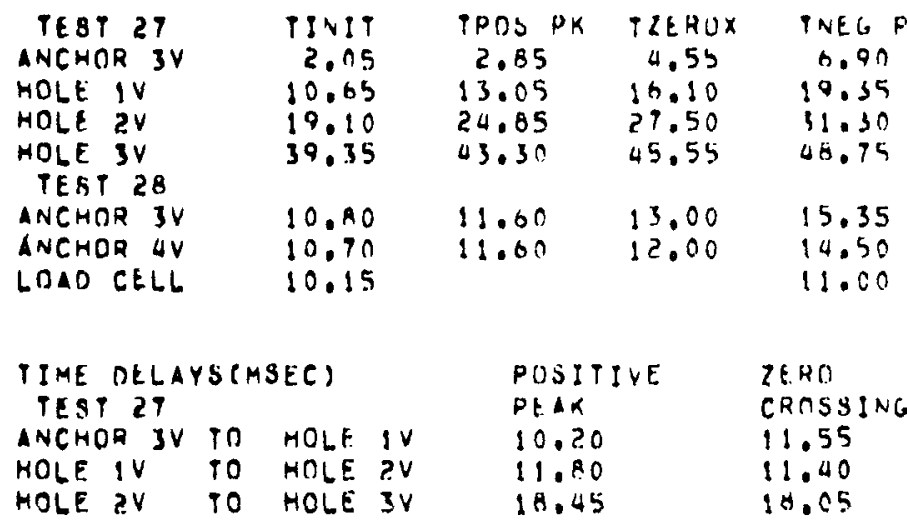

TESTS 30 ANO 31 (TAPE COS)

ENERGY LEVEL 150 LB $\times 1.0 \mathrm{FT}$ JACKING PRESSURE \& 1500 PS:

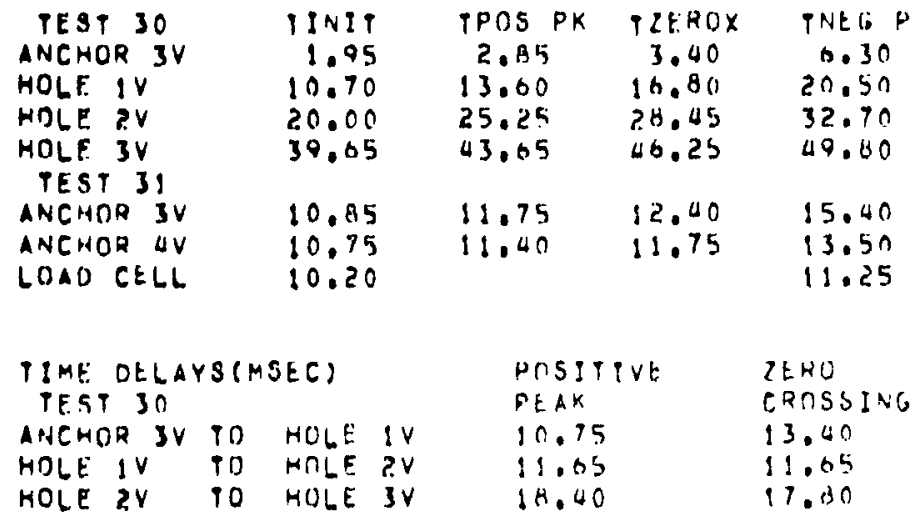

FIGURE 2-7 SAMPLE OUTPUT FROM PRODUCTION TESTS 


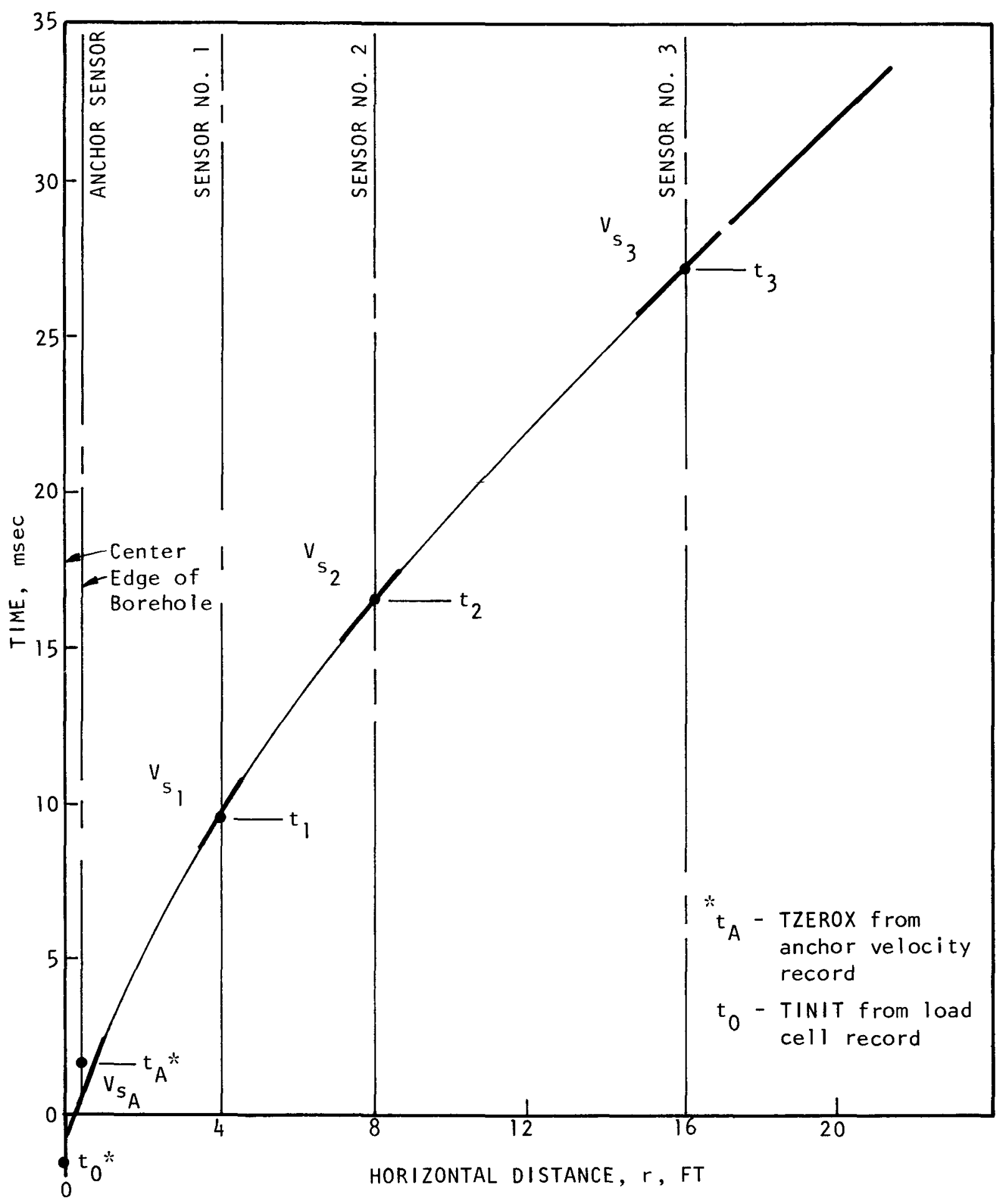

FIGURE 2-8 TIME OF ARRIVAL AT PEAK STRESS VS DISTANCE FROM ANCHOR 
plane wave (1.e., $\left.\gamma=\frac{V_{p}}{V_{s}}\right)$. The procedures for determination of shear-wave velocity ${ }^{S}$ and strains at the anchor are not as stralghtforward. A fuller treatment of the meaning and construction procedures of individual points at the anchor, based on the results of this special study are presented in Chapters 5 and 6 .

Having determined the shear wave velocity and corresponding shear strain for each station, the shear modulus is computed by:

$$
\mathrm{G}=\rho \mathrm{V}_{\mathrm{s}}^{2}
$$

where $\rho$ is the mass density of the soll.

Both $G$ and $V_{S}$ can then be plotted with strain as shown in Fig. 2-9. This procedure 15 repeated for each test depth, from which profiles of shear modulus at specific strain levels can be estimated (F1g. 2-10). These profiles can be used to represent soll material properties for use as input to site response studies. 


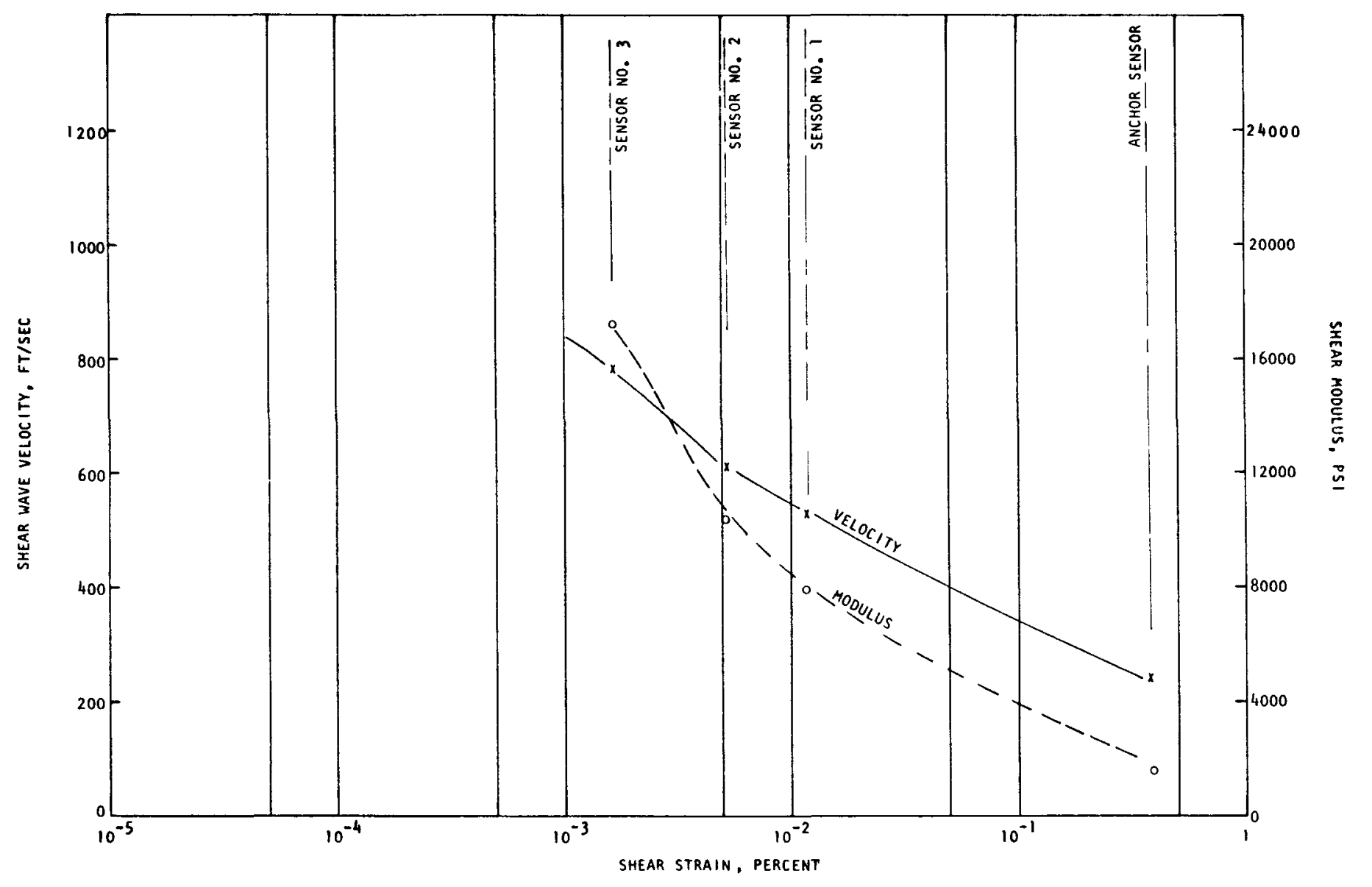

FIGURE 2-9 EXAMPLE PLOT OF MODULUS AND VELOCITY VS STRAIN 


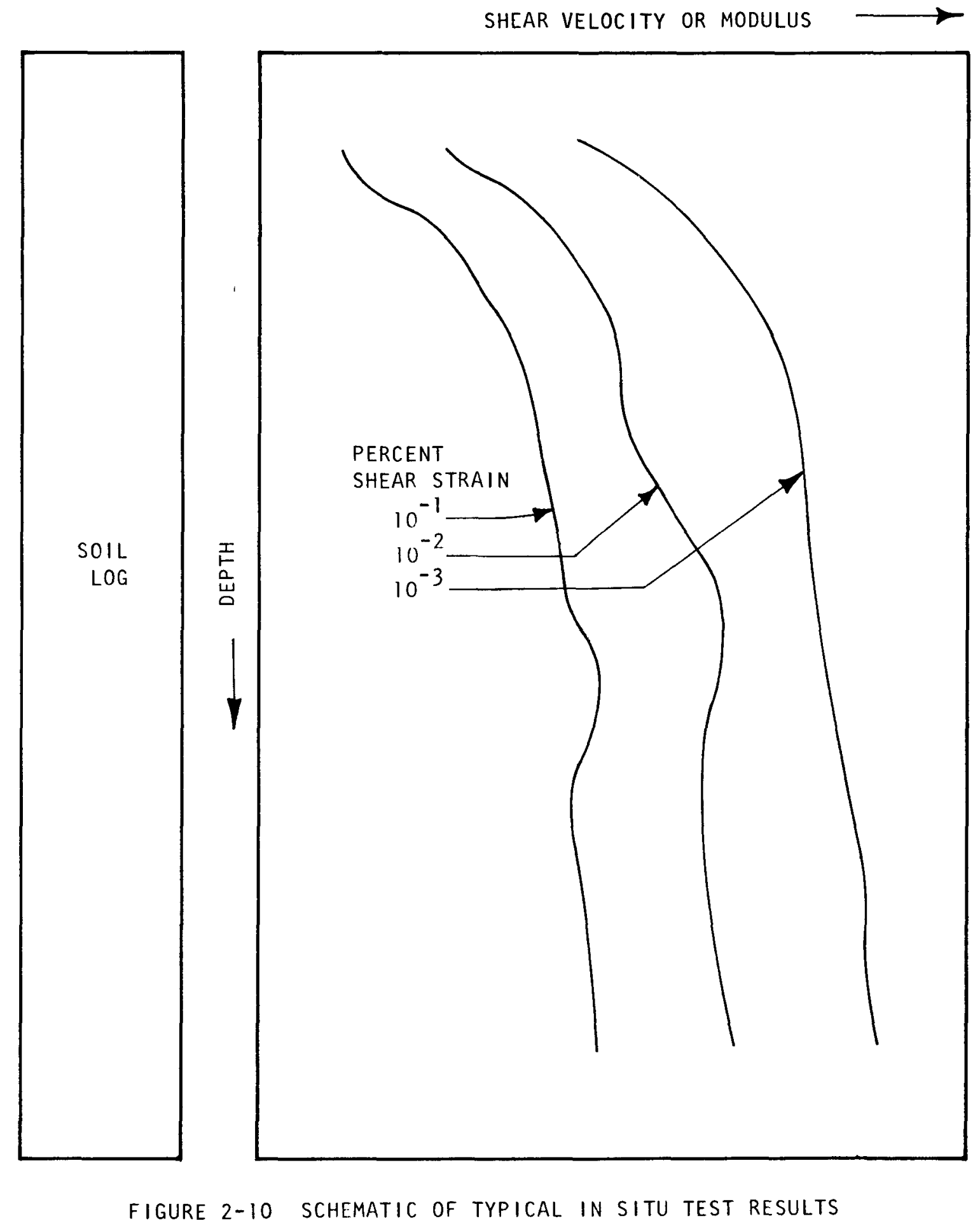

$-35-$ 


\section{ANALYTICAL DESCRIPTION OF THE \\ IN SITU IMPULSE TEST}

The purpose of this chapter is to present an analytical discussion of the wave propagation phenomena and the test conditions of the in situ test procedure. To carry this out, the chapter is divided into three sections. Section 3.1 considers potential effects of such factors as arilling of the boreholes and installation of the anchor and sensors on the in situ soil properties. The dynamics of the coupled anchor-soil system are briefly discussed in section 3.2, while section 3.3 develops those particular fundamentals of basic wave theory that are associated with the in situ test approach.

\subsection{ANALYSIS OF TEST CONDITIONS}

Three conditions exist during the drilling and testing operation which may cause changes within the soil mass being tested. These are 1) stress relief around borings, 2) disturbance caused by advancing borings through the medium and 3) horizontal jacking stresses applied in coupling the anchor to the borehole wall. The following sections discuss the likely qualitative effect of each condition.

\subsubsection{Conditions Caused by Stress Relief}

The change in the state of stress caused by the removal of the soil in a drill hole has been studied by Terzaghi, 1943 and Westergaard, 1940. As a result of stress relief, a zone of plastic equilibrium develops around the opening. For a soil whose shearing strength is given by:

$$
S=\mathbf{C}+\bar{\sigma} \tan \phi
$$


where: c is the cohesion, $\vec{\sigma}$ the effective normal stress on the plane of shear, and $\phi$ the angle of internal friction, the boundary between the plastic zone and the surrounding elastic zone, is located at a radius $r_{e}$, from the center of the hole. This distance is given by the following equation (Terzaghi, 1943).

$$
r_{e}=r_{0}\left\{\frac{2\left[\left(N_{\phi}-1\right) \gamma z+2 c \sqrt{N_{\phi}}:\right.}{\left(N_{\phi}+1\right)\left[\left(N_{\phi}-1\right) \sigma_{r o}+2 c \sqrt{N_{\phi}} ?\right.}\right\} \overline{\left(N_{\phi}-\overline{1}\right)}
$$

where: $r_{0}$ is the radius of the hole

$$
\begin{aligned}
& \sigma_{\text {ro }} \text { is the normal stress applied at the walls } \\
& \text { of the hole at a depth } z \\
& \gamma \text { is the unit weight of the soil } \\
& \mathrm{N}_{\phi}=\tan ^{2}\left(45^{\circ}+\frac{\phi}{2}\right)
\end{aligned}
$$

From this equation, if no supporting normal stress is provided, $\left(\sigma_{\text {ro }}=0\right)$, failure will occur at any depth for a cohesionless soil. For a clay with $\phi=0$, the equation indicates that no plastic zone will develop for depths $z$ smaller than:

$$
z=\frac{C}{\gamma}
$$

In the normal drilling procedures for the in situ test, drilling mud is used to remove drill cutting, to counterbalance the hydrostatic head of groundwater, and to create a "mud jacket" around the walls. These last two features produce a counteracting normal stress, $\sigma_{\text {ro' }}$ which minimizes the plastic zone created. The actual extent of the plastic zone depends on the ratio between the free field stress and the applied supporting stress, (oro), together with the shear strength of the soil, at each depth.

To illustrate the importance of the thickness of the 
plastic zone, consider a sand deposit with $\mathrm{c}=0, \phi=30^{\circ}, \mathrm{N}_{\phi}=3$. Equation 3.2 becomes:

$$
\frac{r_{\mathrm{e}}}{r_{\mathrm{o}}}=\left(\frac{\gamma z}{2 \sigma_{\mathrm{ro}}}\right)^{\frac{1}{2}}
$$

and the following values are computed:

Table 3.1

Extent of Plastic Zone for Sand, $\phi=30^{\circ}$

$\frac{{ }^{\sigma} r_{0}}{r_{z}} \quad \frac{r_{e}}{r_{0}} \quad$ Result

$\begin{array}{lcl}1 & .71 & \text { No plastic zone developed } \\ .5 & 1 & \text { No plastic zone developed } \\ .25 & 1.41 & \text { Plastic zone, thickness }=.41 \mathrm{r}_{0} \\ .1 & 2.24 & \text { Plastic zone, thickness }=1.24 \mathrm{r}_{0} \\ .05 & 3.16 & \text { Plastic zone, thickness }=2.16 \mathrm{r}_{0}\end{array}$

Determination of the effective supporting stresses of drilling muds is difficult. However, local sloughing of borehole walls is an indication that the plastic zone is enlarging. To maintain the drill hole, use of increasingly heavier muds is required. This increase in mud thickness, in effect, automatically increases this supporting stress keeping the plastic zone to a minimum. As a rough estimate the ratio of ${ }^{\sigma} \mathrm{ro} / \mathrm{rz}$ for a reasonably thick mud is probably of the order of .1 to .25 . Based on this, and realizing that even if caving does not occur; for average test depths o: 100 to 200 feet, a plastic zone affecting a concentric area around a 9 to lo-inch anchor borehole and 4-inch sensor borchole is probably about one-half to 
one foot and several inches thick, respectively. This zone is considered negligible for the smaller holes. For the large anchor, this zone if maintained, by using heavy drilling muds should produce only a minor difference in measured data. The difference is probably well within the accuracy of other test measurements (especially those made at the anchor).

\subsubsection{Disturbances Caused by Drilling}

Drilling operations produce disturbances of the soils around a borehole. The degree of disturbance depends on the size of the hole, the properties of the materials, the equipment and procedures, and the experience of the drillers. Published information relative to the degree of disturbance of the walls of boreholes is difficult to find.

Using rotary methods or the hollow-stem auger excessive disturbances can occur in about four ways. Excessive water pressures and turbulent flow of the drilling mud, especially at the bottom of the borehole, can locally erode or deteriorate surrounding soils rapidly. Rapid withdrawal or lowering of drilling tools can gouge and smear walls. The rotation of drilling tools at one depth for excessive periods can cause enlarged zones. Finally use of flexible tools and no drill collar can cause not only crooked holes but also irregular cylinarical shapes with possible protrusions, cavities and local discontinuities. In all cases, these effects of disturbances of the soil especially for the larger anchor hole are important field considerations for the field inspector and driller performing the test and can be minimized with due care. For the soils tested, the zone of excessive disturbance caused by drilling operations is estimated to be confined probably to a thicknness of one to two inches. 
The most important operation of the installation of the wave generating station is coupling of the anchor plates with the soil mass. In this operation, both the effectiveness of coupling and the radial jacking stresses must be determined. The effectiveness of coupling is a field consideration and requires close tolerances in control of the shape and diameter of the borehole. If the hole size is maintained (i.e., is less than 11 inches for the hydraulic anchor and 6 inches for the pneumatic anchor), the lateral travel of the equipment components is usually sufficient to press through the mud jacket and the concentric layer of soils disturbed by drilling and firmly grip the undisturbed soils.

Further gripping power is achieved at the interface of the large diameter anchor through evenly spaced horizontal wedges protruding from the contact surface of the anchor plates. These wedges, spaced vertically every three inches, have a triangular shape $1 / 4$ inch at the base by $1 / 4$ inch high. These wedges, when pressed into the soil increase the friction coefficient from one corresponding to smooth contact between aluminum and soil to that of soil to soil. Also the wedges provide some confinement to limit the spreading of local bearing failure zones which may result from stress concentrations caused by minor irregularities in the shape of the hole.

Proper selection of an optimum radial jacking stress is also a field consideration in establishing the effectiveness of coupling. The optimum value should be large enough to minimize slippage and thus avoid the creation of distorted signatures due to this slippage. On the other hand, this stress should not exceed a limit value as discussed in Appendix G. If this jacking stress combined with the stresses from the impact exceeds the bearing capacity of the soil, local shear failure results. This 
condition, while not distorting recorded signal shapes, reduces greatly the energy transmitted to the soil and thus the strains achieved in the free field. By experimental testing at the first few test depths in a production test program, this optimum stress can be readily estimated.

For close control of coupling in the field, the numerical values of radial jacking stress on the anchor plates can be determined and is required for later use in data interpretation procedures. As discussed earlier (Section 2.2.1) measurement of the stroke of the hydraulic ram made from the ground surface, and the jack pressure enable the determination of both the radial stress and the diameter of the anchor during testing.

The radial stress is determined by the equation:

$$
\sigma_{r}=\frac{2}{3} \sigma_{j} \frac{\Omega}{\AA_{L}} \cot \alpha
$$

where: $\sigma_{r}$ radial stress

$\sigma_{j}$ jack or ram pressure

$\Omega$ effective area of the piston of the hydraulic ram

${ }^{A} L$ lateral area of one anchor plate

$(x$ angle of the arms of the anchor with the horizontal plane

The above symbols in this equation are also identified in Fig. 3-1. The diameter of the anchor is determined by the following equation.

$$
D=a+2 a \sqrt{1-\left(\frac{L-[(1-g)-t]}{2 a}\right)}^{2}
$$




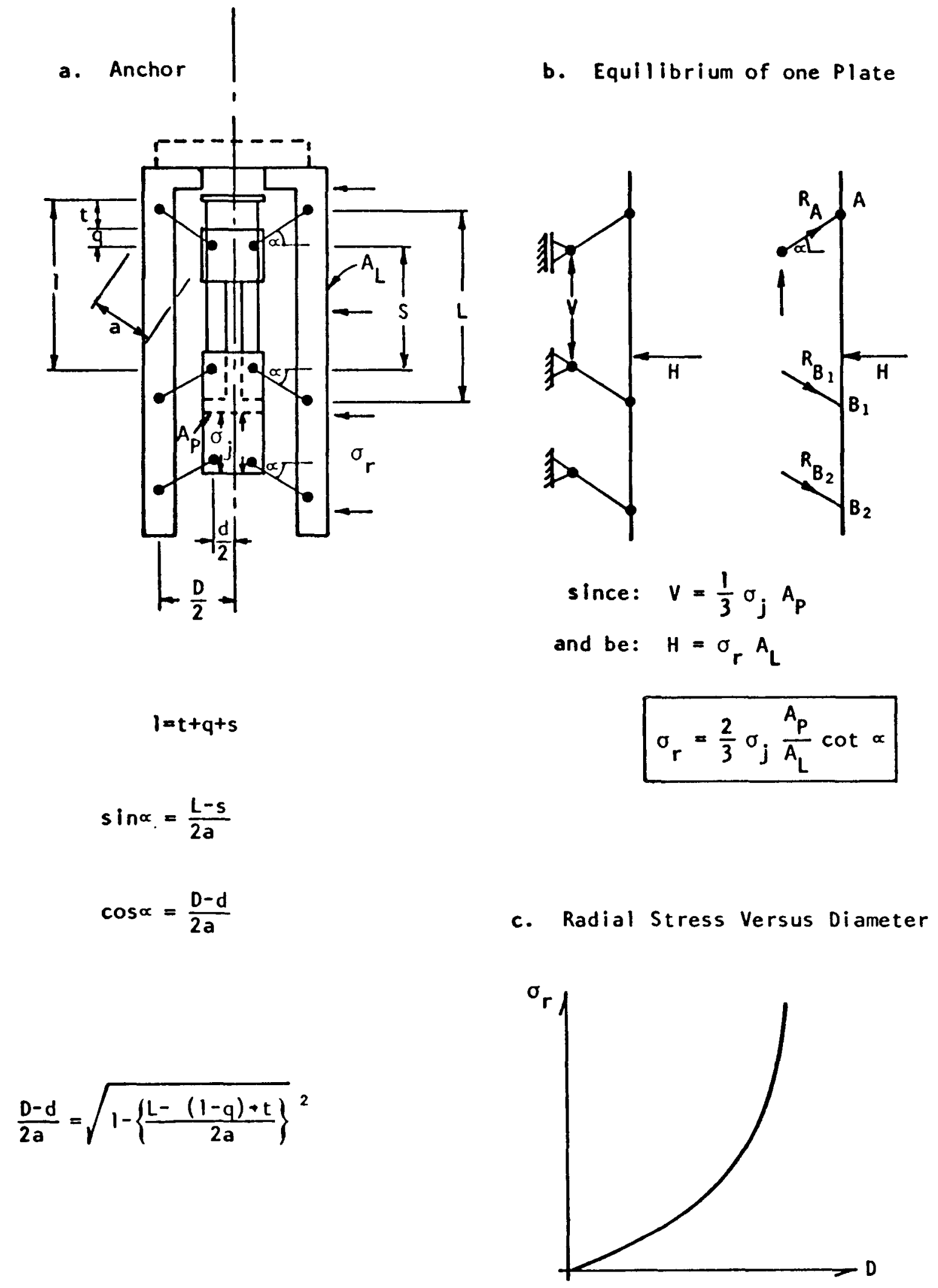

FIGURE 3-1. INSTALLATION OF THE ANCHOR. RELATIONSHIPS FOR MONITORING COUPLING 
where: $\quad \mathrm{D}=$ equivalent external diameter of the anchor

$t=$ complement of stroke displacement

$\mathrm{d}, \mathrm{a}, \mathrm{L}, \mathrm{l}, \mathrm{q}=$ constants of the anchor, as shown in Fig. 3-1

The radial stress applied by the anchor changes the state of stress of the soils in the vicinity of the anchor. Since the confining stress has an important influence on the modulus, (SW-AA, 1972) the numerical value of jacking stress is needed in interpreting the test data and accounting for this variation in stress conditions from the free field state of stress. The use for the radial stress is included in chapters 5 and 6 .

\subsection{ANALYSIS OF THE GENERATING STATION}

The generating station may be schematically represented as shown in Fig. 3-2. The purpose of this simplified representation is to describe the main elements of the dynamic system and to introduce the basic relationships of motion. The symbols in this figure are:

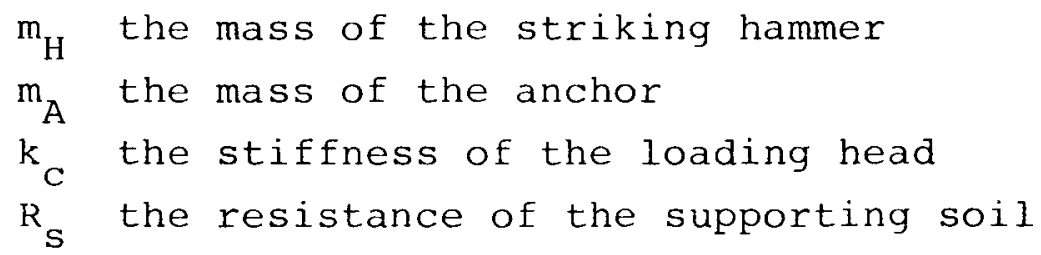

The impulse loading of the anchor, resulting from the impact applied by one blow of the striking hammer has an energy, $W$, given by:

$$
\mathrm{W}=\mathrm{m}_{\mathrm{H}} \mathrm{g} \mathrm{h}
$$

where: $h$ the height of free fall of the hammer

$g$ the acceleration of gravity 


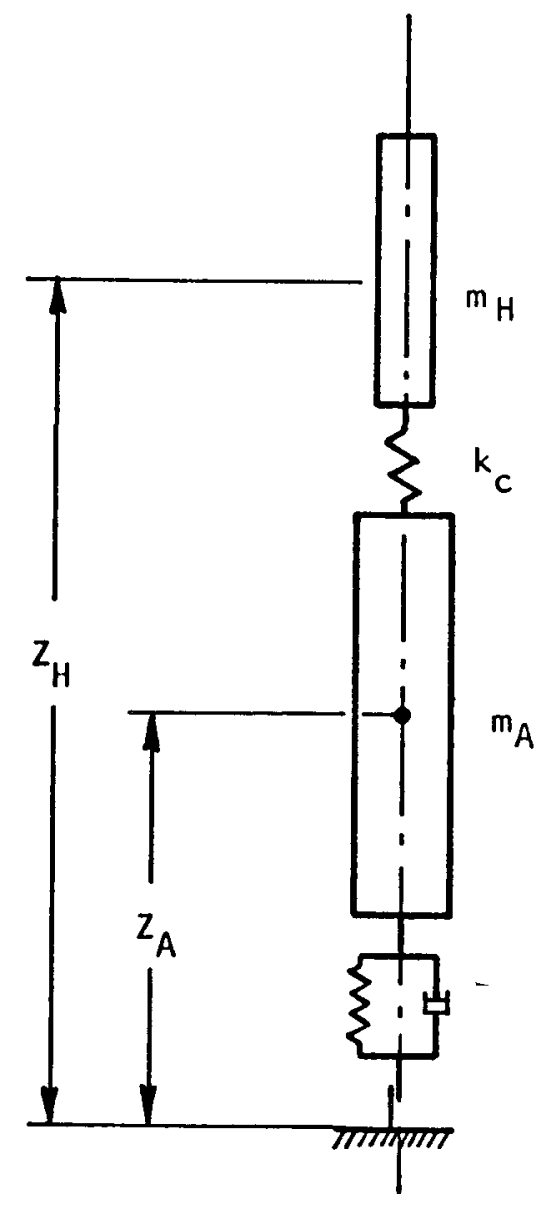

Free Body Equilibrium for Anchor:

$$
\begin{aligned}
& \underset{\text { Ampulse }}{\text { Applied }}\rfloor F(t)=k_{C}\left(Z_{H}-Z_{A}\right) \\
& \text { Inertia } m_{A} \ddot{z} \\
& \begin{array}{l|l}
\text { Soil } \\
\text { Resistance }
\end{array} \\
& \text { Equations of motion } \\
& \begin{array}{l}
m_{H} \ddot{Z}_{H}+k\left(Z_{H}-Z_{A}\right)=0 \\
m_{A} \ddot{Z}_{A}-k_{C}\left(Z_{H}-Z_{A}\right)+R_{S}=0
\end{array}
\end{aligned}
$$

FIGURE 3-2. SIMPLIFIED MODEL OF THE WIAVE GENERATING STATION 
The velocity of the hammer at the instant when it strikes the anchor assembly is:

$$
\mathrm{v}_{\mathrm{O}}=\sqrt{2 \mathrm{gh}}
$$

The applied force, as measured at the top of the anchor, is:

$$
F(t)=F_{1}[f(t)]=k_{C}\left(z_{H}-z_{A}\right)
$$

Where: $z_{H}$ and $z_{A}$ are the displacement time histories of the hammer and the anchor respectively, $F_{1}$ is the maximum applied force and $f(t)$ is a function which defines the shape of the load pulse.

The equations of motion of the simplified system, assuming 1) that the anchor and the hammer are rigid bodies, 2) that the loading head is a linear elastic element, and 3) that only vertical motions occur, are:

$$
\begin{aligned}
& m_{H} \ddot{z}_{H}+k_{C}\left(z_{H}-z_{A}\right)=0 \\
& m_{A} \ddot{z}_{A}+R_{S}(t)-k_{C}\left(z_{H}-z_{A}\right)=0
\end{aligned}
$$

In an actual test, the force $F(t)$ applied to the anchor is measured by means of a load cell attached to the striking plate. By substitution Equation 3.11 becomes:

$$
m_{A} \ddot{z}_{A}+R_{S}(t)-F(t)=0
$$

The resisting function, $\mathrm{R}_{\mathrm{S}}(t)$, represents the supporting force applied by the surrounding soil. Solution for this equation requires defining the resisting function in terms of the soil properties, the variables of motion, and the geometry of the 
problem. Although mechanical models exist for the behavior of the soil in terms of stiffness and viscous damping, it is not possible to determine the values of the corresponding constants which pertain to the boundary conditions of the problem (i.e., a short segment of a long cylindrical opening in a semi-infinite mass). The general problem is further complicated when considering the changed state of stress created in the surrounding soil by applying large radial stresses with the anchor. As discussed in tne previous section, the different degrees of coupling can also result in the possibility of reaching failure condition, slippage at the anchor soil interface or local shear failure from excessive jack pressures. All of these variables make it difficult to model "real" conditions at the generating station.

Because of the impossibility of finding a closed form solution to analyze the response of the anchor in the in situ test, finite element methods and special experimental tests have been employed for the development of the procedures for data interpretation. The results of this work are treated in detail in Chapters 4 and 5 .

\subsection{DISCUSSION OF WAVE PROPAGATION PHENOMENA}

Anchor motion, excited by a falling hammer (see Fig. 1-1), is transmitted as transient stress waves to the three-dimensional soil mass. These stress waves can exist as either P-waves or S-waves. P-waves (also termed primary or compressional waves) are characterized by particle motions along the direction in which the wave propagates, while S-waves (also termed secondary or shear waves) involve particle motions transverse to the direction of wave propagation. The anchor constitutes the origin of the disturbance during the in situ test; the direction of propagation for an element of soil, therefore, is approximately aligned with the line connecting the element with the anchor, provided the soil mass is locally homogeneous. Although the 
design of the anchor, shown schematically in Fig. 3-1, results in the generation of some horizontal motions in the soil, the predominant soil response is expected to be vertical. For soil elements located on the midplane of the anchor, therefore, for which the direction of propagation is horizontal and the predominant direction of particle motion is vertical, the s-wave component of the propagating stress wave should have a larger amplitude than the P-wave component, even though the P-waves may still be significant in the near-anchor region (Chapter 5). Furthermore, since S-waves and P-waves propagate at different characteristic wave velocities, the two components will separate at a distance from the anchor which depends on the two characteristic wave velocities and the duration and magnitude of the impulse load applied to the anchor.* Beyond this distance the $S$-wave component of the propagating stress wave is unaffected by the P-wave component.

The task of determining the strain-dependent in situ properties of the soil medium from a finite number of free-field particle velocity records requires that shear wave velocity and shear strain be determined from these records. Since the S-wave propagates at the shear wave velocity, a first approximation to the shear wave velocity of the soil medium can be obtained by observing the propagation of any identifiable feature of the $S$-wave. Due to dispersion of the $S$-wave as it propagates away from its nearly axisymmetric source, the various identifiable features do not all propagate at the same velocity as the wave itself. Furthermore, since the properties of the soil medium are strain-dependent, the shear wave velocity of an element of soil varies from instant to instant as the stress wave propagates through. For these reasons, the value of shear wave velocity obtained by observing a particular feature of the

For the special field tests described in chapter 5, the distance was about 2 feet. 
propagating $S$-wave is not necessarily related to the straindependent soil properties in a straightforward way. Therefore, special studies have been carried out, using finite element procedures (Chapter 4) and special field tests (Chapter 5) to investigate this problem.

Once the shear wave velocity has been determined, a first approximation of the peak shear strain occurring in a given element of soil can be obtained through the relation

$$
\gamma=\left|\frac{\dot{\mathrm{w}}}{\mathrm{V}_{\mathrm{S}}}\right|
$$

in which $\gamma$ is the magnitude of the peak shear strain, $\dot{w}$ is the peak particle velocity of the soil element, and $V_{S}$ is the shear wave velocity. Equation 3.13 was originally developed for the case of plane S-waves propagating in an infinite elastic medium. 'l'his relation can be shown to be valid for elements at large distances from the anchor for an in situ test. This can be done using a solution developed by C. C. Mow, 1965, to determine the free-field response of an infinite elastic medium to the arbitrary motion of a rigid spherical inclusion along a vertical axis. Equation 3.13 holds, in this case, for elements located along the midplane of the sphere at large distances from the sphere. At these large distances, the shape of the anchor (i.e., whether cylindrical or spherical) is not particularly important; furthermore, the shear strains in soil elements located at large distances from an actual anchor in an in situ test will be sufficiently small so that these particular elements can be considered to behave elastically. Therefore, the results from the above indicated closed form solution can be considered to be representative of the behavior of actual soil elements located at large radial distances from the anchor. For these particular elements, Equation 3.13 was shown to be applicable, as indicated above. 
The behavior of soil elements located in the immediate vicinity of the anchor is quite different from that represented by Equation 3.13. For such elements in an infinite elastic medium, the response may be more nearly represented by cylinarical waves; however in an in situ test of actual soil materials these waves are further complicated by the boundary effects at the anchor-soil interface (i.e., slippage, jacking pressures, the finite length of the anchor, etc.) and the inelastic behavior of the near-anchor soil elements. Such complex response characteristics cannot possibly be represented by closed form solution based on elementary wave propagation principles, as described above. However, these near-anchor response characteristics can be investigated by using nonlinear finite element techniques and by special field test procedures, as described in Chapters 4 and 5, respectively. 


\subsection{INTRODUCTION}

The in situ test to determine the shear properties of soils has undergone considerable development in previous efforts; however, various aspects of the data reduction procedures for these tests still require further evaluation. For example, current procedures for defining shear wave velocities between adjacent sensors have not yet been fully verified. In addition, current techniques for establishing shear strains from the measured soil motions are based on certain assumptions that require further assessment when applied to the complex response conditions that exist during the in situ test.

\subsubsection{Purpose of Calculations}

The purpose of the finite element calculations described in this chapter is to evaluate procedures for obtaining in situ soil properties from the test data. This is carried out by treating each nodal point in the finite element grid as a sensor, and the velocity histories obtained from the calculations as field measurements. Data processing procedures used to estimate strain-dependent shear moduli from actual field data are applied to these computed velocity histories. The shear moduli estimates obtained using these procedures are then compared to the shear moduli actually used as input to the finite element model.

The use of finite element calculations in this manner offers several important advantages when assessing data processing procedures. First of all, the soil properties incorporated as input data for the finite element model provide a basis for evaluation of the results of the various data processing methods. 
In addition, the closely spaced nodal points that comprise the finite element grid represent a much finer network of soil response "sensors" than is ordinarily available under field production test conditions. Finally, these nodal points monitor the soil response not only in the vicinity of the middepth of the anchor, as per the actual field tests, but throughout a two-dimensional array of locations encompassing the entire nearanchor region of soil.

While the above advantages show the finite element approach to be a potentially powerful means of assessing the data processing procedures, there are also certain limitations that must be considered. These are associated with the fact that it is impractical to incorporate every detail of an actual in situ test into the finite element model. For example, the details of the anchor, including the nonaxisymmetric bearing of the individual plates against the sides of the borehole, have been omitted from the finite element calculations discussed herein. In addition, the effects of the jacking pressure and possible slippage of the anchor along its interface with the soil have not been considered. Such limitations in the finite element model do not represent deficiencies in the method itself; rather they have been introduced to keep computational costs within reason. It is anticipated that the inaccuracies arising due to these limitations are small and confined to the immediate vicinity of the anchor. Furthermore, slight differences between calculation and experiment are not severely detrimental to the goal of evaluating data processing techniques. The evaluation procedure is entirely self-contained within the finite element calculation, and is independent of experimental results. It is for the purpose of extrapolating conclusions from analytical checkout into actual field practice that good correlation. between calculation and experiment is desirable. 
Once dynamic soil responses are measured during the in situ tests, data processing techniques must be used to convert these response measurements to corresponding dynamic soil properties in shear. The principal objective, that of determining a relationship between shear modulus and level of shear strain in the in situ medium, involves two phases of data interpretation. First, the shear wave velocity, which determines the shear modulus, must be obtained; and then the strain level corresponding to that value of shear wave velocity must be determined. Initially, it is best to consider these phases separately; however, before the final recommendations can be formulated, the various methods for estimating shear moduli and strains, by themselves, must be tested in combinations suitable for the construction of modulus/strain relationships. It is on the basis of how well these modulus/strain relationships approximate the known relationships that the recommendations will be made. Two calculations have been included in the present study: one in which the soil has been modeled as a linear elastic medium, and one in which the soil has been described as a nonlinear, hysteretic material.

The elastic finite element calculation is of great value in assessing each phase of data reduction separately. The shear wave velocity is constant in this case, thus simplifying the evaluation of those data reduction techniques intended to determine shear wave velocity. Furthermore, since the method of estimating shear strain depends on the shear wave velocity, an a priori knowledge of shear wave velocity uncouples the strain calculation from the shear-wave-velocity phase of the data reduction. Any problems encountered in estimating shear strain, therefore, can be traced to the shear-strain phase of the data reduction. 
The nonlinear finite-element calculation allows the various data processing methods to be applied under conditions more nearly simulating the actual conditions of an in situ test. In fact, the nonlinear soil properties, hammer weight, Belleville spring constant, and anchor weight used in the nonlinear calculation correspond as nearly as possible to those parameters from the experimental tests in chapter 5. Therefore the nonlinear finite element results can be compared directly to the special test results (Section 4.3.1) and can serve to provide further insight into results from that test program. In addition, the nonlinear finite element calculation is of value in evaluating both phases of data reduction acting together. In the elastic case, the shear wave velocity is independent of strain level, making it meaningless to attempt to construct a modulus/strain relationship. In the nonlinear case, however, a nontrivial modulus/strain relationship does exist and, more importantly, is known from the input data to the calculation. Because of this, combinations of data reauction techniques intended to determine the modulus/strain relationship can be verified against the input data.

\subsection{FINITE ELEMENT MODEL}

I'he calculations described in this chapter were carried out using FEDIA, a dynamic, two-dimensional, continuum finite element computer code developed at AA. The material properties that can be accommodated in FEDIA range from linear elastic to highly complex nonlinear properties representative of soils or reinforced concrete and based on variable bulk and/or shear moduli and plastic yield functions. The boundary conditions available include time-varying pressures, time-varying velocities, fixed or free conditions along either global axis, and energy absorbing boundaries. The memory usage is dynamically allocated, and when necessary, data is stored on peripheral units so that larger problems may be solved than would otherwise be possible. 


\subsubsection{Finite Element Grid}

The finite element grid used in both the elastic and the nonlinear calculations is shown in Fig. 4-1. The grid is axisymmetric and contains a total of 954 elements and 1020 nodal points, with the greatest mesh refinement in the vicinity of the anchor. The upper boundary of the grid is modeled as a free surface, 20 feet above the center of the anchor. To simulate a soil medium of infinite extent, the bottom and right boundaries were assigned energy-absorbing properties, thereby minimizing any signals reflecting back into the interior of the grid from these boundary locations. The left boundary of the grid, which corresponds to the borehole, was subjected to stressfree boundary conditions along that portion of the hole wall not in contact with the anchor.

It can be seen from Fig. 4-1 that the finite element grid employs an especially fine array of elements and nodal points in the vicinity of the anchor, which is the region of primary interest in both of these calculations. The nodal-point spacing is as small as 2 inches at the anchor/soil interface, and gradually increases with increasing distance from the anchor to a maximum of 24 inches at the right boundary of the grid. However, the spacing never exceeds 10 inches within 10 feet of the anchor/soil interface.

The ability of the grid to accurately transmit shear waves depends on the nodal-point spacing and the shear wave velocity of the soil medium. In the elastic calculation, in which the shear wave velocity is $1000 \mathrm{fps}$, the fineness of the grid allows the undistorted transmission of shear waves with 


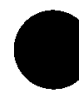


a frequency content of up to $500 \mathrm{~Hz}$ out to a distance of 5 feet from the anchor. Furthermore, in the near-anchor region, shear waves with frequency components of up to $1500 \mathrm{~Hz}$ can be propagated without distortion. In the nonlinear calculation, in which the shear wave velocity varies but is typically between 400 and $500 \mathrm{fps}$, shear waves having frequencies of up to $200 \mathrm{~Hz}$ can be accurately transmitted to a distance of 5 feet from the anchor and signals of up to $600 \mathrm{~Hz}$ are permissible near the anchor. Typical free-field velocity histories for a soil medium in which the material properties are virtually the same as those of the nonlinear finite element calculation are available from the special tests. The upper bounds of the frequencies contained in these velocity histories are about $300 \mathrm{~Hz}$ near the anchor and about $200 \mathrm{~Hz}$ at about 5 feet from the anchor. These values are well within the transmission capabilities of the finite element grid.

\subsubsection{Application of Dynamic Loading}

In the in situ test, loads are applied by means of a freely falling hammer, which strikes Belleville springs attached to the top of a relatively rigid anchor. The hammer, Belleville springs, anchor, and soil then form a coupled dynamic system through which loads are applied to the soil along the vertical anchor/soil interface. As shown in Fig. 4-2, this dynamic system is incorporated as completely as possible into the finite

It is assumed that good resolution of a frequency component of a shear wave is assured if the ratio of wave length, $L$, to mesh size equals or exceeds 4 . The highest frequency component compatible with a given nodal-point spacing, $a$, can then be computed from the formula

$$
f=\frac{V_{s}}{L}=\frac{V_{s}}{4 d}
$$

where $V_{S}$ is the shear wave velocity of the medium, and $f$ is the maximum frequency in $\mathrm{Hz}$. 


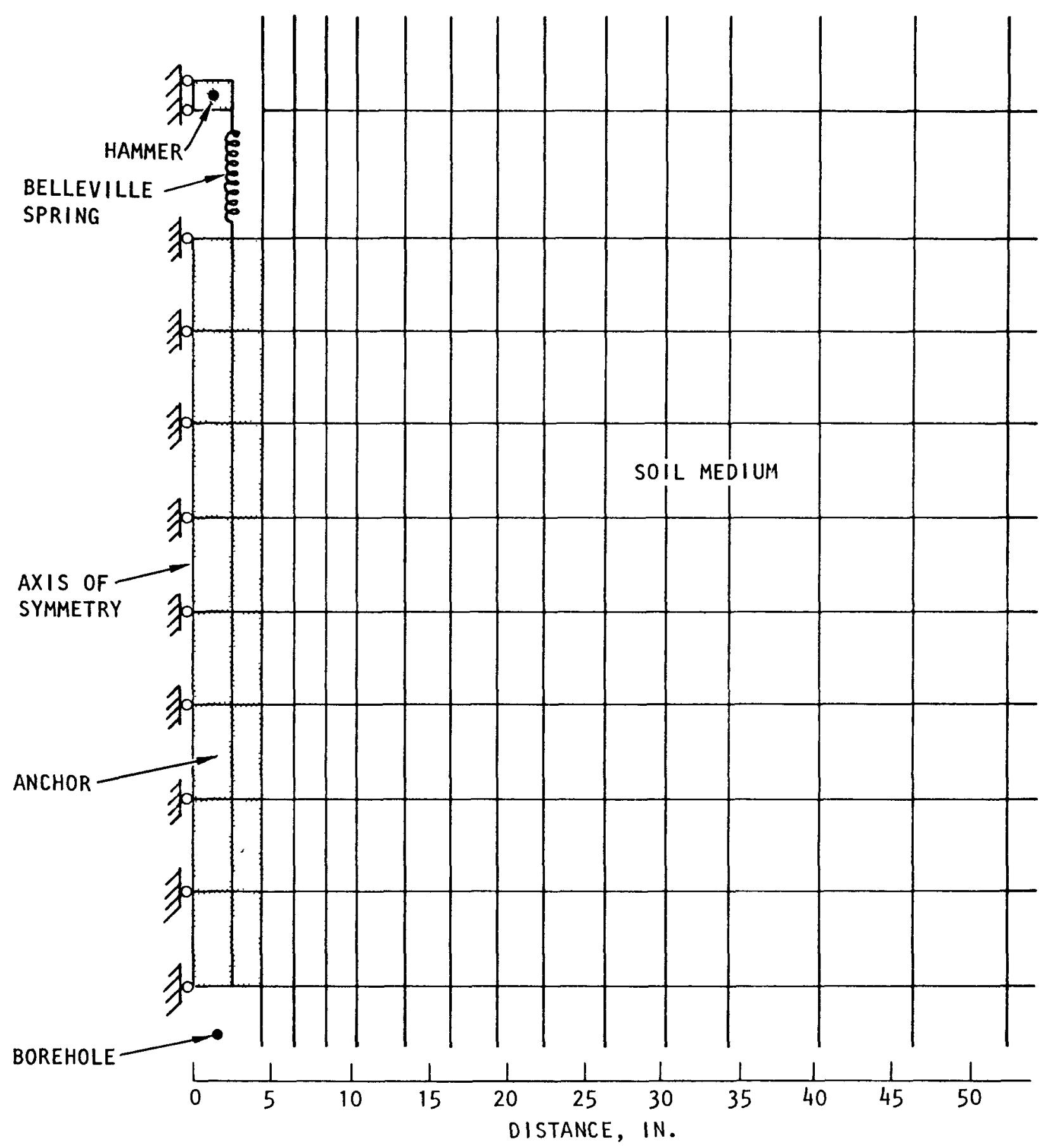

FIGURE 4-2. ENLARGED VIEW OF THE NEAR-ANCHOR REGION OF GRID 
element model. The Belleville springs are represented as a one-dimensional massless element with a finite compression stiffness of $322 \mathrm{kip} / \mathrm{in}$. This element has no tension stiffness in order to allow for possible uplift of the hammer. The top of the spring element of Fig. 4-2 is connected to a single element which simulates the dropped hammer. The weight of this element was taken to be 150 lb for the elastic calculation, and $58 \mathrm{lb}$ for the nonlinear calculation. The anchor is modeled using axisymmetric elements that are very stiff when compared to the soil. The total weight of these elements corresponds to the actual weight of the anchor (195 lb). The anchor elements are considered to be fully bonded to the adjacent soil elements.

Time-zero of each calculation is taken to be the time at which the freely falling hammer makes initial contact with the Belleville spring. The drop height of the hammer is entered into the calculation in the form of an initial velocity corresponding to the velocity of the hammer immediately prior to initial contact with the Belleville spring when released from the specified drop height. The initial conditions are as follows :

$$
\begin{aligned}
& x(0)=0 \\
& \dot{x}(0)=\sqrt{2 g h}
\end{aligned}
$$

where: $\quad x(0), \dot{x}(0)=$ initial displacement and velocity of the hammer

$$
\begin{aligned}
g= & \text { acceleration due to gravity }= \\
& 32.2 \mathrm{ft} / \mathrm{sec}^{2} \\
\mathrm{~h}= & \text { height above Belleville spring from } \\
& \text { which hammer is dropped }
\end{aligned}
$$

In the elastic case the drop height was 2 feet, resulting in an initial velocity of $11.35 \mathrm{fps}$. In the nonlinear case the 
drop height was 6 inches, corresponding to an initial velocity of 5.67 fps.

\subsubsection{Representation of Soil Materials}

The properties of the soil in the elastic finite element calculation are as follows: the shear wave velocity is 1000 fps, Poisson's ratio is 0.3 , and the unit weight is 100 pcf. The material model used in the nonlinear finite element calculation was designed to fit cyclic triaxial test data obtained from the sand used in the special pit tests. The material model used is of the variable-modulus type in which the instantaneous tangent shear and bulk moduli are computed, for each integration step of the calculation, as a function of the current state of stress and the stress history of each element of the grid. Hysteresis is incorporated into the shear modulus by distinguishing, on the basis of stress history, between the conditions of virgin loading and of unloading/reloading. Virgin loading exists if the current principal component of shear stress exceeds all previous values. Otherwise the element is undergoing unloading/reloading. A thorough description of the soil model, along with numerical values of the parameters used and curves comparing the model with the cyclic triaxial data, is contained in Appendix A.

\subsubsection{Integration Time step}

The integration time-step schedule for the elastic calculation is $0.05 \mathrm{msec}$ for the first $7 \mathrm{msec}, 0.1 \mathrm{msec}$ for the next $5 \mathrm{msec}$, and $0.2 \mathrm{msec}$ thereafter, resulting in a total record length of $20 \mathrm{msec}$. This time increment schedule is much finer than that required merely to satisfy the dynamic stability criteria of the finite element method. The fineness of the time increment arises from the necessity of knowing the time of arrival, at a particular location, of various key features of 
the response signature, such as the first peak of the particle velocity. In computing the travel times of these key features across distances as small as 2 inches, for which the transit time of a pulse propagating at $1000 \mathrm{fps}$ is $0.167 \mathrm{msec}$, it is necessary to know the arrival times of these features to within a margin of error which is small compared to this transit time. While the time increment of $0.05 \mathrm{msec}$ may not be small enough to allow satisfactory resolution over a 2 inch interval, interpolation techniques can be applied to the data, if necessary, to improve the resolution.

A similar line of reasoning was used to select a timeincrement schedule for the nonlinear calculation. Although the shear wave velocity in this case is not constant, its average value is about half as large as the shear wave velocity of the elastic calculation. Therefore transit times between adjacent nodal points are approximately twice as long in the nonlinear calculation as in the elastic case. For this reason the nonlinear calculation consisted of time increments twice the size of those of the elastic calculation. The schedule is as follows: 0.1 msec for the first $14 \mathrm{msec}, 0.2 \mathrm{msec}$ for the next $10 \mathrm{msec}$, and $0.4 \mathrm{msec}$ thereafter, resulting in a total time span of $40 \mathrm{msec}$.

\subsection{RESULTS OF ANALYSIS}

Results of the dynamic analyses are obtained in the form of motion time histories for each nodal point in the grid, and stress and strain time histories for each element. As noted in section 4.1.1, these results are used to assess data processing techniques currently used in the in situ soil test program. In addition, the results are used to study the characteristics of the waveforms generated in the soil during the in situ tests. These assessments and studies are summarized in the remainder of this section. 
It was pointed out in Section 4.1.1 that the finite element calculations can be used to evaluate the data processing procedures without comparing the calculations with experimental results. In fact the elastic calculation is used exactly this way, in that there has been no experiment conducted in an elastic medium. However, since the nonlinear finite element calculation more nearly simulates the special test conditions, its comparison with experimental results is possible. The application of the results of the evaluations of data processing procedures to the processing of actual field data would be greatly facilitated if good correlation between calculation and experiment were to be obtained. For the sake of credibility, therefore, it seems appropriate that the discussion of the analytical results should begin with a comparison of the nonlinear finite element results with the results from the special experimental pit tests. The sensors from the special pit tests used in this comparison are shown in Fig. 4-3.

The velocity histories recorded from the special pit tests were obtained using velocity transducers having the frequencyresponse spectrum of Fig. 4-4. The experimental displacement histories were obtained by integration of the velocity histories. In order to provide a consistent comparison between calculation and experiment, it is necessary to adjust the calculated freefield response in a manner that simulates the effect of the transducers. This is accomplished by operating on the calculated velocities with a transfer function having the frequency response of Fig. 4-4. Adjusted displacements are then obtained from the adjusted velocities by integration. Both the original calculated response and the adjusted response are shown in the comparison plots which follow. 


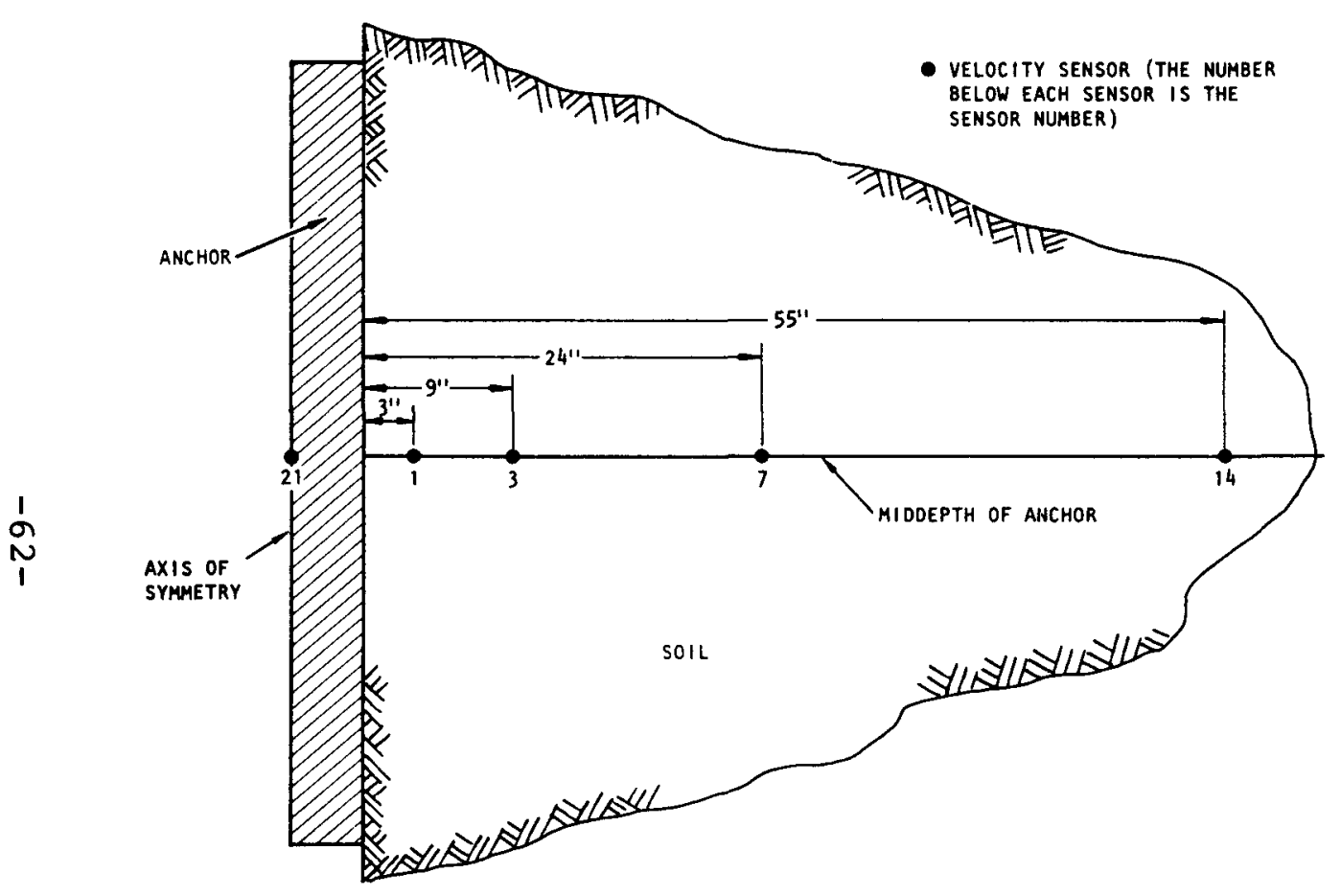

FIGURE 4-3. NOMINAL LOCATIONS OF SPECIAL TEST VELOCITY SENSORS USED IN COMPARISON WITH CALCULATED RESULTS

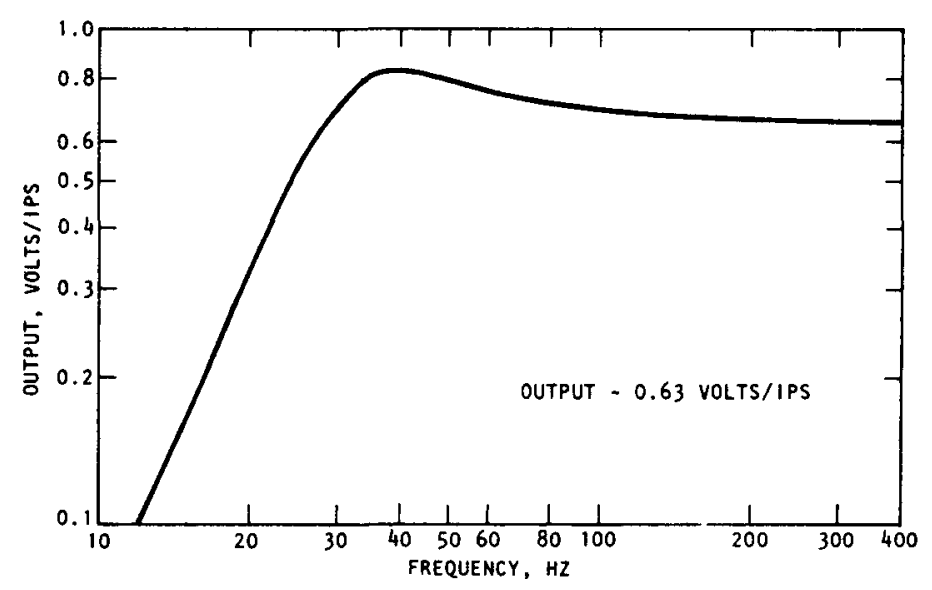

FIGURE 4-4. FREQUENCY-DEPENDENT OUTPUT FROM GEOPHONE -- SEW/AA FY 75 FIELD TESTS 
Figs. 4-5 to 4-9 show comparisons between the experimental and the calculated response during an in situ test. Since there is no way to correlate the starting time of the experimental time histories with the starting time of the calculated time histories, arbitrary time shifts have been employed. For any given nodal point, the velocity and displacement histories have been shifted by equal amounts. The experimental results, plotted as solid lines, correspond to mean response curves taken over several repetitions of the test. The calculated results shown are from nodal points located the same distances from the anchor as the sensors to which they are being compared. In cases where no nodal point occurs at the approximate location of a particular sensor, responses of the two nearest neighboring nodal points on either side of the actual sensor location are snown.

Comparisons of the unadjusted calculated responses with the experimental responses indicate an excellent agreement among the velocities. The computed peaks tend to be slightly low at the anchor and slightly high at large distances from the anchor, suggesting that the calculation may have slightly underestimated the dissipative properties of the soil. But the general form of the calculated velocity response appears to be accurate throighout the entire time span of the experiment. The displacement histories show less agreement. A noticeable difference between calculation and experiment involves the occurrence of permanent displacements in the near-anchor region of the finite element results. There is no evidence of permanent displacements in the experimental data.

Reasons for this difference between the computed and measured displacement histories can be attributed, at least in part, to the frequency characteristics of the sensor. As noted in Fig. 4-4, the sensor has extremely low output at low frequencies. 'This suggests that any permanent displacements, which essentially 


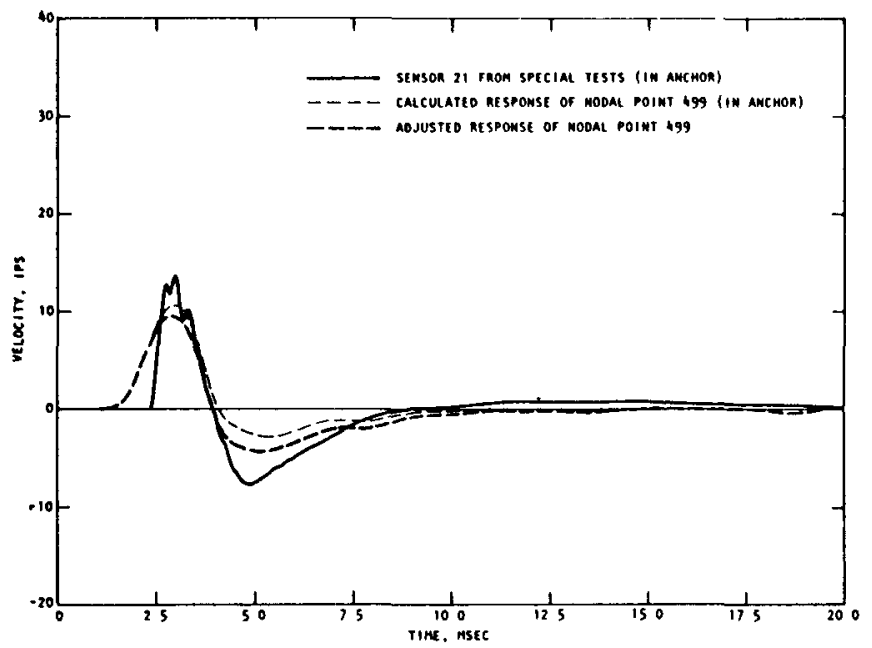

(a) Vertical velocity (positive downward)

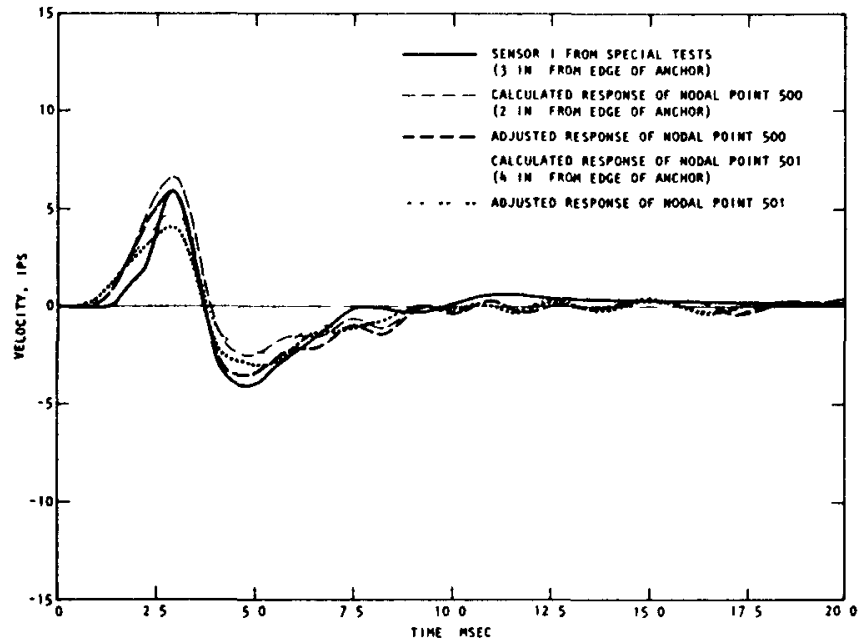

(a) Vertical velocity (positive downward)

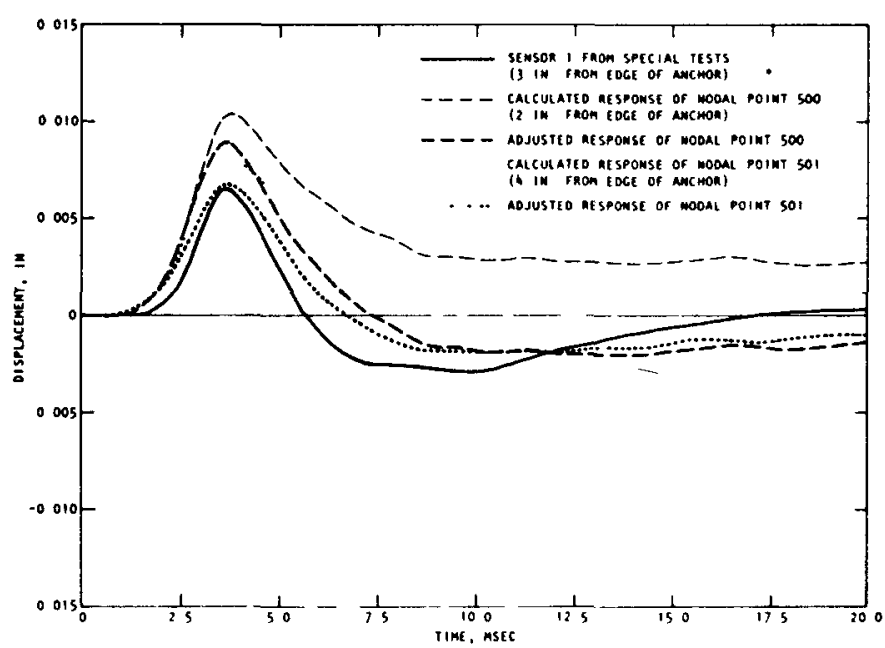

(b) Vertical displacement (positive downward)

FIGURE 4-6. COMPARISON BETWEEN EXPERIMENT AND
CALCULATION OF RESPONSE $3 \mathrm{IN}$. FROM EDGE OF ANCHOR

FIGURE 4-5. COMPARISON BETWEEN EXPERIMENT AND
CALCULATION OF ANCHOR RESPONSE

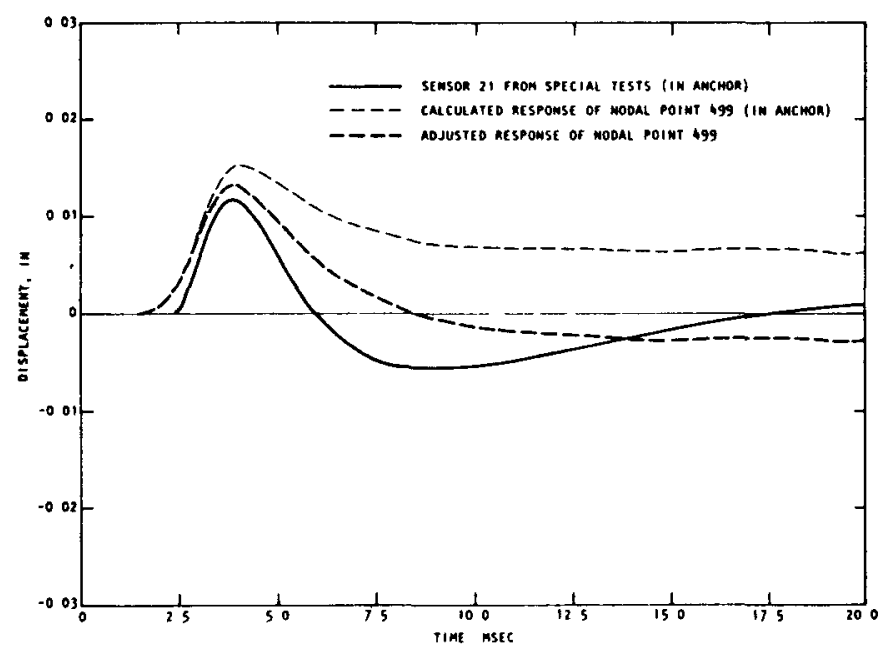

(b) Vertical displacement (positive downward)

\section{FIGURE 4-5. COMPARISON BETWEEN EXPERIMENT AND
CALCULATION OF ANCHOR RESPONSE}




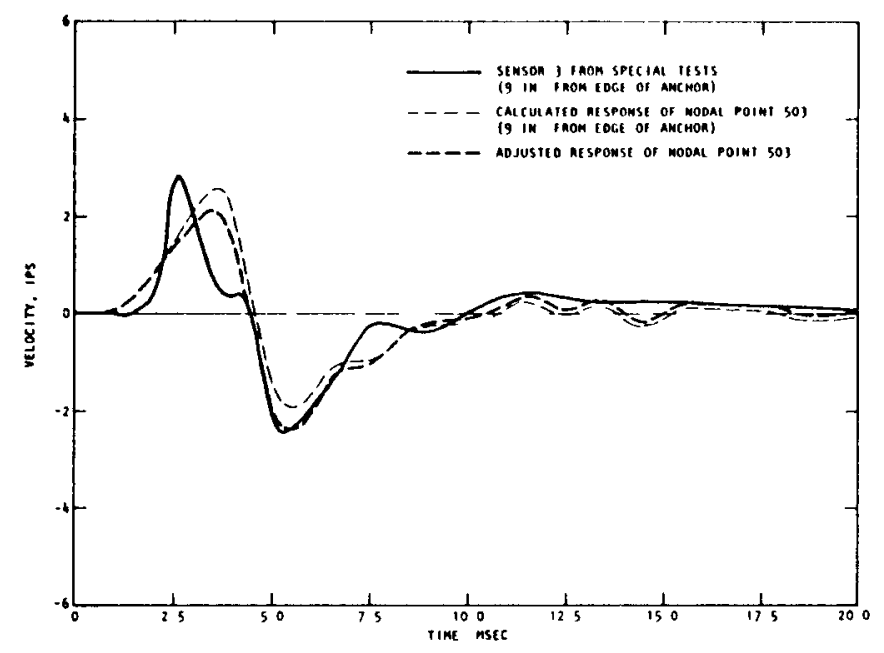

(a) Vertical velocity (positive downward)

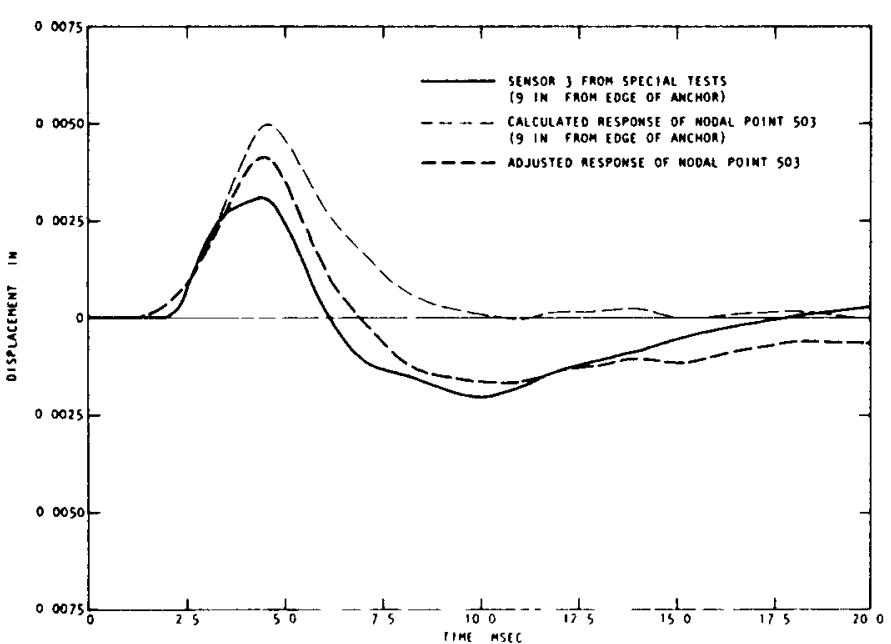

(b) Vertical displacement (positive downward)

FIGURE 4-7. COMPARISON BETWEEN EXPERIMENT AND CALCULATION OF RESPONSE $9 \mathrm{IN}$. FROM EDGE OF ANCHOR

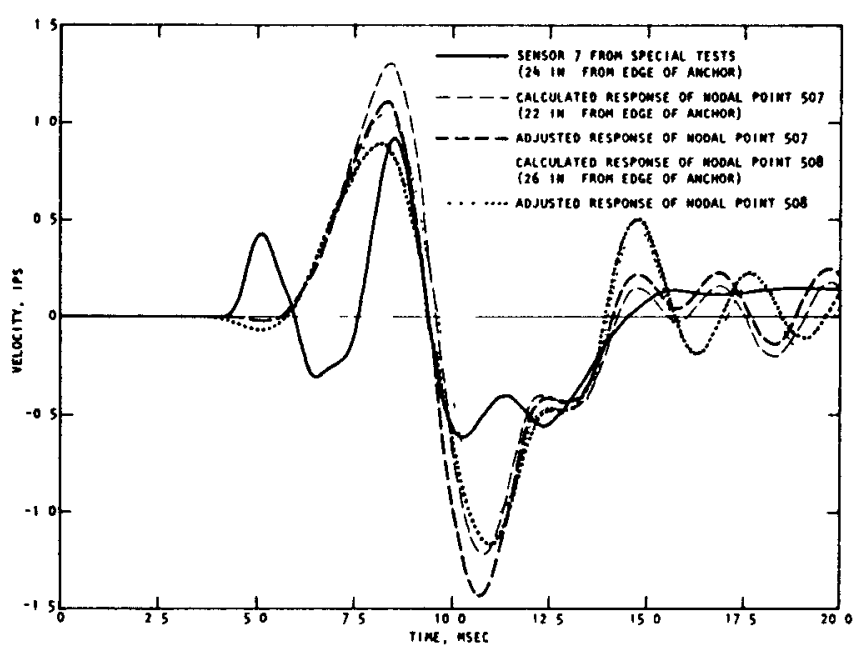

(a) Vertical velocity (positive downward)

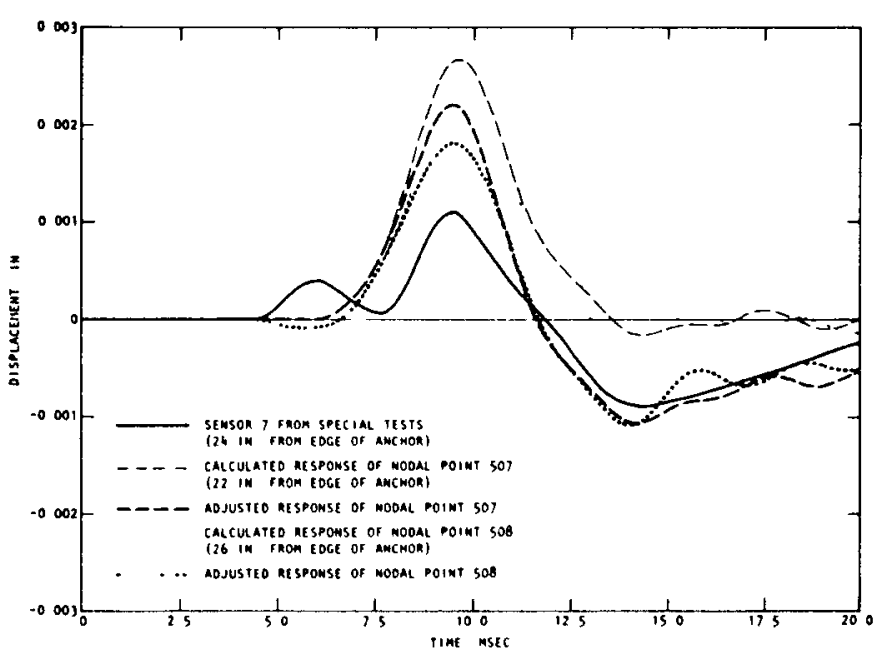

(b) Vertical displacement (positive downward)

FIGURE 4-8. COMPARISON BETWEEN EXPERIMENT AND CALCULATION OF RESPONSE 24 IN. FROM EDGE OF ANCHOR 


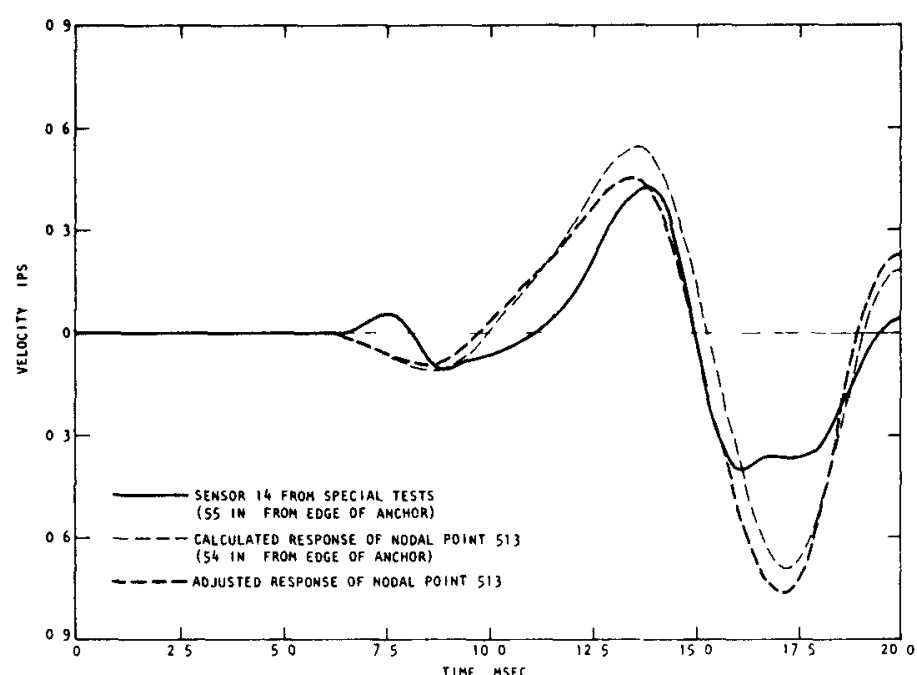

(a) Vertical velocity (positive downward)

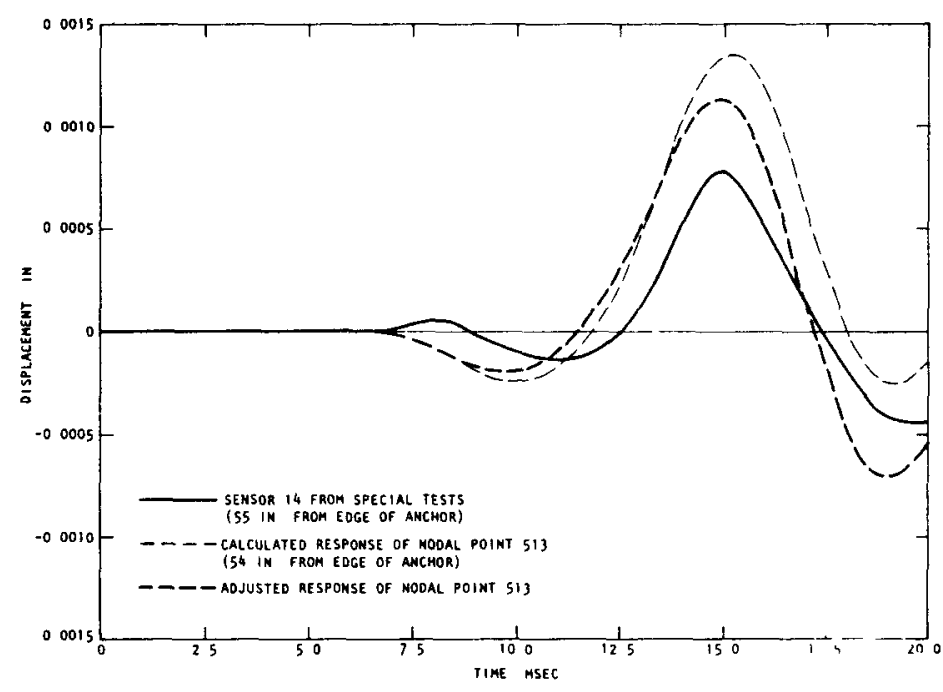

(b) Vertical displacement (positive downward)

FIGURE 4-9. COMPARISON BETWEEN EXPERIMENT AND CALCULATION OF RESPONSE 55 IN. FROM EDGE OF ANCHOR 
correspond to a zero-frequency response, are filtered out by the sensor. Therefore, it is most consistent to compare the experimental response histories with the computed response histories that have been adjusted to account for the presence of the sensor. These comparisons, as shown in Figs. 4-5 to 4-9, indicate a significantly improved correlation between the computed and measured displacement histories. In fact, it can be concluded that the adjusted finite element results provide a generally excellent comparison with the experimental results over the entire time frame of the computations. This high level of correlation justifies the application of the conclusions of this analytical study to actual field data.

Note that the adjusted aisplacements of the finite element calculation show no indication of permanent displacements, even though the unadjusted displacement histories show them to be present near the anchor. This shows that these velocity transulucers though all matched to respond the same, may not accurately measure permanent displacements, as explained above. This observation together with the observation that the adjusted finite element response has been quite successful at representing the transducer output, indicates that permanent displacements probably occur in the near-anchor region of actual in situ tests.

4.3.2 General Nature of Waves Generated During In Situ Soil Tests

The nature of the wave propagating away from the anchor is of great importance to the success of the in sicu soil test program. For example, if the shear wave velocity is to be successfully determined, it must be shown that the wave, or some identifiable portion of it, is a shear wave. Furthermore, the formulae used in determining the shear strain require the stress field to be nearly uniform in the vertical direction. since the finite element results contain a vast quantity of 
information pertaining to motions, stresses, and strains in the near-anchor region, systematic examination of these results can provide valuable insight as to the nature of the propagating wave.

Fig. 4-10 shows contour plots of the vertical component of particle velocity in the vicinity of the anchor at several instants of time. The contour plots show only the portion of the near-anchor region which lies above the middepth of the anchor. The velocity and stress fields shown in these figures are nearly symmetric with respect to the middepth. Essentially no information has been omitted, therefore, by showing only half of the near-anchor region. By comparing the position of the crest of the velocity wave of Fig. 4-10 with the position of the crest of the shear stress wave of Fig. 4-11, it can be seen that the two travel together. This indicates that the aisturbance identified in the response of the velocity transducers of an in situ soil test is especially rich in shear and its propagation is governed by the shear properties of the soil medium. Furthermore, both Figs. 4-10 and 4-1l show that the variation of velocity and stress in the vertical direction is negligible compared with the variation in the radial direction. This observation validates the assumption that only radial gradients of vertical displacenent need be considered in estimating shear strain.

Fig. 4-12 shows the shear stress vs. shear strain paths of several elements along the middepth of the anchor for the nonlinear finite element calculation. These elements are located at distances of 1 inch, 10.5 inches, 24 inches, and 57 inches from the edge of the anchor. The values of the secant shear modulus, $G$, and the damping ratio, $\lambda$, are shown for each element. It can be seen from this figure that both the modulus and the damping are significantly different for elements near the anchor, where strains are large, than for elements far from 


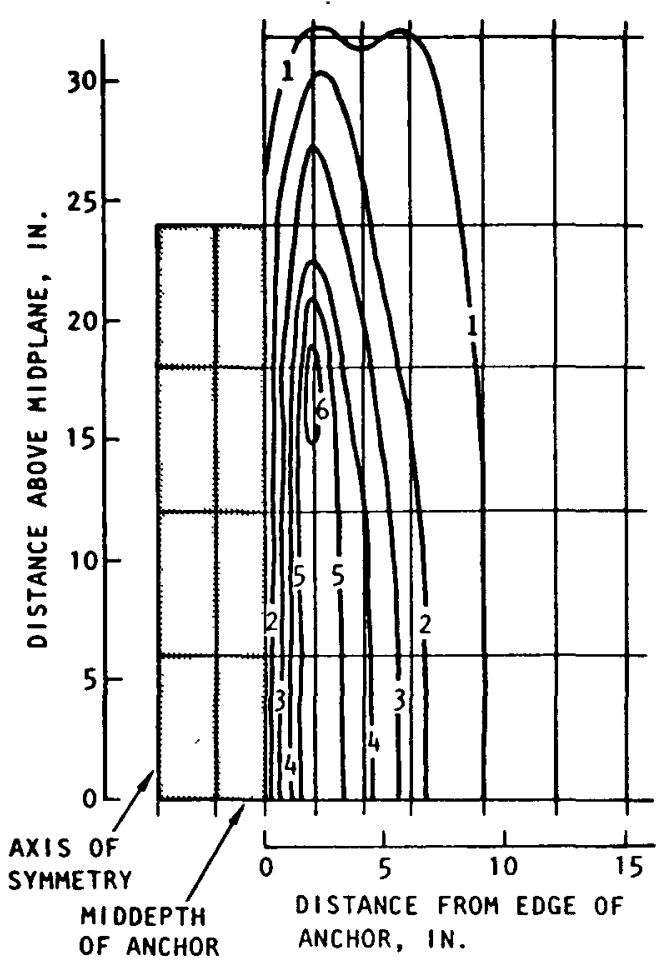

(a) $2.5 \mathrm{msec}$

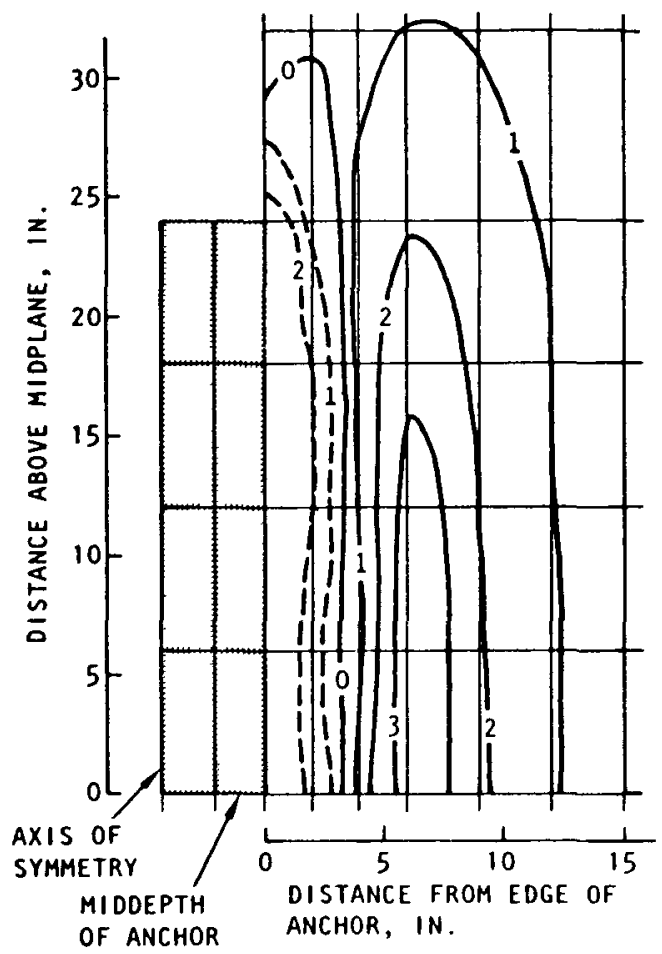

(c) $3.5 \mathrm{msec}$

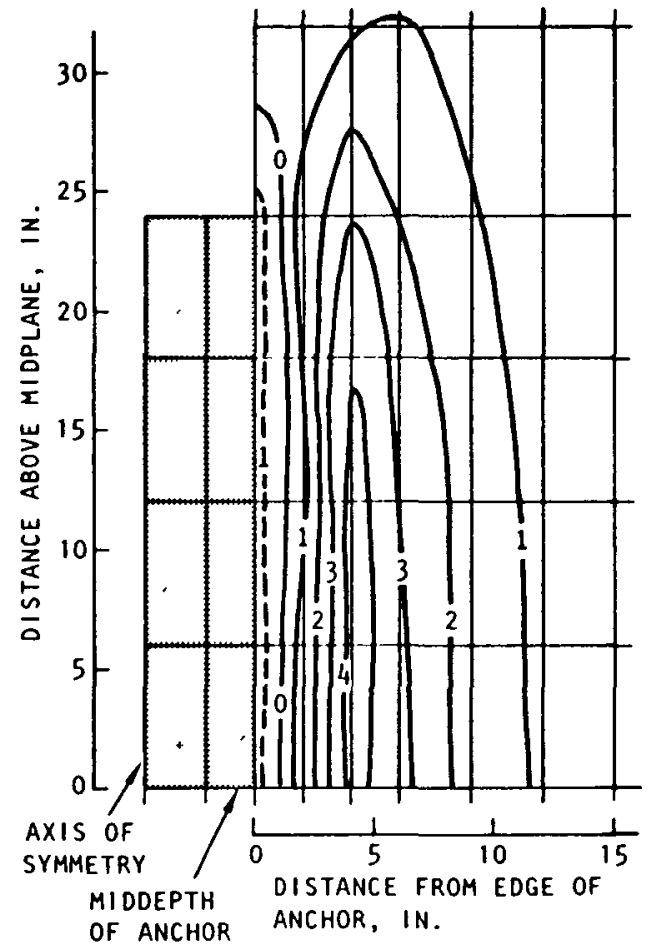

(b) $3.0 \mathrm{msec}$

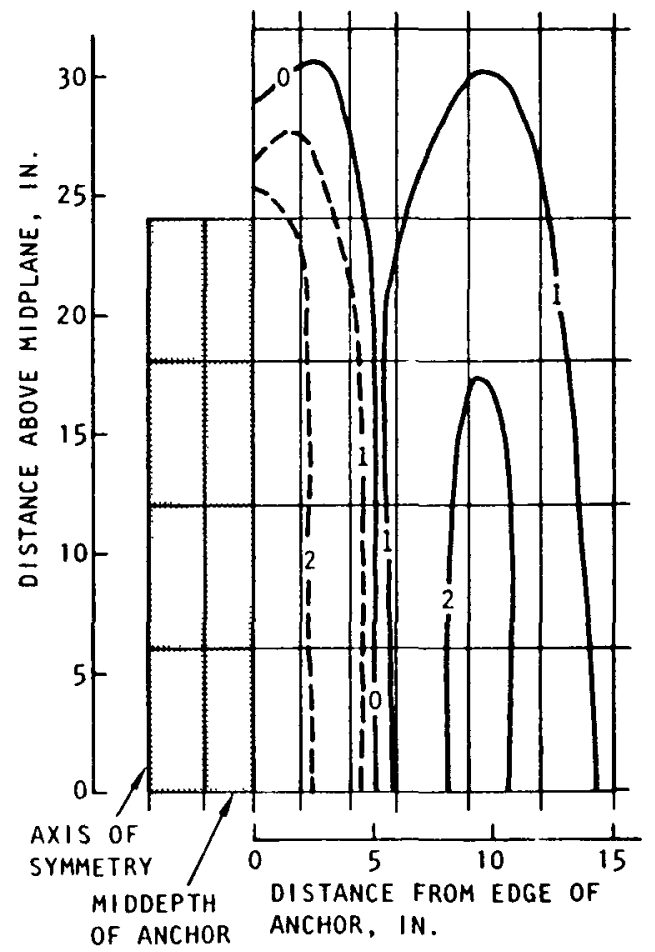

(d) $4.0 \mathrm{msec}$ 


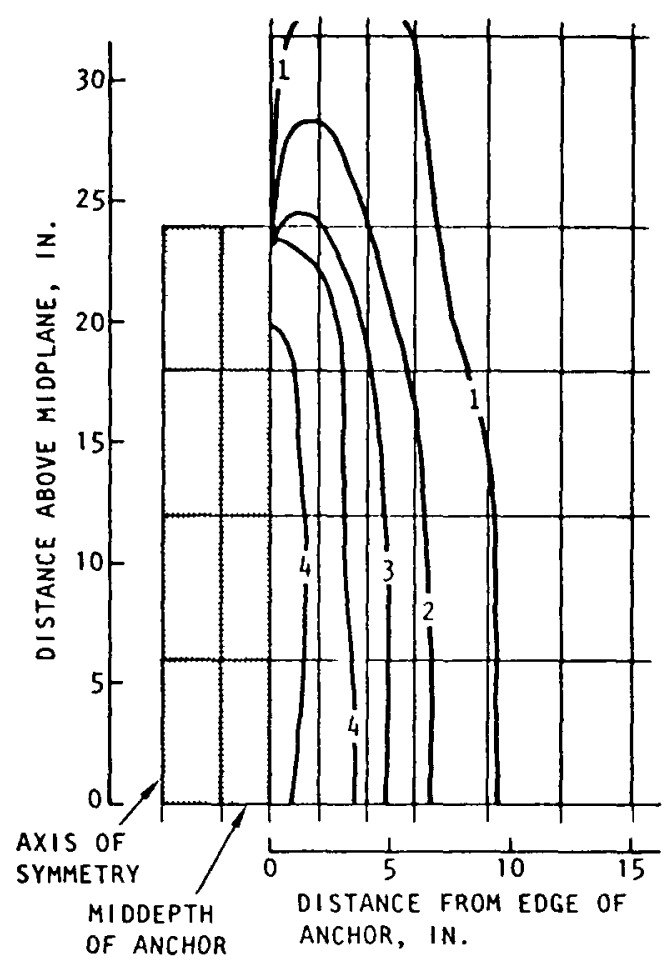

(a) $2.5 \mathrm{msec}$

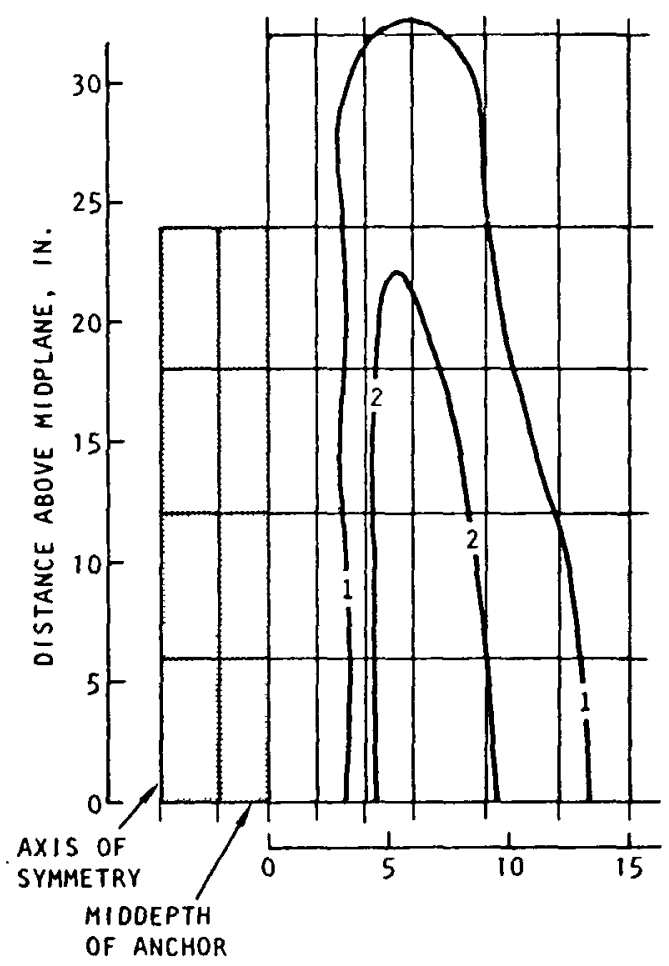

(c) $3.5 \mathrm{msec}$

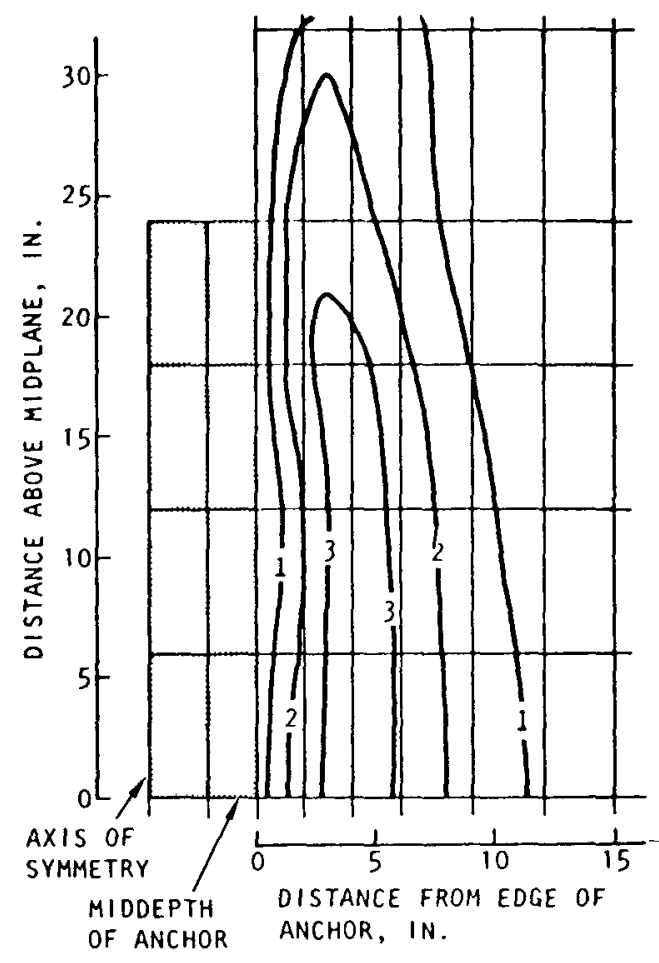

(b) $3.0 \mathrm{msec}$

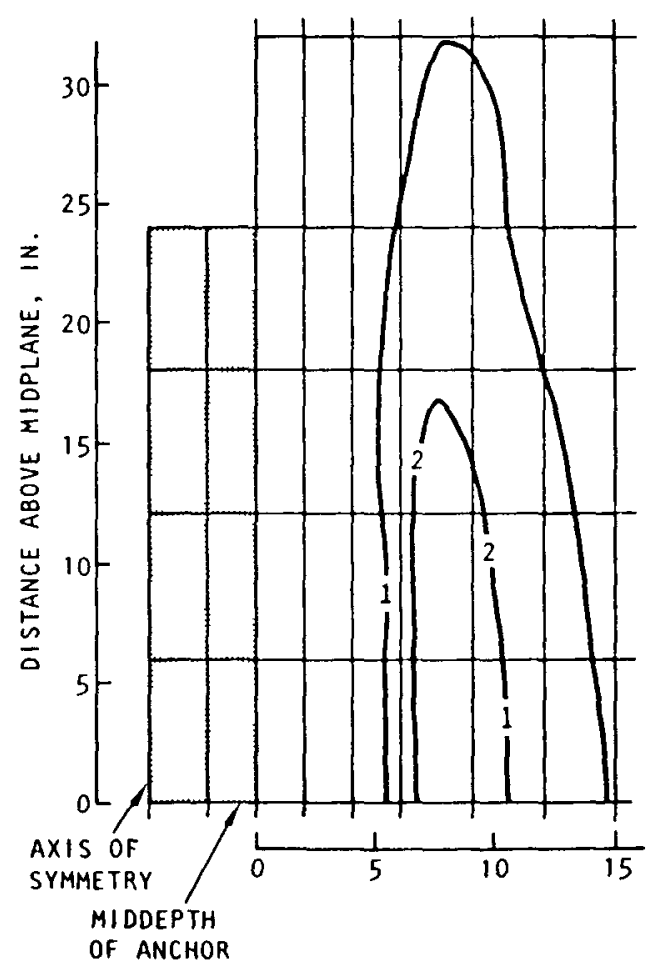

(d) $4.0 \mathrm{msec}$

FIGURE 4-11. CONTOUR PLOTS OF SHEAR STRESS (PSI) IN THE NEAR-ANCHOR REGION 


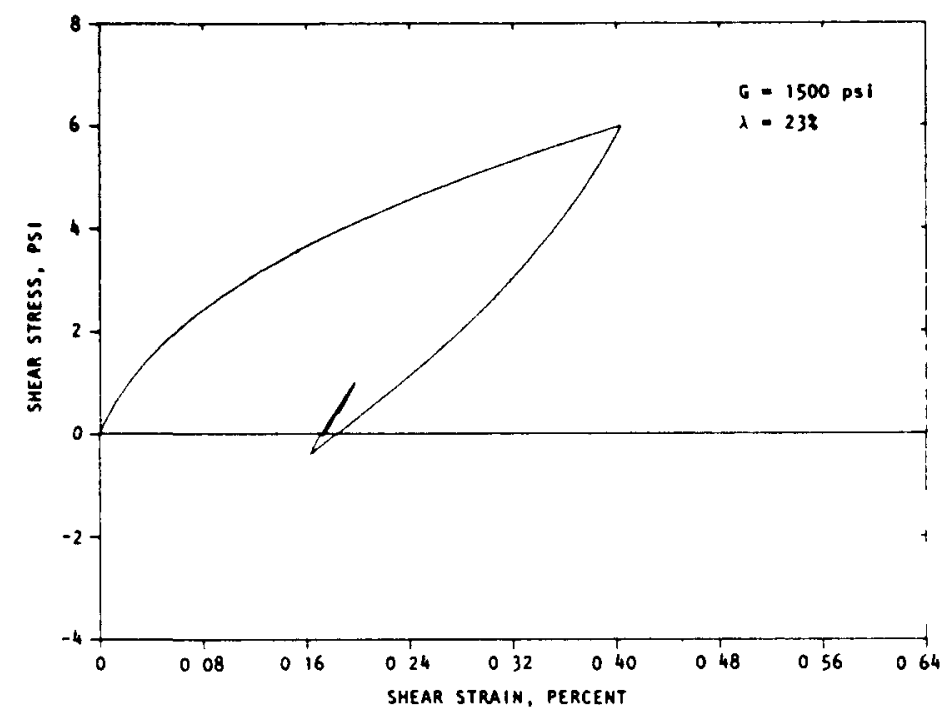

(a) Element $480,1 \mathrm{in.}$ from edge of anchor

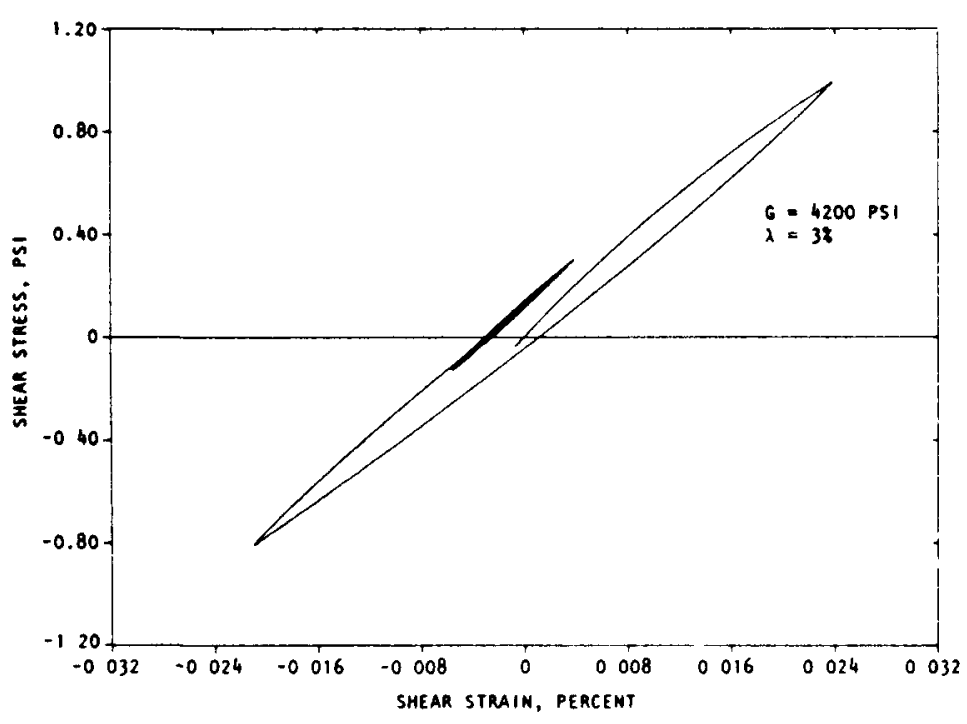

(c) Element $488,24 \mathrm{in.} \mathrm{from} \mathrm{edge} \mathrm{of} \mathrm{anchor}$

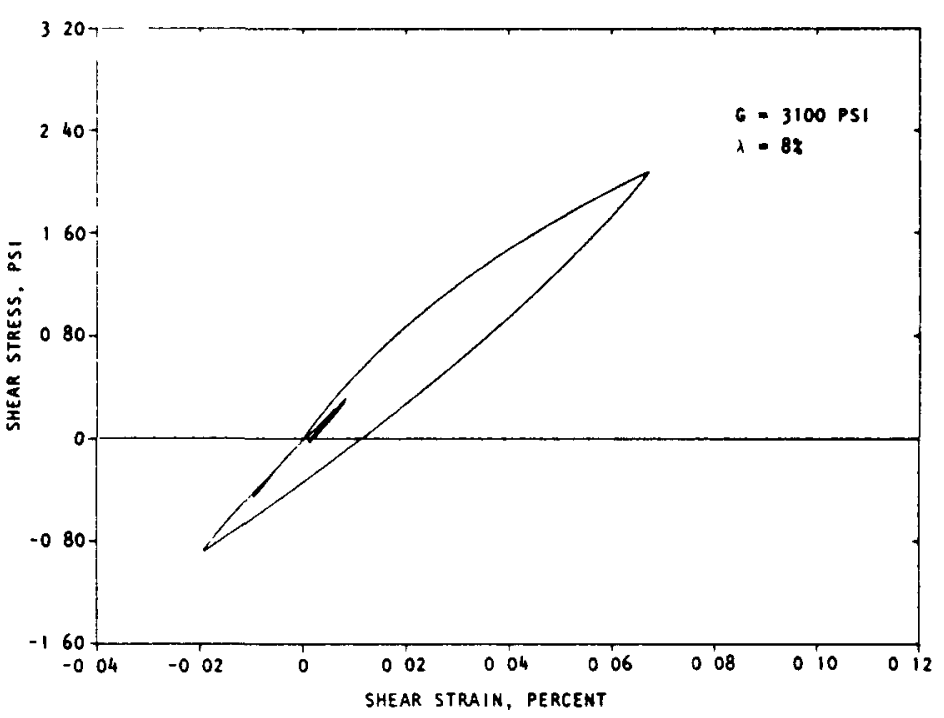

(b) Element $434,10.5 \mathrm{in}$. from edge of anchor

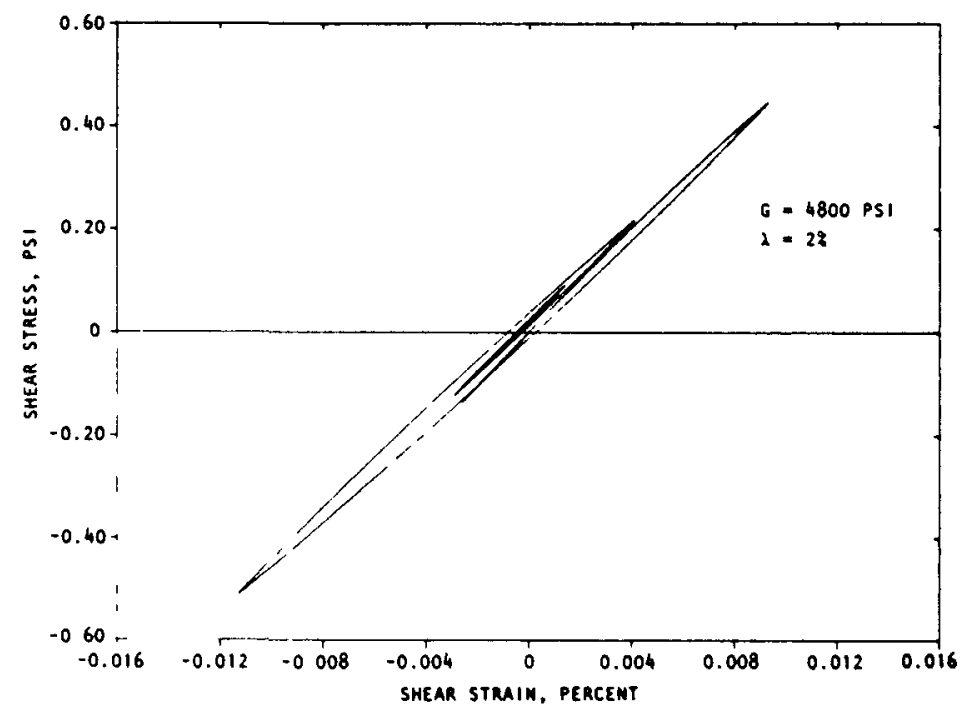

(d) Element $494,57 \mathrm{in}$. From edge of anchor

FIGURE 4-12. SHEAR STRESS VS. SHEAR STRAIII CURVES FOR SELECTED SOIL ELEMENTS -- NOIILINEAR FINITE ELEMENT RESULTS 
the anchor. The most rapid changes in both $G$ and $\lambda$ occur near the anchor. The changes in both parameters become much more gradual with increasing distance from the anchor.

Due to the hysteretic nature of the soil material, permanent distortions of elements near the anchor remain after the excitation has quieted down. This effect can be seen most clearly in Fig. 4-12a. These permanent strains can be explained by making the observation that equilibrium is a condition requiring zero shear stress. The return to equilibrium from the state of maximum stress follows a stress/strain path different from that by which maximum stress was originally attained; therefore, the strain at which zero stress prevails is nonvanishing. As seen in Fig. 4-12, this phenomenon quickly disappears with increasing distance from the anchor.

4.3.3 Assessment of Data Processing Procedures for Determination of In Situ shear wave Velocities

The shear wave velocity is an important property of the soil medium in that it leads directly to the shear modulus by way of the relationship

$$
\mathrm{G}=\rho \mathrm{V}_{\mathrm{s}}^{2}
$$

where $G$ is the shear modulus, $\rho$ is the mass density, and $V_{s}$ is the shear wave velocity, all expressed in a consistent set of units. In addition, the shear wave velocity is required for the estimation of shear strain, as discussed in section 4.3.4.

Thus far in the in situ test program, two different approaches have been used to estimate shear wave velocities. The first approach is termed the time-history method and identifies a particular feature within the measured velocity histories 
recorded at adjacent sensors. The delay time associated with that feature, i.e., differences in the time of occurrence of the feature at adjacent sensors, is determined from the sensor records. The shear wave velocity of the soil medium between these sensors is then computed as the ratio of the sensor spacing and the delay time.

A second approach is termed the waveform method and utilizes the displacements at the various sensors (obtained by integrating the velocity histories) to define wave profiles at different discrete times. A specific feature on each displacement profile, such as the crest of the wave, is then identified, and its location within the soil medium is noted. The distance traveled by this feature during a given time interval is used to estimate the shear wave velocity.

a. $\quad$ ime-History Methods

The time-history methods primarily used thus far for estimating shear wave velocities from in situ response measurelnents have been based on the first downward peak of the velocity history and the zero crossing of the velocity history after this peak (which corresponds to the first downward peak of the displacement history). These features were identified in the velocity histories for nodal points located along the middepth of the anchor. The resulting shear wave velocity computations were then compared to the original soil properties used as input to both the elastic and the nonlinear finite element calculations.

When computing shear wave velocities using timehistory methods the time required for a feature of the velocity signal to propagate a given distance is first noted. The shear wave velocity is then computed as the ratio of the given distance 
to this propagation time. Geometrically, this process amounts to computing the slope of the position-time diagram of the feature being observed. Thus, the computation of shear wave velocity involves a process of numerical differentiation, a process which is well known for its tendency to magnify the fluctuations of a time-history record. This tendency to exaggerate local fluctuations can be counteracted somewhat by choosing large distances or time intervals over which the differentiation is performed. However, this carries with it a penalty in sensitivity.

Fig. 4-13a shows the shear wave velocities as computed by the method of zero crossing of the velocity histories for the elastic case. Fig. 4-13b shows the shear wave velocities as calculated by the method of peak velocities. In both cases the actual shear wave velocity is indicated by a solid line. Minimum sensor spacings of 12 inches, 30 inches, and 60 inches have been used in these figures. The calculations involving 12 inches minimum spacing show an objectionable amount of scatter, while those corresponding to 30 inches and 60 inches are relatively scatter free. The results of the zero-crossing method agree remarkably well with the actual shear wave velocity. The results of the peak-velocity method are less accurate near the anchor, and improve with increasing distance from the anchor. However the results are acceptable throughout the entire radius range shown in the figure.

Fig. 4-14a shows the zero-crossing method applied to the results of the nonlinear calculation, while Fig. 4-14b shows the results of the peak-velocity method. The solid line in each figure shows the shear wave velocity based on the secant shear modulus of each element of soil. The secant shear modulus is computed as the peak. shcar stress divided by the peak shear strain of each element. For the 12-inch minimum spacing, there is an objectionable amount of scatter associated with the peak- 


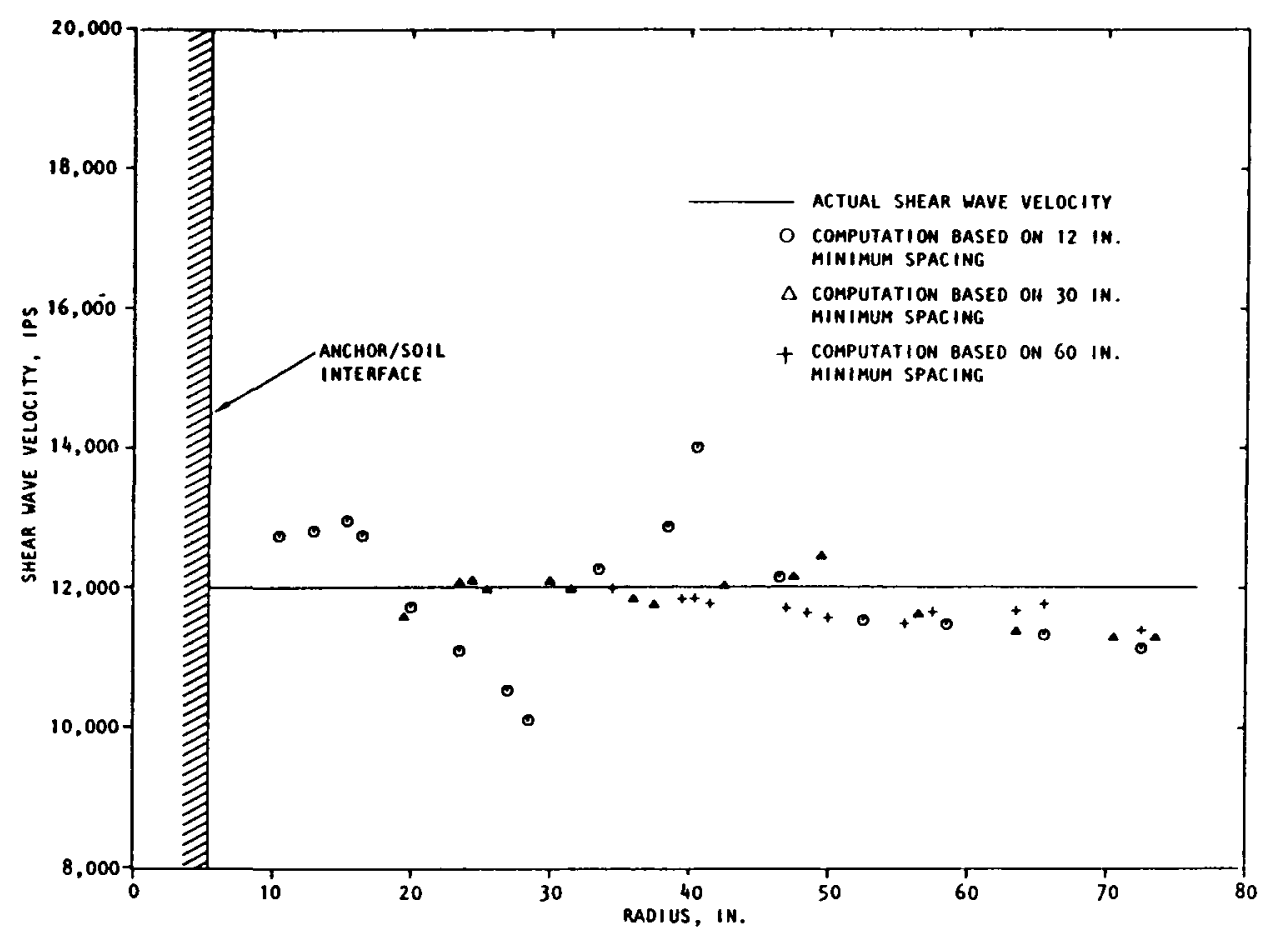

(a) Zero crossing of velocity history

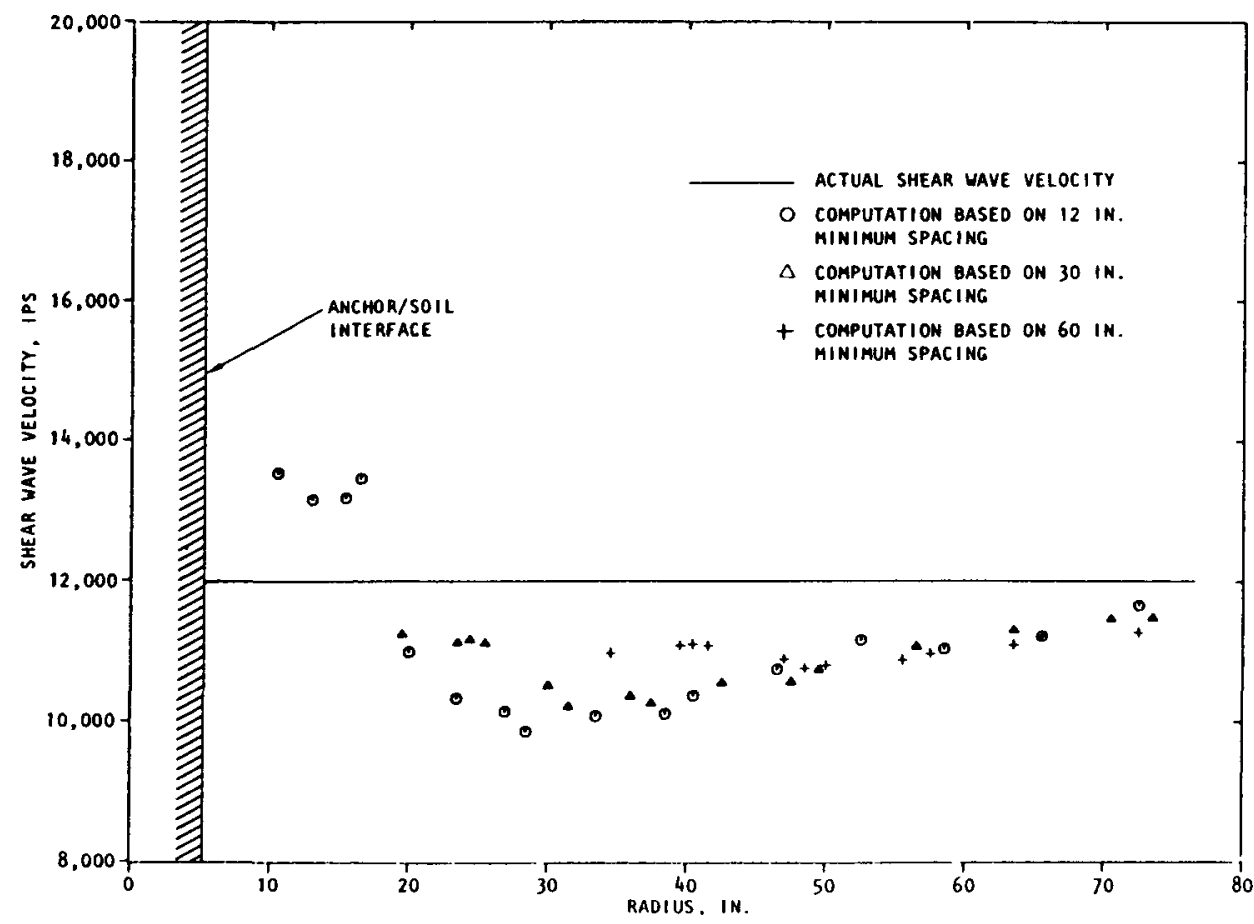

(b) First peak of velocity history

FIGURE 4-13. SHEAR WAVE VELOCITIES COMPUTED USING TIME-HISTORY METHODS -- ELASTIC FINITE ELEMENT RESULTS 


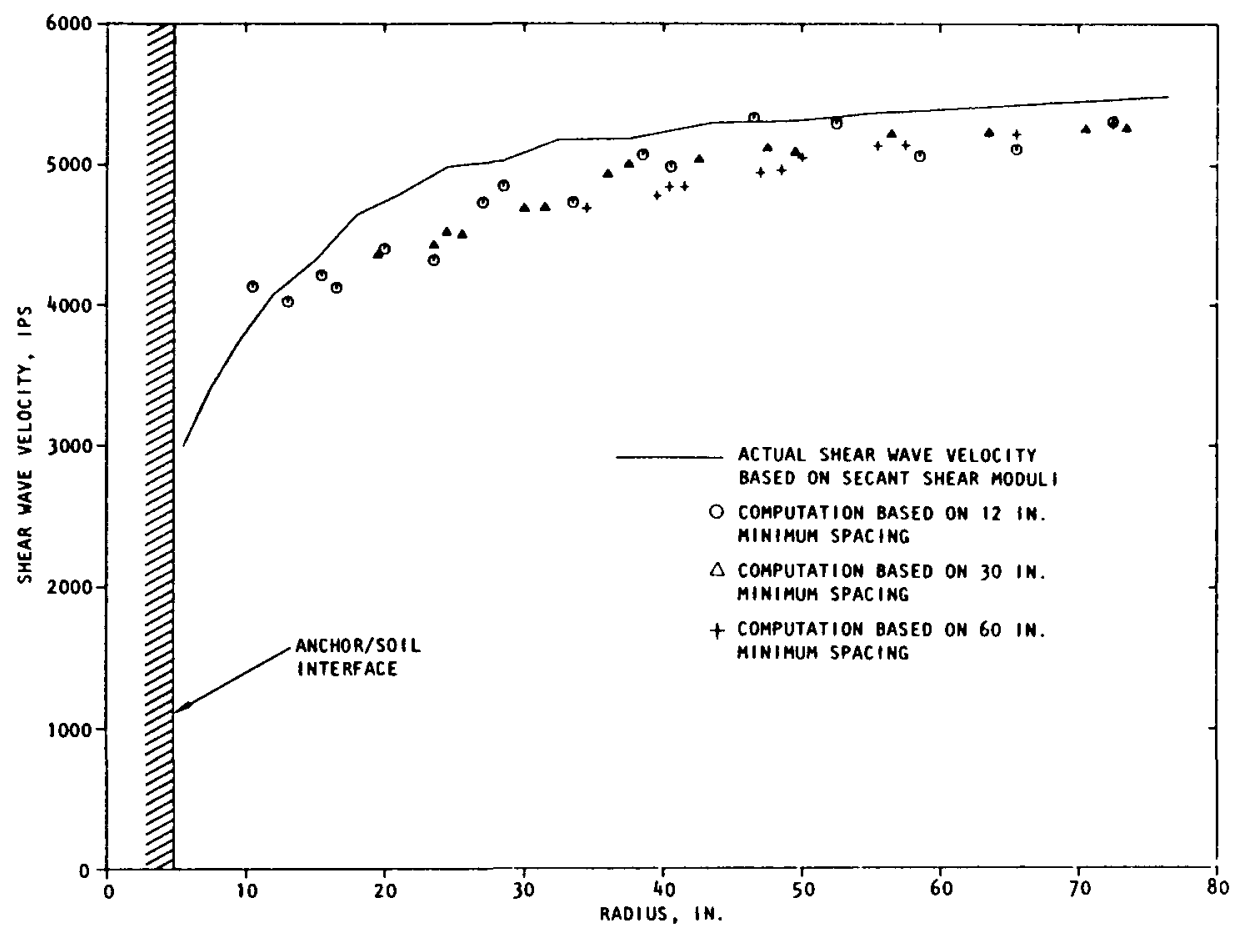

(a) Zero crossing of velocity history

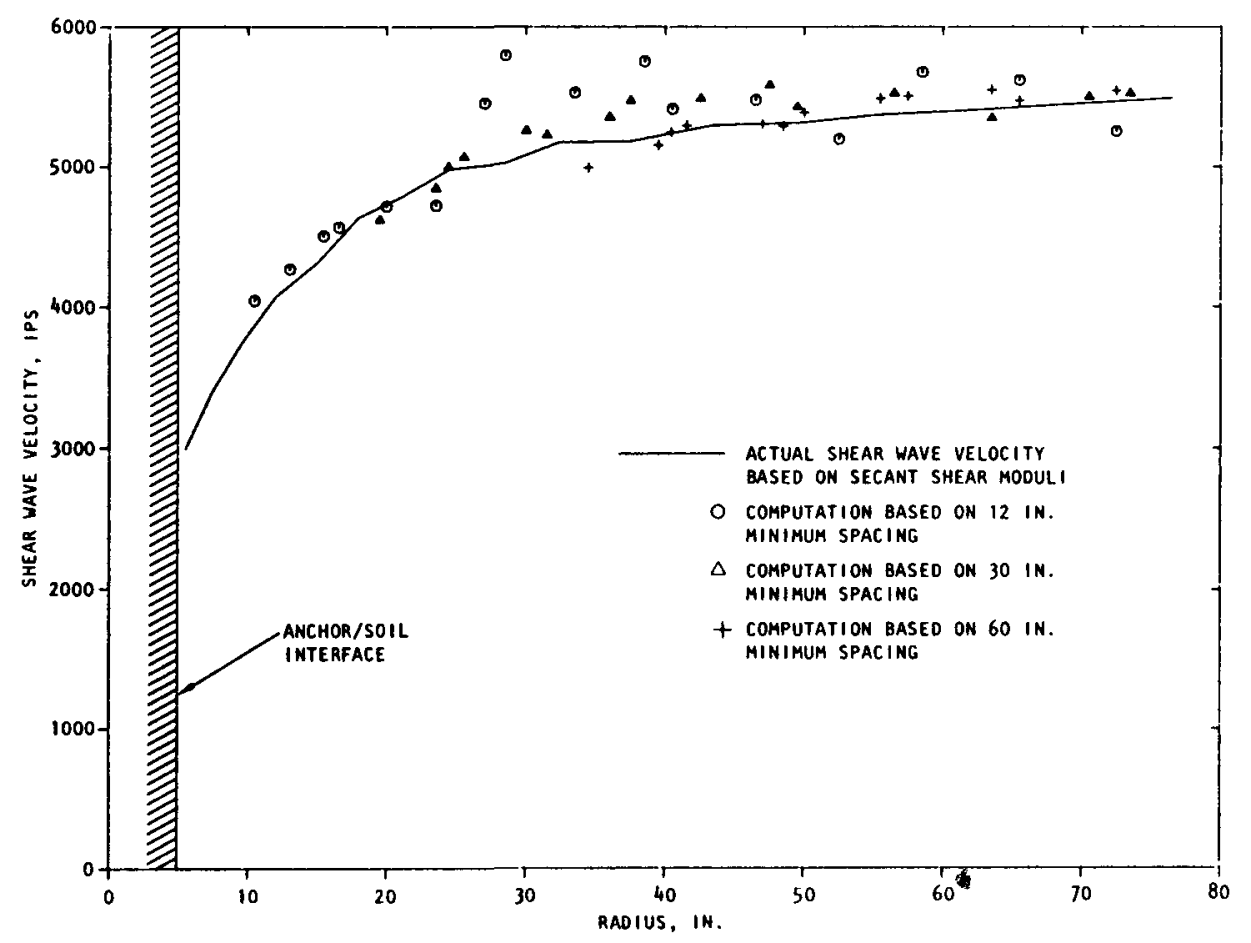

(b) First peak of velocity history

FIGURE 4-14. SHEAR WAVE VELOCITIES COMPUTED USING TIME-HISTORY METHODS -- HOHLINEAR FINITE ELEHENT RESULTS 
velocity method. In fact, the peak-velocity method seems to show more scatter at all sensor spacings than does the zerocrossing method. On the other hand, disregarding the results obtained using 12-inch minimum spacing, the peak-velocity method seems to fall slightly closer to the actual shear wave velocity. The difference between the results of the two methods may be related to the trend whereby the peak velocity occurs while the element is undergoing peak shear stress, whereas peak displacement occurs during unloading under conditions of low stress. Due to nonlinearity, therefore, the shear wave velocities involved in the propagation of the peak velocity and the zero crossing are slightly different. In the case of the nonlinear finite element calculation, the peak-velocity method produced slightly superior results. However, it cannot be concluded that this method will prove to be superior in all cases. In spite of the slight difference in results, both methods provide acceptable accuracy for the more distant stations (i.e., for distance greater than 20 inches from the anchor).

The above methods for determining shear wave velocity involve measurement of the travel time of some feature of the wave as it propagates from one sensor to another. The shear wave velocity computed in this manner is an average value for the region between the sensors. An alternative approach, referred to as the curve-fit approach, enables the determination of shear wave velocity at a point, rather than for a region between sensors. To apply this approach, time-of-occurrence data for the wave feature under observation are required at several locations. A curve of the form

$$
T(r)=\frac{a_{1}}{r}+a_{2}+a_{3} r
$$

is then fitted to these data, where $T(r)$ is the time-of-occurrence function, $r$ is the distance from the axis of symmetry, and $a_{1}$ ' $\mathrm{a}_{2}$, and $\mathrm{a}_{3}$ are undetermined coefficients. The slope of the curve 
at any point determines the shear wave velocity at that point. since this can be computed analytically, there is no numerical differentiation associated with this approach. Reduced scatter, therefore, is one of its advantages.

Fig. 4-15 shows time-of-occurrence data for zero crossing and peak velocity for nodal points along the middepth of the anchor in the nonlinear finite element calculation. These data have been fitted, using a least-squares procedure, with curves having the form of Eq. 4.2. These curves are shown in the figure as solid lines. It is seen that the comparison between the analytical approximation of $\mathrm{Eq} \cdot 4.2$ and the raw data is excellent. It can be concluded, therefore, that Eq. 4.2 is an appropriate form with which to seek an analytical approximation of the time-of-occurrence data. Eq. 4.2 can be used to fit these data as long as there are at least three data points.

Fig. 4-16 shows the shear wave velocity of the nonlinear finite element calculation as computed by the zerocrossing and peak-velocity curve-fit methods. The time-ofoccurrence functions, Eq. 4.2, were fitted based on data from all of the nodal points at the middepth of the anchor. Shear wave velocity was then computed at each nodal point location. The resulting data sets show no scatter at all. In fact, since the time-of-occurrence function is represented analytically, it would have been possible to plot the shear wave velocity calculations as continuous curves. It is evident from Fig. 4-16 that shear wave velocity measurements at the anchor-soil interface are possible, using the curve-fit approach. This was not the case using the original approaches for estimating travel times. Furthermore, the accuracy seems to be acceptable throughout the range of Fig. 4-16 for both methods. Had fewer points been available for fitting the time-of-occurrence function, a slightly different analytical fit would, most likely, have been obtained. However, the results would have been equally free of 


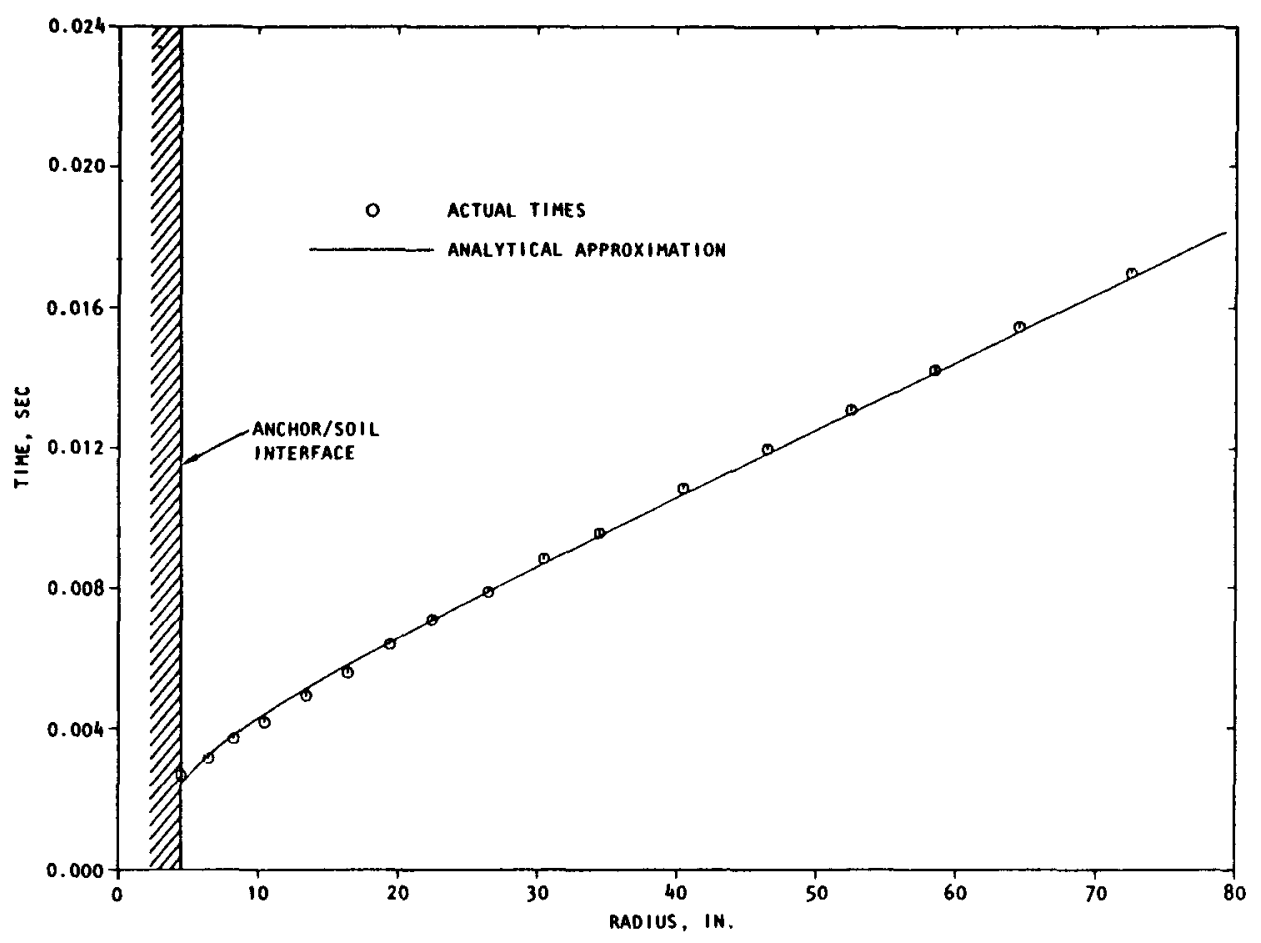

(a) Arrival times of zero crossing of velocity

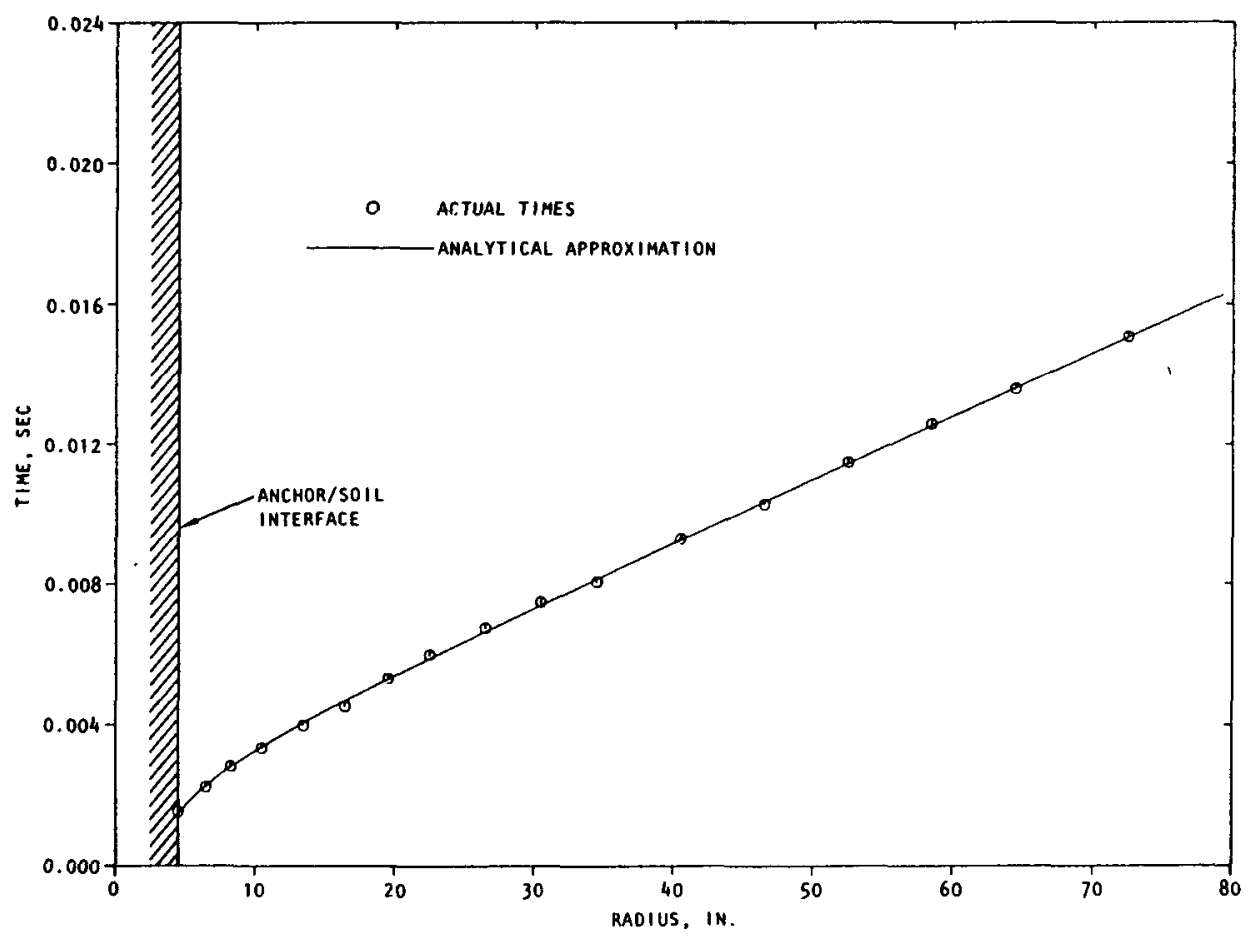

(b) Arrival times of peak velocity

FIGURE 4-15. TIMES OF PEAK AND ZERO CROSSING OF COMPUTED VELOCITY HISTORIES -- NONLINEAR FINITE ELEMENT RESULTS 


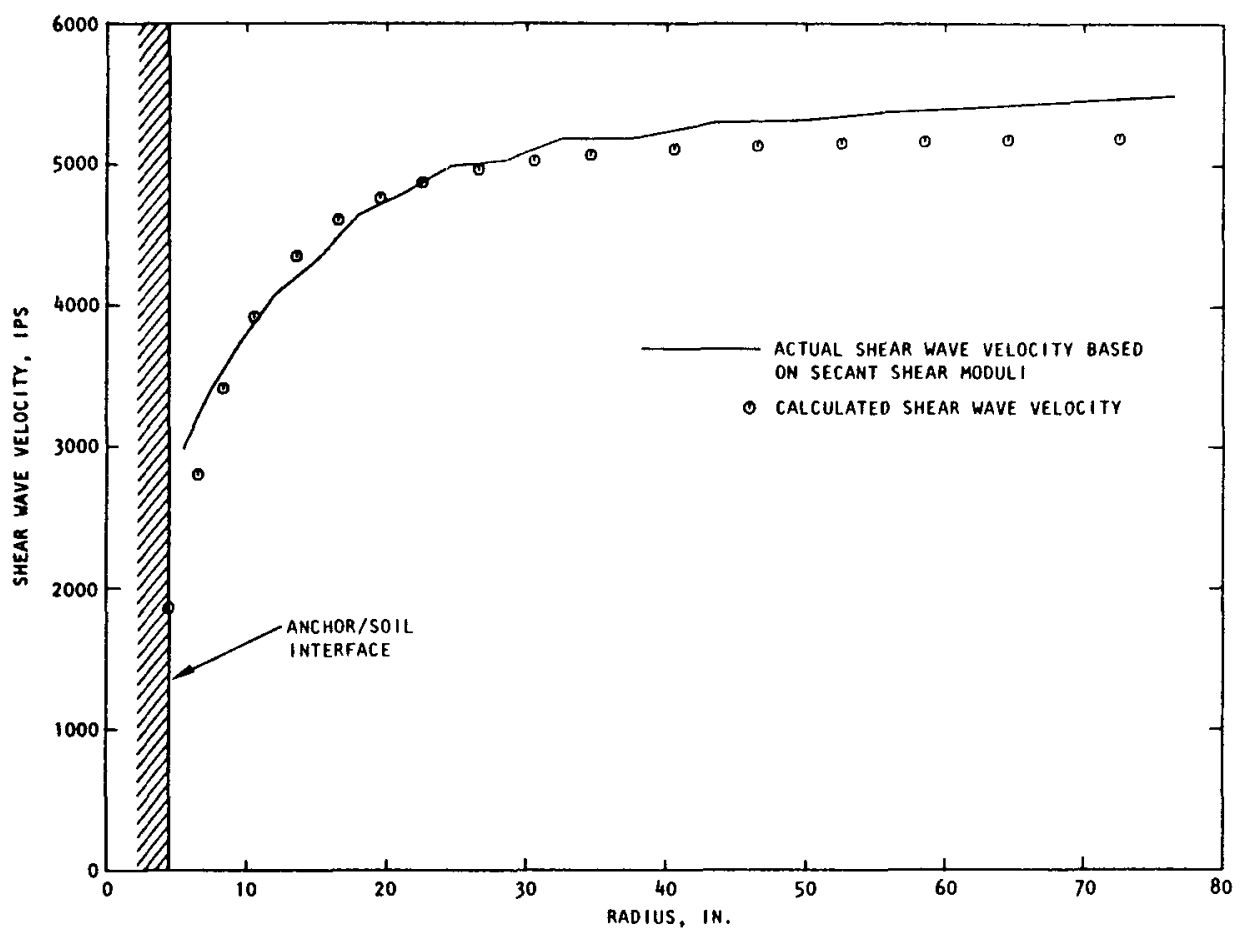

(a) Zero-crossing curve-fit method

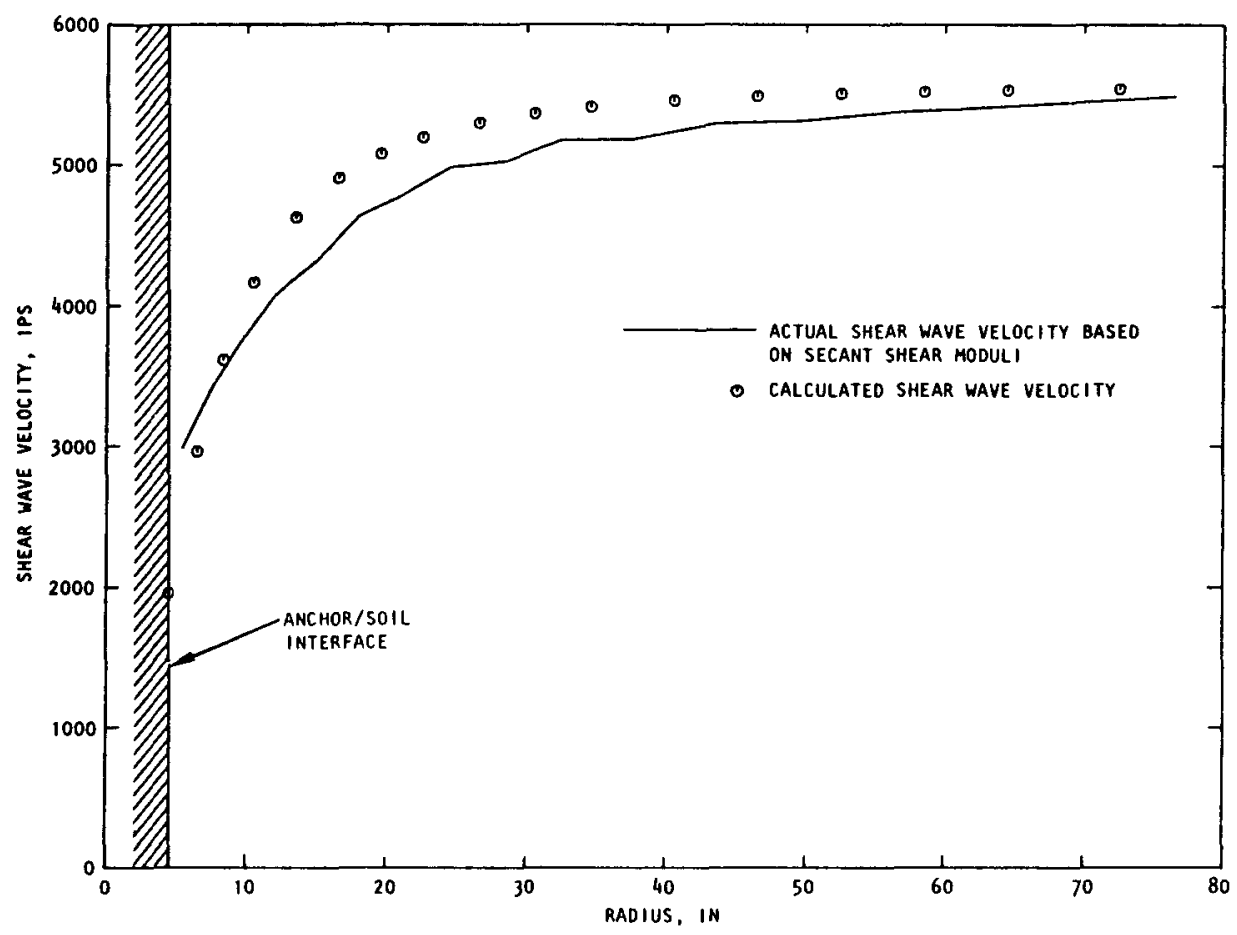

(b) Peak-velocity curve-fit method

FIGURE 4-16. SHEAR WAVE VELOCITIES COMPUTED USING CURVE-FIT APPROACH -NONLINEAR FINITE ELEMENT RESULTS 
scatter and the amount of error would probably not have been much greater.

\section{b. Waveform Methods}

The time-history methods of computing shear wave velocity in the soil medium are based on the propagation of distinctive features of the response history observed at isolated points in the soil medium. The waveform methods, on the other hand, are based on the propagation of distinctive features of the spatial distribution of free-field response observed at isolated instants of time. The waveform methods employed in the in situ test program thus far have been based primarily on observations of the crest of the displacement wave. Although, in theory, the shear wave velocity can be determined through the observation of any feature of the wave, the displacement crest has been selected primarily because it is easily identified and relatively simple to work with. Furthermore, it has the physical significance that it is a point of zero shear strain and peak displacement, and occurs as the first identifiable point on the velocity history measurement after the peak strain has occurred. Thus it is more than merely an identifiable label on the response profile; it is a significant feature of the propagating shear disturbance.

Application of the waveform methods to in situ data is hampered by a shortage of sensors in typical production tests. The spatial resolution of velocity gages ordinarily used in in situ production tests is inadequate for accurately constructing the response profile at a given instant of time. On the other hand, application of waveform methods to the finite element results, due to the abundance of nodal points distributed along the midplane of the anchor, does not suffer due to inadequate spatial resolution. Several displacement profiles, constructed from the elastic finite element results, are shown 
in Fig. 4-17a. The propagation of the crest of the displacement profiles is an easily recognized feature of this figure. The envelope of peak displacements is also shown in Fig. 4-17a. The peak displacement at a given location occurs when the displacement profile is tangent to the peak-displacement envelope at that point. It is obvious from the figure that when this occurs, the crest of the displacement profile occupies a different position, closer to the anchor than the point which is undergoing its peak displacement. Thus, at points close to the anchor, the peak displacement at a point and the crest of the displacement profile at that point do not correspond to the same event. This is the basic difference between the timehistory methods and the wave-form methods. However, beyond several feet from the anchor, the rate of attenuation of displacement with distance is smal1, and the crest of the wave-forms is identical to the zero-crossing point in the time-history method.

Fig. 4-17b shows several displacement profiles constructed from the nonlinear finite element results. The permanent displacements of near-anchor soil elements, which were discussed in Section 4.3.1, are evident in these profiles. These permanent displacements are of such magnitude that the displacement of a given nodal point near the anchor is never exceeded by the displacement of any other nodal points near the anchor at which the crest of the displacement profile never occurs. Even at several nodal points where there is a local maximum of the displacement profile, this feature is indistinct, making it difficult to determine the precise location of the crest. These problems render the wave-form methods ineffective in determining the shear wave velocity for the nonlinear case, particularly near the anchor. Although this phenomenon of permanent displacements near the anchor was not observed experimentally, it was pointed out in section 4.3 .1 that permanent displacements probably were present. 


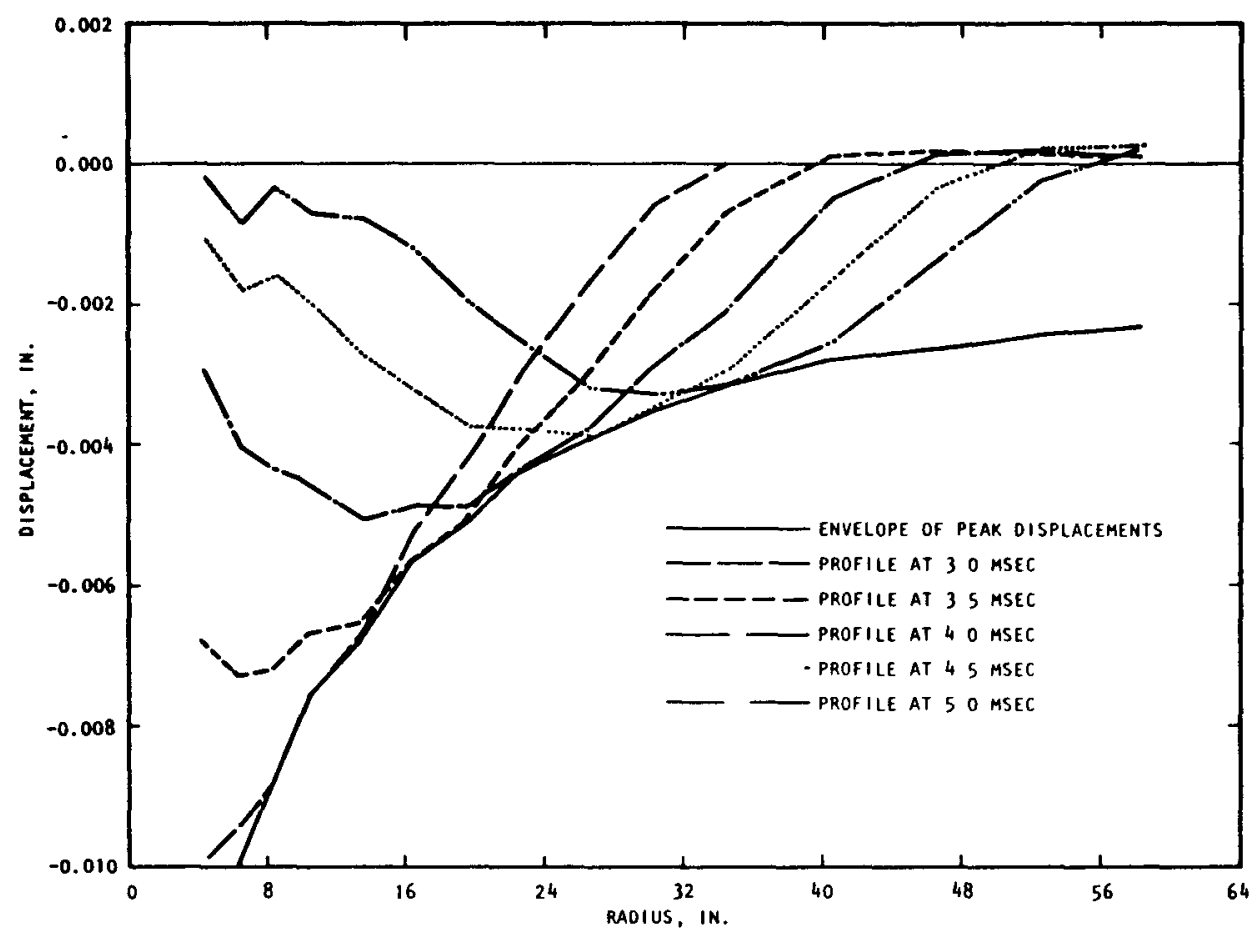

(a) Elastic finite element results

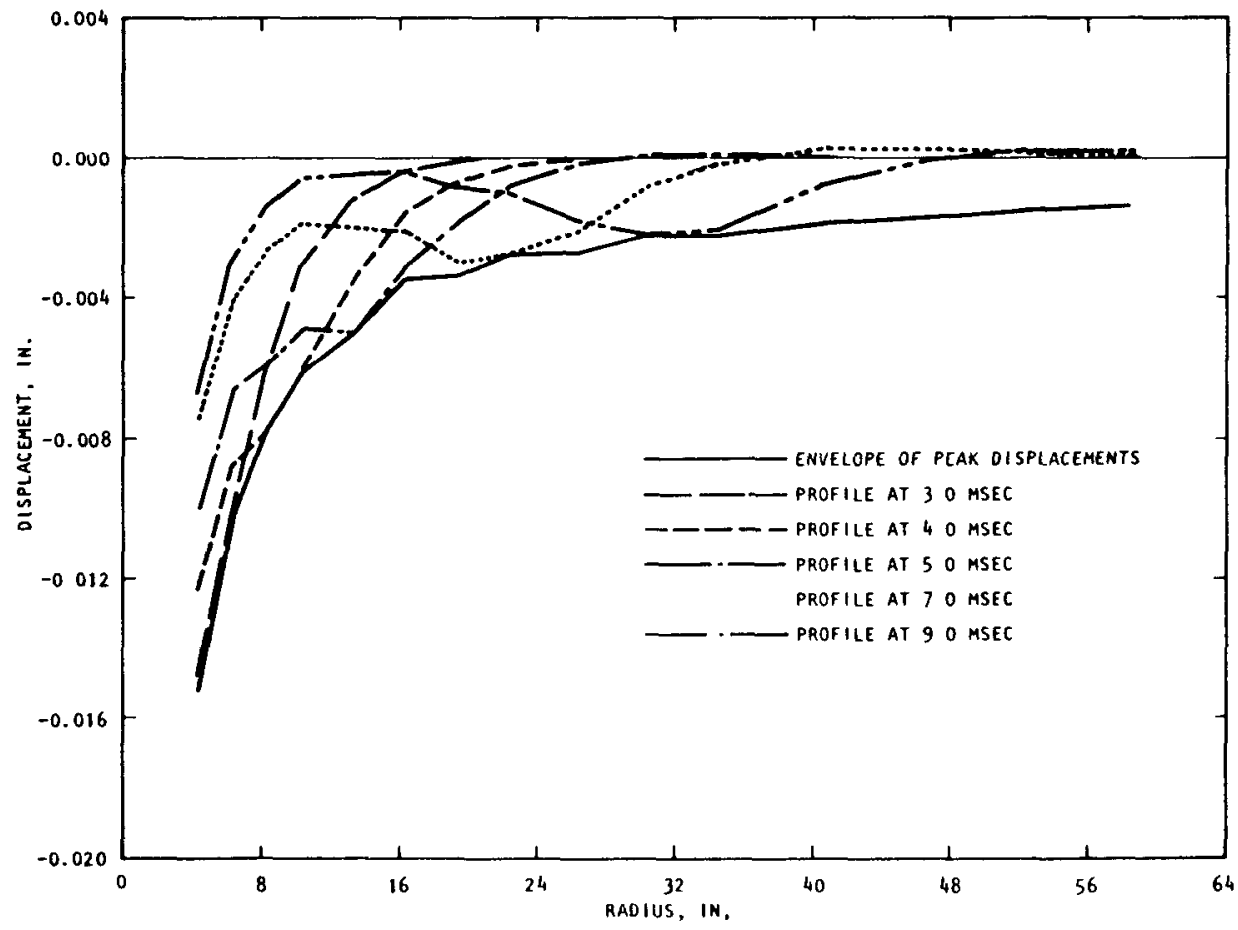

(b) Nonlinear finite element results

FIGURE 4-17. DISPLACEMENT PROFILES AT VARIOUS INSTAINTS OF TIME ALONG THE MIDDEPTH OF THE ANCHOR 


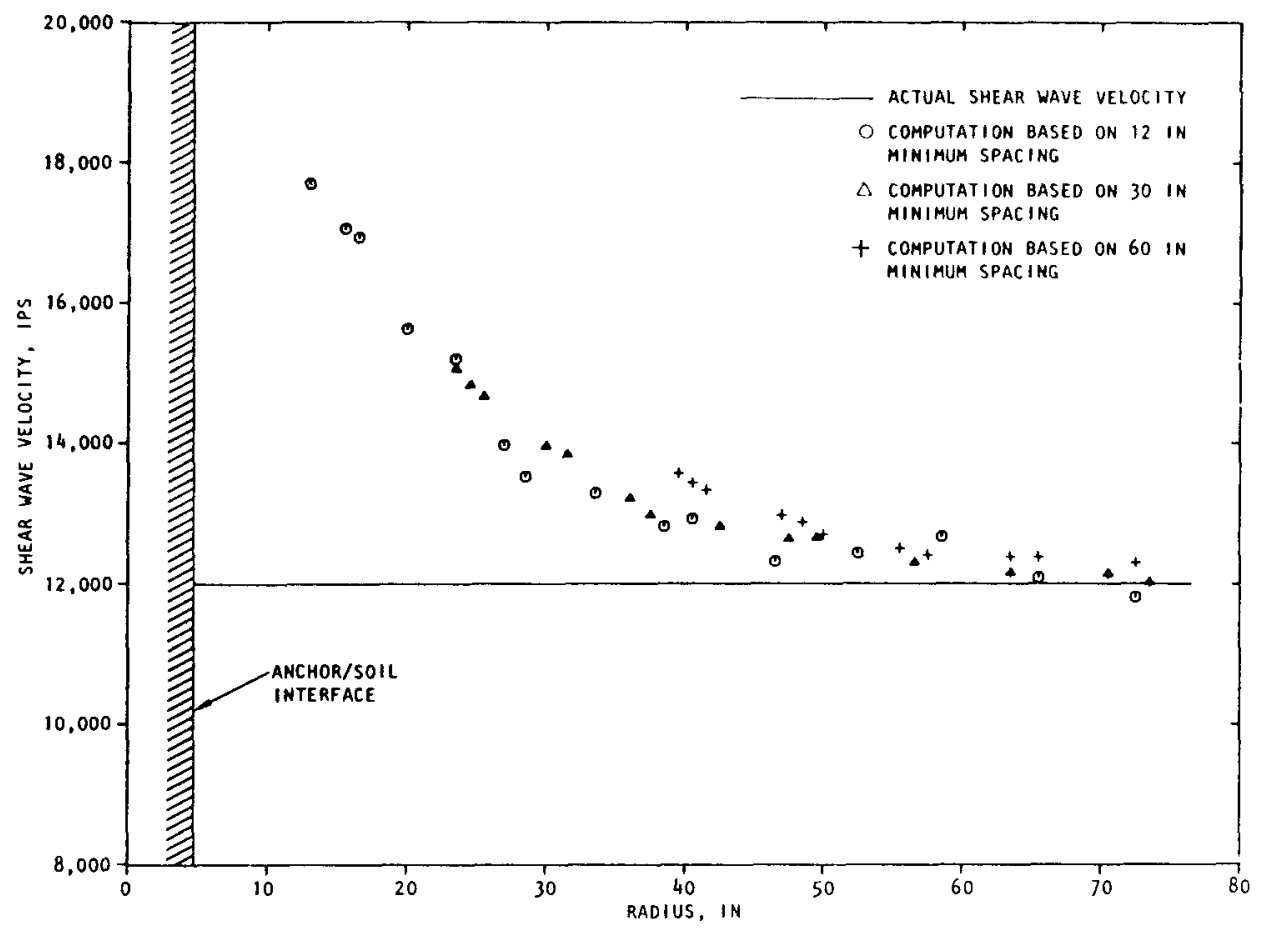

FIGURE 4-18. SHEAR WAVE VELOCITIES COMPUTED USIHG WAVE FORM METHODS -ELASTIC FINITE ELEMENT CALCULATION 
The displacement profiles of the elastic finite element calculation can be used to determine the position of the crest of the displacement wave at any given instant of time. In order to improve the precision with which the position of the wave crest can be determined, a parabolic interpolation scheme has been used. Using this method, the displacements at several points in the vicinity of the crest of the profile are represented analytically using a parabola in which the coefficients are chosen by a least-squares procedure. The crest of the profile is taken to the point at which the parabola has zero slope.

Results of this procedure are presented in Fig. 4-18 in which the sensor spacings of 12 inches, 30 inches, and 60 inches have again been used. The lack of scatter, even for the 12 inch sensor spacing, as well as the fact that crest locations obtained by parabolic interpolation are consistent with profile plots, indicates that the degree of precision with which this method has been applied must have been high. An obvious trend whereby excessively high wave speeds are recorded near the anchor suggests that the crest of the displacement profile propagates at a velocity different from the shear wave velocity near the anchor. This observation implies that waveform methods based on the crest of the displacement profile are not well suited for determining shear wave velocities in the near-anchor region. As a result, it is recommended that subsequent data reduction be performed using time-history methods, and that the wave-form methods be dropped from further consideration for computing shear wave velocities.

4.3.4 Assessment of Data Processing Procedures for Determination of In Situ Shear Strain

The present approach used for estimating shear strains from the in situ response measurements is based on an analogy with the propagation of plane shear waves in an elastic medium 
of infinite extent. The resulting formula is:

$$
\gamma_{r z}=\frac{\dot{w}}{\bar{V}_{s}}
$$

where: $\gamma_{r z}$ shear strain at the point under consideration

$\dot{\mathrm{w}}$ particle velocity at the point under consideration

$\mathrm{V}_{\mathrm{s}}$ shear wave velocity of the soil medium at the point under consideration

A discussion of the validity of Eq. 4.3 is given in Chapter 3. This discussion indicates that Eq. 4.3 can be expected to yield accurate results at large distances from the anchor; however, near the anchor, the soil behavior becomes more complex and Eq. 4.3 no longer applies.

The implementation of Eq. 4.3 first requires an estimate of the shear wave velocity of the soil medium. Procedures for estimating this parameter have been described and assessed in section 4.3.3. Once the shear wave velocity has been obtained for a given location, the peak shear strain at that location can be determined by using the peak particle velocity in Eq. 4.3. The results are likely to vary slightly, depending on the particular method used to estimate the shear wave velocity. Fig. 4-19 shows comparisons between actual peak shear strains obtained in the finite element calculations and peak shear strains computed by using the peak-velocity curve-fit method in conjunction with Eq. 4.3. Fig. 4-19a shows this comparison for the elastic finite element calculation, while Fig. 4-19b shows the results of the nonlinear finite element calculation. As previously indicated in Chapter 3, good agreement has been obtained away from the anchor (in general, 20 to 25 inches away). Near the anchor, the agreement is not quite as good. 


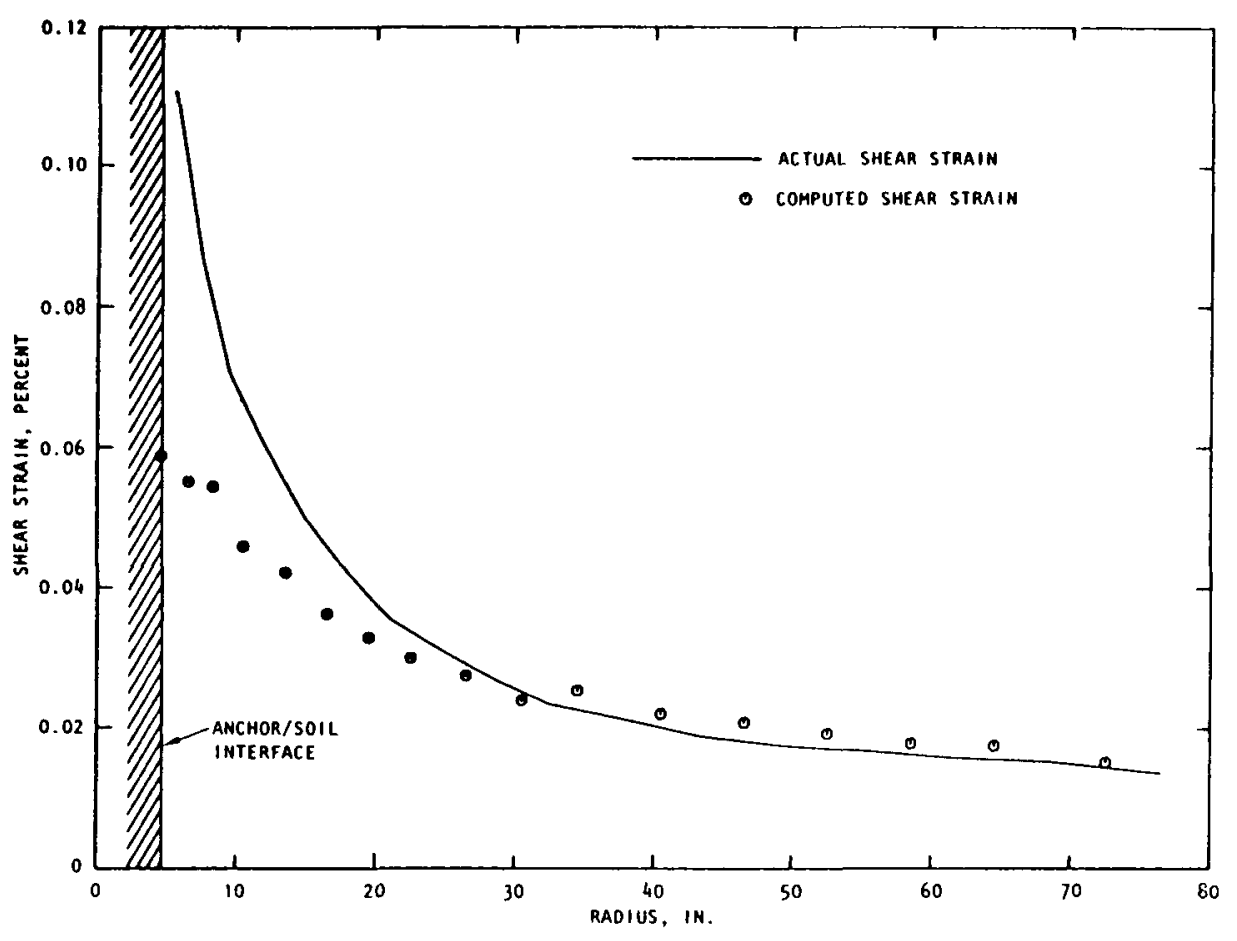

(a) Elastic case

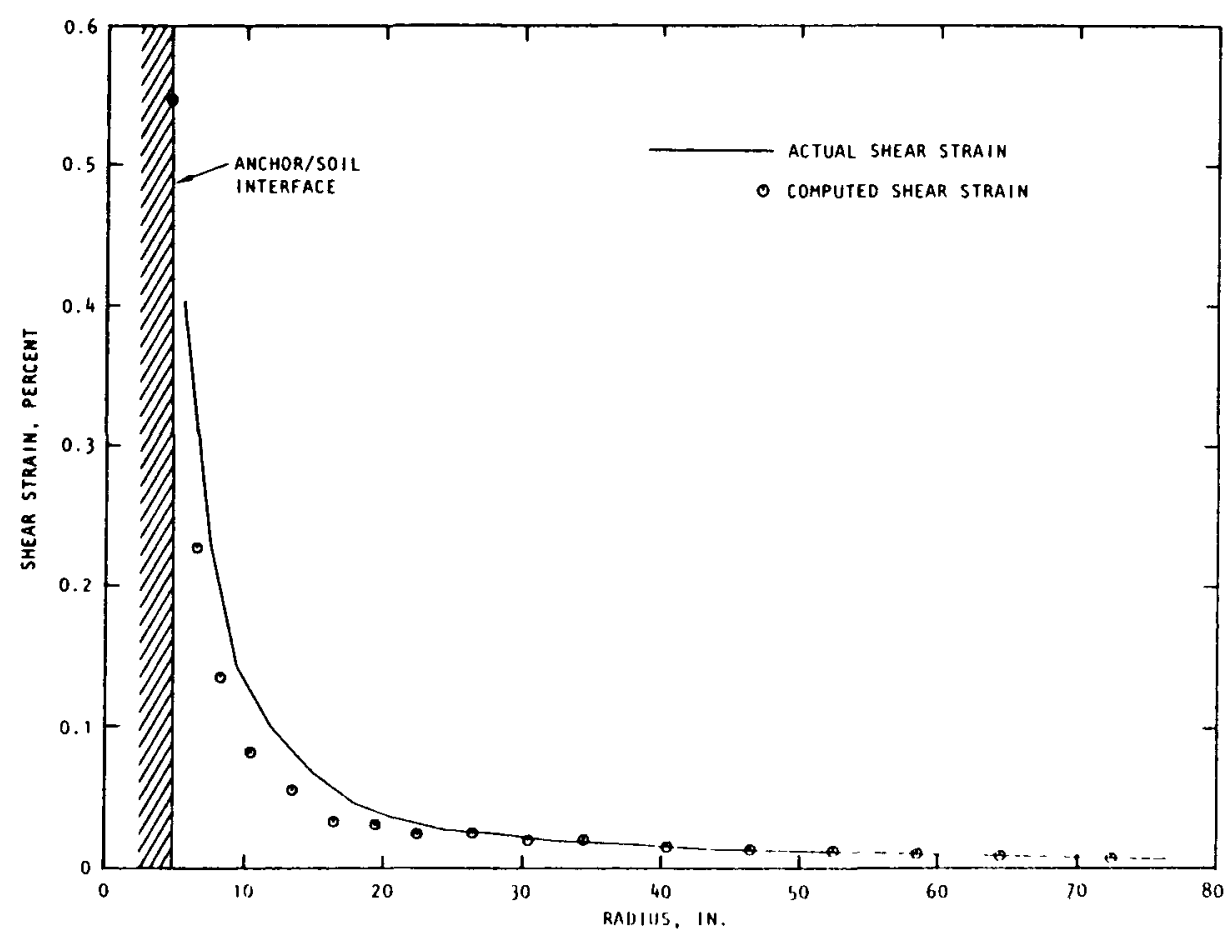

(b) Nonlinear case

FIGURE 4-19. COMPARISON OF ACTUAL PEAK STRAINS FROM THE FINITE ELEMENT CALCULATIONS WITH PEAK STRAIINS COMPUTED USING EQUATION $4-3$ 
When the shear wave velocity has been estimated using a method involving the travel time between sensors, the resulting value is an average value for the region between the sensors. The shear strain associated with this average value of shear wave velocity must also be an average value for the region. Three methods of computing an average shear strain for the region between two sensors are studied below. The test as to which of these methods is most compatible with the value of shear wave velocity obtained is considered in section 4.3 .5 .

The first proposed method of computing the average shear strain in the interval between two sensors is to estimate the shear strain at the midpoint between the two sensors. This method is referred to as the midpoint method. In order to estimate the shear strain at the midpoint of the region, the approximate distribution of shear strain between the sensors must be known. Fig. 4-20, which shows the distribution of peak shear strain obtained from the nonlinear finite element calculation, indicates that the relationship between peak shear strain and radius is nearly linear on a log/log grid. This suggests that the shear strain distribution between two sensors can be approximated by the expression:

$$
\gamma_{r z}=\operatorname{Ar}^{\alpha}
$$

$$
\text { where: } \begin{aligned}
\gamma_{\text {rz }} & \text { shear strain } \\
r & \text { distance from axis of symmetry } \\
\mathrm{A}, \alpha & \text { arbitrary parameters }
\end{aligned}
$$

The shear wave velocity for the region between the sensors, and the peak particle velocity at each sensor can be used to estimate the peak shear strains at the two sensors. By enforcing the conditions that Eq. 4.4 should assume these values of strain when $r$ equals the coordinates of the sensors, the values of $A$ and $\alpha$ for the interval between the sensors can be computed. By 


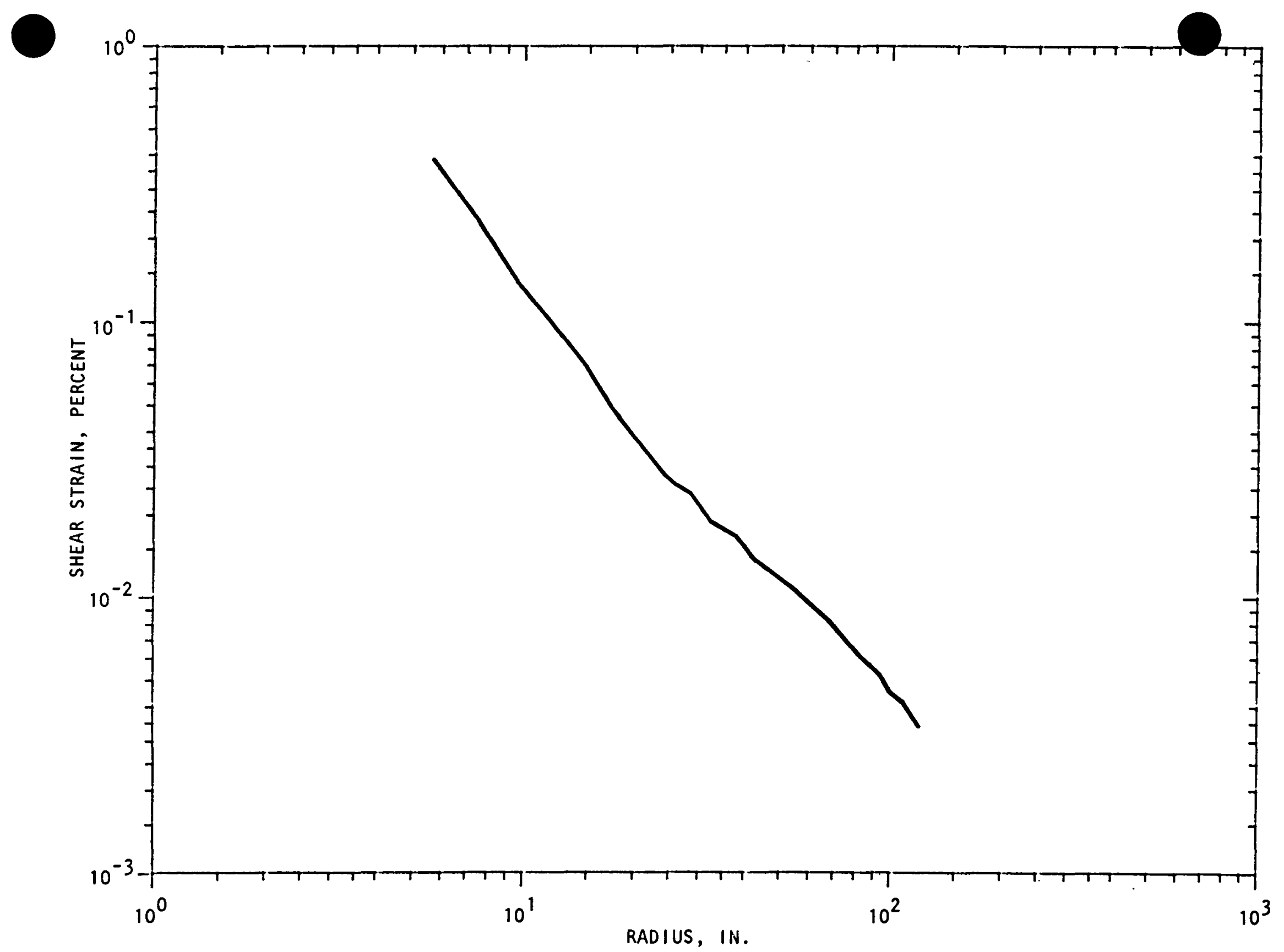

FIGURE 4-20. PEAK SHEAR STRAIN VS. DISTANCE FROM THE AXIS OF SYMPETRY -- NONLIINEAR FINITE ELEMENT RESULTS 
setting $r$ equal to the coordinate of the midpoint between the sensors, Eq. 4.4 gives the average strain determined by the midpoint method.

The second method of computing the average shear strain for the region between two sensors is referred to as the integration method. In this method, the distribution of shear strain between the two sensors is represented by Eq. 4.4, in which $A$ and $\alpha$ are determined in the same manner as was done in the case of the midpoint method. Eq. 4.4 is then integrated with respect to $r$, with limits corresponding to the coordinates of the two sensors involved. The result of this integration is then divided by the distance between the sensors to obtain the integrated average shear strain.

The third method of estimating the average shear strain for the region between the sensors is referred to as the endaverage method. In this method, the shear strains at the two sensors are estimated as was done in the case of the midpoint method. The straight numerical average of these two values is used as the average shear strain.

These methods of estimating shear strain, which are associated with the travel-time approach to estimating shear wave velocity, do not have as wide a range as the method used in Fig. 4-19. The region nearest the anchor escapes evaluation using these methods. The accuracy and range obtainable using these methods are considered in section 4.3.5.

\subsubsection{Evaluation of Total Data Reduction Systems}

Several methods of computing shear wave velocity from in situ data have been investigated in section 4.3.3. All of the time-history methods, including those which involve analytical curve fits as well as those which involve travel time between 
sensors, have survived the initial evaluation. The wave-form methods have been eliminated from further consideration because of practical limitations in working with this data and because of the apparent limitations of these methods in evaluating nearanchor soil responses (Section 4.3.3). In addition, several variations of the manner in which shear strains can be estimated, related particularly to the travel-time methods, have been proposed. These various methods for computing shear wave velocity and shear strain must be combined into total data reduction systems, and evaluated as such. The varıous combinations under consideration are identified in Table 4-1. The nonlinear finite element calculation is used to evaluate each of these procedures, based on the accuracy within which each procedure can reproduce the original shear modulus vs. shear strain relationship used as input to the finite element analysis. The shear modulus is obtained from the shear wave velocity using Eq. 4.1.

Fig. 4-21 shows the midpoint method for averaging shear strains used in conjunction with each of the travel-time methods for computing shear wave velocity to construct modulus/ strain relationships. Fig. 4-22 shows the integration method, while Fig. 4-23 shows the end-average method used along with the travel-time methods to compute modulus/strain relationships. In each of the figures, the relationships between secant shear modulus and shear strain actually obtained from the nonlinear finite element calculation is shown as the solid line. Minimum sensor spacings of 12 inches, 30 inches, and 60 inches were used in obtaining the computed modulus/strain relationships.

In each of the figures, the zero-crossing method for computing shear wave velocity resulted in less scatter than the peak-velocity method; however the results were less accurate. This may be due to the fact that peak velocity occurs when the shear stress is at a maximum, while the zero crossing does not. The end-average method for estimating strain is seen to be badly 
TABLE 4-1. KEY TO DATA REDUCTION PROCEDURES FOR DEVELOPMENT OF SHEAR MODULUS VS. SHEAR STRAIN RELATIONSHIPS

\begin{tabular}{|c|c|c|c|c|c|}
\hline \multirow{2}{*}{\multicolumn{2}{|c|}{$\begin{array}{l}\text { Method of Calculating } \\
\text { Shear Wave Velocity* }\end{array}$}} & \multicolumn{4}{|c|}{ Method of Calculating Shear Strain } \\
\hline & & $\begin{array}{l}\text { Analytical } \\
\text { Curve-Fit }\end{array}$ & $\begin{array}{l}\text { Midpoint } \\
\text { Method }\end{array}$ & $\begin{array}{c}\text { Integration } \\
\text { Method }\end{array}$ & $\begin{array}{c}\text { End-Average } \\
\text { Method }\end{array}$ \\
\hline $\begin{array}{l}\text { Analytical } \\
\text { Curve-Fit }\end{array}$ & $\begin{array}{l}\text { Peak } \\
\text { Velocity }\end{array}$ & $\begin{array}{l}\text { Peak-Velocityl } \\
\text { Fit Method }\end{array}$ & & & \\
\hline & $\begin{array}{l}\text { Zero } \\
\text { Crossing } \\
\text { of Velocity }\end{array}$ & $\begin{array}{l}\text { Zero-Crossing/ } \\
\text { Fit Method }\end{array}$ & & & \\
\hline $\begin{array}{l}\text { Travel Time } \\
\text { Between Sensors }\end{array}$ & $\begin{array}{l}\text { Peak } \\
\text { Velocity }\end{array}$ & ( & $\begin{array}{l}\text { Peak-Velocity/ } \\
\text { Midpoint } \\
\text { Method }\end{array}$ & $\begin{array}{l}\text { Peak-Velocity/ } \\
\text { Integration } \\
\text { Method }\end{array}$ & $\begin{array}{l}\text { Peak-Velocity/ } \\
\text { End-Average } \\
\text { Method }\end{array}$ \\
\hline & $\begin{array}{l}\text { Zero } \\
\text { Crossing } \\
\text { of Velocity }\end{array}$ & D & $\begin{array}{l}\text { Zero-Crossing/ } \\
\text { Midpoint } \\
\text { Method }\end{array}$ & $\begin{array}{l}\text { Zero-Crossing/ } \\
\text { Integration } \\
\text { Method }\end{array}$ & $\begin{array}{l}\text { Zero-Crossing/ } \\
\text { End-Average } \\
\text { Method }\end{array}$ \\
\hline
\end{tabular}

* Shear modulus is obtained from shear wave velocity using Equation 4-1. 


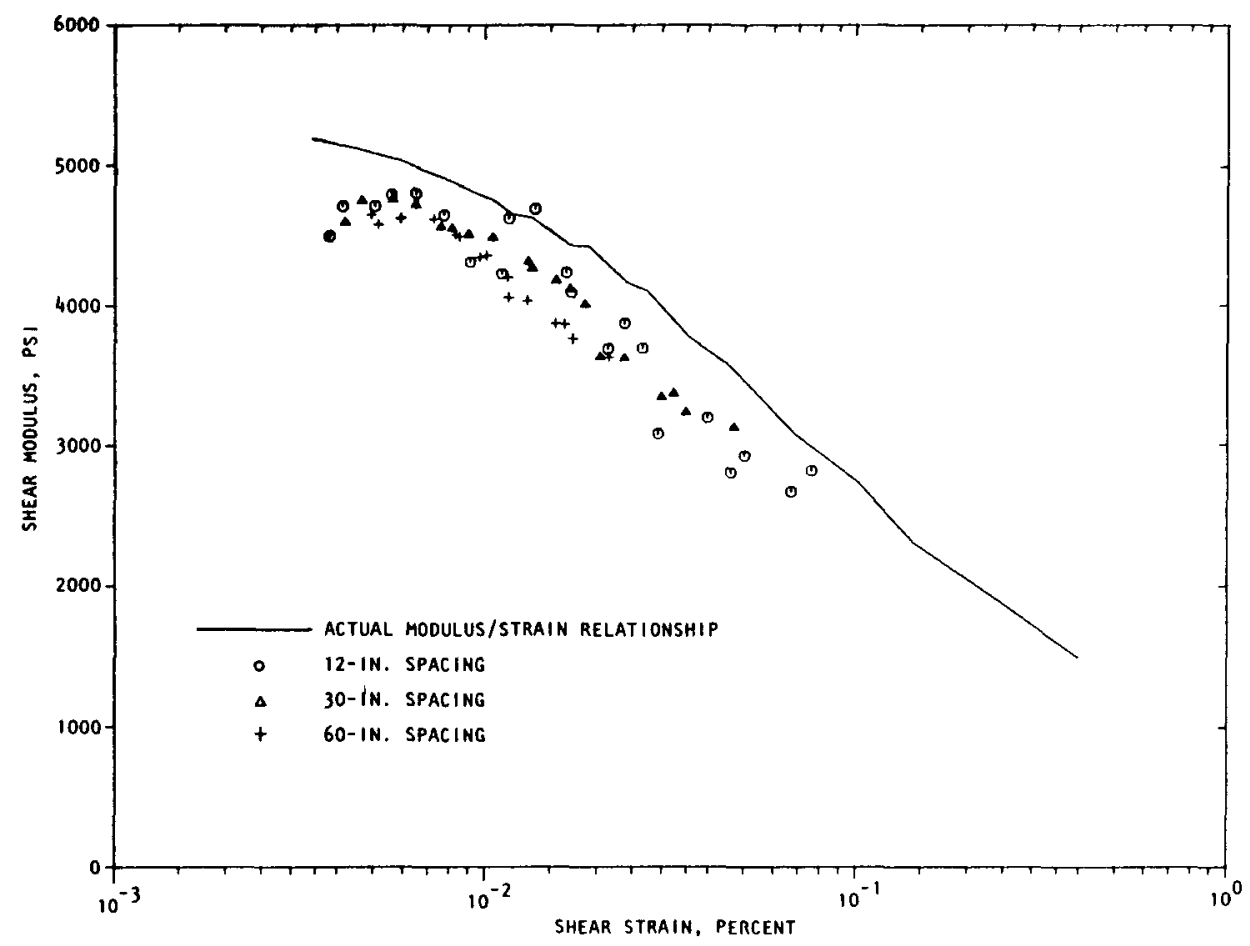

(a) Zero-crossing/midpoint method

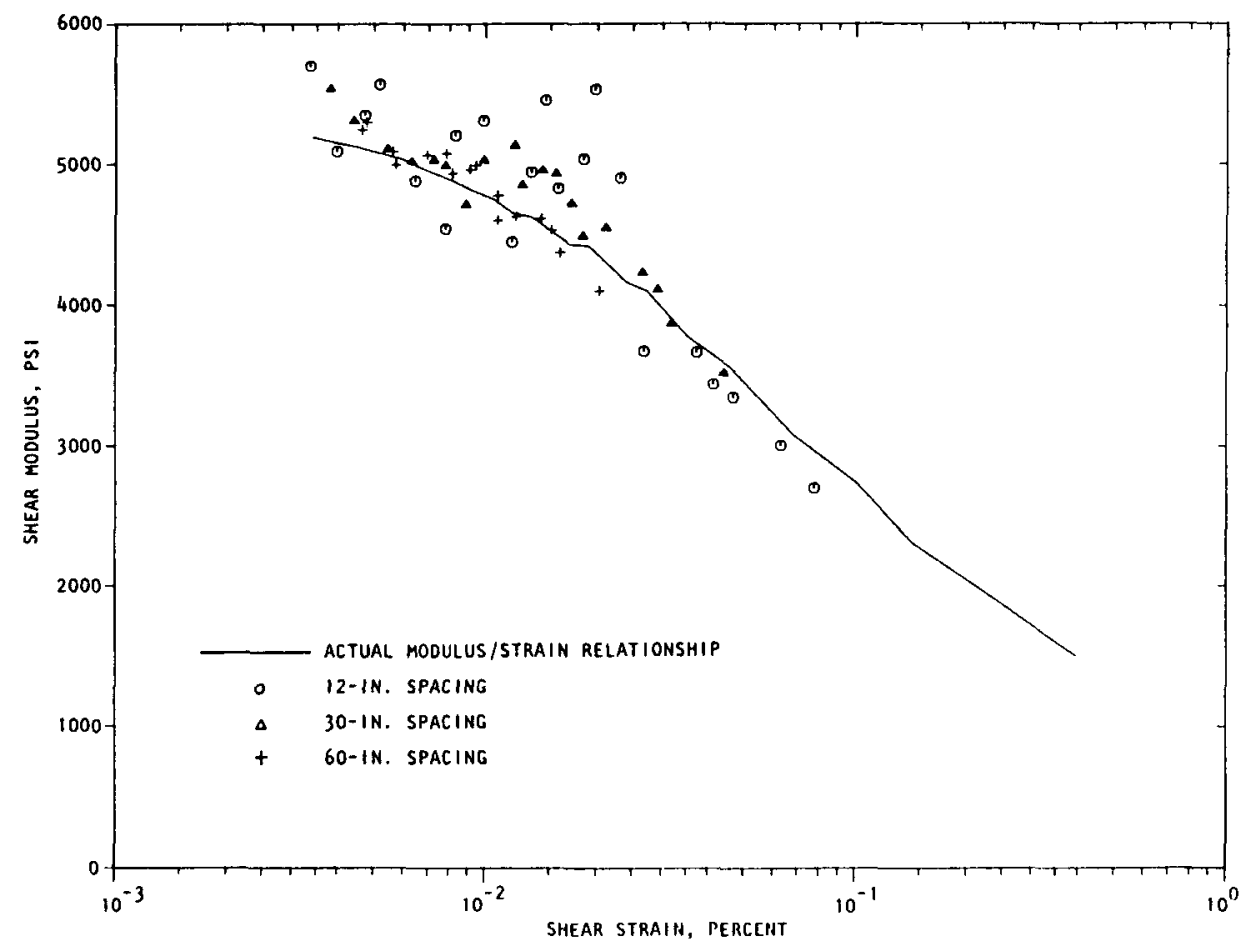

(b) Peak-velocity/midpoint method

FIGURE 4-21. USE OF MIDPOINT METHOD TO ESTIMATE SHEAR MODULUS VS. STRAIN RELATIONSHIP -- NOHLINEAR FINITE ELEMENT RESULTS 


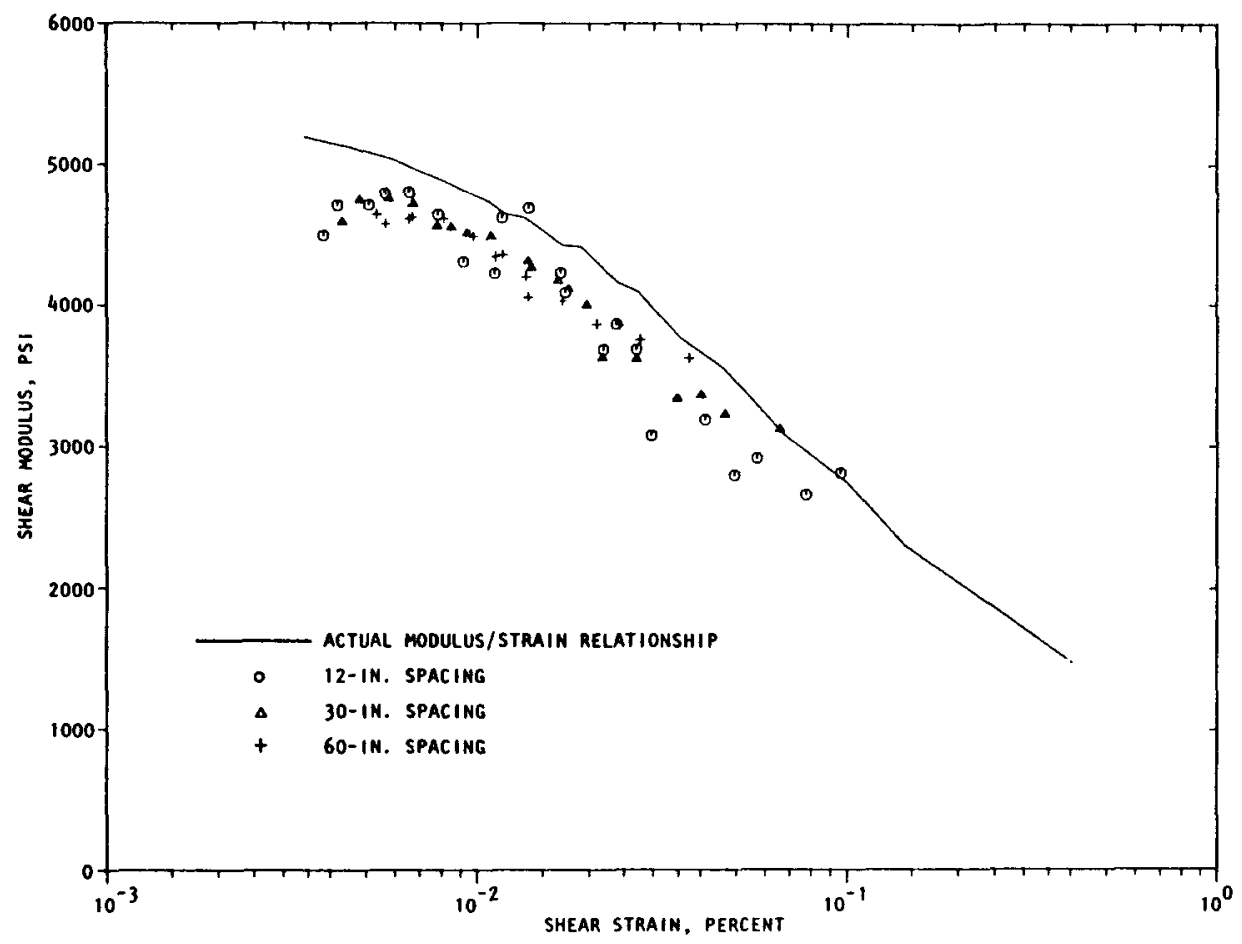

(a) Zero-crossing/integration method

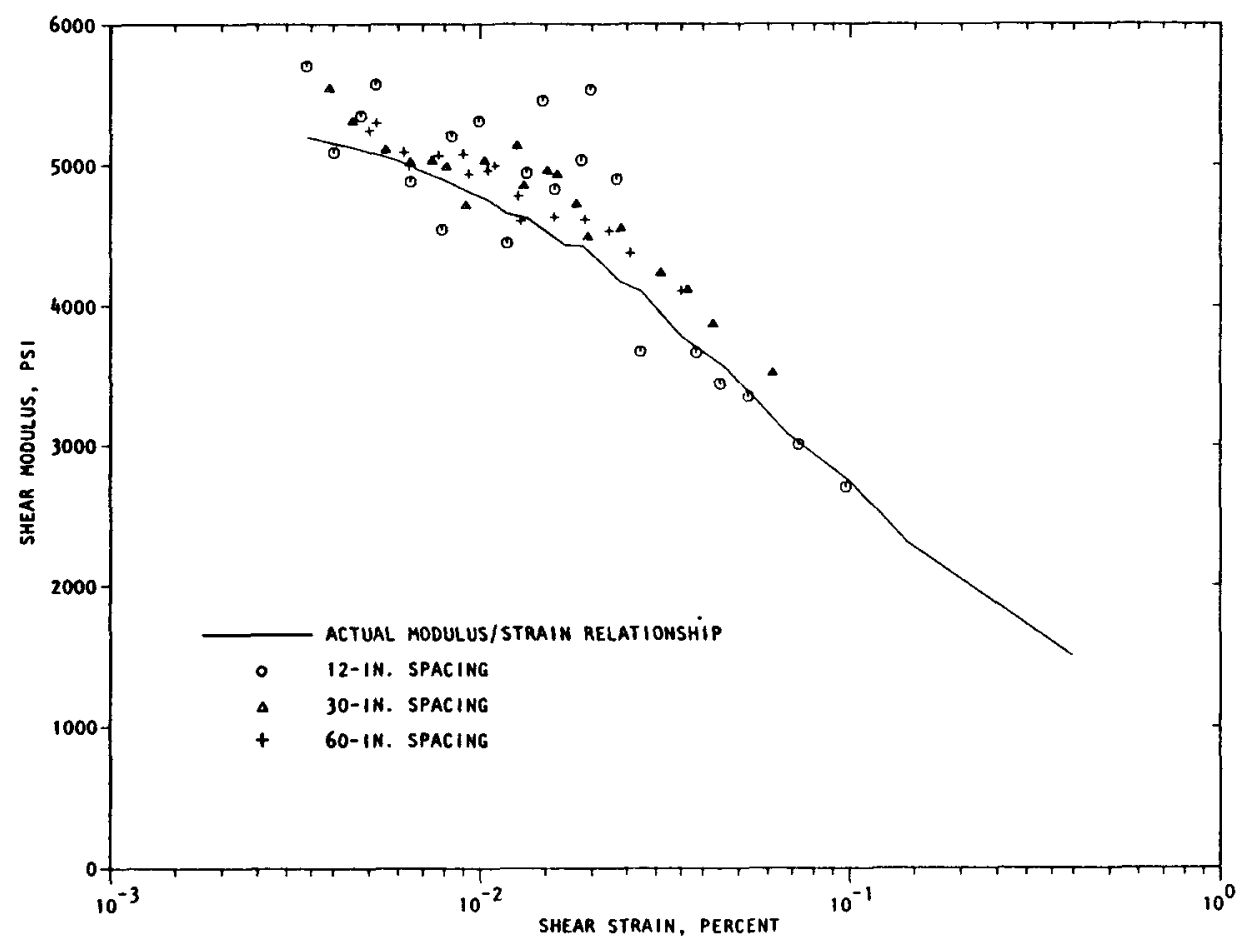

(b) Peak-velocity/integration method

FIGURE 4-22. USE OF INTEGRATION METHOD TO ESTIMATE SHEAR MODULUS VS. STRAIN RELATIONSHIP -- NONLINEAR FINITE ELEMENT RESULTS 


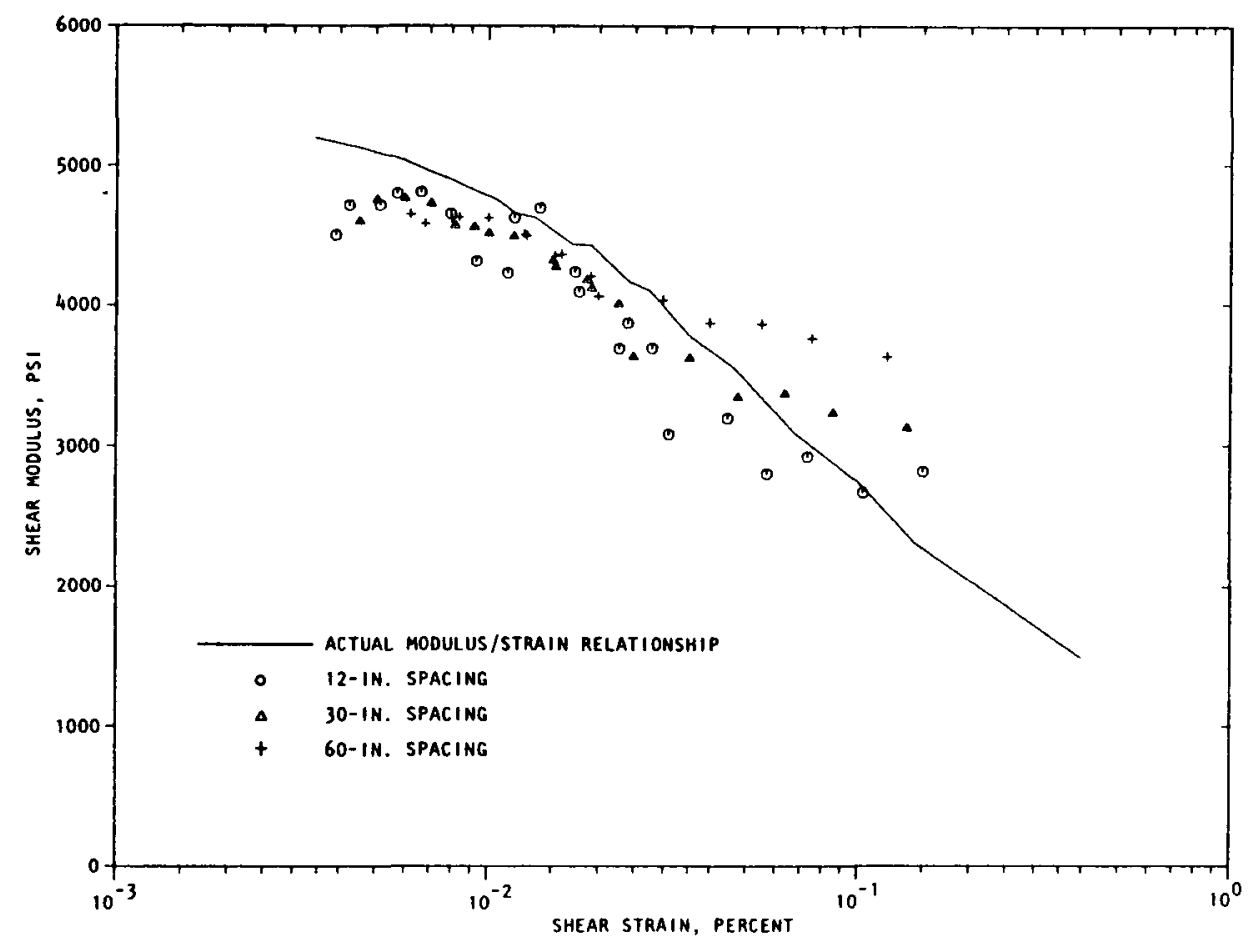

(a) Zero-crossing/end-average method

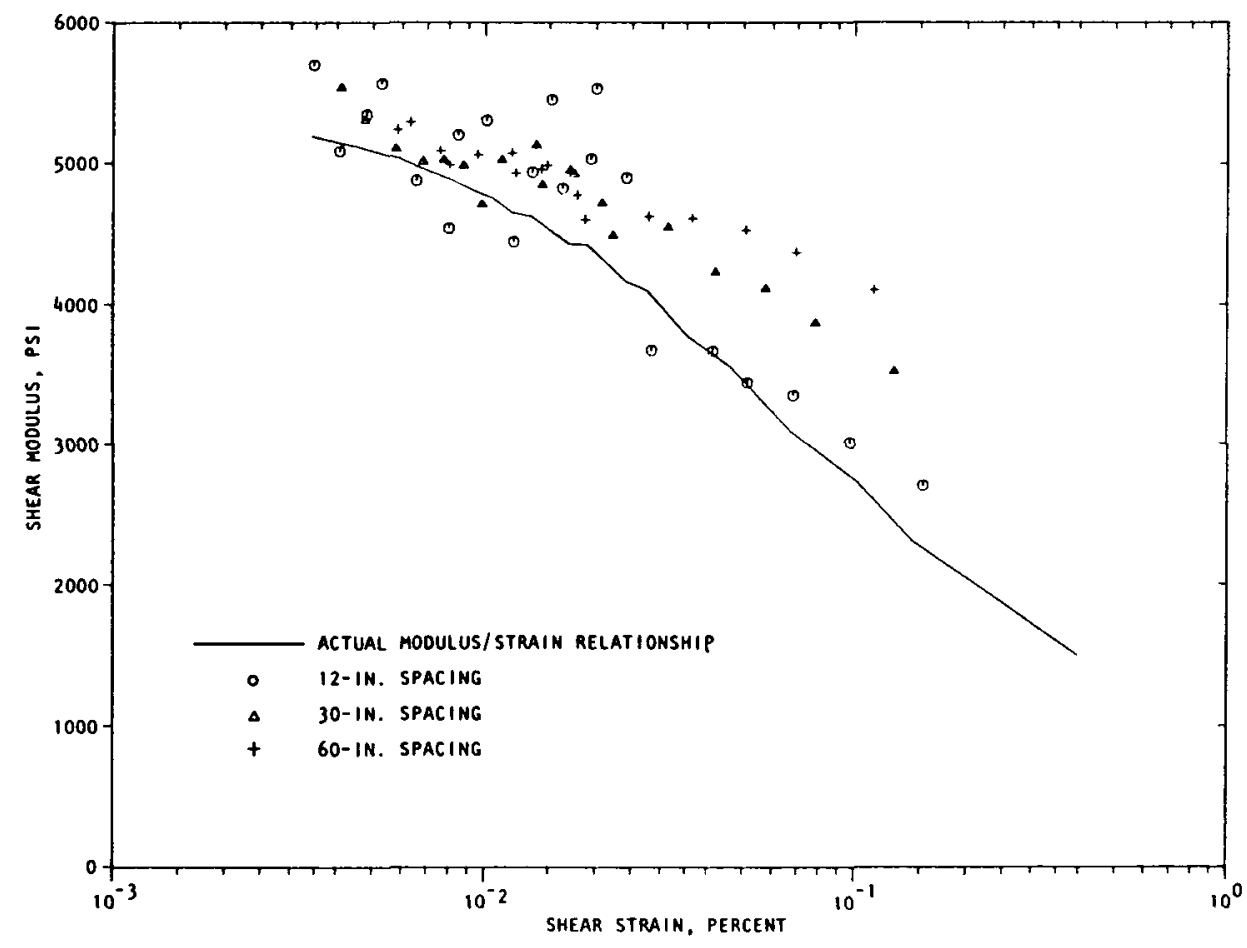

(b) Peak-velocity/end-average method

FIGURE 4-23. USE OF END AVERAGE METHOD TO ESTIMATE SHEAR MODULUS VS. STRAIH RELATIONSHIP - - NONLINEAR FINITE ELEMENT RESULTS 
in error, particularly for large strains. The other two methods appear to be reasonably good.

Fig. 4-24a shows the zero-crossing/fit method applied to a group of four nodal points spaced in a manner which might be considered for use in an actual in situ test. ${ }^{1}$ The times of zero crossing of velocity at each of the four points were used to fit the analytical approximation of Eq. 4.2. The resulting values of the undetermined coefficients are not as optimized as they would have been had all the available position-time data of zero crossing been considered in fitting the equation. However, the use of four data points is more consistent with the amount of data ordinarily available during an in situ soil test.

The availability of an analytical approximation to the propagation of the zero crossing of velocity makes possible the estimation of shear wave velocity, and hence shear modulus, at any desired distance from the anchor. The estimation of peak shear strain, on the other hand, requires also a knowledge of the peak particle velocity which occurred at the point of interest. This information is available only at the four locations for which the velocity histories are known. Only four strain values can be estimated, therefore, and as a result only four points can be plotted on the modulus/strain graph, Fig. 4-24a.

In an actual in situ test, there would be a multiple of repetitions of the test loaded under a wide range of different

1 One of these nodal points, located on the anchor, records the anchor motion. Due to the idealization of the finite element model whereby no slippage occurs between the anchor and the soil, this anchor motion is interpreted, in the data reduction procedures, as the motion of the soil along the anchor/soil interface. During an actual in situ test, where slippage may be present, this type of interpretation of the anchor motion in the data reduction procedure may not be possible. 


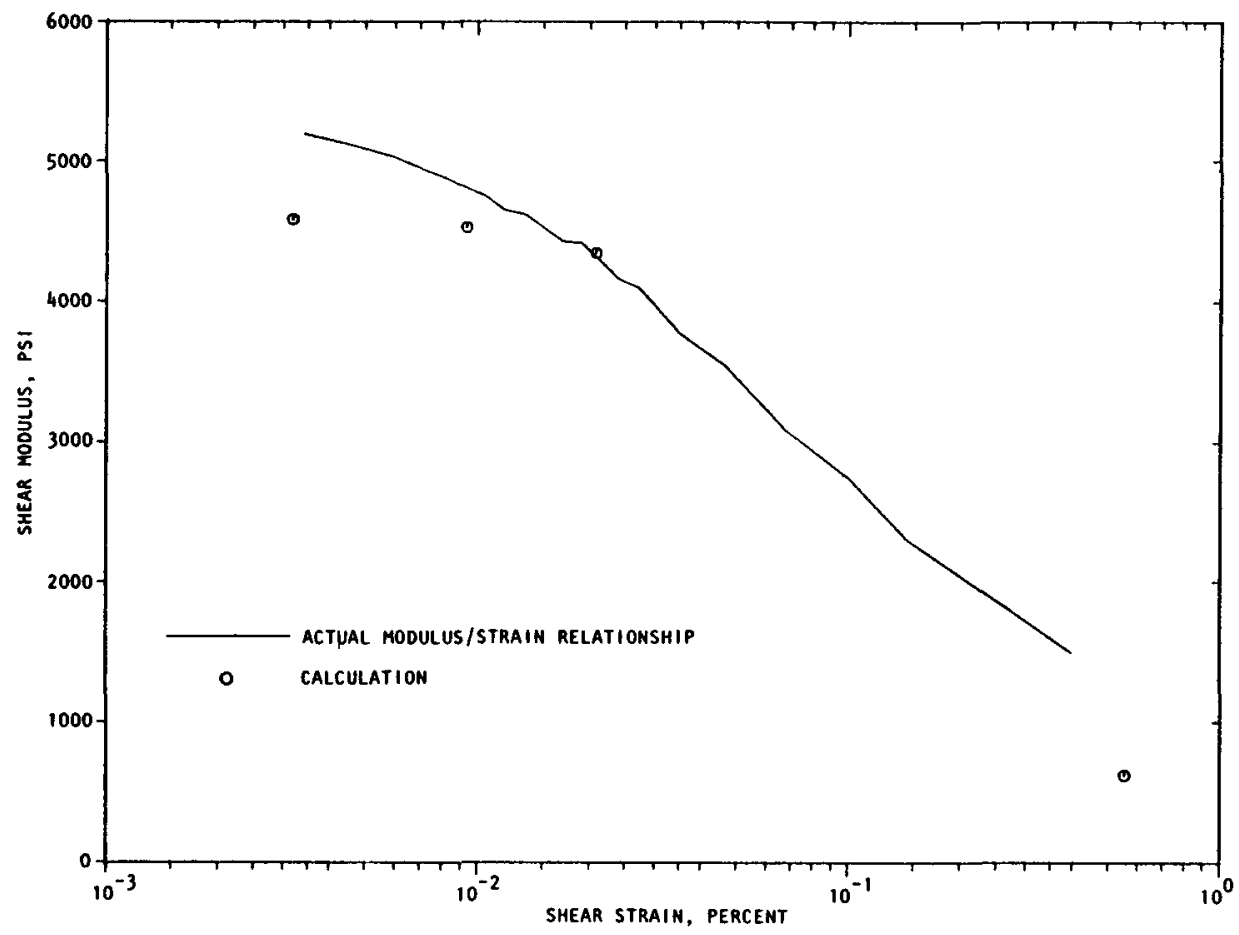

(a) Nodal points located in anchor and $30 \mathrm{in}, 60 \mathrm{in}$. , and 120 in. from the anchor

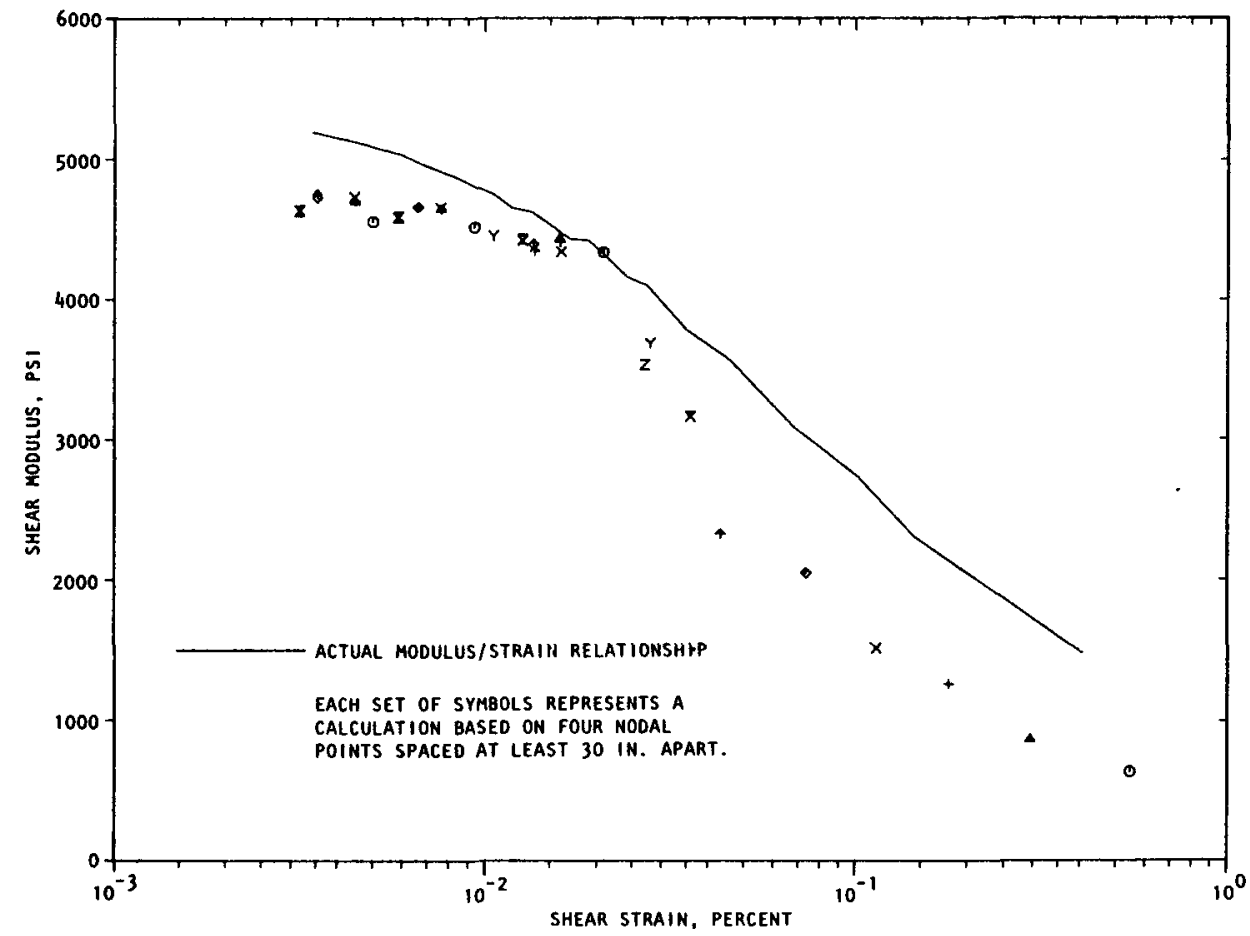

(b) Accumulation of many sets of data

FIGURE 4-24. APPLICATION OF ZERO-CROSSING/FIT IIETHOD TO NONLINEAR FINITE ELEMENT CALCULATIOH 
energy levels. This would cause a variety of strain levels to occur at the various sensor locations (which are constant from test to test), thus allowing accumulation of a large quantity of shear modulus vs. strain data distributed uniformly over a large strain range. This accumulation of data, when plotted on a single graph, would result in the modulus/strain relationship. In order to simulate such a sequence of tests it was proposed that a similar accumulation of modulus/strain data could be obtained by considering several different groups of four sensor locations spaced about 30 inches apart. The results of such a consideration of the zero-crossing data of the nonlinear finite element calculation are shown in Fig. 4-24b.

An interesting feature of Fig. 4-24 is the small discrepancy between the data reduction technique and the actual modulus/strain relationship for small values of peak shear strain. The small values of shear strain occur at large distances from the anchor, where the method of computing shear strain has been shown to give excellent results (see Fig. 4-19). This suggests that the method for computing shear wave velocity (from which the shear modulus is computed) may be in error.

Due to the nonlinearity of the soil medium, the velocity of propagation of shear waves at a given location in the soil is constantly changing according to the changing state of stress at that location. At any given instant of time, the relation:

$$
\mathrm{V}_{\mathbf{S}}=\sqrt{\mathrm{G} / \rho}
$$

which is obtained by rearranging Eq. 4.1, holds true. The solid curves of Fig. 4-24, which are labeled "actual", show the relationship between secant shear modulus and peak shear strain. In order to obtain wave propagation data which agree with these curves, the feature being observed would have to be propagating 
at the secant shear wave velocity, which is obtained by using the secant shear modulus in place of $G$ in Eq. 4.5. It is evident from Fig. 4-24 that the zero crossing of velocity propagates at a velocity slower than the secant shear wave velocity at large distances from the anchor. This observation is confirmed in Figs. 4-14a and 4-16a.

Fig. 4-25a shows the peak-velocity/fit method applied to the same sensors used in Fig. 4-24a. Fig. 4-25b shows an accumulation of peak-velocity/fit data. By comparison with Figs. 4-2lb, 4-22b, and 4-23b the scatter is greatly reduced using the fit methods. Furthermore, the strain range is much more extensive. Otherwise the modulus/strain relationships obtained from the two sets of data are very much in agreement. Fig. 4-25b shows that there is a range of locations for which the velocity of propagation of the peak velocity exceeds the secant shear wave velocity, but that the two approach each other toward the outermost locations considered in the calculation. This observation is consistent with Figs. 4-14b and 4-16b.

\subsection{CONCLUSIONS}

Based on the results presented in this chapter, a number of conclusions are listed below. When interpreting these conclusions, the inherent advantages and limitations of the finite element procedures, as aiscussed in section 4.1.1, should be kept in mind. Furthermore, to aid in this interpretation, certain comments based on experimental results from the special tests are provided as footnotes. These experimental results are described in more detail in chapter 5 .

With this as background, the conclusions regarding the finite element analysis results are as follows: 


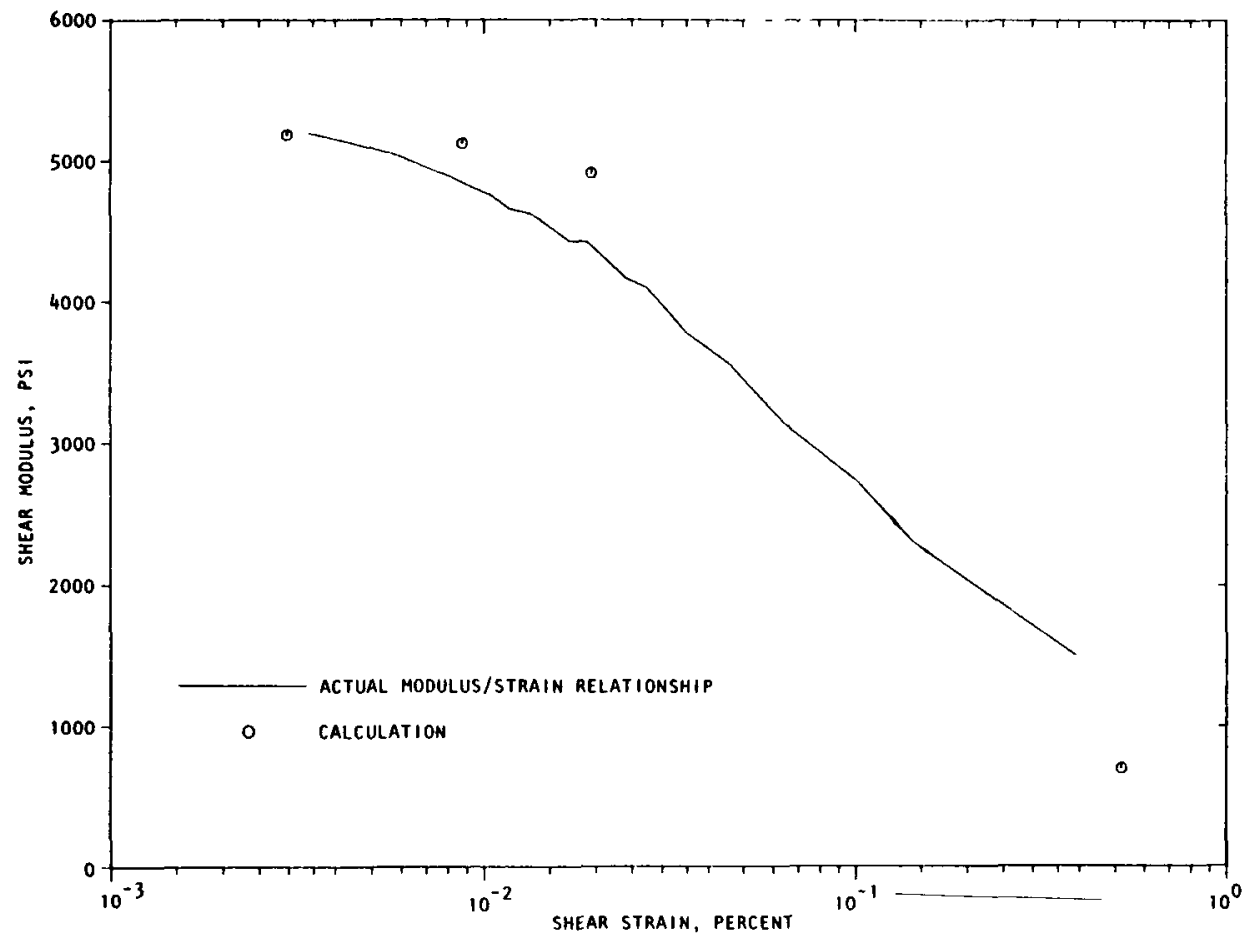

(a) Nodal points located in anchor and $30 \mathrm{in.,} 60 \mathrm{in}$, , and $120 \mathrm{in}$. from the anchor

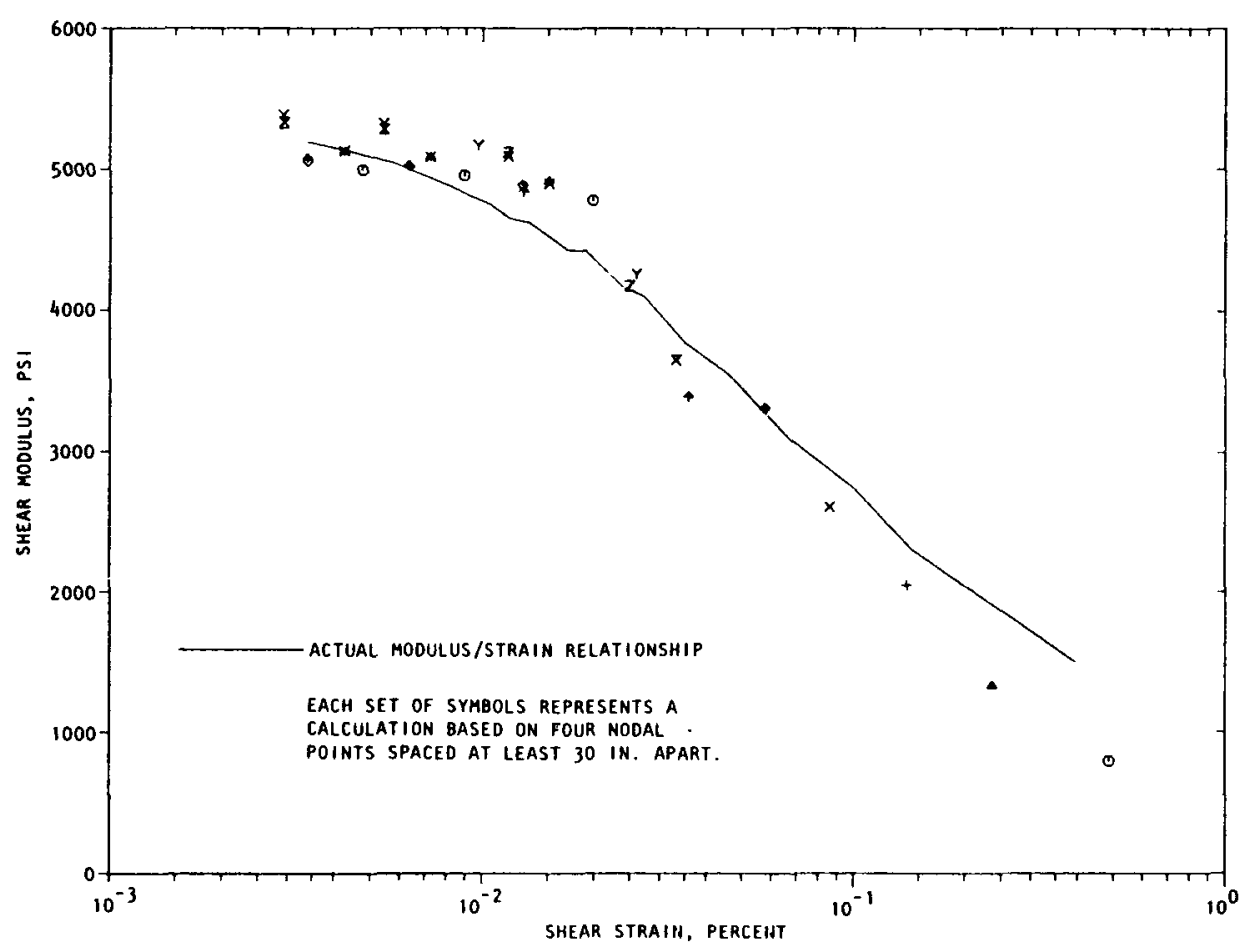

(b) Accumulation of many sets of data

FIGURE 4-25. APPLICATION OF PEAK-VELOCITY/FIT METHOD TO NONLINEAR FINITE ELEMENT CALCULATION 
a. Although time-history methods involving zero-crossing and peak-velocity produce slightly different results, both methods are satisfactorily accurate. The results of the peakvelocity methods appeared to be slightly more accurate in the particular nonlinear calculations described in this chapter; however this may not necessarily be true for other soil conditions or input loads. In fact the zero-crossing method was superior in analyzing the data from the elastic case.*

b. The time-history methods include both curve-fit methods and travel-time methods, as shown in Table 4-l. The curve-fit methods are able to extract a wider range of data from the experiments by making use of the near-anchor soil region. The various data processing techniques have been shown to be somewhat less reliable near the anchor than far from it. Despite these potential near-anchor problems, the modulus/strain comparisons for the curve-fit methods were found to be satisfactory near the anchor in the case of the nonlinear finite element calculation.

c. The travel-time methods are subject to excessive scatter, due to numerical differentiation incorporated within the methods. The nonlinear finite element calculations show that the sensor spacing must be about 30 inches or more in order to reauce the scatter to acceptable levels. This minimum allowable sensor spacing will probably vary from test to test depending upon such factors as shear wave velocity, input energy level, etc. It is evident from the modulus/strain comparisons that increased sensor spacing reduces the range of data obtainable. Thus, these methods are judged to be less useful than the curve-fit methods.

It was observed in Section 5.3 that the zero-crossing data can be somewhat more precisely resolved from the special test measurements than peak velocity data. The two methods, when applied to production tests, gave nearly the same results. 
d. The wave-form methods were shown to be inappropriate for the calculation of the shear wave velocity in the near-anchor region.* Away from the anchor, these methods were shown to produce satisfactory results which agree with those of the timehistory methods. However, because of the importance of the near-anchor region and the uncertainties associated with the application of wave-form methods for estimating shear wave velocities in this region, these methods are not recommended for use in production tests.

e. The use of Eq. 4.3 to estimate peak shear strain is extremely successful away from the anchor.** In the near-anchor region, the performance of these formulae is only moderately successful. Errors in the near-anchor region evidently do not exceed 50\%, and are often much smaller. The end-average method of determining the average strain for a region between sensors is not recommended.

f. On the basis of the modulus vs. strain comparisons, the curve-fit methods are recommended for processing of in situ data. Referring to 'íable 4-1, these methods include the zerocrossing/fit method and the peak-velocity/fit method.

As noted in section 5.3.2, wave-form methods have an additional disadvantage when used to estimate near-anchor shear wave velocities during typical production tests -- namely, the spatial resolution of the near-anchor soil sensors is not sufficient to provide an adequate representation of the crest of the wave in this region. However, the special tests described in chapter 5 were different from typical production tests in that they provided an extremely fine spacing of nearanchor sensors that permitted an adequate representation of the displacement wave shape in this region. Therefore, the slopes of these displacement profiles were used in Chapter 5 to determine shear strains generated in the soil during the special tests. Such a treatment follows directly from the definition of shear strain and is valid even though the waveform methods may be inappropriate for use in estimating nearanchor shear wave veloclties.

**

As discussed in section 5.4 of Chapter 5, analysis of the special test data confirms the validity of the current methods for estimating shear strain. 
CHAPTER 5

EXPERIMENTAL STUDY OF WAVE

PROPAGATION IN AN ARTIFICIAL SOIL FILL

Special field tests were carried out to provide additional insight into the wave propagation phenomenology of the "real" in situ test condition particularly for the region close to the anchor. This work was accomplished by installing the anchor assembly and extensive monitoring equipment within a soil fill built for this purpose and conducting tests under a variety of controlled test conditions. The results of these studies are described in this section. While many tests were performed and much data was reduced and studied as part of this effort, for clarity, data for only representative cases are included in this chapter. More detailed treatment of this data has been presented in Troncoso, 1975.

\subsection{TEST DESCRIPTION}

The testing program was conducted in soils at a site on the Fort Lawton Military Reservation in Seattle, Washington. The soils used in the test fill consisted of a relatively uniform deposit of silty fine to medium SAIND. The index, mechanical and dynamic properties of this material, based on a number of laboratory tests are provided in Appendix $\mathrm{C}$.

At this site, a large circular pit was first excavated to the dimensions shown in Figure 5-1. These dimensions were selected to avoid interference of reflected waves from the boundaries. The pit was then backfilled, and the materials densely compacted in thin layers for the entire depth of the pit. During the backfilling operation, the anchor assembly (Fig. 5-1) and a number of recording sensors consisting of four accelerometers, 34 velocity transducers and one load cell, were hand placed at predetermined locations in the soil fill. Six of these sensors 


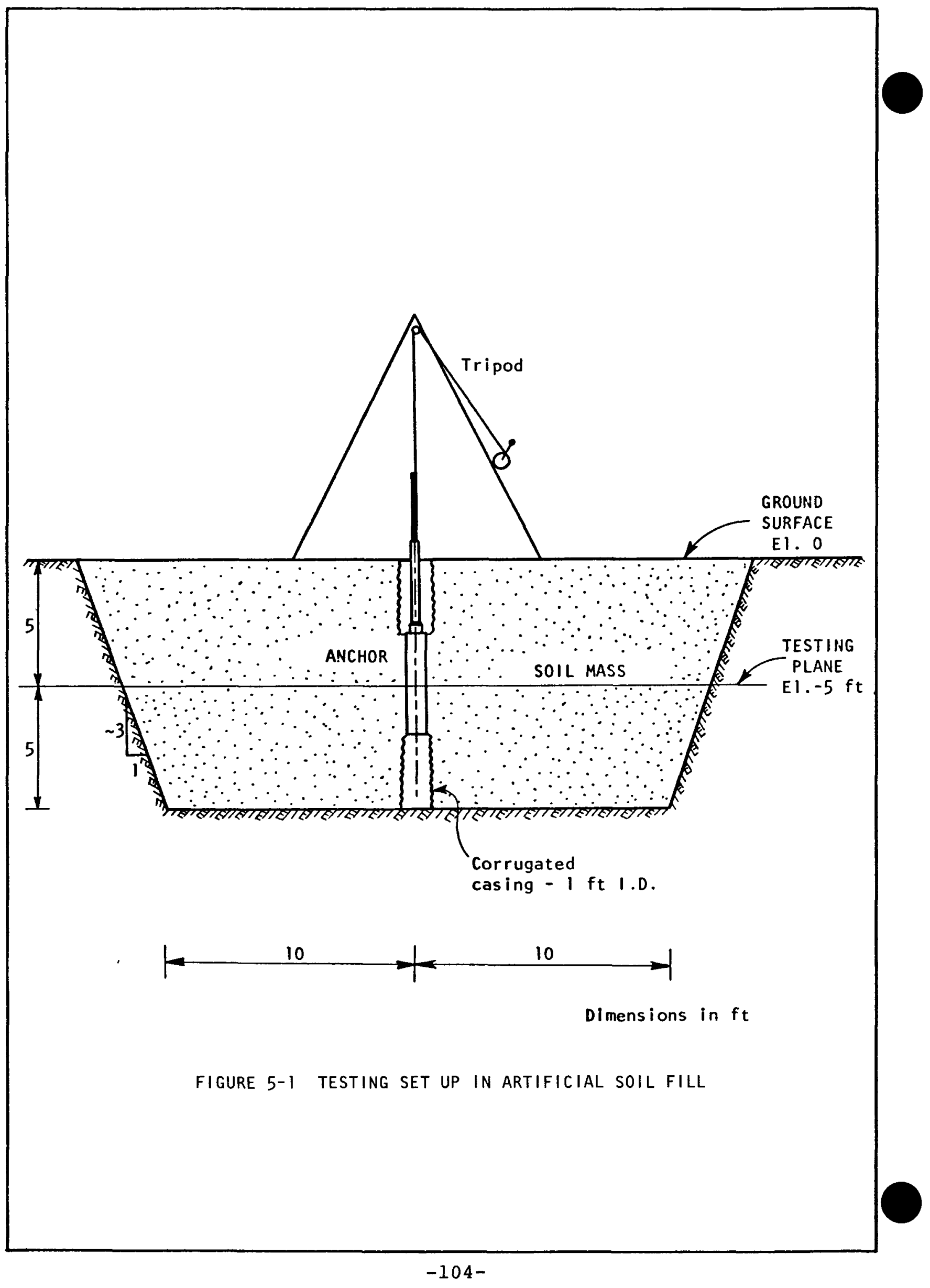


were placed on the anchor assembly. Most of the remaining sensors were located at the mid-depth of the pit (elevation -5 feet). The type and location of these sensors are identified in Fig. 5-2. Further details of fill construction and equipment installation procedures including photographs are providea in Appendix D.

The band of sensors in Fig. 5-2 was placed primarily to study wave propagation characteristics, and to provide insight into data interpretation procedures. As indicated in Appendix E, these sensors were staggered to avoid possible interaction effects between adjacent sensors. Also from this same band of sensors, test parameters such as P-wave effects (from the horizontally oriented sensors), and repeatability, could be studied. The remaining array of free field sensors together with the band provided data for study of symmetry and casing effects. Simultaneous measurements of motion and forces at the anchor and the close-in free field stations provided data for better understanding anchor behavior characteristics particularly for establishing empirical relationships relating measurements made at the anchor to those recorded in the free field. Also the importance of radial jacking stresses, and hammer weight and height of drop effects could be assessed. Most of these test parameter effects are discussed and evaluated in Appendices $F$ and $G$.

Because the recording equipment is capable of monitoring only four sensors at a time, any test involving more than four measurements had to be repeated in order to collect sufficient data. By re-recording in every two tests at least one data station, normalization of times and amplitudes could be readily accomplished. 'i'he observed variations in amplitude and shape were almost always small and time shift adjustments between successive records were easily accomplished. With repeated tests, under constant test conditions, the response characteristics as 


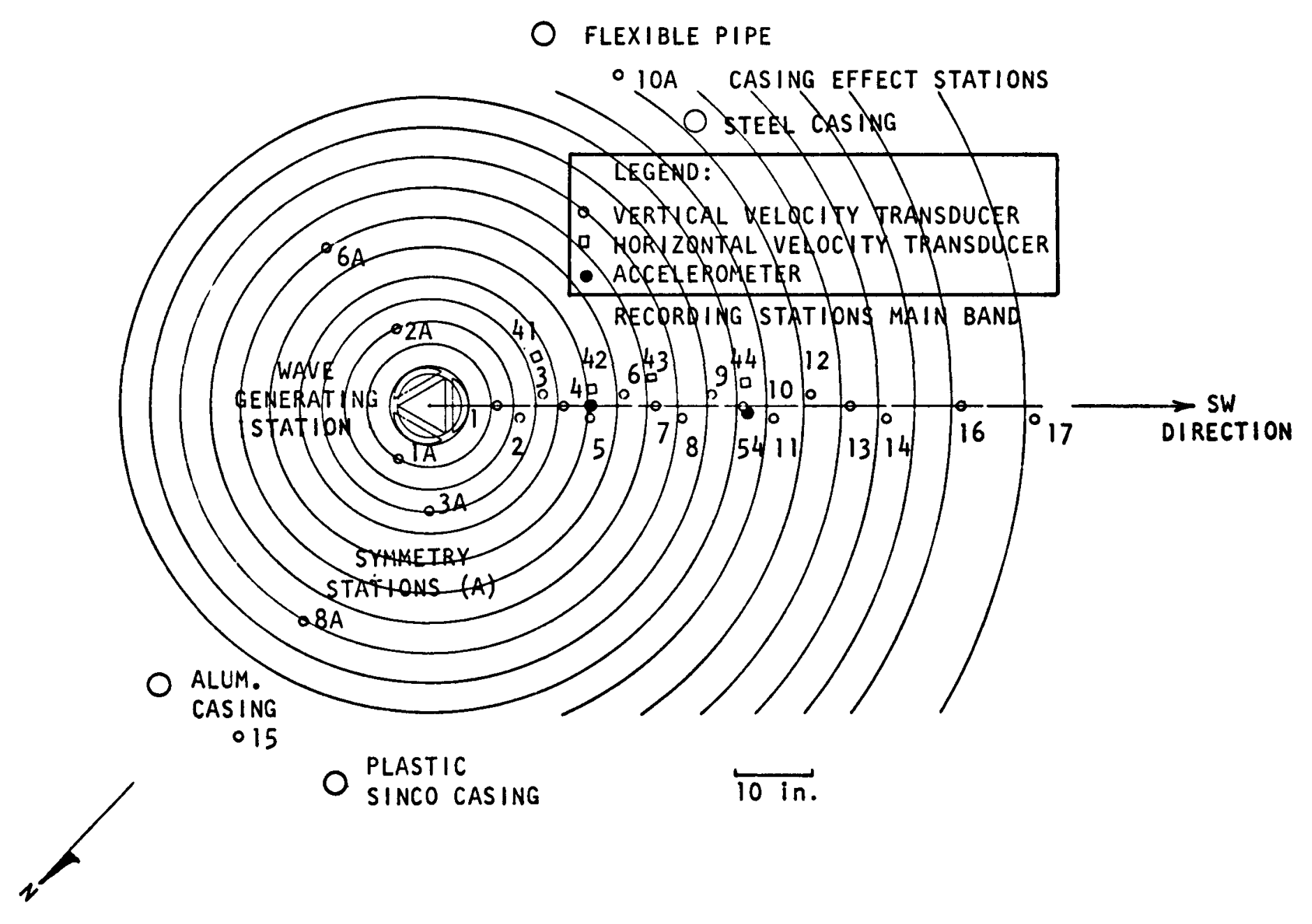

FIGURE 5-2 SENSOR LOCATION PLAN AT ELEVATION - $5 \mathrm{ft}$ 
the wave travels from the source through the band of sensors could be studied as if the data had all been obtained from one impact.

Test results for all sensors were obtained as time-histories of loads, accelerations and both horizontal and vertical particle velocities at different points in the soil and in the anchor. Through integration and other mathematical manipulations of the test data together with accurate distance measurements, displacements were determined and related as wave-forms both in terms of time and distance. This data provided a clear picture of changing wave characteristics and enabled actual wave velocities and shear strains to be computed. The data also enabled the laws of attenuation to be studied to a limited extent and provided for relating free field and anchor motion data.

\subsection{WAVE PROPAGATION CHARACTERISTICS}

5.2.1 Particle Velocity Characteristics

Wave propagation was mainly studied by measurements of the responses of 16 vertical velocity sensors arranged along the principal band shown in Fig. 5-2. A complete series of tests performed at a constant arop height, hammer weight, and jack pressure, $\sigma_{j}$, are shown in Fig. $5-3$ as 16 velocity time measurements as the wave passes outward from the energy source. ine most characteristic points of the velocity measurement are defined as shown in the idealized time history in Fig. 5-4. This idealized pulse is a superposition of vertical particle velocities generated by the passage of both compressional or $P$ waves and shear or $S$ waves. The first part of the pulse will be defined primary pulse and the second main pulse.

The curves in Fig. 5-3 illustrate progressive changes in the shape of the pulses as they travel outward through the 


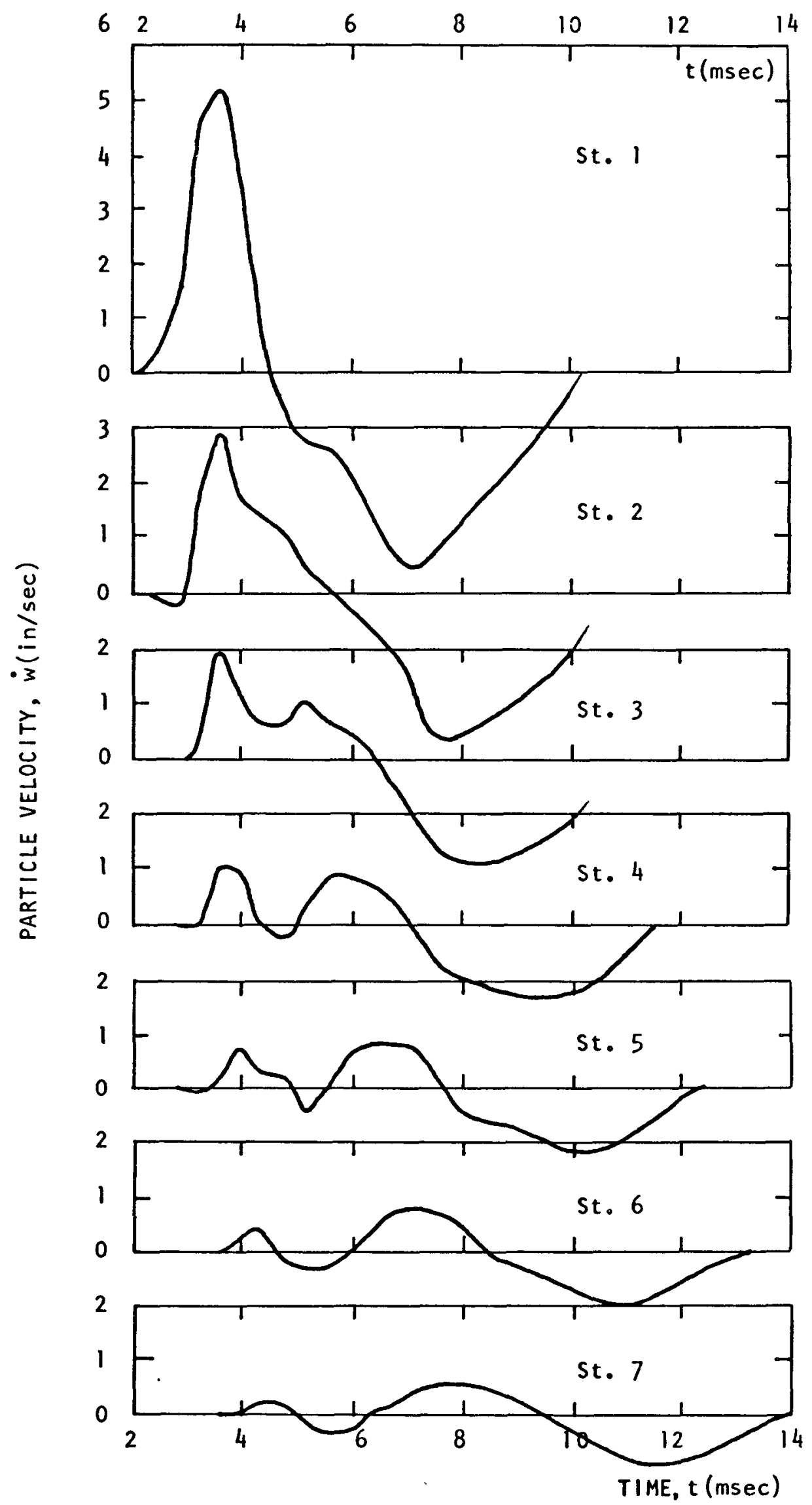

FIGURE 5-3à PARTICLE VELOCITY - TIME HISTORIES

TESTS $2.2 \mathrm{~A}-2.8 \quad W=58 \mathrm{lb} \times .5 \mathrm{ft}, \sigma_{j}=2,000 \mathrm{psi}$ 


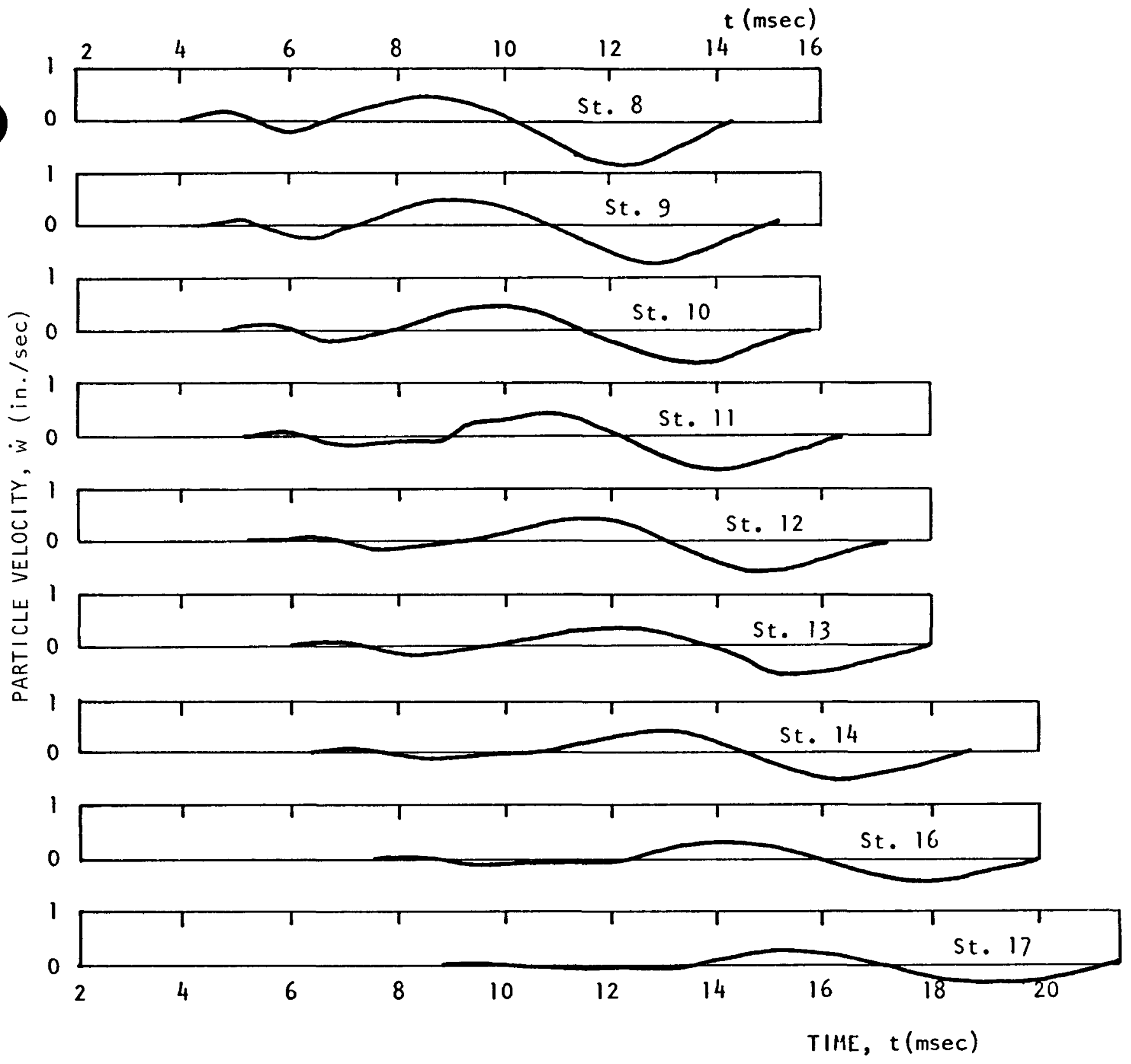

FIGURE 5-3b PARTICLE VELOCITY - TIME HISTORIES

TESTS $2.2 \mathrm{~A}-2.8 \mathrm{~W}=58 \mathrm{lb} \times .5 \mathrm{ft}, \sigma_{j}=2,000 \mathrm{psi}$ 


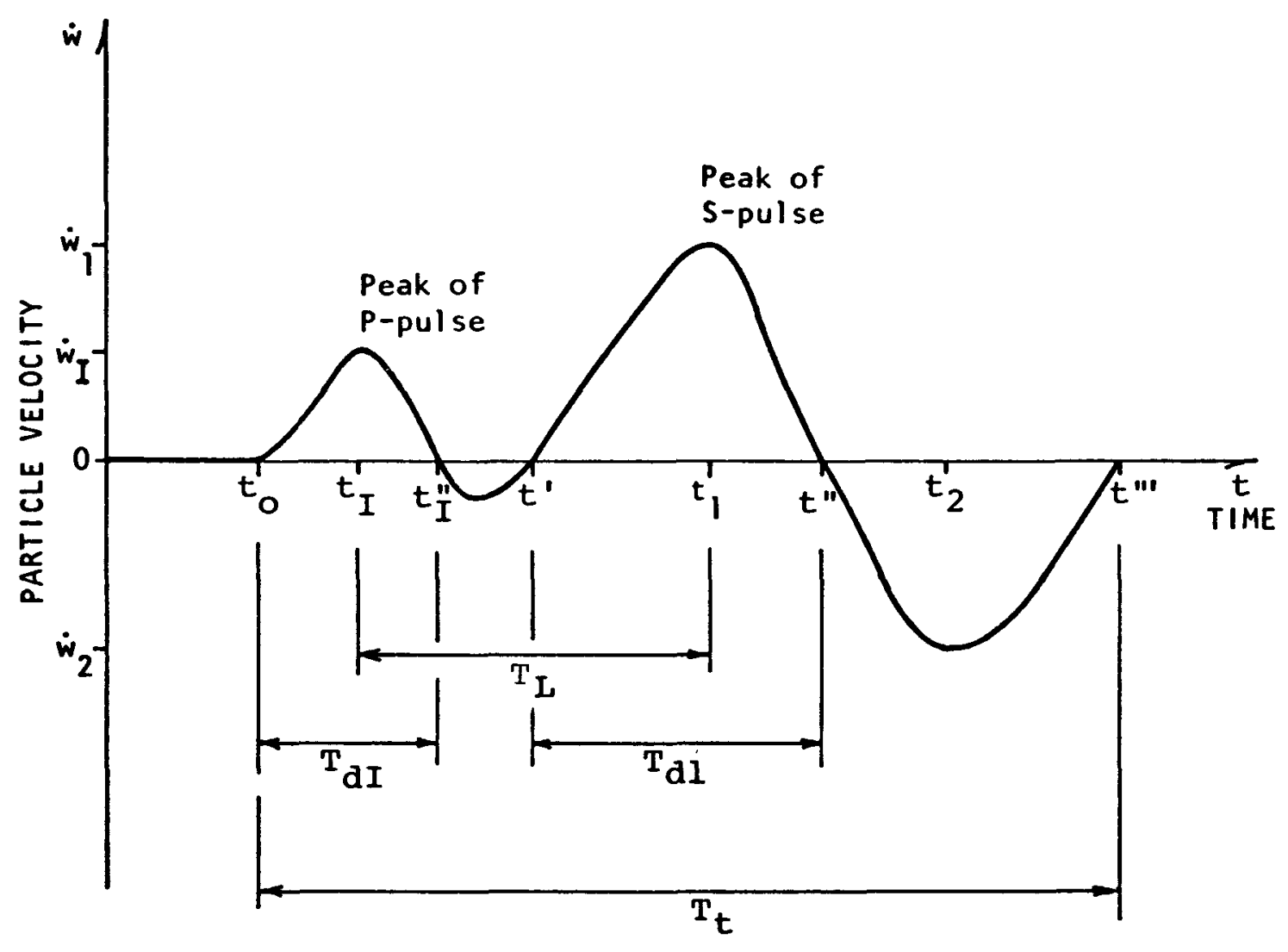

Sign Convention:

Positive for downward motion

Time definitions:

$t_{0}$ : first arrival

$t_{1}^{\prime \prime}$ : second crossing of $P$-pulse

$t$ ': arrival of S-pulse

$t^{\prime \prime \prime}$ : end of S-pulse

$T_{d l}=t_{l}^{\prime \prime}-t_{0}$ : duration time of positive part of $P$-pulse

$T_{d l}=t^{\prime \prime}-t^{\prime}$ : duration time of positive part of $S$-pulse

$T_{L}=t_{1}-t_{1}$ : time lag between $P$ and $S$ pulses

$T_{t}=t^{\prime \prime \prime}-t_{0}$ : total duration time of complete pulse

FIGURE 5-4 TYPICAL PARTICLE VELOCITY - TIME HISTORY 
soil mass. In general the particle velocity signatures change from one composite pulse to the two distinct primary and main components defined in the idealized curve (Fig. 5-4). As this change or separation is taking place, there is also an increase in the time durations of both the main pulse and the total pulse. This indicates that the two components are traveling at different speeds. The primary component is traveling the fastest and is therefore the $\mathrm{P}$-wave while the slower traveling main component represents shear wave motion. Appropriate substitution of Poisson's ratio in the theoretical equation:

$$
\frac{V_{p}}{V_{s}}=\sqrt{\frac{2(1-\mu)}{1-2 \mu}}
$$

will always produce a ratio greater than 1 (i.e., the P-wave is always the faster of the two body waves).

Further examination of the set of waves reveals that the primary pulse is dominant in the "close in" zone around the anchor, but becomes much smaller at greater distance. This indicates that for these distances, the primary component (P-wave) attenuates much faster (about 10 times) than the main component (S-wave).

To estimate the point in this set of curves where the two components separate, the following example is provided.

For a soil with an average s-wave velocity of 400 $\mathrm{ft} / \mathrm{sec}$ and $\mu=.3$, the $\mathrm{P}$-wave velocity $V_{p}$ is computed to be $750 \mathrm{ft} / \mathrm{sec}$. Using the terms in Fig. 5-4, approximate conditions for no overlap of the pulses may be expressed as:

$$
\Delta t=t_{I}-t_{I} \geq \frac{{ }^{\mathrm{I}} \mathrm{dl}+\mathrm{T}_{\mathrm{dl}}}{2}
$$


For: $\quad t_{1}=\frac{r}{v_{s}} \quad$ and: $\quad t_{I}=\frac{r}{v_{p}}$

where $r$ is the radial distance of the points of no overlap,

$$
r \geq \frac{1}{2} \frac{\left(\mathrm{T}_{\mathrm{dl}}+\mathrm{T}_{\mathrm{dl}}\right)}{\left(\frac{1}{\mathrm{~V}_{\mathrm{s}}}-\frac{1}{\mathrm{~V}_{\mathrm{p}}}\right)}
$$

Using this relationship and assuming both $\mathrm{T}_{\mathrm{ds}}$ and $\mathrm{T}_{\mathrm{d}}$ are 2 msec, the radial distance of no overlap is 1.8 feet. This corresponds to a point located between stations 5 and 6 , in Fig. 5-2. Based on this example calculation and study of other test series, the zone of no overlap generally occurs at a distance from the edge of the anchor varying between about 1.5 to 2.0 feet. Thus at distances greater than this, the main pulse is clearly dominant and unaffected by large P-wave effects. This outer zone may be defined as the "Eree field".

5.2.2 Displacement Characteristics

The displacement characteristics for studying wave propagation can be presented in two ways, either in relation to time or distance. Direct integration of the velocity-time histories produces pulses of displacement or displacement-time histories. Fig. 5-5 shows a typical set of displacement pulses obtained for a 58 pound weight falling 0.5 feet, and a constant jack pressure, $\sigma_{j}=3,500 \mathrm{psi}$. The sign convention is negative for a downward displacement. The figure shows only the negative parts of the pulses for both the anchor and the 16 vertical velocity transducers along the main band. In the "close in" zone (x.e., inside station 5) the primary pulse has a strong influence on both the positive and negative side of the displacement curve. Because of this overlapping effect, interpre- 


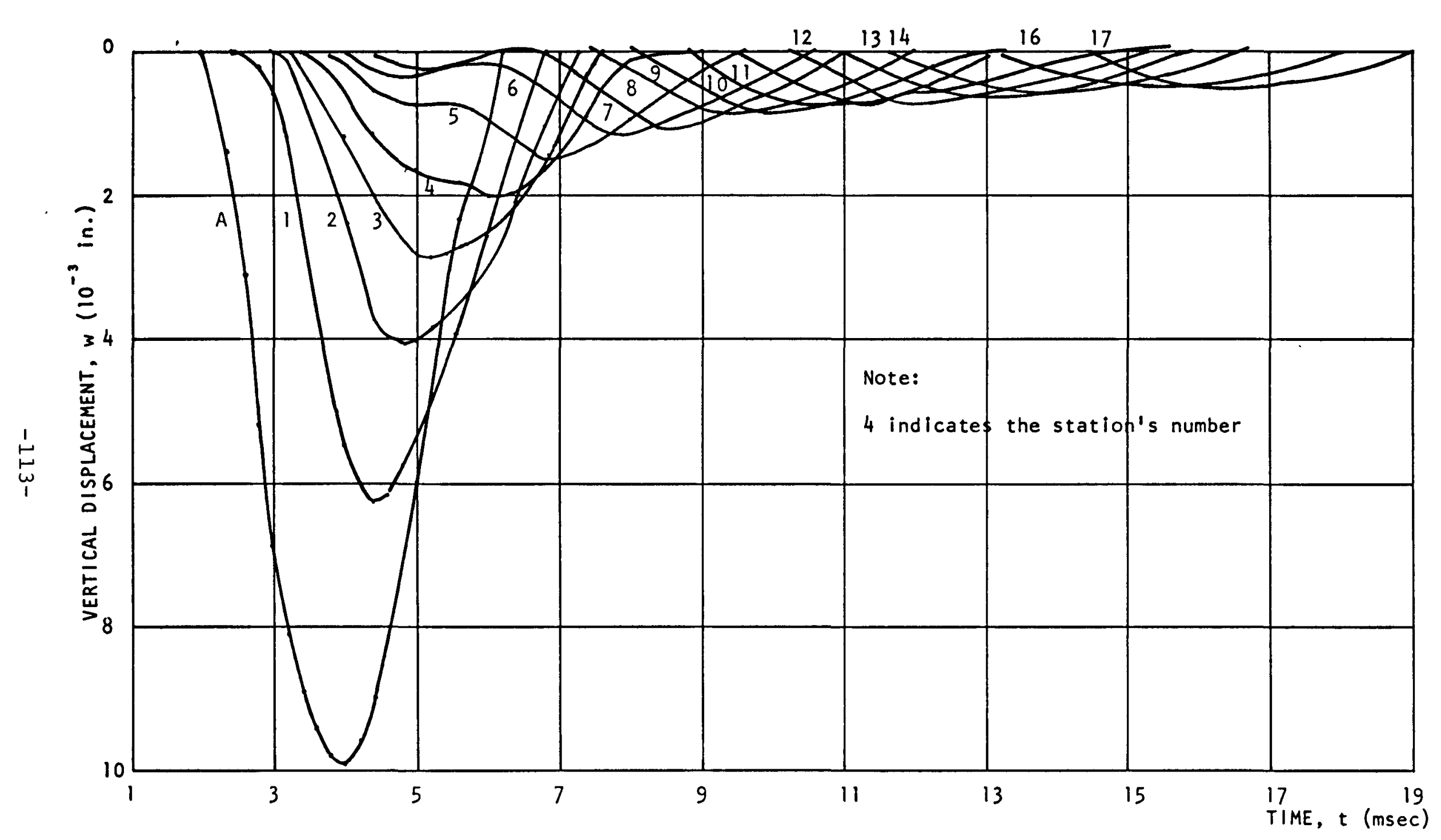

FIGURE 5-5 ATTENUATION OF DISPLACEMENTS - TESTS 6.1 to $6.7, \mathrm{~W}=58 \mathrm{lb} \times .5 \mathrm{ft}, \sigma_{j}=3,500 \mathrm{psi}$ 
tation of the shear characteristics of the wave are difficult to isolate in this region. This pulse because it is composite and is rapidly separating in the "close in" region, produces distortions also in the displacement pulse as noted in Fig. 5-5.

The data obtained from Fig. 5-5 can be revised, and presented as waveforms or isochrone curves of displacement distance plots. Fig. 5-6 illustrates the waveforms obtained from this same test data. The zone of strong attenuation corresponds to the "close in" zone (i.e., Stations 1 to 5). The rate of attenuation decreases between stations 5 and 12 and becomes very small beyond 12 . This rapid change in displacement and attenuation together with the gradual increase in the length of the wave, in effect, produces a skewed wave shape.

Except in near-anchor regions where the waveform is distorted, the waveforms provide two factors essential for "free field" data interpretations. First it shows the physical vertical shearing distortions taking place at any interval of time. They also provide a means for estimating the maximum shear strains, since the waveform itself is a curve which satisfies the equation of displacement:

$$
w=f(r, t)
$$

If the small horizontal displacements are disregarded, the slopes of these curves therefore represent the shear strains at a certain point and time or:

$$
Y=\frac{s W}{\sigma r} t
$$

Thus a maximum strain value is determined for each time isochrone at a point (or distance) corresponding to the maximum slope of that curve. This relationship is especially valid for 
STATIONS

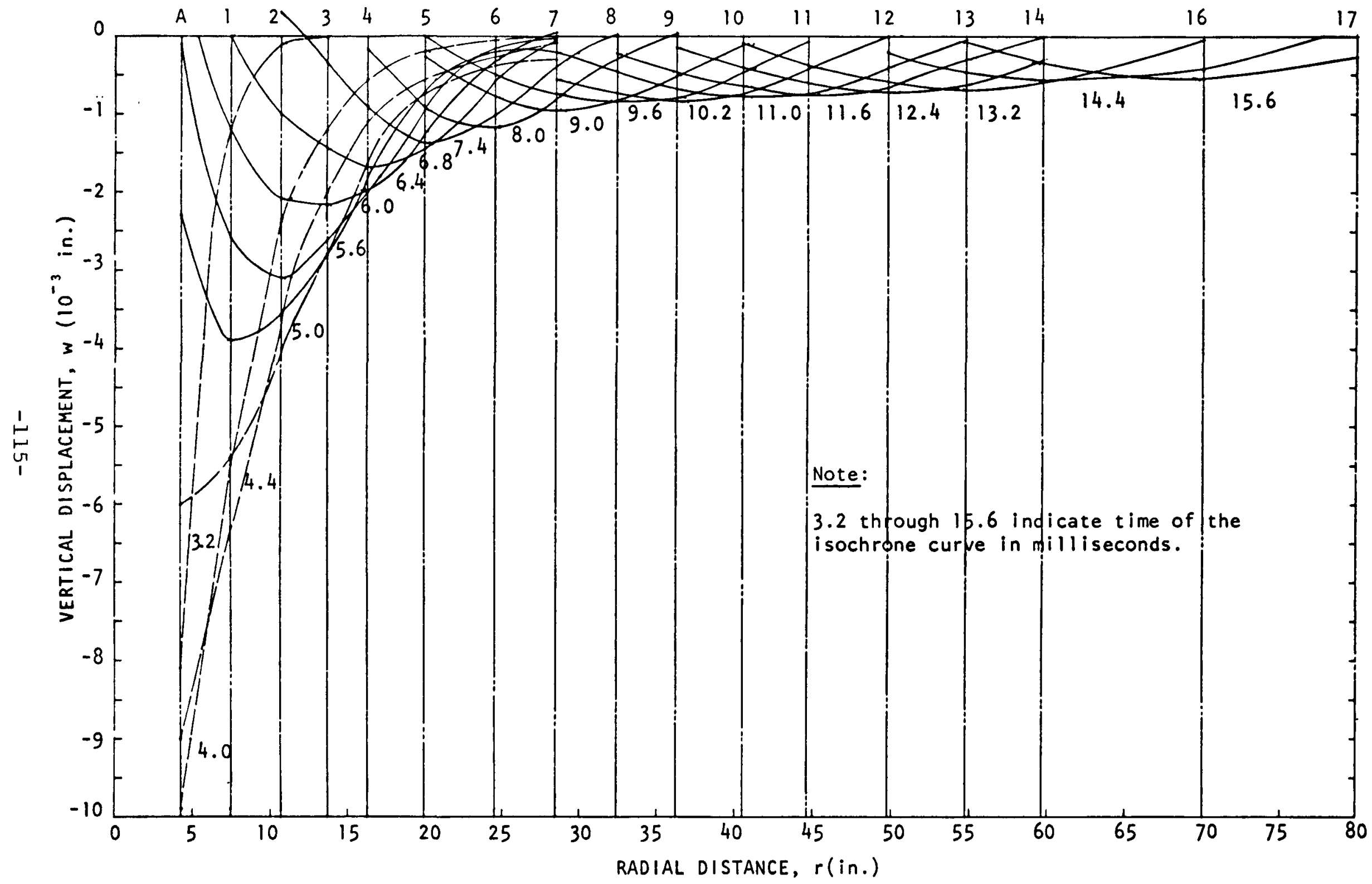

FIGURE 5-6 WAVEFORMS. TESTS 6.1 to $6.7, \mathrm{~W}=58 \mathrm{lb} \times .5 \mathrm{ft}, \sigma_{j}=3,500 \mathrm{psi}$ 
the free field sensors where the primary pulse is unimportant. It also provides a check of the validity of using plane wave theory to compute strains at the anchor. Further discussion of this strain relationship is presented in section 5.4 .

\subsection{SHEAR NAVE VELOCITY IN FREE FIELD SOIL}

The waveforms in general indicate that the most readily recognizable single point on each curve for determining propagation velocities in the free field corresponds to the crest of the wave, $r_{C}$. This crest, in general, is defined as the point of maximum displacement at a given time, $t$, or:

$$
w_{\text {max }}=f\left(r_{C}, t\right)
$$

where:

$$
\left(\frac{\sigma w}{\sigma r}\right)_{r_{C}, t}=0 \quad \text { and } \quad \frac{d r_{C}}{d t}=v_{s}
$$

The crest displacement at a given time and at a certain station is not always the absolute maximum that the station may undergo. In fact, because of the "close in", rapid geometrical atcenuation of the waves in the near-anchor region (section 3.3), the absolute maximum aisplacement in the time history of motions of a "close in" station occurs earlier than the passage of the crest of the wave. However, as the wave moves further away and into the free field, the rate of attenuation decreases, the wave front advances as a plane wave and, therefore, the time of maximum displacement in the time history coincides with the time of the passage of the crest of the wave. Fig. 5-6 illustrates that station 5 is the approximate boundary for the zone closer to the anchor where the passage of the crest of the wave does not coincide with the absolute maximum displacement. Station 5 is located approximately 20 inches from the anchor. 
On the particle velocity-time record, the point is also readily identified as the time of second crossing of the main pulse or $t$ " In Fig. $5-4$.

This particular point has been chosen to identify the time for computing the propagation velocity because:

1. It corresponds to a well defined physical characteristic of the wave propagation phenomena.

2. It occurs after the soll has been subjected to maximum strain, therefore being a good indicator of the strain dependent benavior of the material.

3. It can be used in zones closer to the anchor, since It is less 11 kely to be altered, by the primary pulses, than points which precede It, as would be the case of the times of first arrival and positive peak of the main pulse.

\subsubsection{Velocities from Waveforms}

From Figs. 5-6 and 5-7, several complications or limitations appear apparent when using the crest of the waveforms to determine propagation velocity. The spacing of the recelving stations and the time resolution control the precision with which the crest of the wave can be defined. Also the displacements include a component of primary wave which distorts the main wave in the "close in" region. Finally the anchor record cannot be usea to define the waveforms in the "close in' range primarily because it measures anchor movements and slippage at the soll anchor interface as well as motions of the surrounding soll.

In Fig. 5-7 the radial distance is measured from the edge of the anchor. From this figure and Fig. 5-6, the shear 


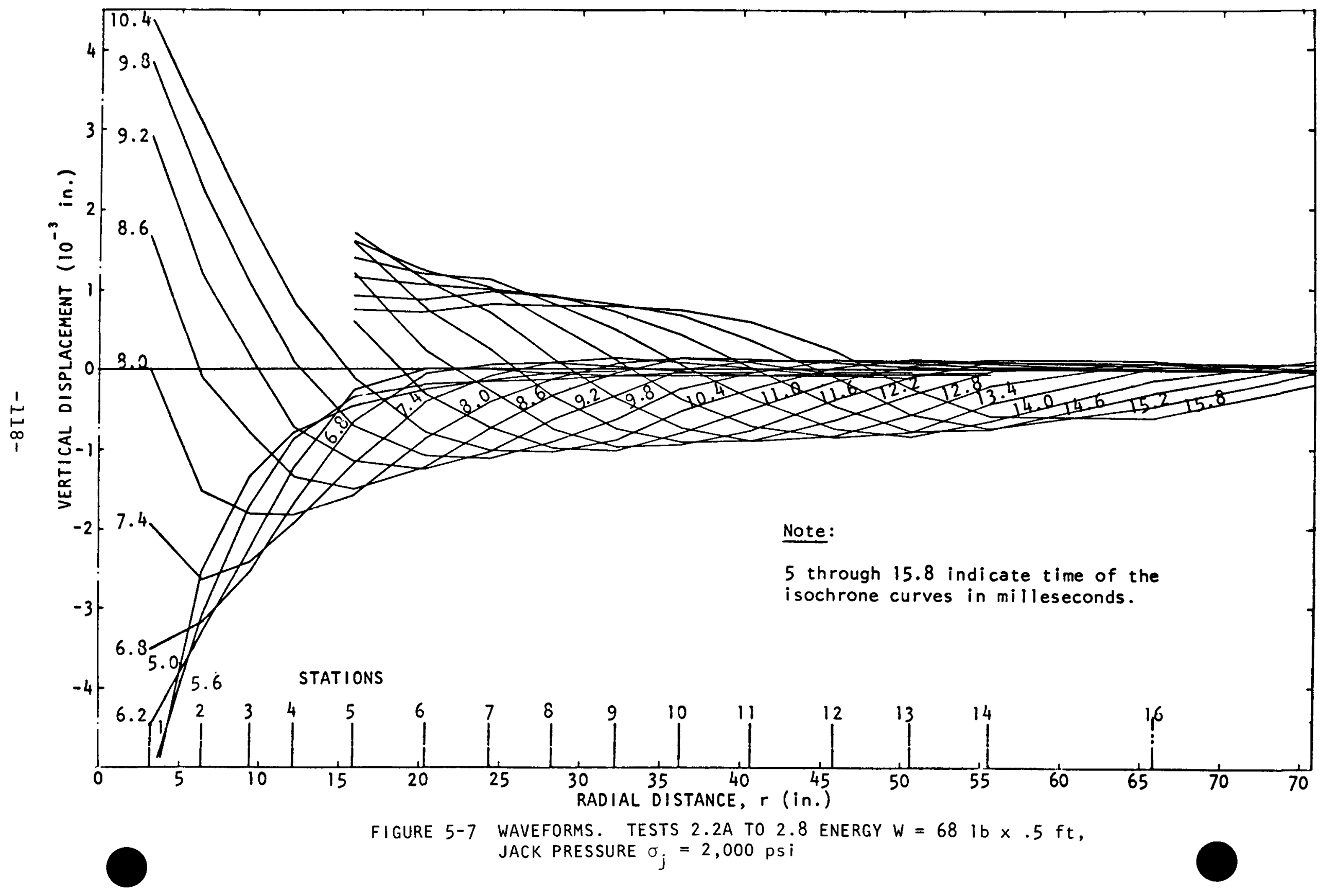


wave velocity has been computed as shown in Tables 5.1 and 5.2 , for the free field zone, between stations 6 and 17 . The point to point computations give results which vary considerably while the average velocities for distances in the order of every 10 inches vary to a lesser extent. The precision of point to point measurements is dependent on the definition of the location of the crest of the wave. Since only a few points, spaced at distances of 4 to 10 inches, are available, this definition of the crest in this region may be inaccurate. Velocities determined in Table 5.1 close to the anchor are generally higher than the rest, which is opposite to the trend of decreasing modulus with increasing strain. This anomaly may be due to the higher confining pressure caused by the effect of the radial stress of the anchor or to the component of primary wave pulses. It may also be due to the presence of a local dense lense of soil or to the inaccuracy of the waveform method near the anchor as indicated by the finite element results (Fig. 4-18). The proximity to the close range zone may also have influenced the first points of the Table 5.1, since parts of the waves are in that range. All other average velocities in these two tables agree with generally accepted soil behavior characteristics (i.e., to decrease in stiffness as the strain level increases).

Finally, it is also pertinent to note that these average velocities agree well quantitatively with the velocities determined from cyclic triaxial tests summarized in Appendix $C$, Fig. C-5. This is especially important when considering that both the in situ mass of soil and the laboratory samsles were prepared as remolded materials.

5.3 .2 Comparison of Several Methods for Determining velocity

For production tests, it is not possible to construct waveform curves, because there is an insufficient number of free field recording stations. However, for the special tests, it is 


\section{Table 5.1}

Shear Wave Velocity

For Waveforms in Fig. 5-7

Location of Time of Travel Travel Crest of Wave $r_{\mathrm{C}}$ (in) Waveform $t \quad$ (msec)
Time $\Delta r$ (in) $\Delta t$ (msec)
Shear Wave Velocity $\frac{V_{s}\left(\frac{\mathrm{ft}}{\mathrm{sec}}\right)}{\substack{\text { Point to Average } \\ \text { Point }}}$

$$
22.8
$$

26.3

30.6

33.6

37.3

41.2

45.0

48.6

52.3

55.5

60.6
9.8

10.4

11.0

11.6

12.2

12.8

13.4

14.0

14.6

15.2

15.8
3.5

4.3

3.0

3.7

3.9

3.8

3.6

3.7

3.2

5.1
.6

.6

.6

.6

.6

.6

.6

.6

.6

.6
436

597

417

514

541

528

500

514

444

708
490

514

542

490

577 


\section{Table 5.2}

\section{Shear Wave Velocity}

From Times $t_{1}$ of Peak Particle Velocity from Fig. 5-3

\begin{tabular}{|c|c|c|c|c|c|}
\hline $\begin{array}{l}\text { Sensor } \\
\text { Station }\end{array}$ & $\begin{array}{c}t_{1} \\
\text { (msec) } \\
\end{array}$ & $\begin{array}{l}\Delta r \\
(i n) \\
\end{array}$ & $\begin{array}{r}\begin{array}{c}\Delta t_{1} \\
(\mathrm{msec})\end{array} \\
\end{array}$ & $\begin{array}{l}\text { Shear Wave } \\
\text { Station to } \\
\text { Station }\end{array}$ & $\frac{\text { Velocity (Eps) }}{\text { Average }}$ \\
\hline 6 & 7.54 & / & & & \\
\hline 7 & 8.34 & 3.94 & .8 & 410 & \\
\hline 8 & 9.1 & 3.94 & .76 & 432 & 421 \\
\hline 9 & 9.68 & 3.87 & .58 & 556 & \\
\hline 10 & 10.42 & 4.06 & .74 & 457 & \\
\hline 11 & 11.12 & 4.32 & .7 & 514 & 509 \\
\hline 12 & 11.98 & 5.12 & .86 & 496 & \\
\hline 13 & 12.68 & 5.0 & .7 & 595 & \\
\hline 14 & 13.38 & 4.38 & .7 & 581 & 557 \\
\hline 16 & 14.62 & 10.37 & 1.24 & 697 & \\
\hline 17 & 15.9 & 9.88 & 1.28 & 643 & 670 \\
\hline
\end{tabular}


possible to use this data as a tool to compare velocities determined from the waveforms with recognizable points on the velocity histories to aid in evaluating procedures for data reduction. Ideally, for the "free field" stations, the crest point generally corresponds to the point of zero crossing ( $t$ " in Fig. 5-4). However, finite element results indicate that the first peak velocity point $\left(t_{1}\right)$ may be an equally acceptable point. Therefore, as a first test, the three most identifiable points on the main velocity time record $\left(t_{1}, t ", t_{2}\right)$ were determined for all stations for a typical test series, the results of which are plotted as Fig. 5-8. The three curves generally parallel each other indicating that any of the three points provide reasonable values of shear wave velocity especially for the more distant sensors. However, comparison of the two lower curves show that the second crossing times, $t "$, provide a smoother curve than the first peak times, $t_{1}$. This is probably because the particle velocity is changing more rapidly at $t$ " and is therefore less affected by minor distortions in the wave shape itself. Also in the "close in" region, the first peak time is probably more affected or contaminated by the separating primary pulse. The smooth curvature of the second crossing points in this region indicate that this time point is less affected by strong primary pulses. Based on this comparison, it therefore appears that the zero crossing is the most identifiable point of the three time points for use in computing shear wave velocities especially for the "close in" region. The third point, $t_{2}$, is often distorted in actual production tests and therefore has received only minimal attention.

Before comparing these time points with the points corresponding to the wave crest, it was considered desirable to refine the waveforms for this example case by removing the primary pulse from each velocity time signature prior to integrating and constructing the waveform curves. Also to provide a clearer definition of the crest of the waves, the displacements 


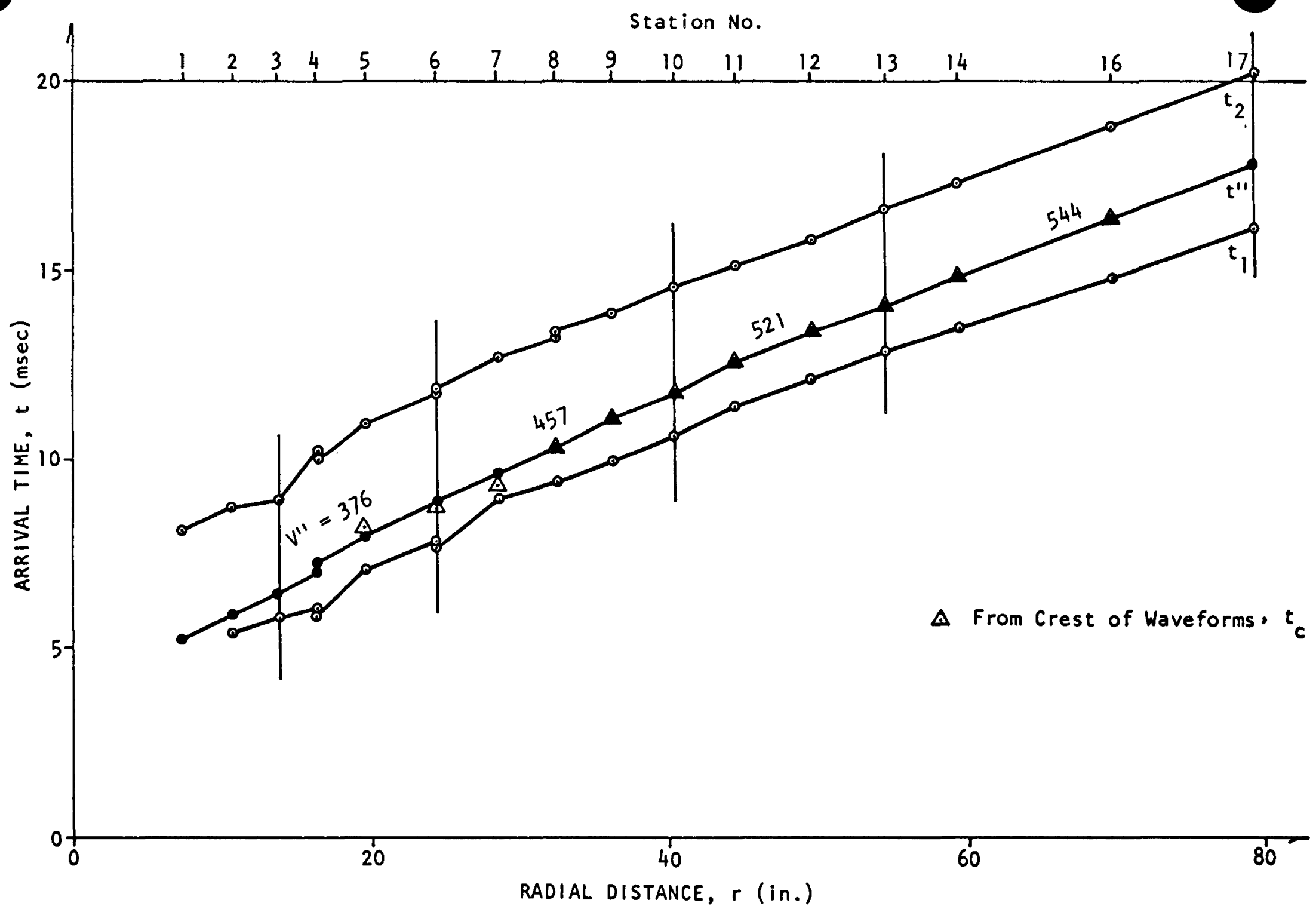

FIGURE 5-8 TIME-DISTANCE PLOTS. TESTS 2.10 TO 2.17A

ENERGY OF IMPACT, $W=58$. I $\times 1 \mathrm{ft}$. JACK PRESSURE, $\sigma_{j}=2,500 \mathrm{ps} i$ 
were greatly amplified. The time points obtained from this refinement of the waveforms have also been superimposed on Fig. 5-8. As indicated in this figure, data in the "close in" range is not included primarily because of the difficulties described earlier in this section and the inaccuracies noted in chapter 4. The excellent agreement between this procedure for the indicated free field range when compared with time points on each velocity record indicate that the point corresponding to the zero crossing is indeed a proper point for identifying the arrival time of the shear wave at free field stations in production testing.

To further study the dependency of measurements of velocity on different energies of impact, three series of tests were performed at a constant jack pressure of 3,500 psi and different energies. A summary of the computed point to point velocities and average velocities using the time of zero crossing $\left(t^{\prime \prime}\right)$ is presented in Table 5.3 .

The point to point data has its normal erratic variations, however the average values indicate reasonable soil behavior characteristics. Not only does the wave velocity increase as the wave propagates outward (i.e., increase in velocity with a decrease in strains) but also for respective stations a reduction in velocities with increasing energies is also evident. This data thus demonstrates in two ways that the shear wave velocity and thus the modulus is not only strain dependent but that this strain dependency can be assessed with in situ measurements.

\subsection{SHEAR STRAINS IN FREE FIELD SOILS}

Methods for the determination of shear strains in the free field have been tested using the analytical test results and are discussed in detail in Chapter 4. Shear strains were 
Table 5.3

Shear Wave Velocity, V" (fps), Versus Energy

\begin{tabular}{|c|c|c|c|c|}
\hline Stations & & $\sqrt{1 b} \times f$ & & \\
\hline No. & $58 \times .5$ & $150 \times .5$ & 58 & $\times 1.5$ \\
\hline$A-1$ & 566 & 350 & & 253 \\
\hline $1-2$ & 832 & 290 & & 443 \\
\hline $2-3$ & 625 & 521 & & 455 \\
\hline $3-4$ & 218 & 390 & & 364 \\
\hline $4-5$ & 352 & 387 & & 274 \\
\hline $5-6$ & 459 & 393 & & 458 \\
\hline $6-7$ & 430 & 443 & & 434 \\
\hline $7-8$ & 442 & 390 & & 410 \\
\hline $8-9$ & 474 & 474 & & 471 \\
\hline $9-10$ & 589 & 521 & & 473 \\
\hline $10-11$ & 505 & 441 & & 500 \\
\hline $11-12$ & 485 & 502 & & 566 \\
\hline $12-13$ & 550 & 514 & & 457 \\
\hline $13-14$ & 535 & 484 & & 500 \\
\hline $14-16$ & 534 & 527 & & 551 \\
\hline $16-17$ & 542 & 521 & & 526 \\
\hline
\end{tabular}

\section{Average V" (fps) from $t-r$ Curves}

\begin{tabular}{c}
$\begin{array}{c}\text { Stations } \\
\text { No. }\end{array}$ \\
\hline $\begin{array}{c}5-5 \\
12 \\
12-17\end{array}$
\end{tabular}

\begin{tabular}{|c|c|c|c|}
\hline $58 \times .5$ & $\begin{array}{l}W(1 \mathrm{~b} \times \mathrm{ft} \\
150 \times .5 \\
\end{array}$ & 58 & $\times \quad 1.5$ \\
\hline 426 & 371 & & 333 \\
\hline 483 & 452 & & 473 \\
\hline 539 & 512 & & 509 \\
\hline
\end{tabular}


computed from the distortion of individual finite elements and then compared with maximum strains determined from the ratio of $\dot{w} / V_{S}$ (Eq. 3.13). The data, Fig. 4-18, showed that shear strains determined from $\dot{w} / V_{S}$ ratio agree well with computed values at distances greater than 20 inches, while for the cases and computational techniques considered in the figure, the "close in" values were about 30 to 50 percent too low. This large variation in the strains from the ratio equation is not surprising, it is known that this equation is not theoretically applicable in the near-anchor region.

Since the finite element calculations do not duplicate all aspects of the "real" in situ conditions, a similar comparison was made with the experimental data. A sample of test data comparing the strains determined from the waveforms (Eq. 5.4) with those calculated from the $\dot{w} / V_{S}$ ratio is presented in Fig. 5-9. This figure shows good agreement and further supports the validity of the analytical calculations and the comparison of this test data. This data further shows that, for the distance range shown, the strains determined from the waveforms agree well with the strains determined from the particle velocity shear wave velocity ratio. This is the same range where the finite element computea strains also agreed well with the strains computed from the velocity ratio. "Close in" strain data is neither shown nor compared because of uncertainties in interpreting the correct particle velocity value for the latter case. The strong primary wave present in this region could greatly influence the value of the peak particle velocity and thus the computed $\dot{w} / V_{S}$ ratio. Approximate strains determined from waveforms from the finite element calculations of chapter 4, in which there were no primary waves, agreed within about 10 percent with the exact strain values computed during the calculations. This arfeement was obtained in spite of the failure of the wave-form methods to provide good estimates of the shear wave velocity in the near-anchor region. 


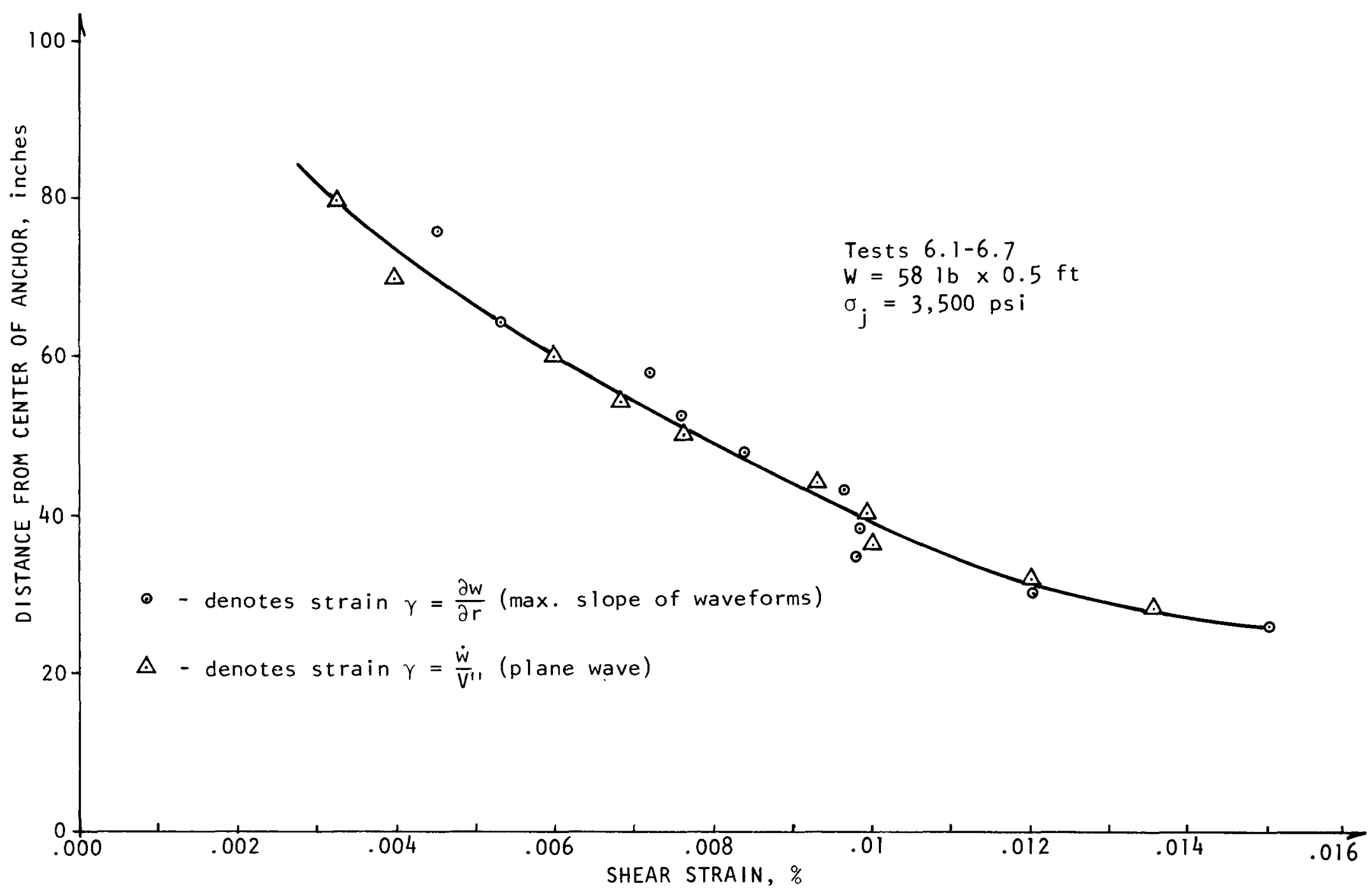

FIGURE 5-9 COMPARISONS OF STRAINS DETERMINED BY DIFFERENT METHODS 
Since the waveforms provide reasonable approximations of the shear strain (but not necessarily wave velocities) in the "close in" region, both the influence of jack pressure and energy level on the strain in this region can be studied more closely. For this assessment four series of tests were compared, three at a constant jack pressure and varying impact energies and one with a variable jack pressure but the same energy as one of the previous three series. The strains computed from these series of tests from constructed waveforms are summarized in rable 5.4. Strain plots of the three series of tests with variable impact energies are presented as Fig. 5-10. The very strong attenuation in strain in the first 18 to 20 inches is clearly evident. The increase in strain with increased energies is also consistent. However, because of the rapid rate of attenuation "close in", actual strain values in the free field under various energy conditions are changed only a small amount and in fact appear only important in the closer range. Since the first free field sensor in production tests is located at an approximate radial distance of 48 inches, it is apparent that significantly larger strains cannot be achieved with larger energies and therefore the use of excessively strong equipinent with very heavy hammers is not warranted for this test.

A comparison of the two series of tests at different jack pressures in Table 5.4 also indicates that increased jack pressures produce larger strains which are only significant in the closer range. This comparison would indicate that variations in jack pressure do not produce significantly different strains in the free field and therefore is not likely to significantly affect measured test values. The effects of jack pressure and energies (hammer weight and height of drop) are treated in greater detail in Appendices $F$ and $G$. 
Table 5.4

Shear Strain, $\gamma(\%)$, Versus Energy and Jack Pressure

\begin{tabular}{|c|c|c|c|c|c|c|c|c|c|c|}
\hline \multirow[b]{2}{*}{$r(\mathrm{in})$} & \multirow[b]{2}{*}{ Sta. \# } & \multicolumn{6}{|c|}{$\begin{array}{l}\sigma_{j}=3,500 \mathrm{psi} \\
W(1 \mathrm{~b} \times \mathrm{ft})\end{array}$} & \multicolumn{3}{|c|}{$\begin{array}{l}\sigma_{j}=2,000 \mathrm{psi} \\
\mathrm{W}(1 \mathrm{~b} \times \mathrm{ft})\end{array}$} \\
\hline & & 58 & $x \quad .5$ & 150 & $\times \quad .5$ & $58 x$ & 21.5 & 58 & $x$ & .5 \\
\hline 5.9 & $A-1$ & 2.16 & $\times 10^{-1}$ & 4.36 & $\times 10^{-1}$ & 6.3 & $\times 10^{-1}$ & & & \\
\hline 9.1 & $1-2$ & 9.7 & $\times 10^{-2}$ & 1.69 & $\times 10^{-1}$ & 1.97 & $\times 10^{-1}$ & 9.2 & $x$ & $10^{-2}$ \\
\hline 12.2 & $2-3$ & 5.9 & $\times 10^{-2}$ & 9.2 & $\times 10^{-2}$ & 1.1 & $\times 10^{-1}$ & 4.6 & $\mathrm{x}$ & $10^{-2}$ \\
\hline 14.9 & $3-4$ & 4.4 & $\times 10^{-2}$ & 8.4 & $\times 10^{-2}$ & 8.5 & $\times 10^{-2}$ & 4.0 & $x$ & $10^{-2}$ \\
\hline 17.1 & $4-5$ & 3.4 & $\times 10^{-2}$ & 5.7 & $x 10^{-2}$ & 6.0 & $\times 10^{-2}$ & 2.96 & $\mathrm{x}$ & $10^{-2}$ \\
\hline 22.2 & $5-6$ & 2.0 & $\times 10^{-2}$ & 3.5 & $\times 10^{-2}$ & 3.1 & $\times 10^{-2}$ & 1.73 & $\mathrm{x}$ & $10^{-2}$ \\
\hline 26.4 & $6-7$ & 1.5 & $\times 10^{-2}$ & 2.8 & $\times 10^{-2}$ & 2.5 & $\times 10^{-2}$ & 1.32 & $x$ & $10^{-2}$ \\
\hline 30.5 & $7-8$ & 1. 2 & $\times 10^{-2}$ & 2.2 & $\times 10^{-2}$ & 2.0 & $\times 10^{-2}$ & 1.09 & $\mathrm{x}$ & $10^{-2}$ \\
\hline 34.4 & $8-9$ & 9.8 & $\times 10^{-3}$ & 1.5 & $\times 10^{-2}$ & 1.6 & $\times 10^{-2}$ & 8.01 & $x$ & $10^{-3}$ \\
\hline 38.5 & $9-10$ & 9.9 & $\times 10^{-3}$ & 1.44 & $\times 10^{-2}$ & 1.4 & $\times 10^{-2}$ & 9.41 & $\mathrm{x}$ & $10^{-3}$ \\
\hline 43.0 & $10-11$ & 9.7 & $\times 10^{-3}$ & 1.45 & $\times 10^{-2}$ & 1.2 & $\times 10^{-2}$ & 7.75 & $\mathrm{x}$ & $10^{-3}$ \\
\hline 48.0 & $11-12$ & 8.4 & $\times 10^{-3}$ & 1.2 & $x 10^{-2}$ & 1.1 & $\times 10^{-2}$ & 7.62 & $\mathrm{x}$ & $10^{-3}$ \\
\hline 53.0 & $12-13$ & 7.6 & $\times 10^{-3}$ & 1.1 & $\times 10^{-2}$ & 1.06 & $\times 10^{-2}$ & 6.4 & $x$ & $10^{-3}$ \\
\hline 58.0 & $13-14$ & 7.2 & $\times 10^{-3}$ & 8.6 & $\times 10^{-3}$ & 9.0 & $\times 10^{-3}$ & 7.58 & $x$ & $10^{-3}$ \\
\hline 65.3 & $14-16$ & 5.3 & $\times 10^{-3}$ & 7.4 & $\times 10^{-3}$ & 7.0 & $\times 10^{-3}$ & 5.11 & $x$ & $10^{-3}$ \\
\hline 75.6 & $16-17$ & 4.5 & $\times 10^{-3}$ & 6.2 & $\times 10^{-3}$ & 6.0 & $\times 10^{-3}$ & 4.45 & $\mathrm{x}$ & $10^{-3}$ \\
\hline
\end{tabular}




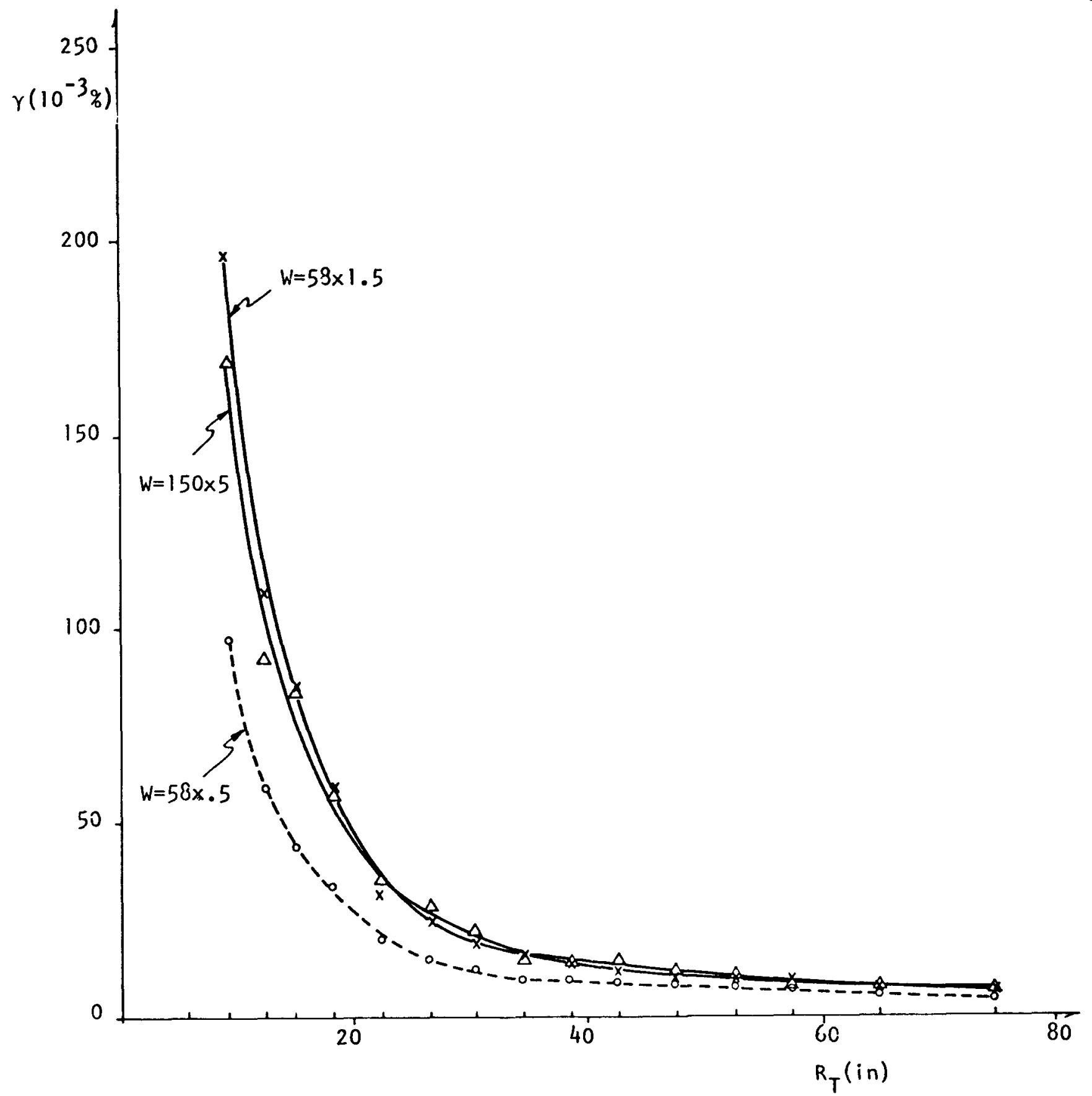

FIGURE 5-10 SHEAR STRAIN FROM WAVEFORMS

VERSUS RADIAL DISTANCE $\sigma_{j}=3,500 \mathrm{psi}$ 


\subsection{SHEAR WAVE VELOCITY AND STRAINS IN SOILS}

ADJACENT TO THE ANCHOR

The in situ impulse test provides accurate measurements for the determination of shear moduli and shear strains in the free field. The region adjacent to the anchor cannot be incorporated in these measurements because of the special conditions in the surroundings of the anchor source. However, approximate values of wave velocities and shear strains could be obtained in this region, if the records of loads and motions of the anchor could be used to estimate the response of the adjacent soils. In order to investigate the possibilities of this approach, under the controlled conditions in the compacted fill, detailed measurements of anchor loads and motions were recorded in different special tests. This enabled the "real" test conditions to be studied in relation to the more simplified theoretical models defined in chapters 3 and 4 . As described in Appendix D, the anchor was instrumented with a load cell to measure the force applied to the anchor, and several velocity transducers and accelerometers to measure anchor motion characteristics. Simultancous measurements of these characteristics together with adjacent sensors in the soil would provide sufficient information to obtain the shear wave velocity and the corresponding strain at the anchor.

\section{$5.5 .1 \cdot$ Anchor Records}

A sample of records made from transducers on the anchor and in the adjacent sensor are presented in Fig. 5-11 to illustrate characteristic shapes and points of each time history. The applied load pulse has a slightly irregular triangular or parabolic shape while the acceleration is a very random exratic series of spikes. The latter two velocity curves cxcept for differences in amplitude in general exhibit amazingly similar shapes. The three anchor measurements, although possessing a 


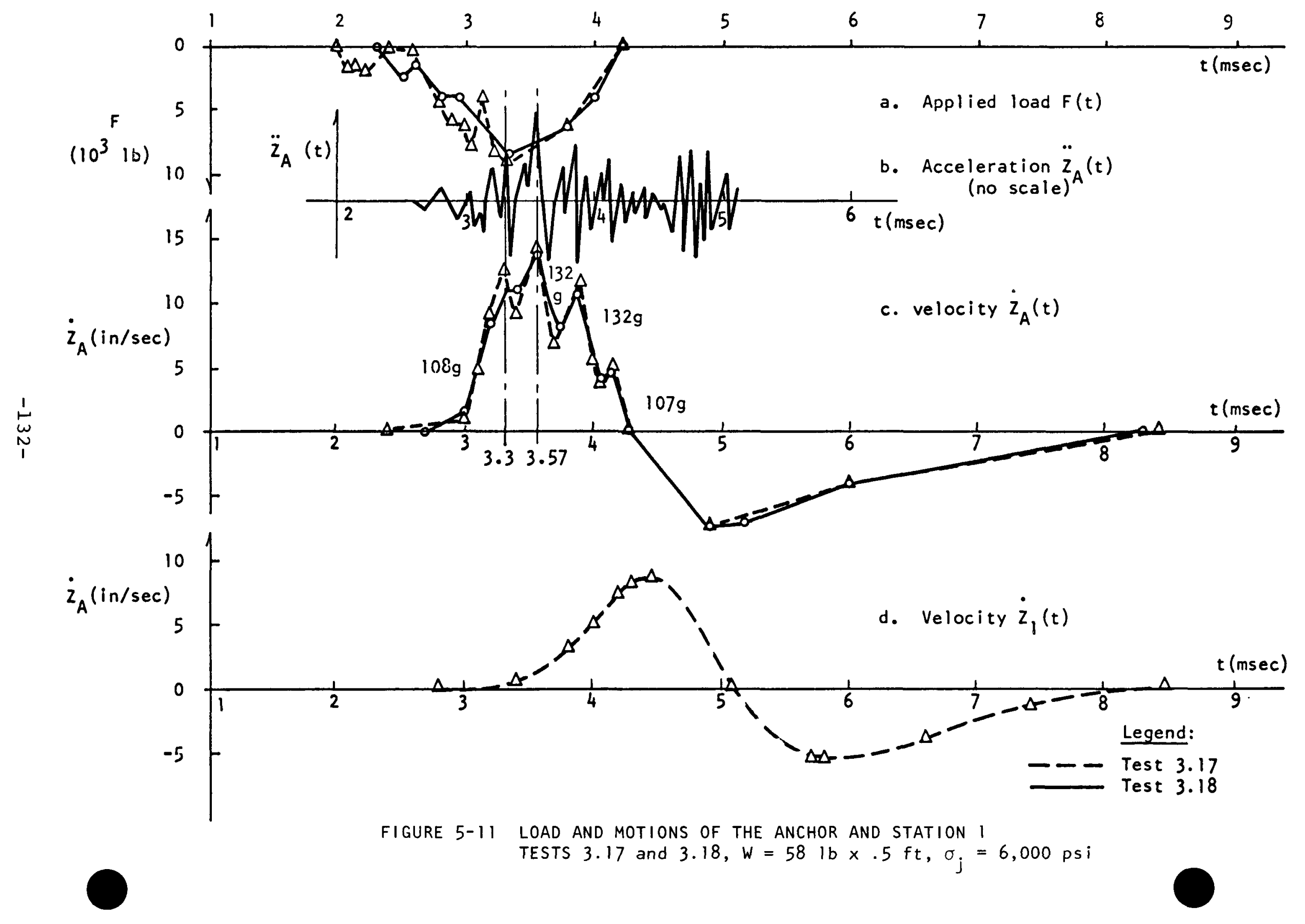


wide variety of shapes, all show a common time referring to the start of the event (or impact). This characteristic time is a lower bound value and if plotted on a time distance graph with free field points as in Fig. 2-l defines a slope corresponding to a lower bound value of shear wave velocity. While this point does not define a true shear wave velocity in the soil, it provides a reference lower bound value at the location of the anchor.

The force time history was used primarily in this experimental study as a base for correlating the effect of jack pressure and for studying force characteristics at the adjacent soil anchor interface. For the range of jack pressures, hammer weights and drop heights used in these tests, force values of the order of 5,000 to 30,000 pounds were generally obtainea. Force shape, magnitude, and signal duration, and rise time characteristics are discussed and related to jack pressure in Appendix G. These measurements and a comparison of various relationships in this appendix demonstrate the importance of achieving optimum anchor coupling.

Measurements of acceleration in the anchor were limited to one transuucer, as the lower unit became damaged and did not operate. The data from this source was somewhat limited primarily because the measured motion was a registration of the passes of stress waves traveling up and down the high velocity metals of the anchor (i.e., propagation velocity of aluminum is $16,000 \mathrm{ft} / \mathrm{sec}$ ). The records obtained were therefore a superposition of high frequency pulses which represent the stress changes in an element of a plate on the anchor and not entirely the motion of the anchor as a whole. These high frequency measurements, in general, masked out the lower frequency motions typical of the total motion of the anchor. The accelerometers were placed such that $R_{s}$ (the resisting force in the soil Eq. 3.12) could be computed from direct measurements. One other 
less direct approach for estimating the acceleration of the anchor is from the slope of the velocity time history. Minor time shifts between the different recording transducers, however, dia not produce any seemingly meaningful relationships. These relationships will be studied further in future efforts described in Chapter 6 .

Particle velocity records of the anchor and records several inches away in the surrounding soil enabled relative transfer motion characteristics across the soil anchor interface to also be studied. As indicated in the Fig. 5-11, the shapes of the two curves were generally quite similar when excessive slippage did not occur. As the wave passed between these two stations, there was an attenuation in signal amplitude and a general filtering of the high frequency components in the anchor record. Where slippage was excessive, the anchor record became greatly distorted primarily because the input motion to the anchor exceeded the capacity of the transducer to record it correctly.

While the measured motion and force characteristics provided little additional theoretical understanding of the test phenomenology, they nevertheless provided additional definition of magnitude and forces acting on the anchor and transfer and stress characteristics (slippage and coupling) at the anchor soil interface. The data also provided consistent time and signal amplitude on the anchor records, to enable both the shear wave velocity and its strain to be estimated using characteristic points.

The approach for data interpretation of the anchor record was thus one of comparing free field velocities and strains determined from tre experimental tests with those estimated in the anchor region. Once this was accomplished with the closer spaced special test measurements, empirical 
methods could be developed for use with production test data.

\subsubsection{Dimensional Analysis Procedures}

Dimensional analysis procedures were used to find a relationship between the main variables of the test. The dimensional analysis procedure permits definition of dimensionless products formed by the variables of the test. These variables are:

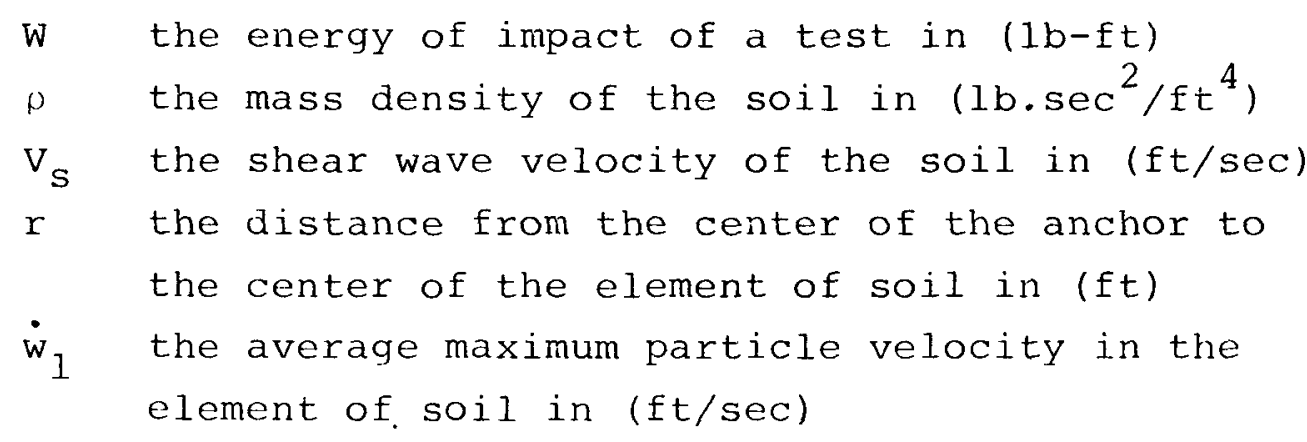

Dimensionless products can then be related to represent a physical equation as $\phi\left(\eta_{1}, \pi_{2}\right)=0$ as long as all of the variables are included. Convenient dimensionless products for this equation are:

$$
\begin{aligned}
& { }_{1}=\frac{\dot{w}_{1}}{\mathrm{v}_{S}} \\
& n_{2}=\frac{\left(f \mathrm{v}_{S}{ }^{2}\right)^{\frac{1}{3}} r}{w^{\frac{1}{3}}}
\end{aligned}
$$

where $\rho \mathrm{V}_{\mathrm{S}}{ }^{2}=\mathrm{G}$, especially since "1 represents the equation for strain $r$ of plane waves and ${ }_{2} 2$ is an indication of modulus. 
Fig. 5-12 is the dimensional analysis log-log plot of the dimensionless parameters for three series of tests including only the free field stations. These tests were performed with the same jack pressure and different hamner weights and heights of drop. The narrow band of data from this plot shows that for these soils, there is a unique relationship between the variables of the test. Relationships of this type may be usea to estimate the variation of strain with distance from the source. If the natural stress conditions were the same in the close range, as in the free field, the free field relationship could be extrapolated to verify strain values close to the anchor. Unfortunately, the static radial stresses, applied by coupling of the anchor, produce changes in the state of stress, which are difficult to evaluate. Nevertheless, these relationships may be used to verify measurements resulting from impuise tests.

\subsubsection{Shear Wave Velocity at Anchor}

Shear wave velocities in the close range are difficult to estimate because of superposition of pulses from $\mathrm{P}$ and $\mathrm{S}$ waves and differences in states of stress around the anchor. Characteristic time-distance curves, obtained from a complete series of tests, are shown on Fig. 5-13. These curves are formed using times of maximum displacement, $t$ ", and maximum particle velocity, $t_{1}$, for all stations including the anchor record. In the upper curve formed by using the $t$ " times, it is observed that, while free field points plot in fairly well defined curves, close range points are erratic, because of the reasons mentioned above.

Average wave velocities, estimated as average slopes of the time distance curves, are 483 fps between stations 5 and 12, and 539 fps between station's 12 and 17. An approximate upper value of wave velocity between the anchor and station 5 is 


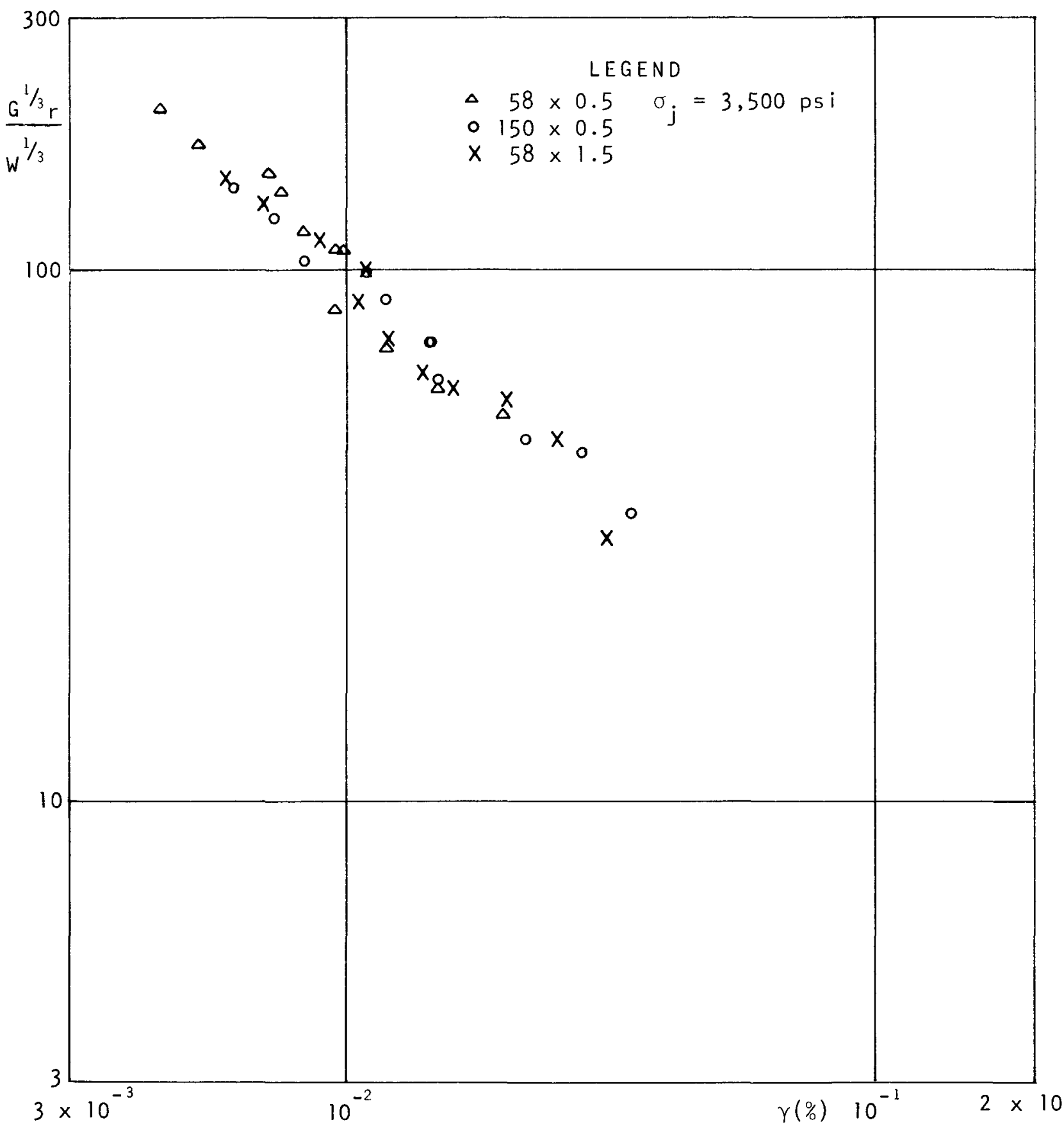

FIGURE 5-12 SOIL MODULUS VERSUS SHEAR STRAIN 


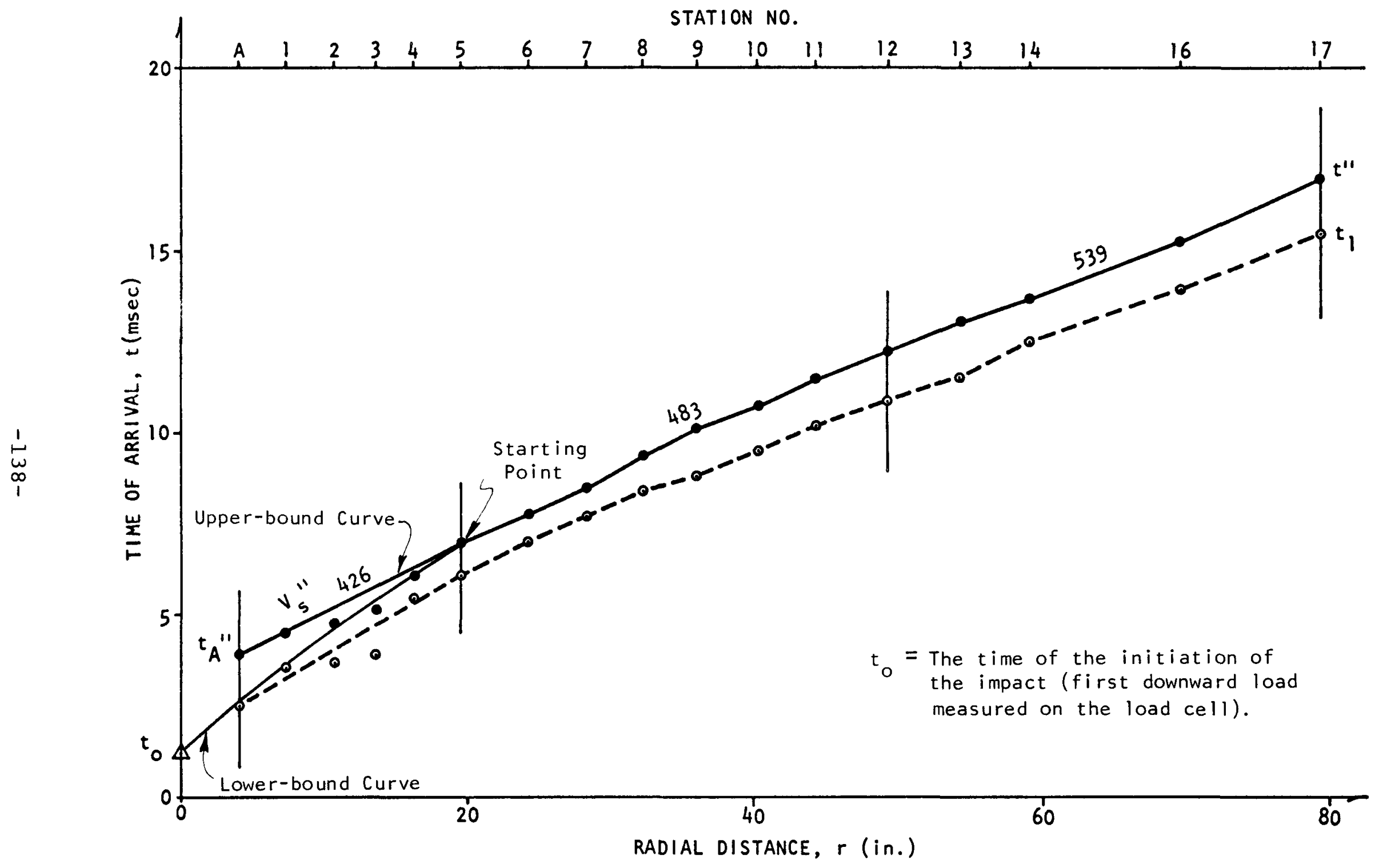

FIGURE 5-13 TIME-DISTANCE PLOTS. TESTS 6.1 TO 6.7

ENERGY OF IMPACT, $W=58 \mathrm{lb} \times .5 \mathrm{ft}$. JACK PRESSURE, $\sigma_{\mathbf{j}}=3,500 \mathrm{ps} \mathrm{i}$ 
given by the slope of the straight line connecting times of maximum displacements of these two stations. This velocity, which is about $426 \mathrm{fps}$ in this case, should be an upper bound value because slippage and higher stresses should delay the occurrence of maximum displacement of the anchor.

In the in situ test, described in Chapter 2, the record of the ancior is different from the records of the free field in that it may include slippage, it represents the superposition of pulses traveling through different paths in the mechanical system and it is part of the forcing functions that generate the waves. From the analyses of this and other tests, in the controlled conditions of the artificial fill, it seems that when firm coupling is achieved, the point of zero crossing, $t$ ", of the anchor record is a consistent upper bound point which relates well with the same points of the records of the free field. However, to use this point from the anchor record to obtain a corrected wave velocity at this point (rather than an upper value), the resultint velocity must be adjusted such that this point corresponds to stress conditions similar to those in the free field. This adjustment becomes especially significant when the applied radial stress is considerably different from the natural state of stress. ihe following correction may be used for this effect. Based on numerous laboratory tests on different types of soils (SW-AA, 1972), the shear modulus, $G$, has been found to be approximately proportional to the square root of the mean effective principal stress, $\bar{\sigma}_{m}$, or the shear wave velocity is proportional to the one-fourth power of the effective principal stress:

$$
\mathrm{V}_{\mathrm{S}}=\mathrm{K}_{1} \bar{\sigma}_{\mathrm{m}}^{\frac{1}{4}}
$$

If $\bar{\sigma}_{m}$ is the mean principal stress in the free field and $\bar{\sigma}_{A}$ is the mean principal stress in the close range, a corrected shear wave velocity at. the anchor or near anchor region can then be obtained from: 


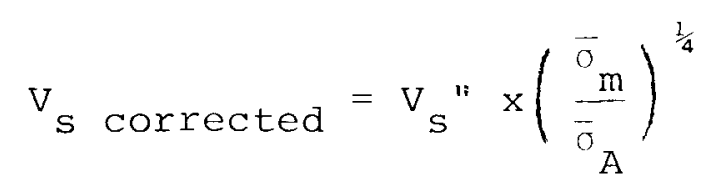

where $V_{s} "$ is the shear wave velocity determined from the time distance curve using times $t$ " or times of maximum displacement.

Other defects incorporated within the anchor records, such as slippage and superposition of pulses cannot be readily assessed either quantitatively or qualitatively. As a result the corrected velocity, adjusted for different stress conditions must be considered as, at best, an estimate of the true shear wave velocity in this region.

\section{$5.5 .4 \quad$ Shear Strain at Anchor}

The shear strain at the anchor has to be estimated somewhat arbitrarily since it is not possible to use the formula:

$$
\gamma=\frac{\dot{w}}{\bar{V}^{\prime \prime}}
$$

because of the practical limitations of the record of the anchor and the theoretical Iimitations of the formula. Finite element results in general indicate that this ratio produces strains in the close in region which are low by 30 to 50 percent. However, this analysis does not include slippage and many of the other factors inherent in the "real" test. These factors, particularly slippage, tend to produce excessive particle velocities at the anchor. These higher values when used in the ratio, tend to compensate for this underestimate of strain.

The use of this ratio together with extrapolation of free field data to the higher range using dimensionless analysis as a secona check both provide reasonable estimates of the shear strain at the anchor. In general, reasonable shear strain values 
determined using similar equipment should produce shear strain values of the order of $10^{-1}$ percent or greater. 
Section 2.4 describes in general terms the data handling and graphical construction procedures for processing production data. The desired parameters derived from this test at a given depth are four shear wave velocities (or modulus) at four different strain levels. To obtain these values, rational procedures had to be established for determining pertinent arrival times from this data to compute propagation velocities. Also practical methods for determining strain had to be provided both for sensors positioned in the free field as well as at the anchor. The experimental and analytical programs described in Chapters 4 and 5 treat this problem in considerable detail. From these efforts, the following procedures are recommended for interpreting these points and completing the production test reduction procedures.

\subsection{FREE-FIELD STATIONS}

Special tests and analytical support calculations show that beyond twenty inches from the anchor, the shear wave travels closely as a plane wave. Since the first station is generally 48 inches away, it and the more distant ones may be treated the same. Arrival times are obtained by selecting common points on each signature provided these points occur after the maximum strain is reached. Maximum strain usually occurs at a time corresponding approximately to the first positive peak on the particle velocity records. Therefore either the time of the first positive peak or the next point of zero velocity crossing should be selected. Of these two points, the crossing point is recommended, because it occurs when the velocity is changing rapidly and is more readily identified. The first velocity peak point while more theoretically applicable is occasionally difficult to define precisely because the rate of velocity change 
(acceleration) is small and therefore subject to minor distortions caused by imperfections in the signature. Also the zero crossing point corresponds to the time of peak displacement (i.e. the crest of the outward propagating wave). Close-in this is not true. The zero crossing/fit method in Chapter 4 is therefore recommended for determining shear wave velocities in the free field.

Shear strains can also be computed assuming that the propagating wave is a plane shear wave using the peak velocity/fit method (i.e. the shear strain = particle velocity amplitude divided by the shear wave velocity). Both theoretical and analytical data have shown that the error involved in such an assumption is of the order of less than about 10 percent for a distance beyond twenty inches from the anchor.

\subsection{ANCHOR STATION}

Within about twenty inches of the anchor, the horizontally propagating shear wave is ideally spherical or cylindrical in nature. Therefore, plane wave theory no longer applies, and treatment of close-in points involves more complex relationships. Fortunately, the only recording station within this range corresponds to the transducer mounted on the plate of the anchor.

When considering the use of corresponding points on this signature with the free field data, the problem becomes greatly complicated. Not only does the close-in soil region propagate waves as cylindrical waves rather than plane waves, but the actual measurement represents more nearly the motion of the anchor as well as slip at the interface. Therefore to theoretically treat this sensor and the record as a free field soil measurement, we not only must consider these factors but also must account for: 
1) stress relief around the borehole occurring when the hole is made,

2) disturbance caused by drilling operations,

3) changes in stress by the application of radial jacking forces to achieve coupling.

Further, the anchor itself is not a rigid body and therefore the recorded motion represents the real motion at only one point on this complex mechanical structure. Thus the record corresponds to a composite train of waves traveling through the complex anchor. It is surprising, when considering all these factors, that this signature so closely resembles those obtained in the free field.

The only way to account for these factors was to perform "close in" special tests under varying conditions and analytical studies and together assess the problem empirically. The procedure consisted of bracketing the shear wave velocity making it representative of an average zone of soil within 18 to 20 inches of the anchor. From the experimental tests, if the same point (specifically the point of second zero crossing) is selected on the anchor signature as the free field signatures, an upper bound value of time and shear velocity is obtained. This estinated upper bound value may be modified to take into account the different state of stress adjacent to the anchor. However, the actual field installation conditions which may occur in an in situ test have to be considered with first priority in the interpretation of test results. Because of these problems, no standard production procedure for use of the records of the anchor can be recommended. The different approaches presented in Chapter 5 may be used to obtain approximate values of wave velocity and shear strain in this region, but they have to be applied in close connection with field observations obtained during the performance of the test. 


\section{3 FUTURE WORK}

As a result of "close-in" experimental special testing and subsequent analytical calculations, much data has been collected, evaluated and has formed the basis upon which the in situ impulse test is founded. Now that procedures are developed, partial future work will consist of actually performing this test on a production basis. This work has been started by testing at accelerograph station sites to evaluate the geotechnical properties including the variation in modulus with strain. This work is planned to continue and will ultimately provide designers of nuclear plants with better geotechnical properties for establishing seismic design criteria.

Most research efforts in the past have been directed toward evaluating the shear modulus variation with shear strain. Future efforts will consist of utilizing this same experimental and analytical test data to evaluate other in situ soil properties such as material damping, P-wave velocities and possibly Poisson's ratio. If additional soil property data can be obtained using the same equipment, the flexibility of the test would be greatly increased.

Initial future efforts will include a thorough evaluation of material damping characteristics. Studies of existing close-in data reported herein including 1 ) attenuation rates from both experimental and analytical results, and 2) isolated anchor force and motion characteristics. From this study, potential approaches or the feasibility for estimating material damping using the in situ procedures and/or equipment will be evaluated. If in situ procedures for even estimating crudely the variation in material damping with strain could be developed, the state of the art of in situ testing for evaluating earthquake engineering problems would be greatly advanced. 


\section{REFERENCES}

Mow, C. C. (1965)

"Transient Response of a Rigid Spherical Inclusion in an Elastic Medium," Jour. Appl. Mech., September, p. 637-642.

SW-AJA (1972)

"Soil Behavior Under Earthquake Loading Conditions: State of the Art, Evaluation of Soil Characteristics for Seismic Response Analyses," A Joint Venture of Shannon \& Wilson, Inc., and Agbabian-Jacobsen Associates, Jan .

SW-AA (1973)

"Soil Behavior Under Earthquake Loading Conditions: In Situ Impulse Technique for Determination of Soil Shear Modulus," A Joint Venture of Shannon \& Wilson, Inc., and Agbabian Associates, June.

SW-AA (1974)

"Soil Behavior Under Earthquake Loading Conditions:

In Situ Impulse Test for Determination of Shear Modulus for Seismic Response Analyses, Progress Report," A Joint Venture of Shannon \& Wilson, Inc., and Agbabian Associates, June.

Terzaghi, K. (1943) Theoretical Soil Iechanics, 4 th ed., John Wiley \& Sons, Inc., New York.

T'roncoso, J. H. (1975)

"In Situ Impulse Test for Determination of Soil Shear Modulus as a Function of Strain," Ph.D. Thesis, University of Illinois.

Westergaard, H. M. (1940)

"Plastic State of Stress Around a Deep Well," Boston Society of Civil Engineers, Vol. 27, January. 
APPENDIX A

DESCRIPTION OF NONLINEAR SOIL MODEL 


\section{APPENDIX A}

DESCRIPIION OF NONLINEAR SOIL MODEL

A mathematical model has been developed which is intended to represent the hysteretic shear stress vs. shear strain behavior of sand. The raw data from which this model was constructed consists primarily of the cyclic triaxial tests summarized in Appendix C. In addition, propagation velocities were available from in situ test data.

The model consists of a variable bulk modulus which depends on $J_{1}$, the first invariant of the stress tensor.

$$
\mathrm{J}_{1}=\sigma_{1}+\sigma_{2}+\sigma_{3}
$$

where $\sigma_{1}, \sigma_{2}, \sigma_{3}$ are the normal stresses at a point in the soil. The sign convention for normal stresses is positive for tension, negative for compression. In addition, the shear modulus varies as a function of both $\mathrm{J}_{1}$ and ' $\mathrm{p}$ ' the principal-shear-stress acting on an element of soil, where

$$
\tau_{\mathrm{p}}=\frac{1}{2}\left[\left(\sigma_{1}-\sigma_{2}\right)^{2}+(2 \iota)^{2}\right]^{\frac{1}{2}}
$$

and $\tau$ is the shear component of stress with respect to the global coordinate axes.

The formula for computing bulk modulus, $K\left(J_{1}\right)$, is

$$
K\left(J_{1}\right)=K_{1}\left[1-K_{2} \exp \left(K_{3} J_{1}\right) \mid\right.
$$

where $K_{1}$, $K_{2}$, and $K_{3}$ are parameters to be chosen to $f i t$ the available data. Eq. A.3 holds regardless of whether virgin loading, unloading, or reloading is taking place. This model, therefore, shows no hysteresis for cyclic loading in bulk. 
However, since constitutive relations in shear are independent of bulk modulus, and since the inelastic finite element calculation consists primarily of shear loading, it is not particularly important to model completely the material behavior in bulk.

The variable shear modulus is expressed in terms of Poisson's ratio which, in conjunction with the bulk modulus, defines the shear modulus. Alternatively, one could have defined the variable shear modulus directly, ignoring any variation which might have occurred in Poisson's ratio. The objective is, however, that the shear modulus be represented realistically; and through Poisson's ratio, a realistic definition of the variable shear modulus is obtained. Therefore, the variablePoisson's-ratio approach is used solely as a device to obtain shear behavior representative of the sand materials for which the triaxial measurements were obtained.

For virgin loading, Poisson's ratio is computed according to the following formula:

$$
v_{V}\left(J_{I}, \tau_{p}\right)=v_{E L}+\left(v_{P L}-v_{E L}\right)\left[1-\exp \frac{\alpha \tau}{J_{1}}\right]
$$

where ' ${ }_{E L}$ ' ' ${ }_{\mathrm{PL}}$ ' and $\alpha$ are parameters to be chosen such that the material behavior agrees with the triaxial test data. Virgin loading occurs whenever $\tau_{p}$ exceeds all previously obtained values of ' $\tau_{p}$. When $\tau_{p}$ is less than the previous maximum value of principal-shear-stress, ' max' the unloading/reloading formula for Poisson's ratio applies.

$$
\nu_{u}\left(J_{1}, \tau_{p}, \tau_{O}, \tau_{\max }\right)=\nu_{E L}+\left[\nu_{v}\left(J_{1},{ }^{\tau}{ }_{\max }\right)-\nu_{E L}\right] T^{\beta}
$$


where $\beta$ is a parameter to be chosen such that the triaxial data is satisfied and

$$
\tau=\frac{\left|\tau_{p}-\tau_{o}\right|}{\left|\tau_{o}\right|^{+}+\tau_{\max } T}
$$

The function $v_{V}\left(J_{I}, \tau_{\max }\right)$ in $\mathrm{Eq}$. A.5 is the virgin Poisson's ratio, defined by Eq. A.4. In Eqs. A.5 and A.6, 'o is the value of the most recent relative-maximum principal-shear-stress. A sign is assigned to $\tau_{0}$ based on the criterion of whether or not the plane on which $\tau_{p}$ acts in a positive sense has rotated more than $45^{\circ}$ from the plane on which $\tau_{0}$ acted in a positive sense. If $\tau_{p}$ and 'o are opposite in sign, the unloading-Poisson's-ratio is prevented from exceeding the virgin Poisson's ratio which would exist for the same value of principal-shear-stress. This prevents the possibility of an element possessing a higher shear modulus in its second cycle of loading than it had in its first cycle under the same state of stress. Once the stressdependent Poisson's ratio has been computed, the corresponding stress-dependent shear modulus is obtained by the following formula:

$$
G=\frac{3 K(1-2 v)}{2(1+v)}
$$

where $G$ is the shear modulus, $K$ is the bulk modulus, and $v$ is Poisson's ratio.

The parameters chosen for the representation of the sand used in the special tests are listed in Table A-1. 


$$
\begin{array}{rlrl}
\mathrm{K}_{1}=50,000 \mathrm{psi} & v_{\mathrm{EL}} & =0.34 \\
\mathrm{~K}_{2}=0.98 & v_{\mathrm{PL}} & =0.48 \\
\mathrm{~K}_{3}=0.019 & \alpha & =10 \\
B & =0.5
\end{array}
$$

\section{A-1 COMPARISON WITH CYCLIC TRIAXIAL TEST DATA}

Two series of cyclic triaxial tests were carried out using the sand from the special tests. The triaxial tests consisted of cyclic loading and unloading at various strain levels with a constant confining pressure. A confining pressure of 6 psi was used in the first series of tests, while 18 psi was used in the second series. The finite element sand model was subjected to load histories intended to simulate these two test series. Comparisons of the finite element stress/strain paths with those of the triaxial tests are presented in Figs. A-l and A-2.

Fig. A-l shows the comparison between model behavior and triaxial data at a confining pressure of 6 psi. The model was subjected to a single load cycle corresponding to the loading of Test 1-B', followed by a portion of a load cycle corresponding to Test 1-C'. It should be noted from Fig. A-la that for any value of stress, the slope of the stress/strain path for the first cycle of loading of the finite element model is approximately equal to the corresponding slope of the stress/strain path of Test 1-B', Cycle 2. This indicates that Young's modulus, as a function of stress, is modeled correctly at the stress level under consideration. In addition, the area contained in the hysteresis loop appears to be the same both for the model and the triaxial data. 


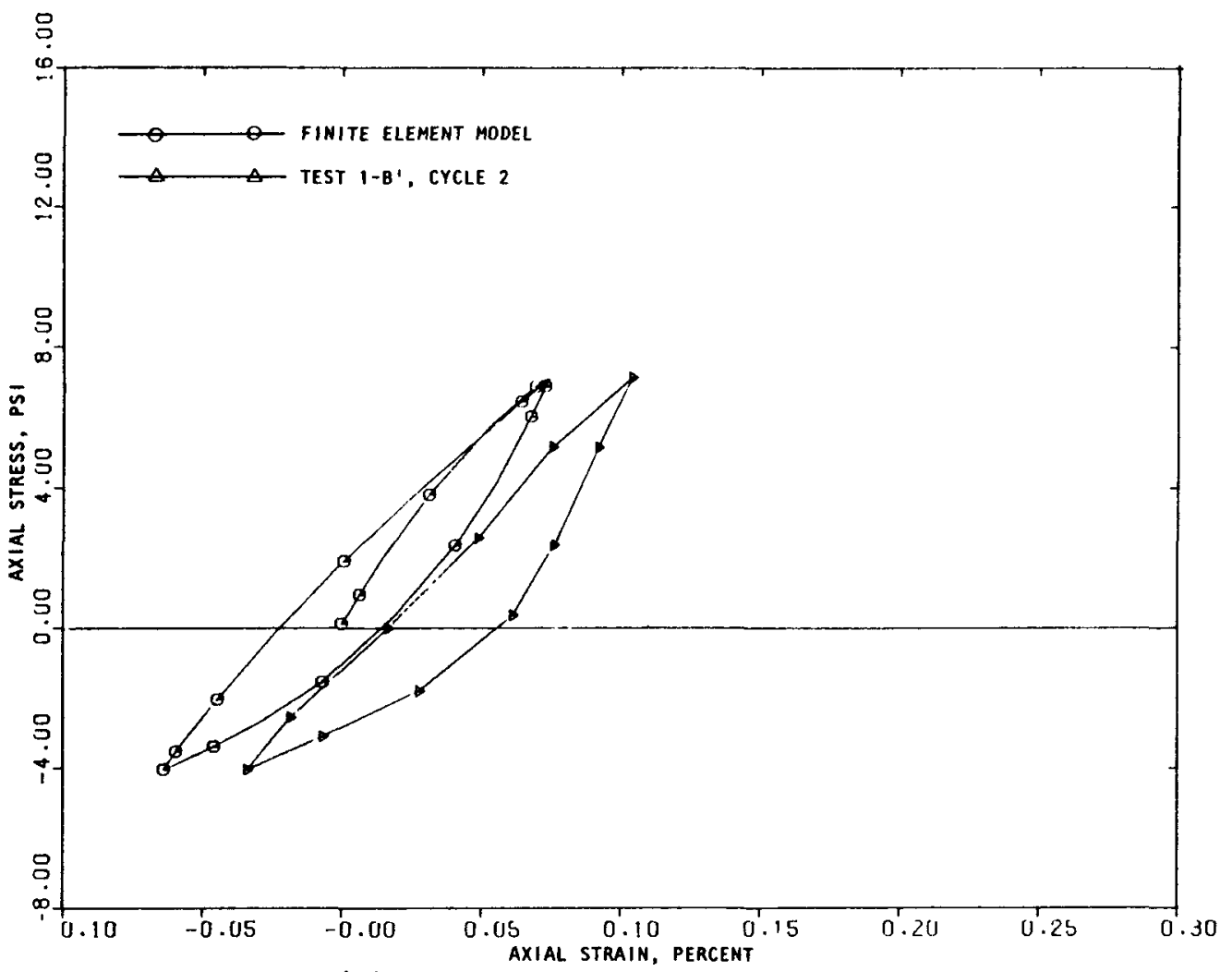

(a) Comparison with Test 1-B'

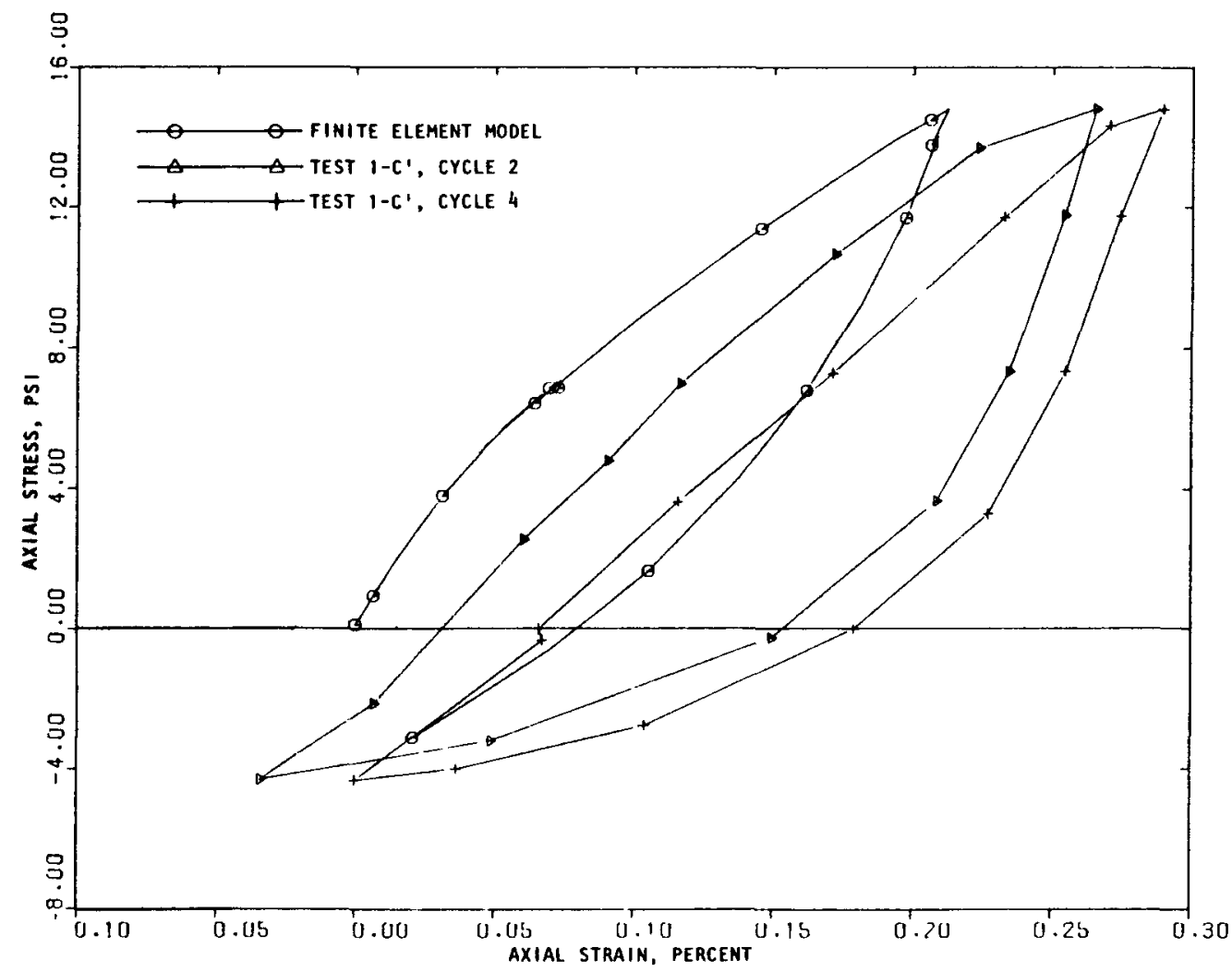

(b) Comparison with Test 1-C'

FIGURE $\bar{A}-1$. COMPARISON OF FINITE ELEMENT MODEL WITH TRIAXIAL DATA AT 6 PSI CONFINING PRESSURE 


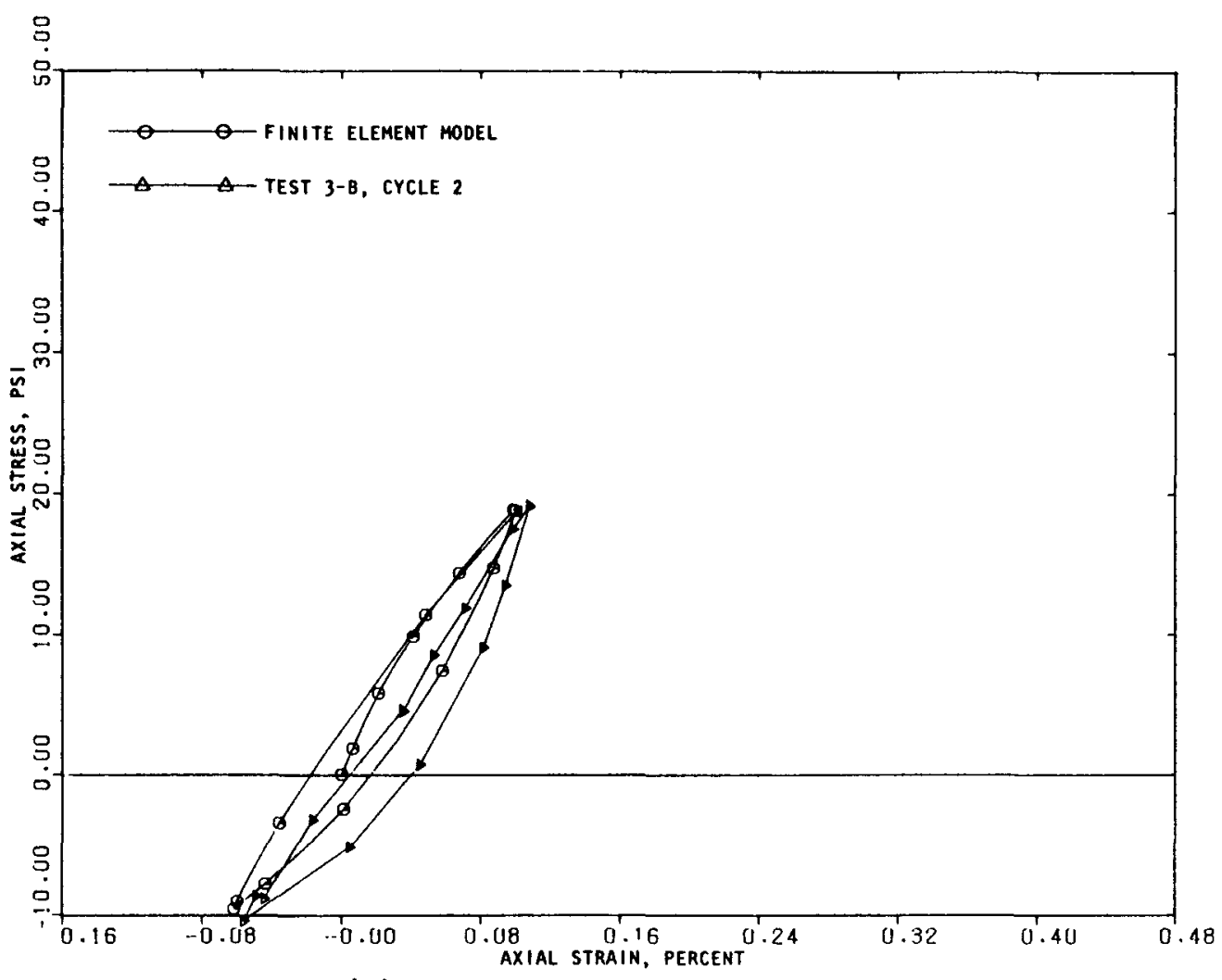

(a) Comparison with Test 3-B

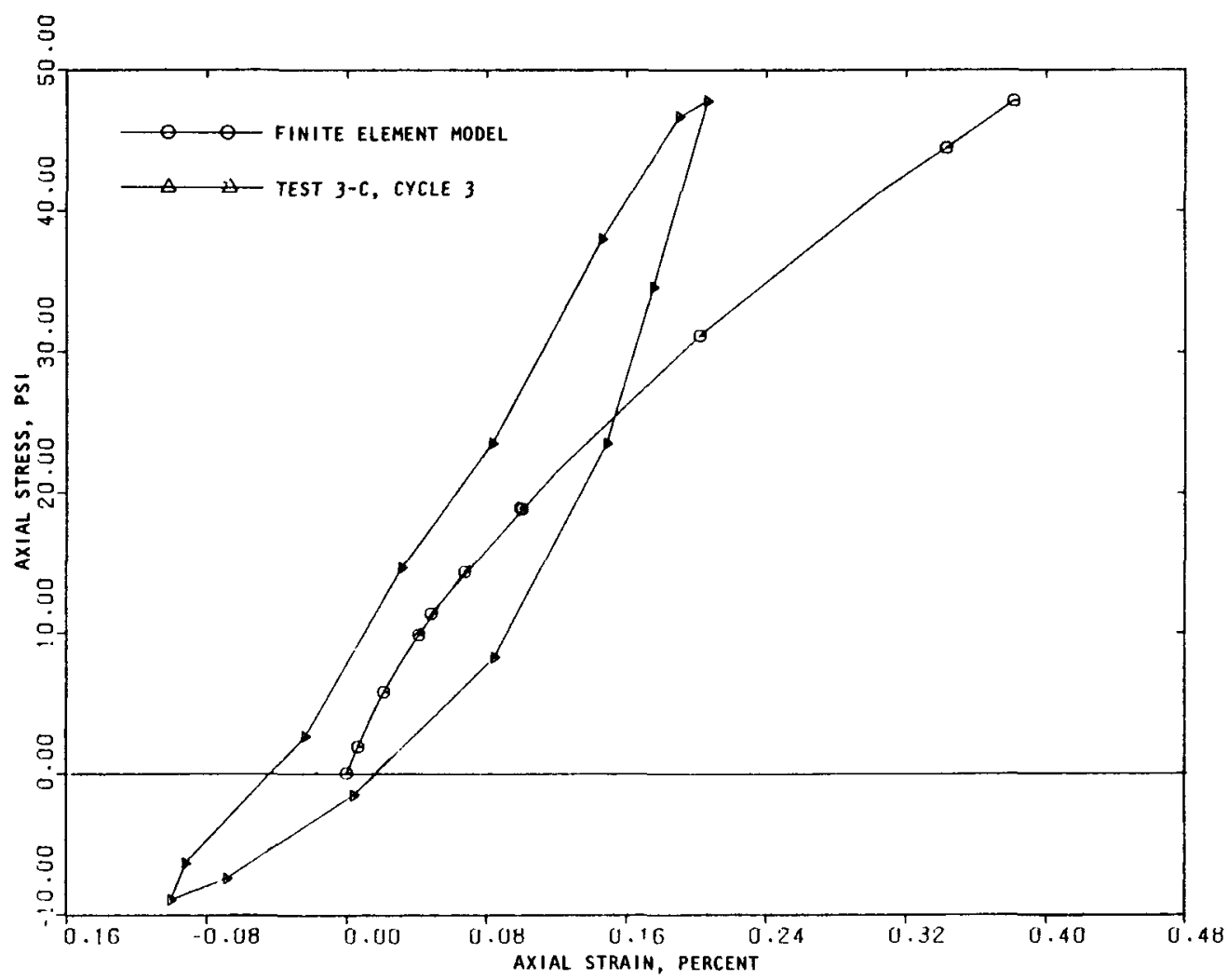

(b) Comparison with Test 3-C

FIGURE A-2. COMPARISON OF FINITE ELEMENT MODEL WITH TRIAXIAL TEST DATA AT 18 PSI CONFINING PRESSURE 
The finite element model and the triaxial data are not in agreement as to the magnitude of the strain at any point in the load cycle. The triaxial data indicates that permanent compaction of the soll sample has taken place during the first load cycle. In the case of Test $1-B^{\prime}$ (see Fig. A-la) the test sample has compacted to the extent that the origin of the stressstrain coordinate system no longer lies within the hysteresis loop. The finite element model has not been designed to take this effect into account. However, the permanent compaction phenomenon appears to be related to the behavior of sand under the influence of normal stresses. It would appear that there should be no compaction at all due to the application or removal of shear stresses. In view of the fact that the sand model is used in a configuration which approaches loading in pure shear, the omission of this effect from the sand model appears justified. Furthermore, the values of shear modulus obtalned from the finite element sand model are in reasonably good agreement with the state-of-the-art curve obtalned from SW-AJA, 1972. This fact adds greater support for the hypothesis that permanent compaction under normal states of stress need not be considered in the finite element model.

In Fig. A-lb, the behavior of the finite element model is compared with Test 1-C'. As is the case in Fig. A-la, the slope of the stress/strain path appears to be in good agreement among the three curves shown. The area contained in the hysteresis loop of the sand model appears to be slightly low, but comparison of Cycles 2 and 4 of Test 1-C' shows that the area of the loop is not consistent even between different cycles of the same test. Again the tendency for permanent compaction is evident in the triaxial data, and continues to take place to a considerable extent even between Cycles 2 and 4 . 
Fig. A-2 shows the comparison between model behavior and triaxial data under a confining pressure of 18 psi. In that the finite element calculation has been run at a confining pressure of 6 psi, this comparison is of less interest than that of Fig. A-1. Nevertheless, excellent agreement between the model and Test $3-B$ is evident in Fig. A-2a. While Fig. A-2b shows the finite element model to be too soft at a stress level of 50 psi, it should be kept in mind that this stress level is much greater than any encountered in the finite element calculation.

\section{A-2 BEHAVIOR OF MODEL UNDER CYCLIC SHEAR LOADING}

The finite element sand model was subjected to cyclic loading in pure shear at several strain levels. Throughout these tests, the confining pressure was taken to be 6 psi. The results of these tests are shown in Fig. A-3. Experimental data does not accompany these curves in that the triaxial tests did not measure the shear strain directly.

lJote, particularly from Figs. A-3d and A-3e that for large shear strains, the second cycle of loading reaches a higher stress value than the first. It does so, however, while never allowing the second cycle to possess a tangent shear modulus which exceeds that of the first cycle at the same stress level. Thus the phenomenon shown in these figures is not work hardening. The higher stress obtained in the second cycle is due to the large amount of plastic flow which takes place during the unloading phase of the first cycle. Reloading during the second cycle, therefore, takes place over a much larger strain interval than in the original loading of the first cycle. This allows a higher stress to be attained, even though the modulus does not exceed that of the first cycle. 


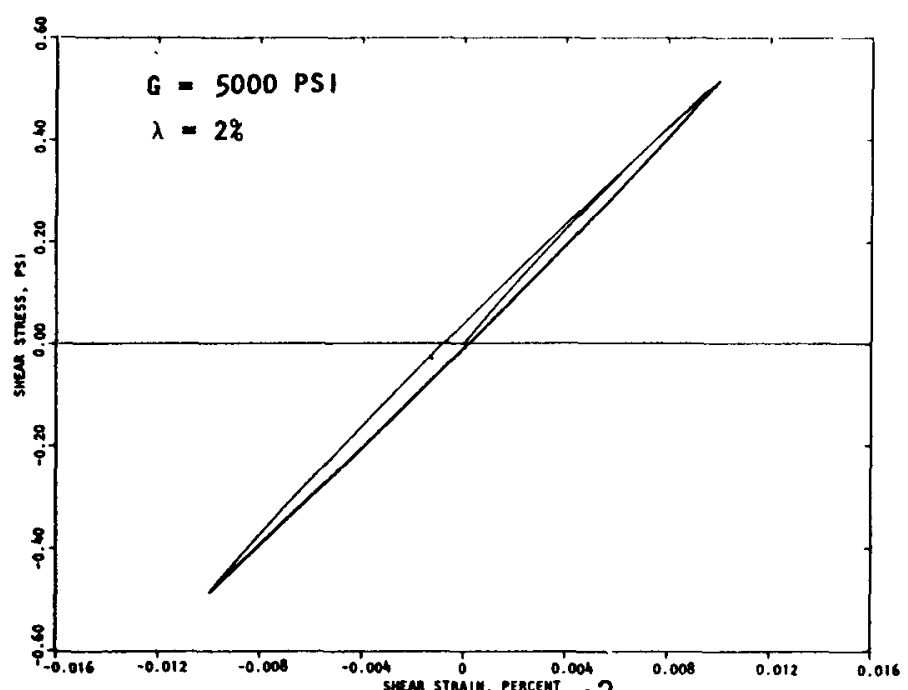

(a) Strain level $=10^{-2}$ percent.

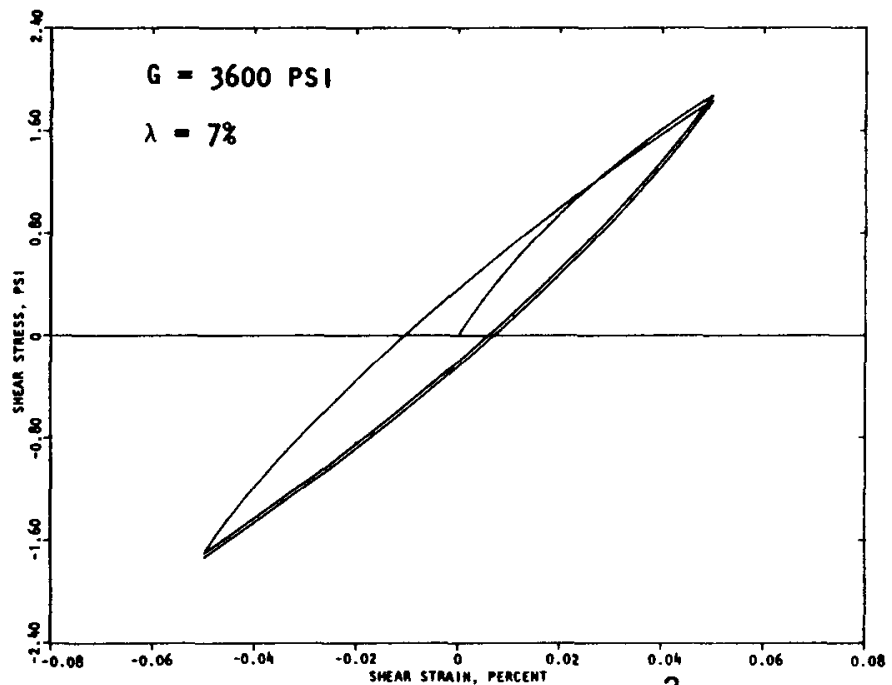

(b) Strain level $=5.0 \times 10^{-2}$ percent

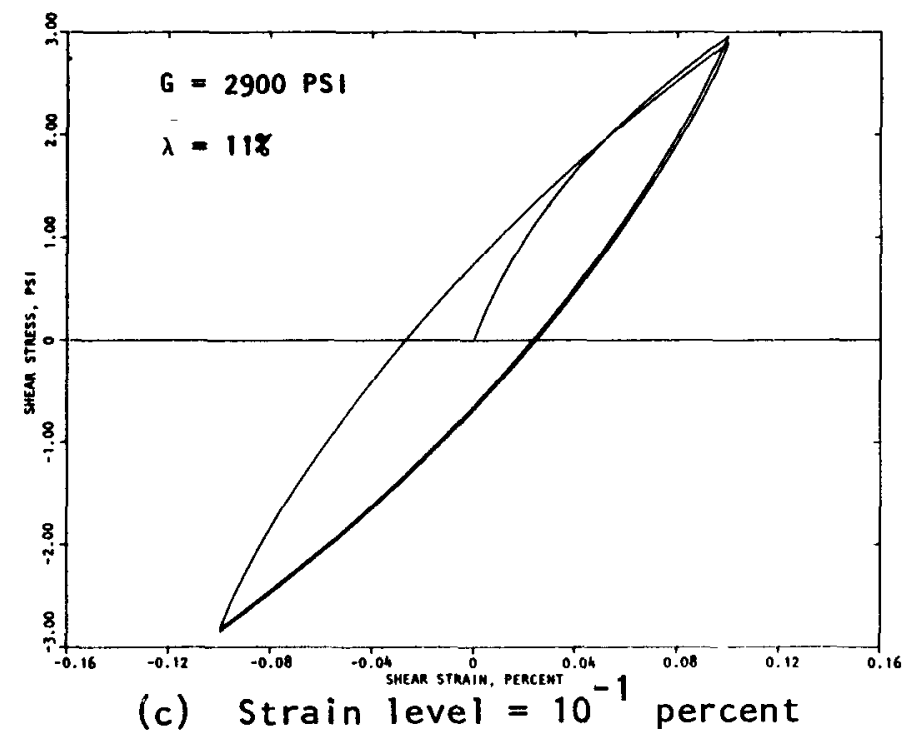

FIGURE A-3. CYCLIC SHEAR BEHAVIOR AT 6 PSI CONFINING PRESSURE 


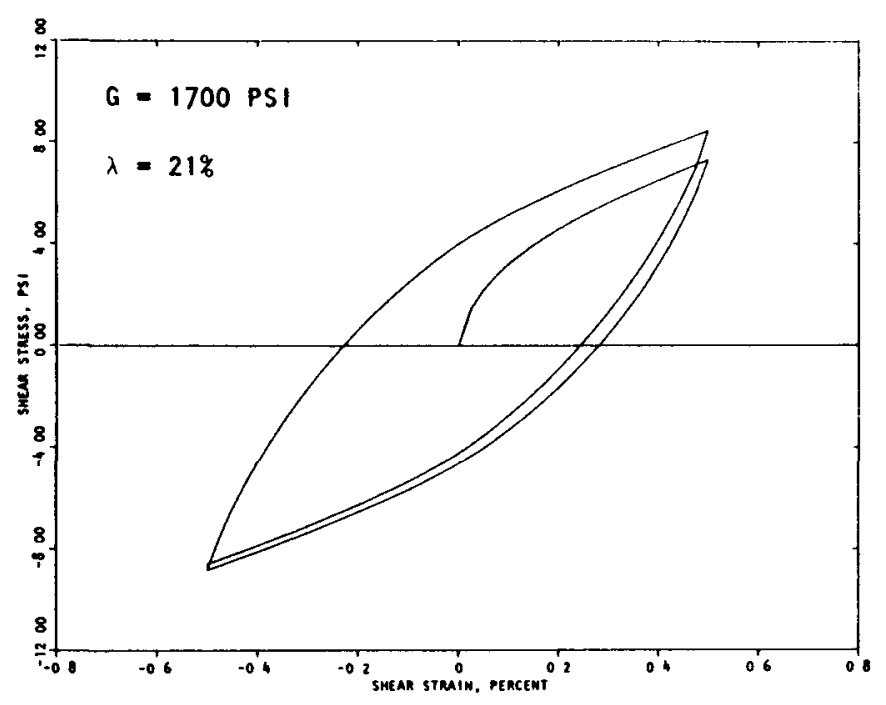

(d) Strain level $=5.0 \times 10^{-1}$ percent

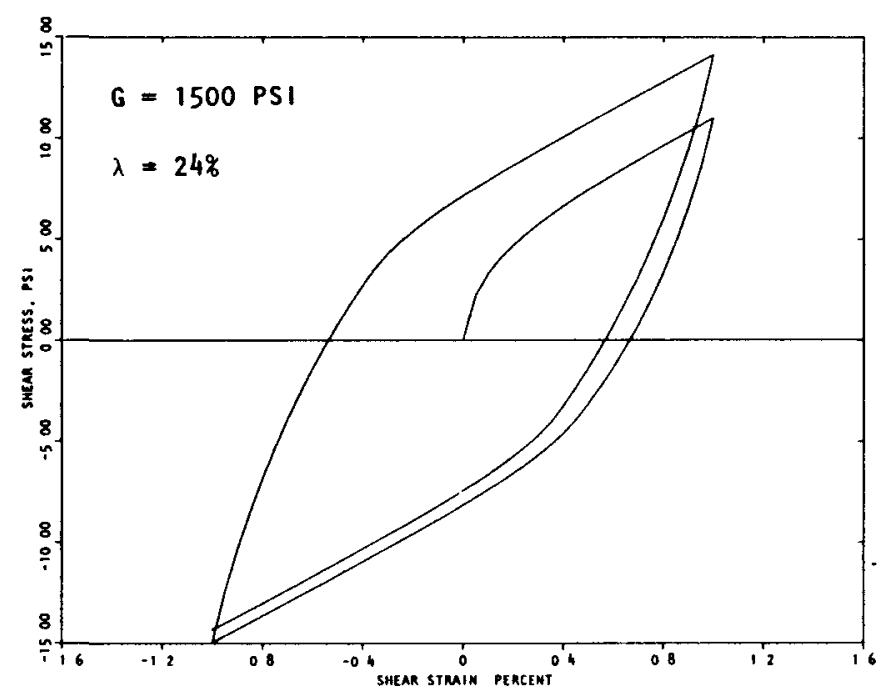

(e) Strain level $=1$ percent

FIGURE A-3. CYCLIC SHEAR BEHAVIOR AT 6 PSI CONFINING PRESSURE (CONTINUED) 
A secant shear modulus, G, and a damping ratio, $\lambda$, have been measured for each of the stress/strain loops of $\mathrm{Fig} \cdot \mathrm{A}-3$ according to the method outlined in Fig. A-4. These values are plotted in Fig. A-5 together with the state-of-the-art curves of SW-AA, 1972, and the interpretation of the triaxial data based on an assumed value of 0.3 for Poisson's ratio. The shear modulus comparison is presented in Fig. A-5a, and the damping ratios are compared in Fig. A-5b. The fact that the results using the finite element sand model, in most cases, fall within a range defined by the state-of-the-art curve and the constant Poisson's ratio interpretation of the triaxial data indicates that the values generated by the model are realistic at a confining pressure of $6 \mathrm{psi}$.

\section{REFERENCES}

SW-AJA (1972)

"Soil Behavior Under Earthquake Loading Conditions: State of the Art, Evaluation of Soil Characteristics for Seismic Response Analyses," A Joint Venture of Shannon \& Wilson, Inc., and Agbabian-Jacobsen Associates, Jan. 


\section{DEFINITIONS}

SHEAR MODULUS, $G=\frac{G_{2}}{1}$

DAMPING RATIO, $\lambda=\frac{A_{L}}{4 \pi A_{T}}$

$A_{L}=$ AREA OF LOOP

$A_{T}=$ AREA OF TRIANGLE $\backslash \backslash \square$

SHEAR STRESS

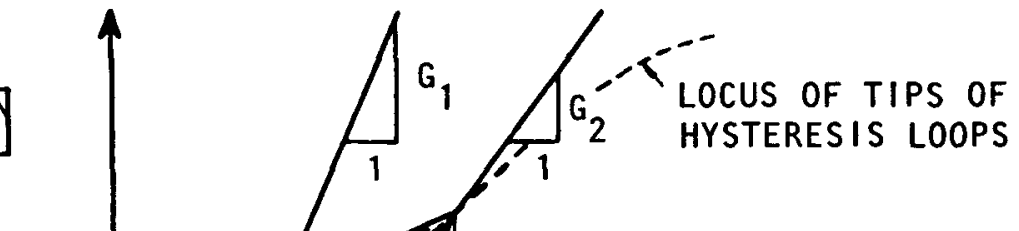

SHEAR STRAIN

AMPLITUDE $\rightarrow$

NOTE :

DEFINITION OF MODULUS, DAMPING

AND STRAIN AMPLITUDE NOTED ON

FIGURE APPLIES TO LARGER

HYSTERESIS LOOP ONLY.

FIGURE A- $\overline{4}$. HYSTERETIC STRESS-STRAIN RELATIONSHIPS AT DIFFERENT STRAIN AMPLITUDES 


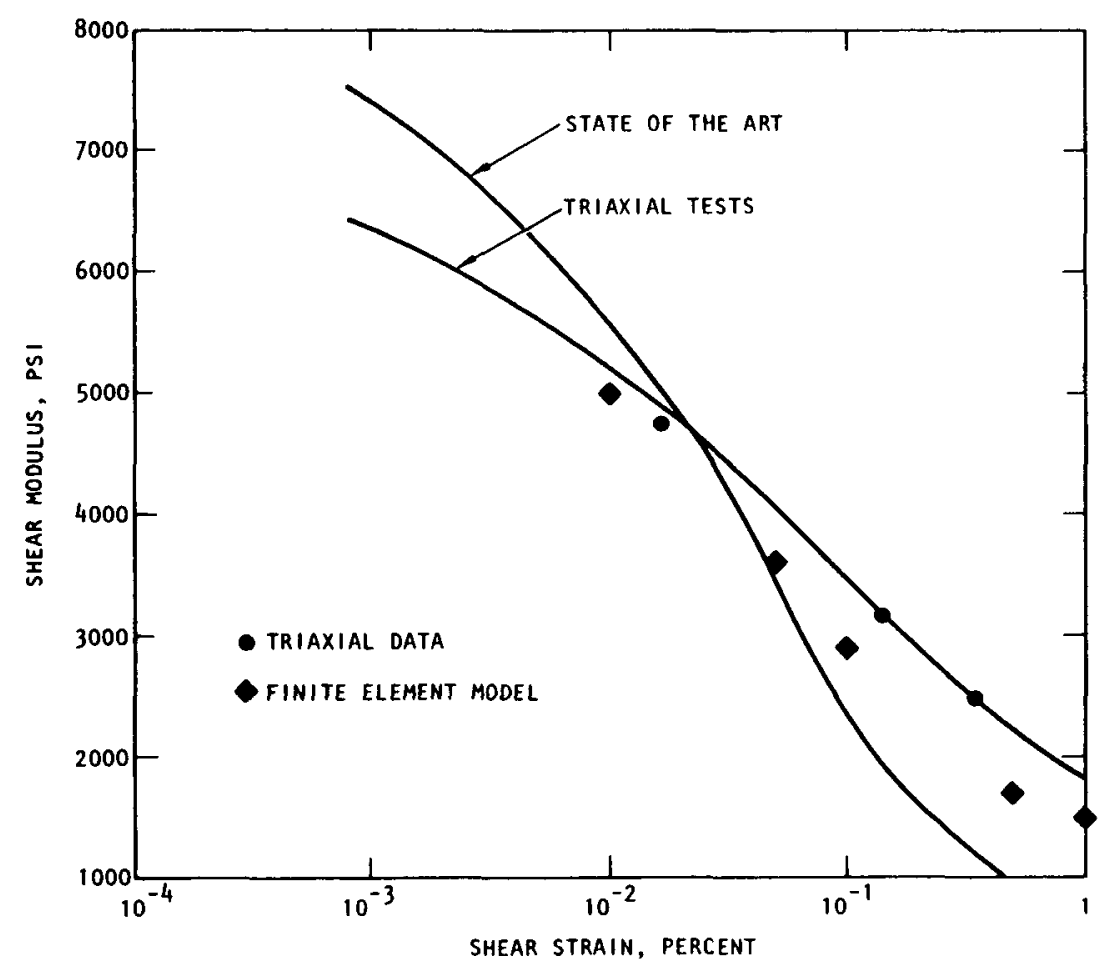

(a) Shear modulus

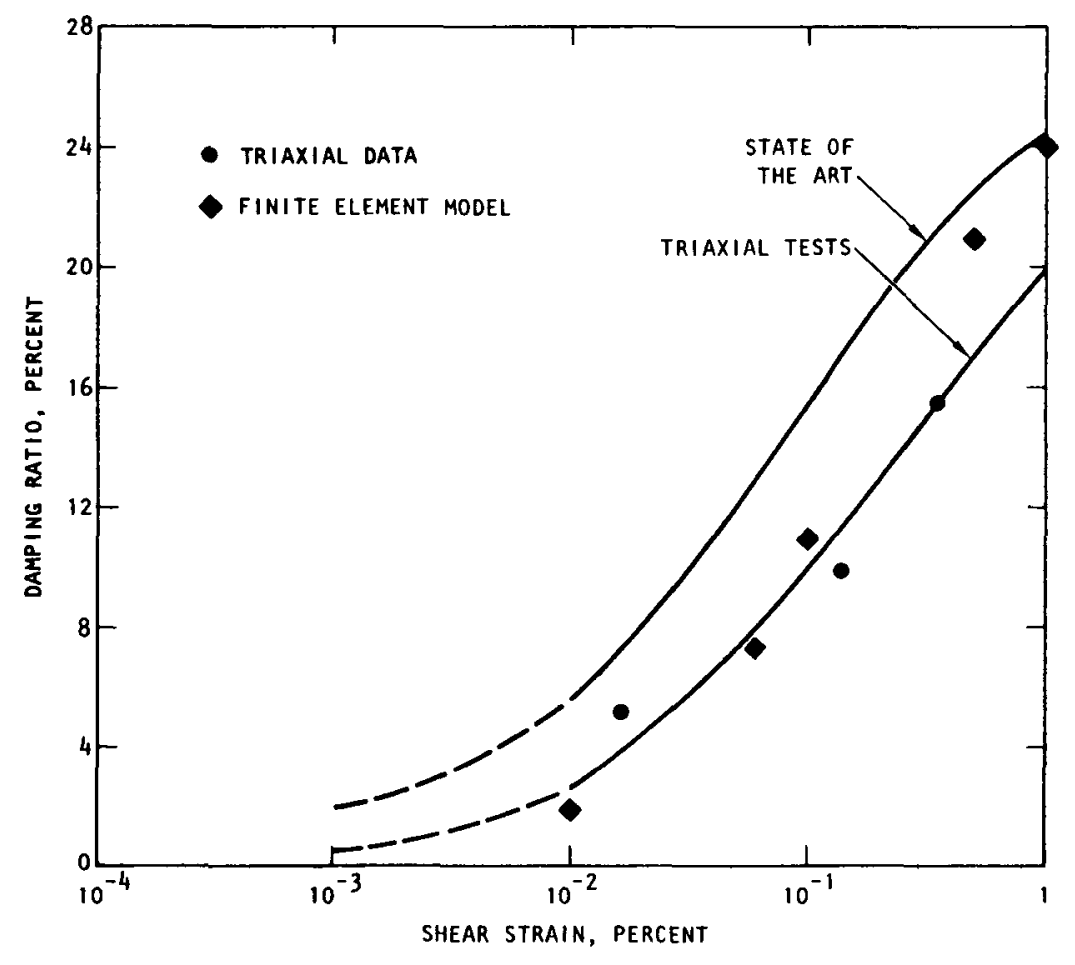

(b) Damping ratio

FIGURE A-5. COMPARISON OF FINITE ELEMENT SAND MODEL WITH TEST DATA AND STATE-OF-THE-ART DATA FOR 6 PSI CONFINING PRESSURE 
APPENDIX B

PNEUMATIC ANCHOR RESULTS 
As pointed out in Chapter 5, primary waves are dominate in the close range zone. These strong waves may be caused by unavoidable imperfections in the design of the complex hydraulic anchor assembly. The central hydraulic ram is of high mass and when excited is thought to (by its inertia) produce a large primary wave. To investigate this cause, a smaller diameter prototype light-weight central mass anchor designed for use at greater test depths was tested as a part of this program.

The new anchor shown in chapter 2 was made up of three rigid smooth cylindrical plates, cut from a 4-inch O.D., .636-inch thick steel pipe, which were pressed against the soil mass by an air inflated high pressure, reinforced pneumatic rubber packer. The prototype used in these tests was placed in the hole left by the hydraulic anchor after completion of this initial test phase. Fill was placed around the anchor to fill in the extra void space. Because the new anchor was smaller in diameter, about 4 to 5 inches in the fully retracted position, the spacing from the wall to each recording station was slightly lengthened. The vertical length of the anchor in contact with the soil was the same, 4 feet, the lateral surface was smaller by approximately 50 percent, and the total weight was smaller by 30 percent.

The following paragraphs present the results of a series of tests performed with this pneumatic anchor.

\section{B-1 CHARACTERISTICS OF THE PULSE}

The velocity-time histories obtained in six successive tests, with the pneumatic anchor, showed that the general shape and amplitude characteristics of the pulses follow the same trends of those observed with the hydraulic anchor. 
Comparisons of the ratios of main to primary components of particle velocities and duration times in general verify that the relative importance of the primary pulse is indeed smaller for the pneumatic anchor than the hydraulic system. In fact, the primary particle velocity amplitude is practically negligible beyond Sensor No. 5 at a radial distance of 20 inches from the center of the anchor. For a typical case of a 58 pound hammer falling six inches, the primary particle velocity (P-wave) decreases from 2.64 (in/sec) at station 1 to .01 (in/sec) in station 13, while the main particle velocity (s-wave) decreases from 3.38 (in/sec) in station 1 to .13 (in/sec) in station 17 . The ratio of the main to the primary particle velocities increases from 1.28 at station 1 to 25 in station 5, while the ratio of the corresponding duration times increases from 1.24 at station 1 to 8.23 at station 7. The time lag between primary and main pulses increases from .97 (msec) at station 1 to 5.7 (msec) at station 11 .

This indicates that the hydraulic anchor system, while producing a controlled wave rich in shear characteristics, is also generating p-waves. The smaller anchor, tested because it possibly represented a more ideal pure vertical shear impact in general, demonstrated just that. Two major differences in the two systems which probably account for this difference are l) the light central mass and 2) uniform vertical loading on the top of the plates. The smaller weight of the internal packer units is negligible when compared with the weight of the surrounding plates. Because the energy is applied to the plates directly internal movements and resisting inertia forces of the central packer are likely to be small, minimizing the possibility of transferring these internal forces laterally to the surrounding soil as P-waves. 
Since the diameter of the pneumatic anchor is smaller than the hydraulic anchor, the striker plate is likewise smaller and thus the possibility of achieving large eccentric loading conditions is reduced. An eccentric loading on a plate seyment can produce bending and in effect induce lateral compression force into the surrounding soil. This compressional stress wave will occur as p-waves. As a result of this study, weages were introduced on top of each plate segment on both anchor assemblies directly beneath the striker plate. The wedges produced point loads between the striking plate and the centroid of each plate segment. Subsequent production testing has demonstrated that the p-waves were minimized significantly by this more uniform loading condition.

\section{B-2 WAVE VELOCITY COMPUTATIONS}

The shear wave velocities were computed for the same series of tests above by different methods, including both the waveforms and various time history methods. The waveforms were computed from the displacement pulses at time intervals of.1 msec. The complete set of waveforms for these tests is plotted in Fig. B-l. From these curves, plus additional intermediate ones, a new set was formed to include only those waveforms whose crests were located just at a given station. This set is shown on Fig. B-2. The corresponding velocities are tabulated in Table B-1 along with those velocities determined by the other various time history methods.

The nomenclature for the wave velocities in this figure are as follows:

$$
\begin{aligned}
& \mathrm{V}_{1}=\text { wave velocity for peak } \dot{\mathrm{w}}_{1}, t_{1} \\
& \mathrm{~V}^{\prime \prime}=\text { same for second crossing } t^{\prime \prime}
\end{aligned}
$$




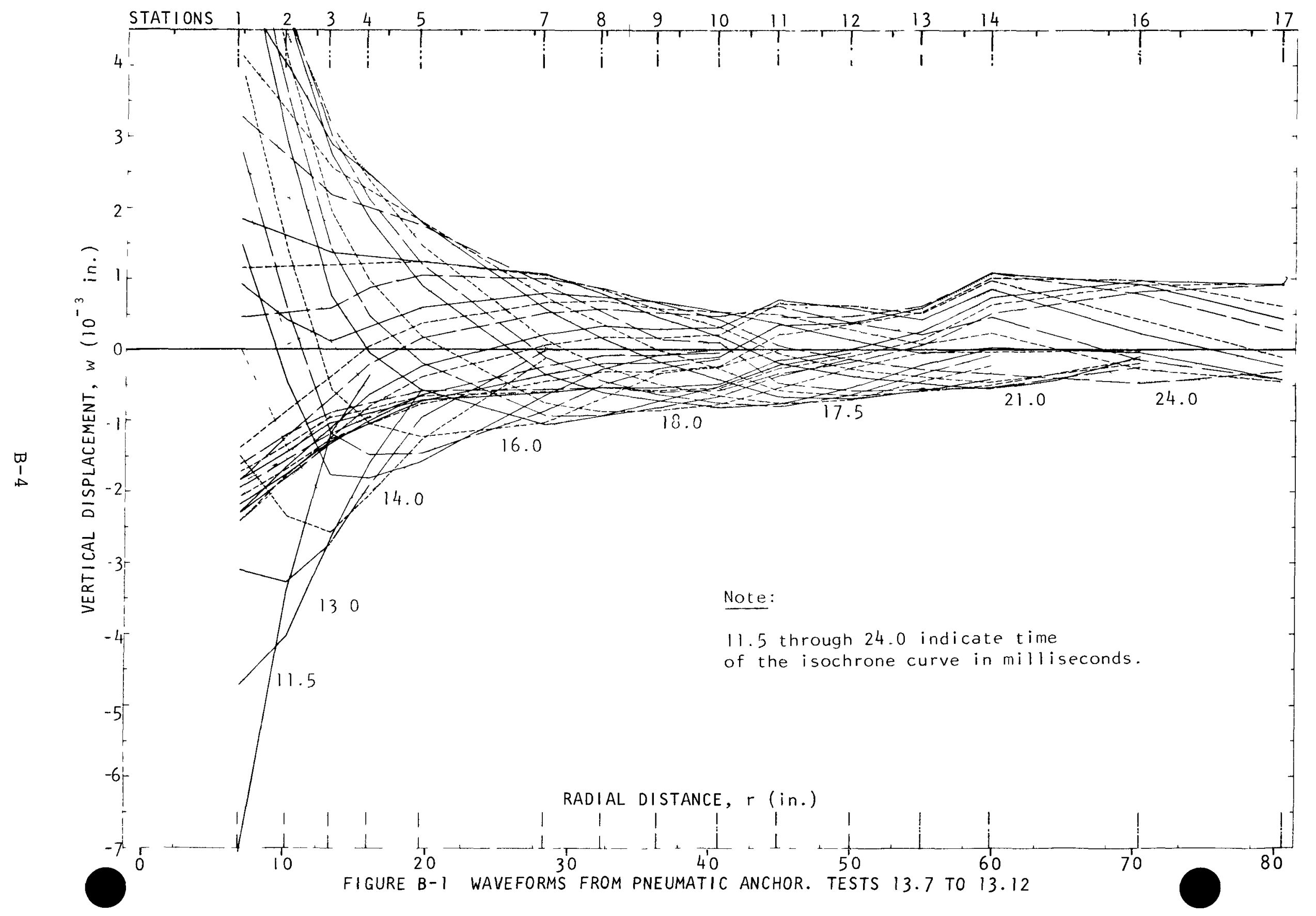




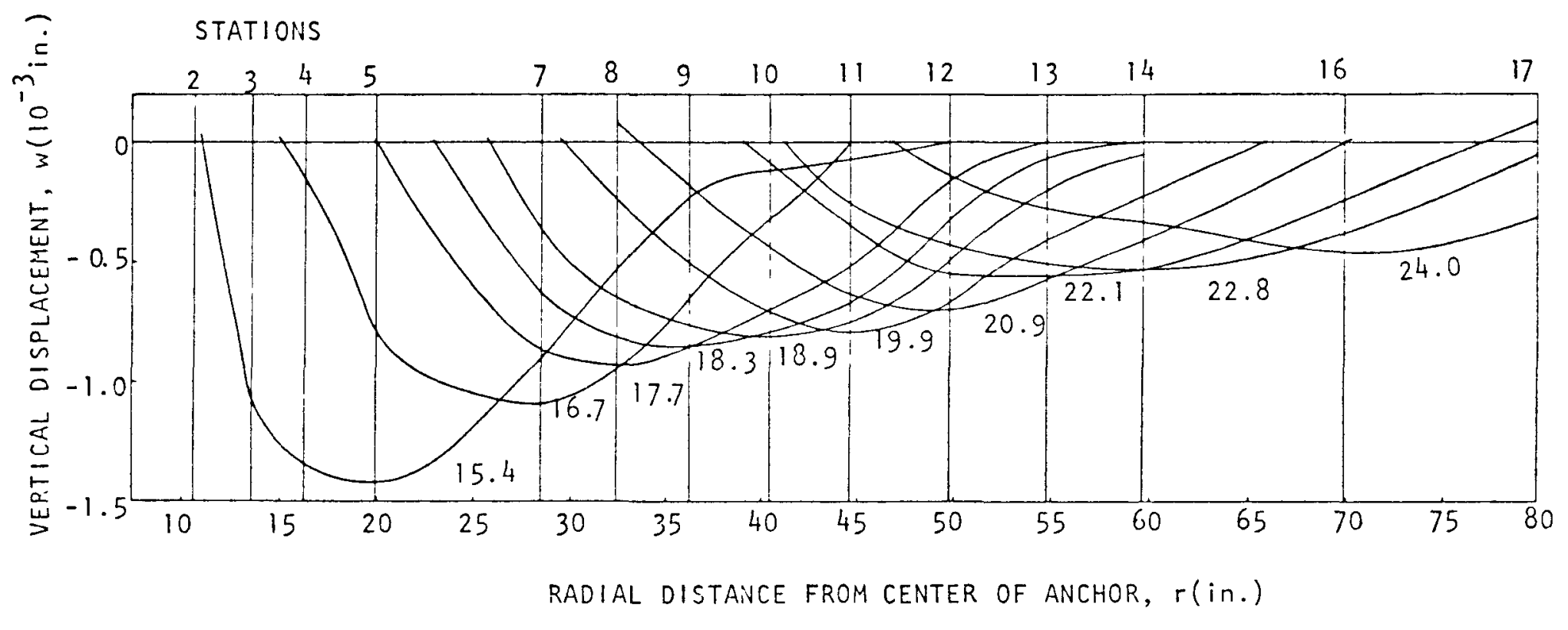

Notes:

1. 15.4 through 24.0 indicate time of the isochrone curve in milliseconds.

2. Waveforms selected for crest just passing through a receiving station.

FIGURE B-2 WAVEFORMS FOR PNEUMATIC ANCHOR. TESTS $13.7 \mathrm{TO} 13.12, \mathrm{~W}=58 \mathrm{lb} \times .5 \mathrm{ft}, \sigma_{\mathrm{j}}=140 \mathrm{ps} \mathrm{i}$ 


\section{Table $B-1$}

\section{Wave Velocity Computed from Different Methods}

Pneumatic Anchor

Tests $13.7-13.12, W=58 \mathrm{x} .5 \mathrm{lb} / \mathrm{ft}$,

Wave Velocity (fps) and Average Wave Velocity (fps)

\begin{tabular}{|c|c|c|c|c|c|c|}
\hline $\begin{array}{l}\text { Station } \\
\text { Number }\end{array}$ & $\begin{array}{l}\Delta r_{t} \\
(\mathrm{ft})\end{array}$ & $\begin{array}{c}\mathrm{V}_{1} \\
\text { (tps) }\end{array}$ & $\begin{array}{l}V^{\prime \prime} \\
\text { (fps) } \\
\end{array}$ & $\begin{array}{c}V_{2} \\
\text { (fps) } \\
\end{array}$ & $\begin{array}{c}\mathrm{V}_{\mathrm{C}} \\
\text { (fps) } \\
\end{array}$ & \\
\hline $1-2$ & .266 & 329 & 324 & 302 & & \\
\hline $2-3$ & .25 & 431380 & 281302 & $510 \quad 406$ & & \\
\hline $3-4$ & .218 & 303 & 307 & 234 & & \\
\hline $4-5$ & .302 & $254 \quad 280$ & $340 \quad 324$ & $408 \quad 321$ & & \\
\hline $5-7$ & .715 & 533 & 441 & $(325)$ & 550 & \\
\hline $7-8$ & .328 & 746 & 444 & $(439)$ & 328 & \\
\hline $8-9$ & .322 & 596 & 430 & 460 & 537 & \\
\hline $9-10$ & .354 & $466 \quad 587$ & $485 \quad 450$ & $506 \quad 432$ & 590 & 501 \\
\hline $10-11$ & .344 & 530 & 425 & 430 & 344 & \\
\hline $11-12$ & .426 & 609 & 474 & 560 & 426 & \\
\hline $12-13$ & .416 & $555 \quad 565$ & $467 \quad 455$ & $520 \quad 503$ & 397 & 389 \\
\hline $13-14$ & .406 & $(406)$ & 384 & $(666)$ & 581 & \\
\hline $14-16$ & .864 & 540 & 546 & 579 & 720 & \\
\hline $16-17$ & .825 & $(458) \quad 468$ & $594 \quad 508$ & $546 \quad 598$ & $(611)$ & 637 \\
\hline
\end{tabular}




$$
\begin{aligned}
& \mathrm{v}_{2}=\text { same for peak } \dot{\mathrm{w}}_{2}, \mathrm{t}_{2} \\
& \mathrm{v}_{\mathrm{c}}=\text { velocity computed from the waveforms }
\end{aligned}
$$

The results show that for the precision of the measurements, including the spacing of the sensors, and the resolution of the time of peaks and crossings, all methods give velocity results that, when averaged for groups 3 to 4 respective sensors in the free field, are in reasonable agreement. The maximum difference in velocity between the various methods was 45 percent.

\section{B-3 COMPARISON OF RESULTS FROM THE HYDRAULIC AND PNEUMATIC ANCHORS}

The tests with the pneumatic anchor were not intended to provide a rigorous quantitative correlation between both anchors; therefore, the conclusions are limited to general characteristics of the results.

1. The shear wave velocities given by both anchors in the free field are of the same order of magnitude and for both systems either method of computation of the wave velocity has been shown to give similar results with a general trend of increase of velocity with distance from the anchor.

2. There is a considerable difference in the close range zone. The pneumatic anchor produces a purer shear input and thus generates a weaker primary wave, permitting reduction of the distance of the close range to approximately station 4,16 inches from the center of the anchor.

These observations indicate that the nature or purity of the waves is dependent on the physical characteristics of the generating source. In the free field, however, where the main pulses are clearly defined, the properties of the soils are similarly well measured using either anchor system. 
APPENDIX C

SOIL PROPERTIES 


\section{APPENDIX C}

\section{SOII PROPERTIES}

The sand encountered at the Ft. Lawton site and used as fill material is the Esperance sand member of the Vashon Drift, a comformable late Pleistocene sequence of interglacial and glacial sediments (Mullineaux et al, 1965). This sand member appears to be chiefly a preglacial fluval and lacustrine deposit, that was laid down about 15,000 years ago after the lake was mostly filled with deeper lying silts and clays. Following its deposition, ice overrode the locality, compacting this sand under the weight of the heavy overlying glaciers. This compaction accounts for the high density characteristics apparent in this sand member.

\section{C-1 INDEX PROPERTIES}

Two samples taken from depths of 3 to 4.5 feet and from 8 to 9.5 feet classify as brown, silty, fine to medium SAND and gray brown, silty fine to medium SAND, trace of fine gravel. Both samples have 16\% silt and for both the $50 \%$ effective diameter is $0.26 \mathrm{~mm}$. Both samples are very similar, except for an additional $10 \%$ content of fine gravel, which is found in the deeper sample. Because gravel was removed during the construction of the fill, the shallower sample is more representative of the materials tested by the impulse test. The grain size distribution for this sample is shown in Fig. C-l. Its natural water content is $11.8 \%$ and the specific gravity was measured at 2.59. The shape of the particles is subangular.

\section{C-2 MECHANICAL PROPERTIES}

The maximum dry unit weight of this sand fill determined by the Modified Proctor compaction test (ASTM D-1557-58T), gave an average value of $115 \mathrm{pcf}$. The minimum dry unit weight 


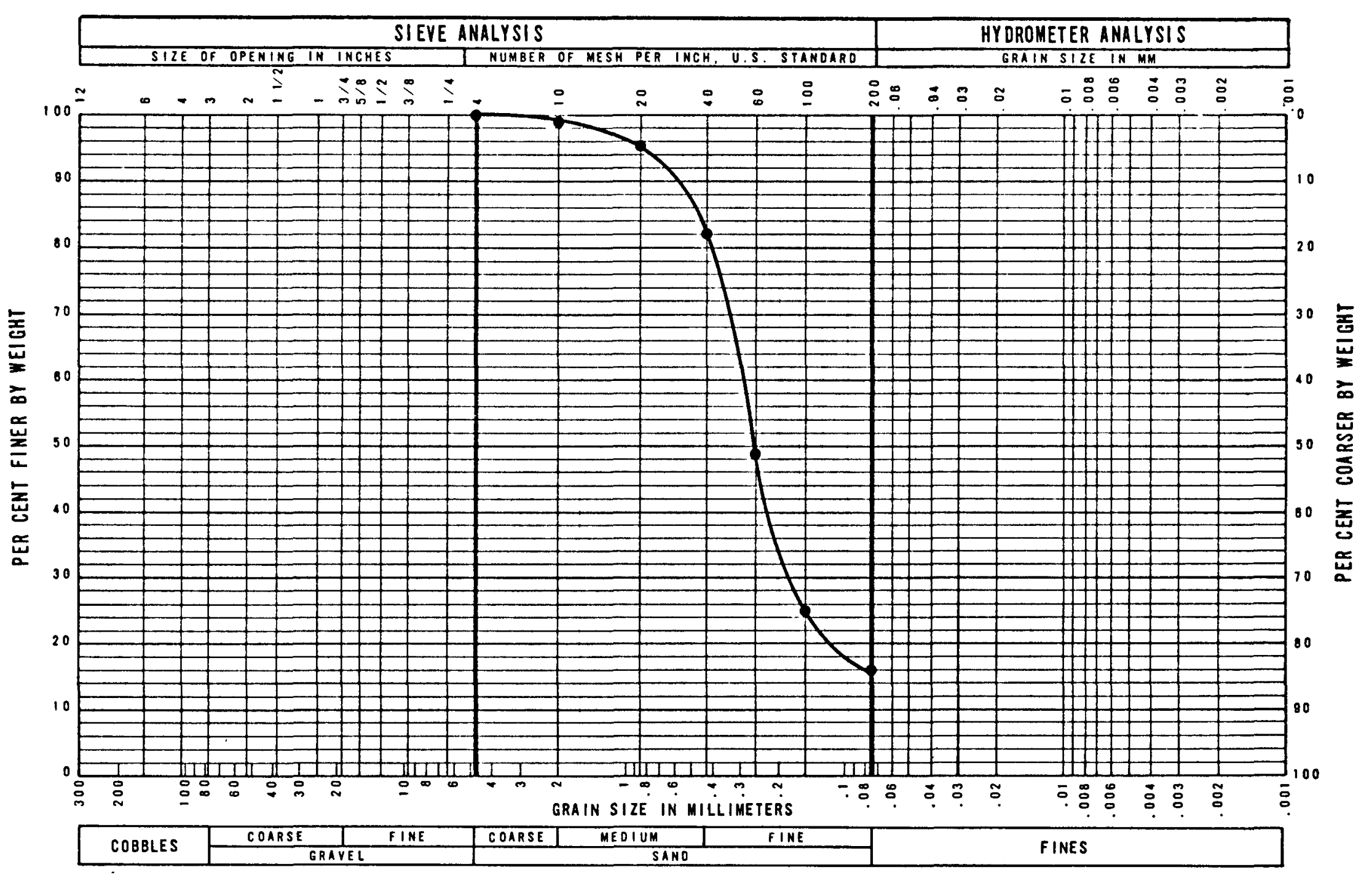

FIGURE C-1 GRAIN SIZE DISTRIBUTION. SOIL FOR ARTIFICIAL FILL TESTS. 
(ASTM D-2049-69) was 85.5 pcf. The average dry unit weight of the soil as compacted in the artificial fill was 99.3 pcf.

This corresponds to $86 \%$ of the maximum Modified Proctor unit weight. The maximum dry unit weight obtained by vibrations in the laboratory (ASTM D-2049-69) was 107.8 pcf.

The shear strength of the dry soil, compacted to 106.3 pcf, or 92\% of maximum Modified Proctor unit weight, was measured in a slow triaxial compression test, performed at an average rate of strain of .03\% per minute. The angle of internal friction for the range of confining pressures corresponding to an element at the mid-plane of the fill, and close to the anchor (confining pressures equal to .25 to .5 tsf) was 40 degrees, as shown in Fig. C-2.

The axial stress-strain curves for these triaxial tests give the following initial tangent modulus:

\section{Table $C-1$}

Initial Tangent Modulus from Triaxial Compression Tests

\begin{tabular}{|c|c|c|c|}
\hline Test No. & $3(\mathrm{psf})$ & $E \quad(p s f)$ & $\left(10^{-1} \div\right)$ \\
\hline 101 & 860 & $705,600-$ & $1.9-1.3$ \\
\hline 102 & 2,140 & $864,000-1,296,000$ & $2.5-.9$ \\
\hline 103 & 4,300 & $1,569,600-2,332,800$ & $3.0-1.0$ \\
\hline
\end{tabular}

\section{C-3 DYNAMIC PROPERTIES}

The dynamic properties of the compacted soils have been measured in the laboratory by means of cyclic triaxial and resonant column tests. The equipment for the cyclic triaxial tests is shown in Fig. C-3. The testing cell is made of stainless steel with a special stainless steel top cover equipped with a double-acting bellofram air cylinder connected to the 

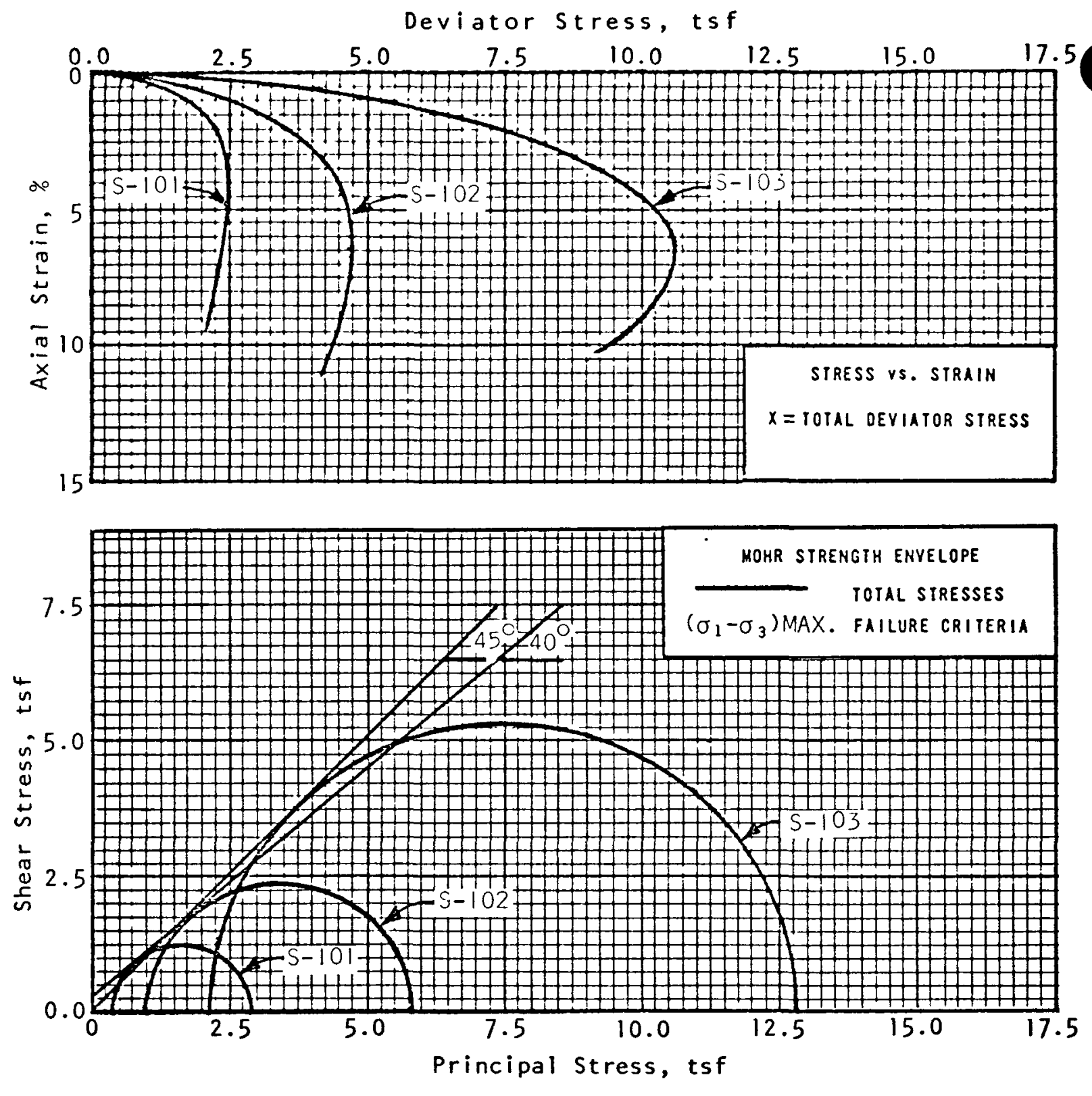

Test No.

$101 \quad 102 \quad 103$

Specimens Compacted

Eff. Confining

Pressure, tsf

$\begin{array}{lll}.43 & 1.07 \quad 2.15\end{array}$

Strain Controlled Test

Classification:

Gray, silty SAND

Ht. in.

$6.36 \quad 6.36 \quad 6.40$

Dia., in.

$\begin{array}{lll}3.00 & 3.00 & 3.00\end{array}$

Dry Unit Wt.pef $106.2 \quad 106.2 \quad 106.6$

* Tests performed on ovendried soil

Water Content, $\%$ *

Before Test

After Test

Avg. \% StrainlMin. $\quad .037 \quad 028 \quad .024$

FIGURE C-2 TRIAXIAL COMPRESSION TEST DRY, COMPACTED, SILTY SAND 


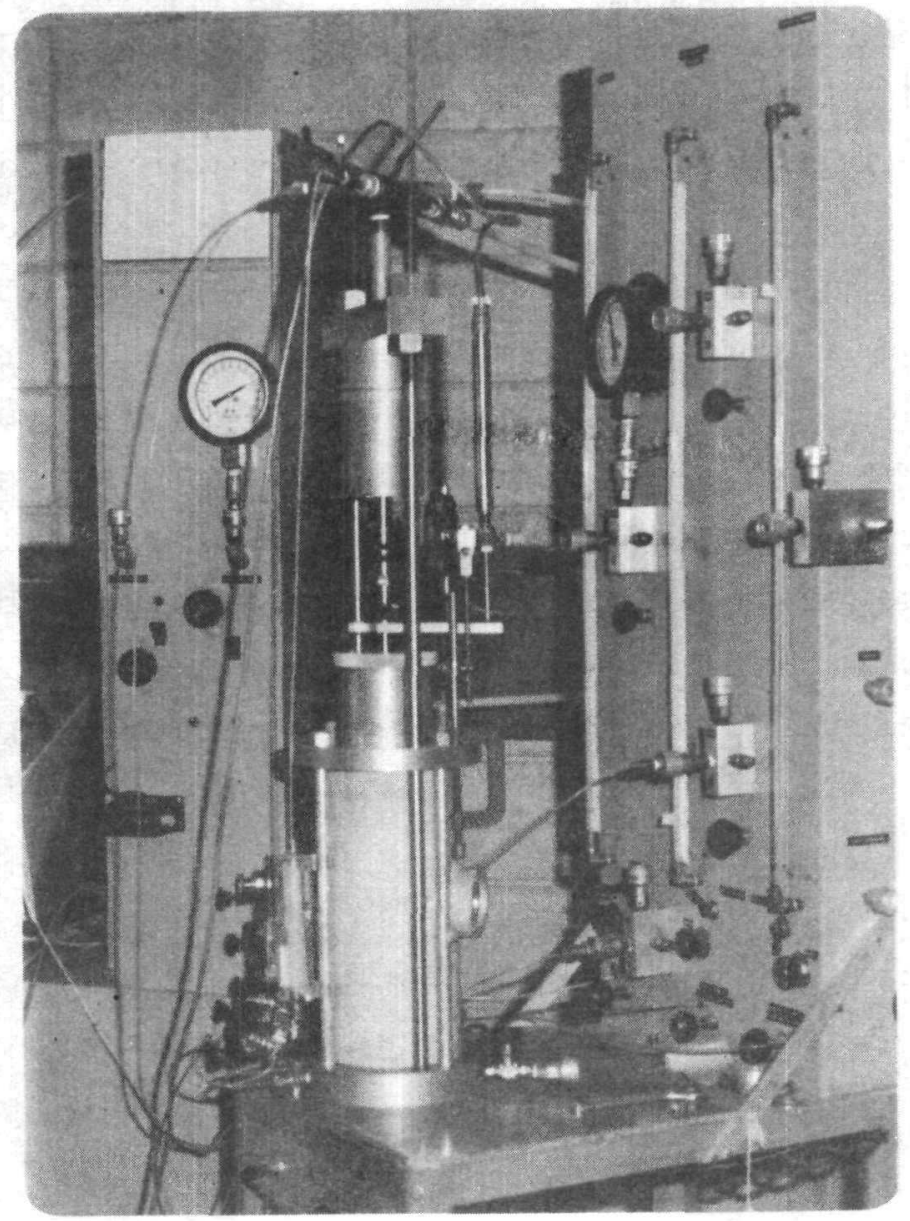

FIGURE C-3 LABORATORY EQUIPMENT FOR CYCLIC TRIAXIAL TEST

$$
\text { C }-5
$$


loading piston. The piston is fixed to the cap of the specimen to permit the application of upward and downward axial forces to the specimen.

The specimens are cyclically loaded as shown schematically in Fig. C-4. The reversal of shear stresses is obtained by maintaining the confining stress constant while adding and subtracting an axial increment of stress $\sigma_{d}$. In this manner, the stress conditions on a 45-degree plane are a normal stress $\sigma=\sigma_{3}=$ constant and a shear stress, which cyclically reverses between $\tau=+\frac{\sigma}{2}$ and $-\frac{\sigma d}{2}$. The normal stress is always kept positive. The reversal of shear stress is accomplished by means of an air operated single solenoid valve which causes the pressure acting on the bellofram to cycle between the equivalent maximum and minimum stress conditions. The result is an approximately sinusoidal stress-time history applied to the specimen under a maximum stress $\sigma_{d}$ at a frequency of 1 cycle per second.

The axial strains are measured with a linear variable differential transducer (LVDT) and the loads by a load cell mounted on top of the specimen. In addition, a pressure transducer is connected to the pressure supply line of the double acting bellofram to determine the magnitude of the pressure supplied. This extra measurement provides a check of the measurements of the load cell. The records of these measurements are registered simultaneously on two Sanborn 321 dual channel carrier amplifier-recorders.

The moduli and damping coefficients measured by the cyclic triaxial tests, for the confining pressure of 6 psi, have been summarized in the following table. 
a) CONSOLIDATION

DESIRED

CONDITION

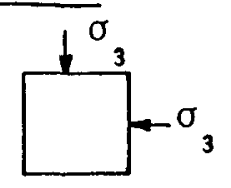

MOHR

DIAGRAM

$\tau$

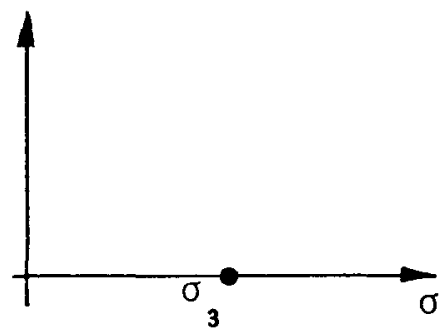

EQUIVALENT STRESS CONDITION

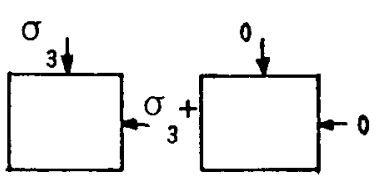

APPLIED STRESS IN

TRIAXIAL TEST

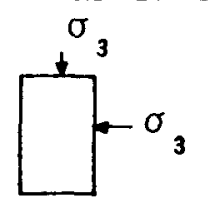

b) COMPRESSION AND SHEAR

c) TENSION AND SHEAR
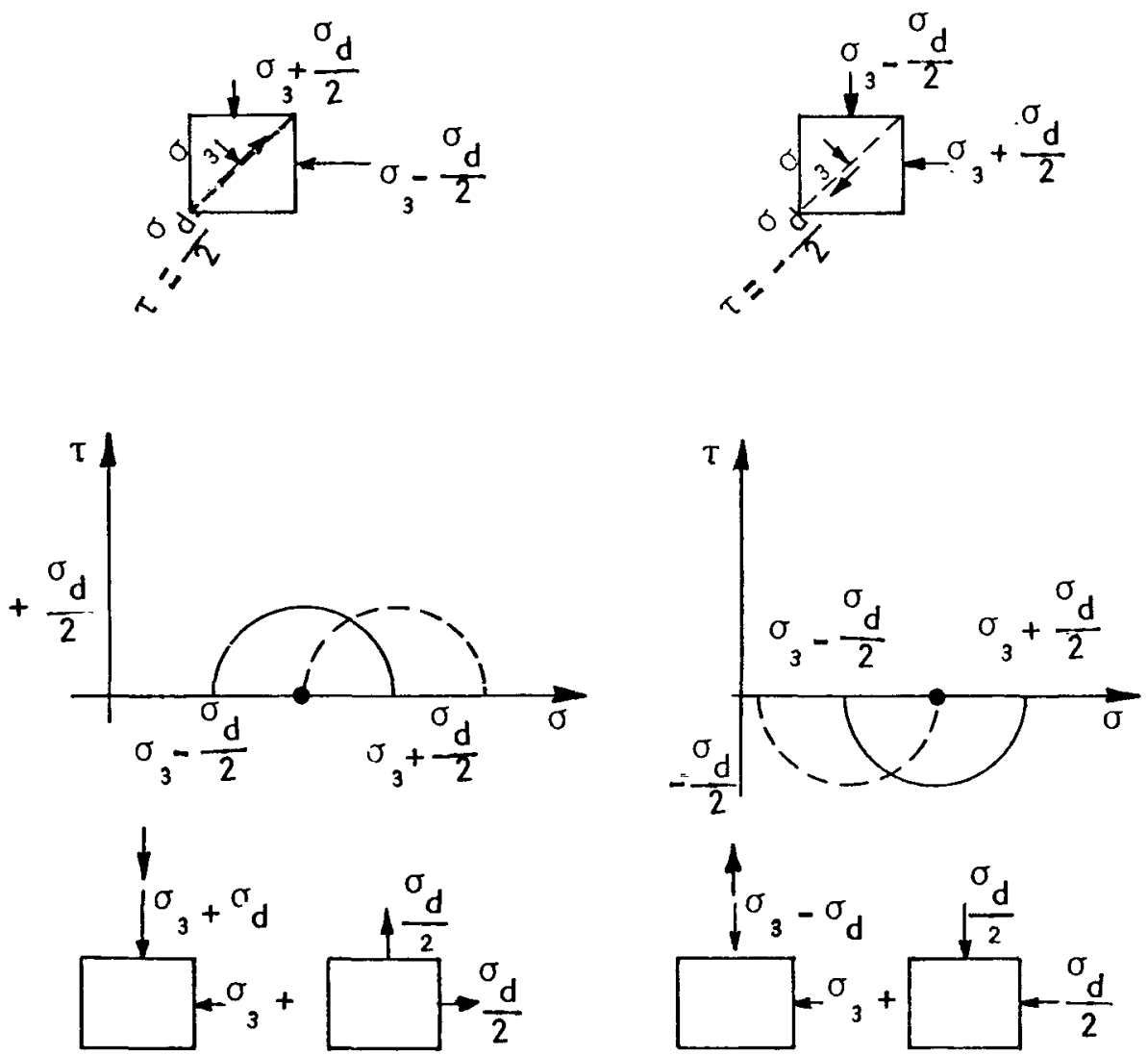

FIGURE C-4 CYCLIC TRIAXIAL TEST $-\bar{R}_{C Y}-$ LOADING CONDITIONS 


\section{Table $\mathrm{C}-2$}

Cyclic Triaxial Test Results:

Shear Modulus and Damping Versus Strain

Laboratory Results

for $\bar{\sigma}_{3}=6 \mathrm{psi}$
Computed Properties

for $v=0.3, \rho=3.41 \mathrm{~b} \cdot \sec ^{2} / \mathrm{ft} .4$

\begin{tabular}{|c|c|c|c|c|c|}
\hline $\begin{array}{l}\text { Axial } \\
\text { Strain } \\
\varepsilon \quad(\%)\end{array}$ & $\begin{array}{l}\text { Young's } \\
\text { Modulus } \\
\text { E (psi) }\end{array}$ & $\begin{array}{l}\text { Damping } \\
\lambda \quad(\%)\end{array}$ & $\begin{array}{l}\text { Shear } \\
\text { Strain } \\
\quad\left(\frac{\circ}{0}\right)\end{array}$ & $\begin{array}{l}\text { Shear } \\
\text { Modulus } \\
\text { G (psi) }\end{array}$ & $\begin{array}{l}\text { Shear Wave } \\
\text { Velocity } \\
V_{\text {s }} \text { (fps) }\end{array}$ \\
\hline .012 & 12,400 & 5.2 & .016 & 4,760 & 450 \\
\hline .106 & 8,300 & 9.9 & .14 & 3,190 & 368 \\
\hline \multirow[t]{2}{*}{.267} & 6,500 & 15.5 & .35 & 2,500 & 326 \\
\hline & & & $\star .001$ & 6,350 & 520 \\
\hline
\end{tabular}

The variation of shear wave velocity with shear strain for confining pressures of $6,12,18,24$ and $36 \mathrm{psi}$ is shown in Fig. C-5.

The resonant column tests were performed in the Combined Longitudinal and Torsional Modulus Device shown in Fig. C-6. The device subjects a cylindrical soil specimen to a sinusoidal vibration in a longitudinal or torsional mode such that the frequency corresponding to a state of resonance can be determined. For the specimen vibrating, as a fixed bottom, free top column, in the quarter-wave mode, the velocity of shear wave propagation is given by:

$$
\mathrm{v}_{\mathrm{s}}=4 \mathrm{f}_{\mathrm{t}} \mathrm{L}
$$

where $\mathrm{f}_{t}$ is the resonant torsional frequency in cycles per second, $L$ is the length of the specimen in feet and $V_{s}$ is the shear velocity in feet/second. 


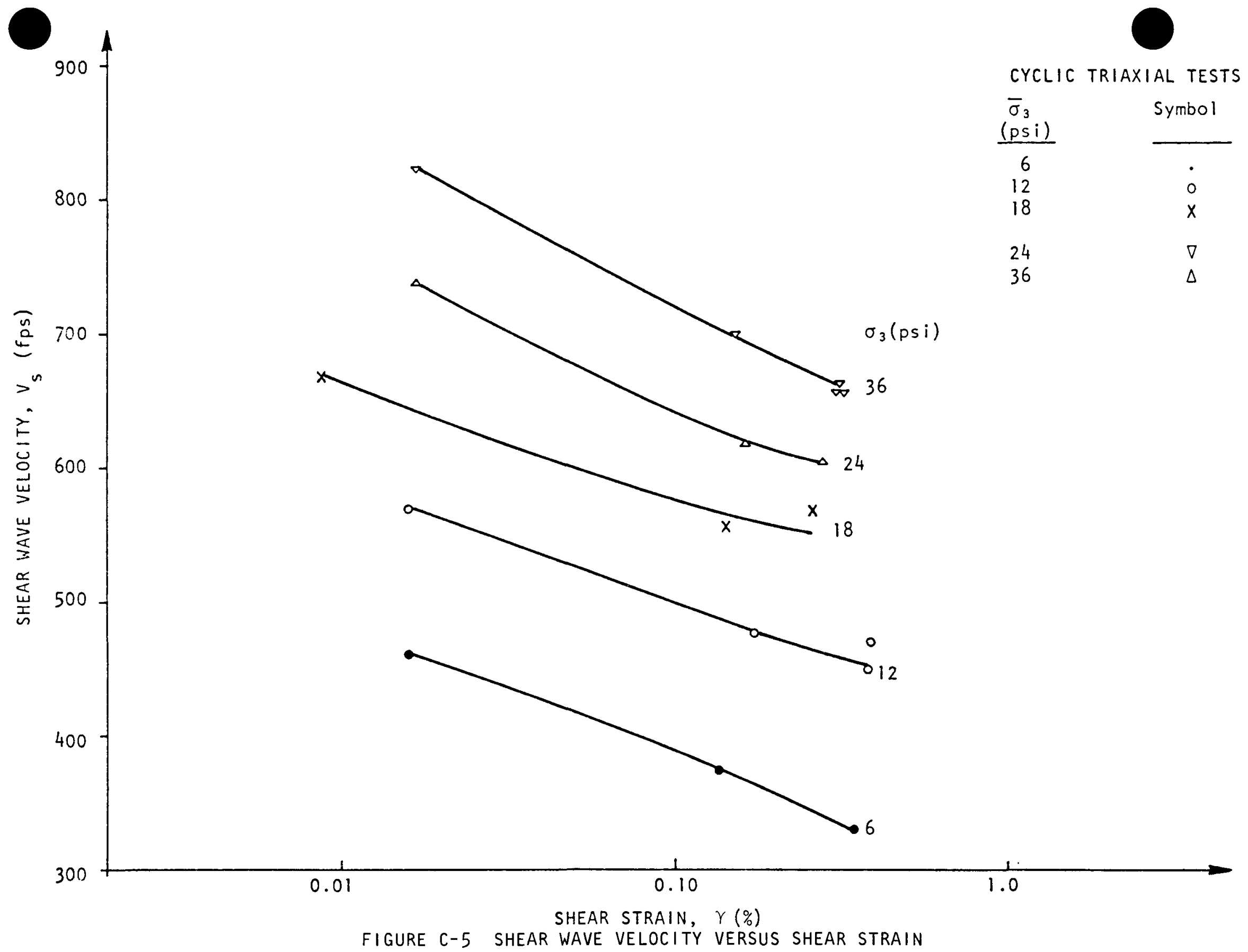




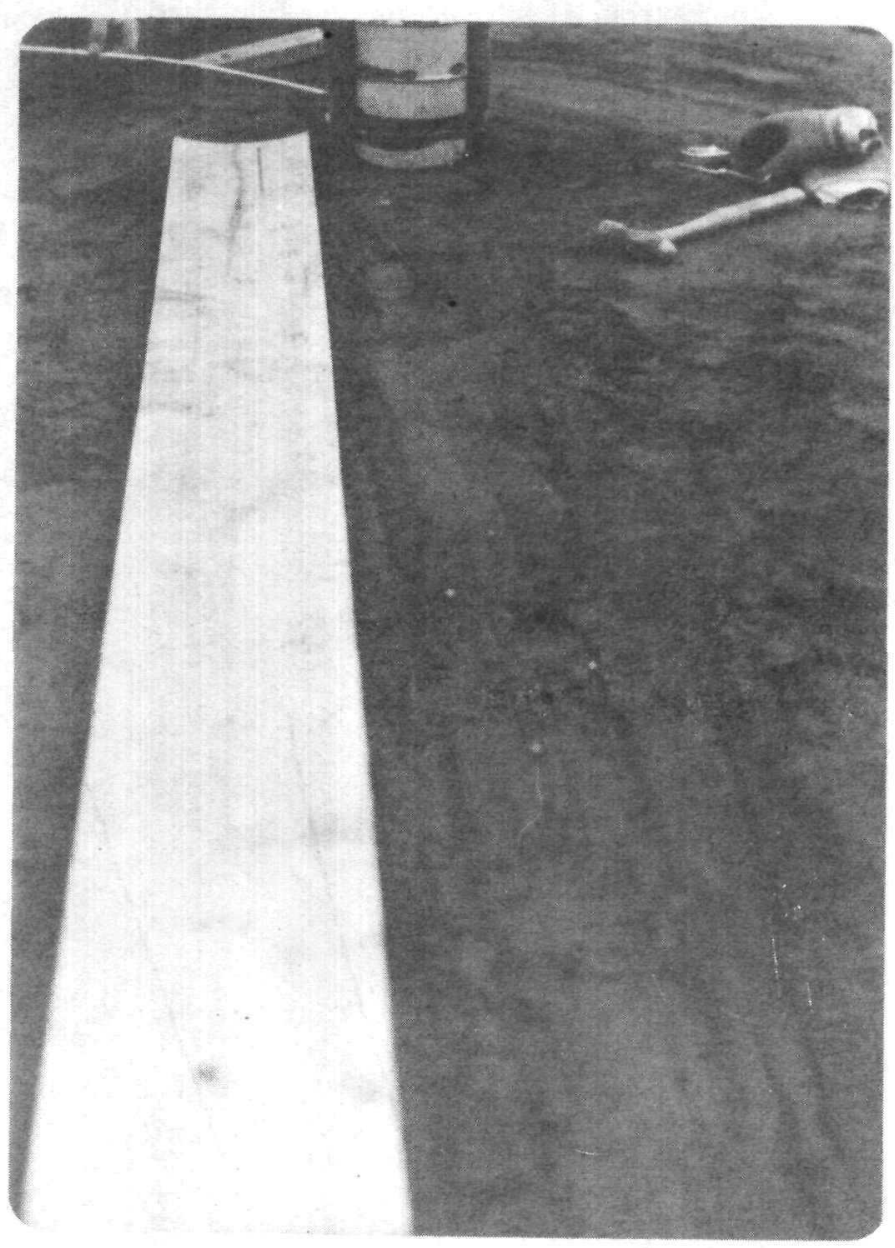

FIGURE C-6 RESONANT COLUMN

C -10 
Resonant column tests were performed at confining pressures of 10, 20, 40 and 80 psi, in a dry sample of soil compacted to 108 pcf. The results are shown in Fig. C-7 and summarized as follows :

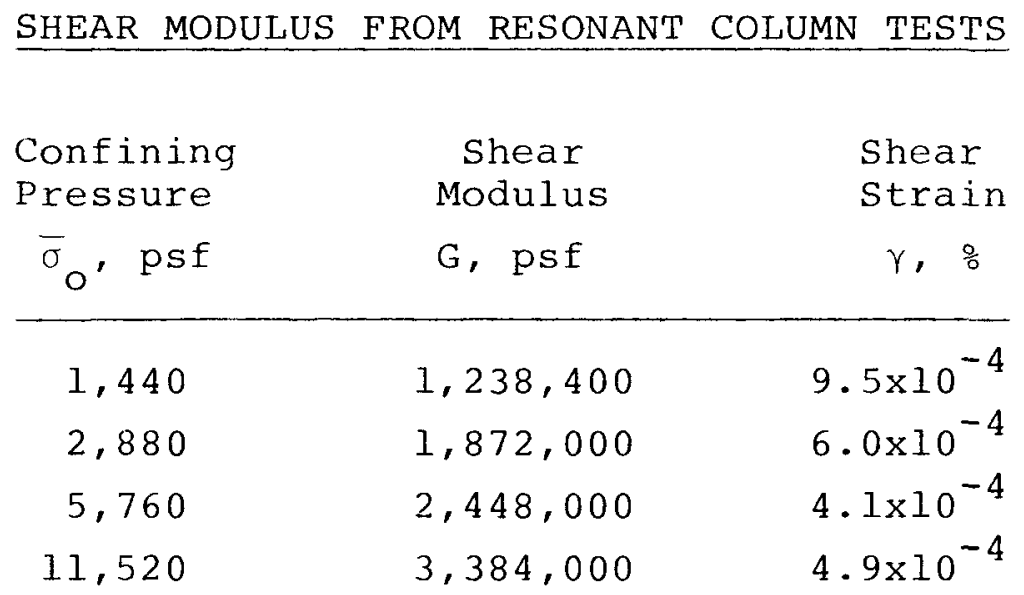

The results of all the measurements of shear modulus determined in the laboratory may be conveniently summarized in a plot of shear wave velocity versus shear strain, normalized for effective confining pressure and density. Fig. C-8 shows this plot in the form of the parameter $C$ versus $\gamma$, where $\gamma$ is the shear strain, in percent, and $C$ is the parameter from the equation:

$$
V_{S}=C \bar{\sigma}_{0}^{\frac{1}{4}} \rho^{-\frac{1}{2}}
$$

where: $V_{S}$ is the shear wave velocity in ( $\mathrm{ft} / \mathrm{sec}$ )

$\bar{\sigma}_{0}$ is the effective confining pressure in (lb/ft ${ }^{2}$ )

$\rho$ is the mass density in $\left(1 \mathrm{~b} \cdot \sec ^{2} / \mathrm{ft}{ }^{4}\right)$

and $C$ is a parameter in $\left(1 b^{\frac{1}{4}} / \mathrm{ft}^{\frac{1}{2}}\right)$ 


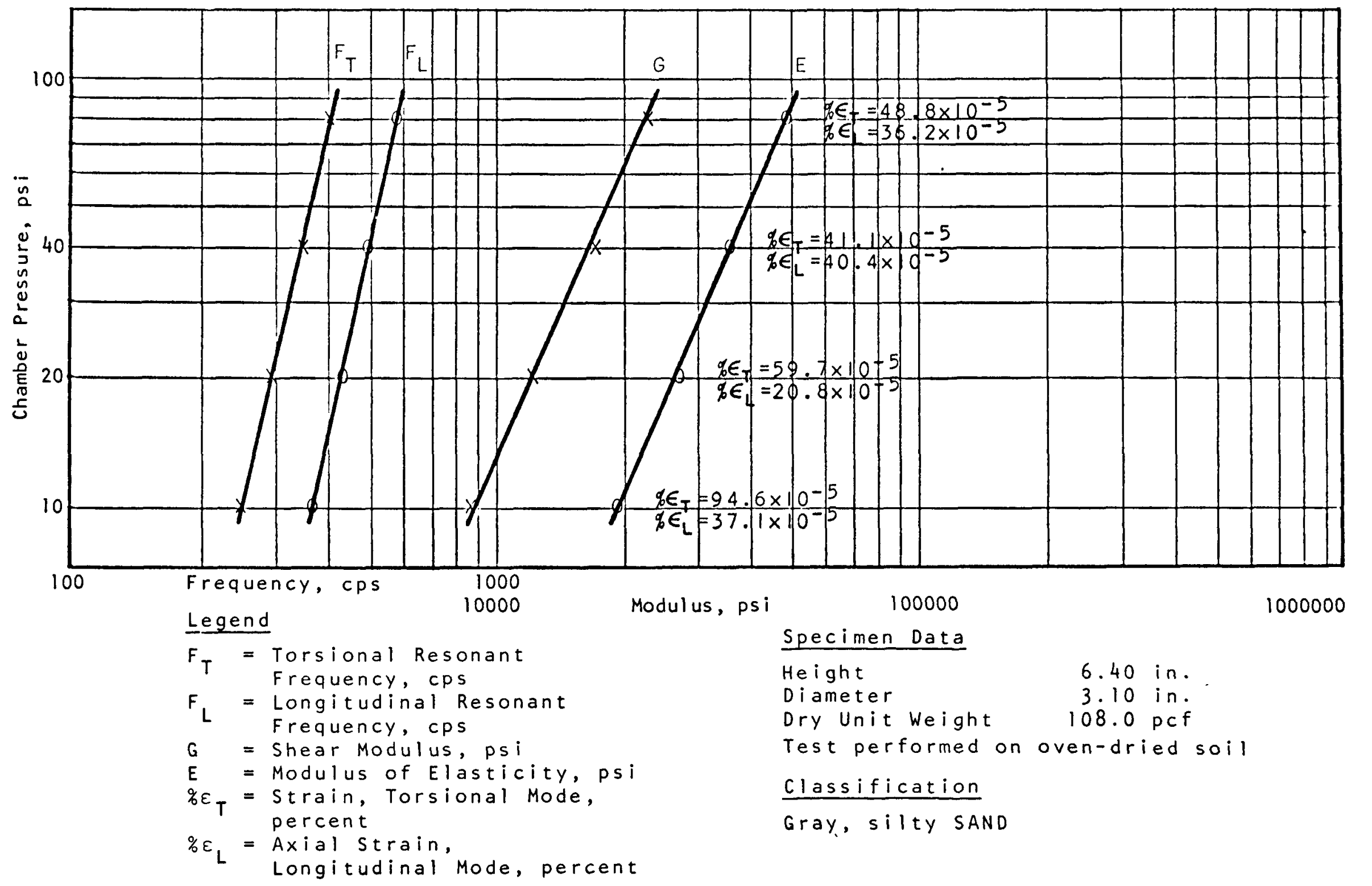

FIGURE C-7 RESONANT COLUMN TEST DRY, COMPACTED, SILTY SAND 


$$
v_{s}=\sigma^{\frac{1}{2}} \rho^{-\frac{1}{2}}
$$

Tests:

- Resonant Column

$x$ Cyclic Traixial

$\Delta$ Static Triaxial

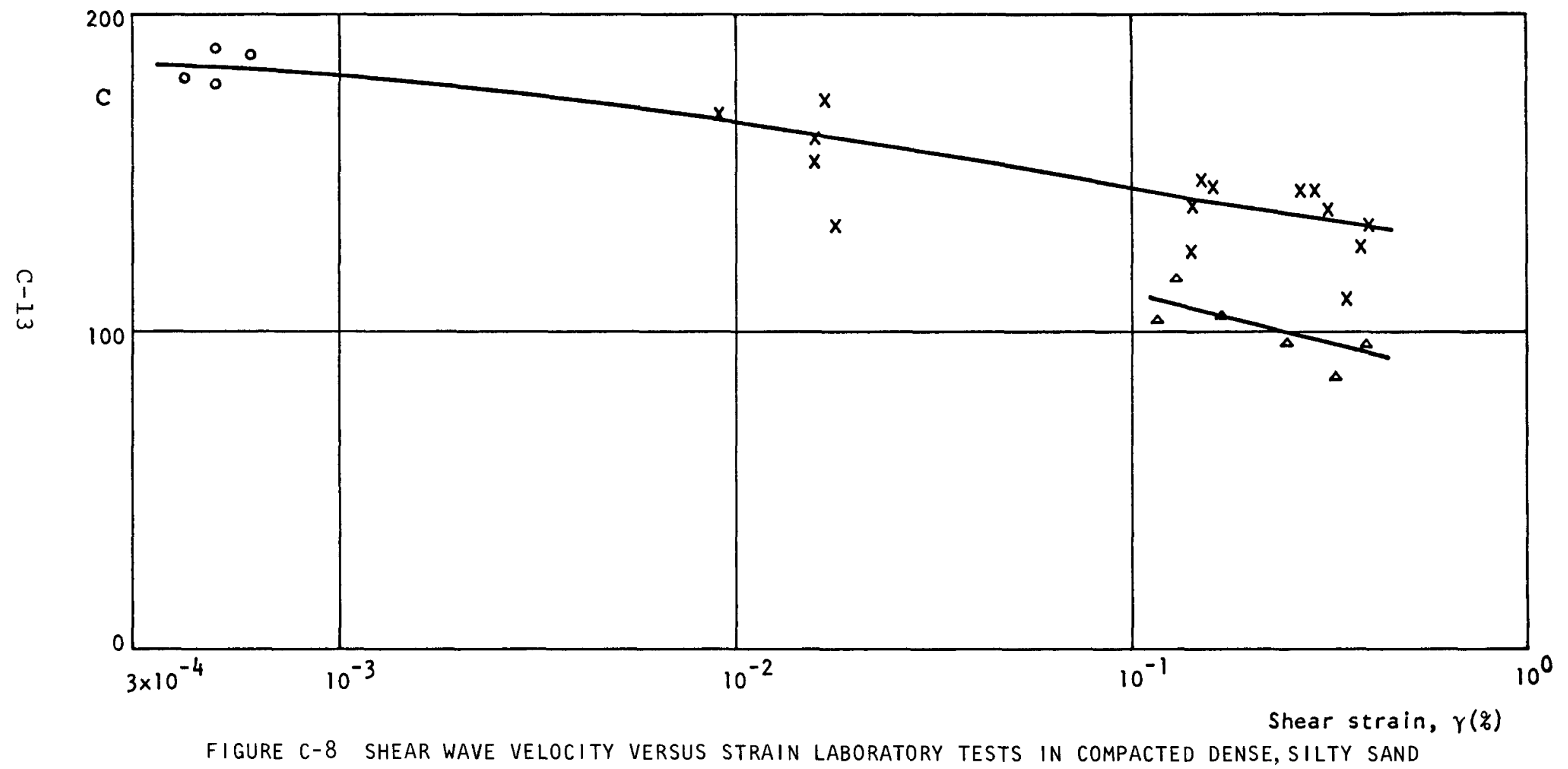


It is observed that for the same confining pressure and density, the shear wave velocity decreases by 10 percent when the shear strain increases from $10^{-3}$ to $10^{-2}$ percent, and by 14 percent when the strain increases from $10^{-2}$ to $10^{-1}$ percent. It is also observed that the dynamic modulus is about 80 percent larger than the statically measured initial tangent modulus.

\section{REFERENCES}

Mullineaux, et al (1965)

"Stratigraphy and Chronology of Late Interglacial and Early Vashon Glacial Time in the Seattle Area, Washington", Geological Survey Bulletin 1194-0. 
$\underline{A P P E N D I X \quad D}$

FILL CONSTRUCTION AND EQUIPMENT INSTALLATION AT FORT LAWTON 
FILL CONSTRUCTION AND EQUIPMENT INSTALLATION

AT FORT LAWTON

A major part of the test program consisted of planning for and constructing an artificial fill to contain detailed instruments for performing the tests and making the evaluations presented in Chapter 5. This set up not only had to duplicate normal in situ test conditions, but preparation of the fill and installation of recording equipment had to be accomplished such that the body of material retained reasonable uniformity.

\section{D-1 FILL CONSTRUCTION}

The initial construction effort consisted of excavating with a rubber-tired tractor-mounted backhoe a large circular pit. The 10-foot deep pit was shaped like a truncated cone, 30 feet in diameter at the ground surface and 20 feet in diameter at the bottom. These overall dimensions were selected to avoid interferences of reflected waves from the boundaries.

During the excavation process removed soils were sorted into two general materials. Top soils and organic materials, as shown in Fig. D-1, were first stripped from the general test area. The remaining materials were removed and temporarily stockpiled as close as possible around the open excavation. This material was later used as backfill. The bottom of the pit was then leveled.

Backfilling operations were achieved in steps. Material was spread in six-inch or less loose lifts with shovels over the entire area of the bottom of the pit, and compacted using two walk-behind vibratory plates. The vibrators, shown in Fig. D-2, weighed 285 and 185 pounds and operated at $5500 \mathrm{rpm}$. 


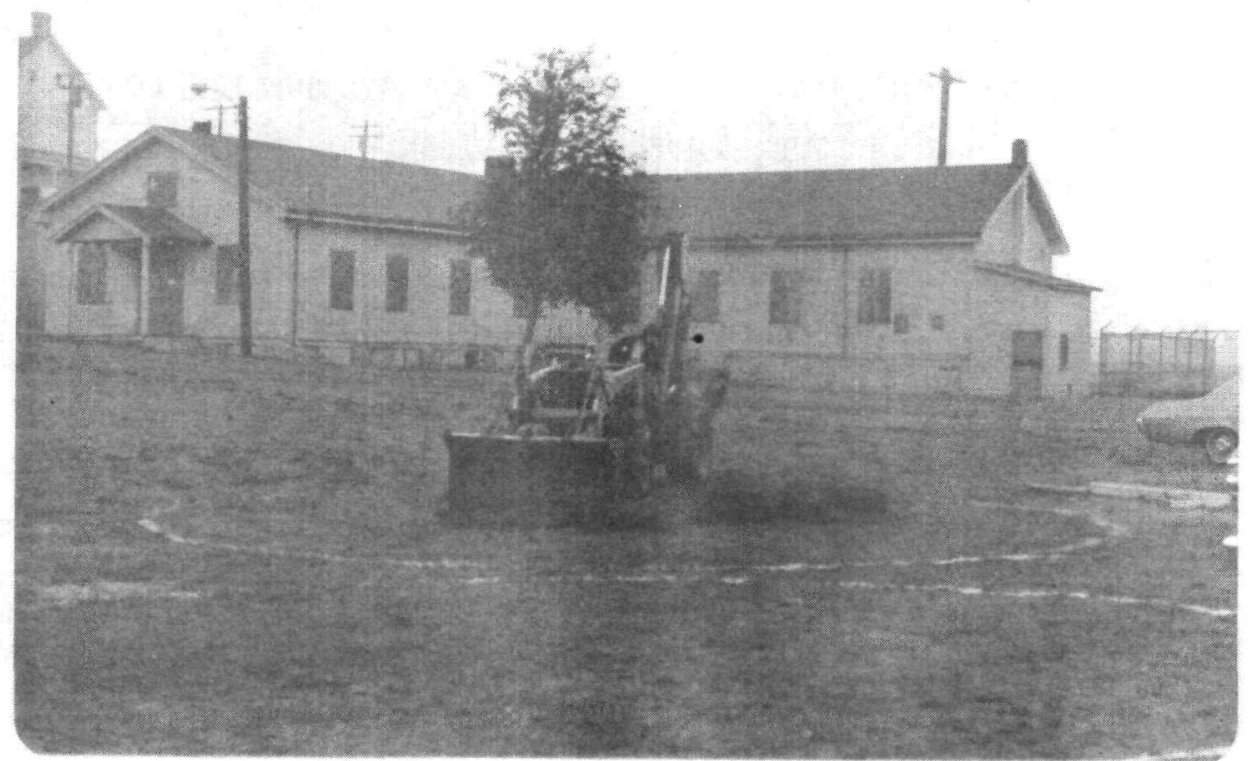

FIGURE D-I INITIATION OF EXCAVATION

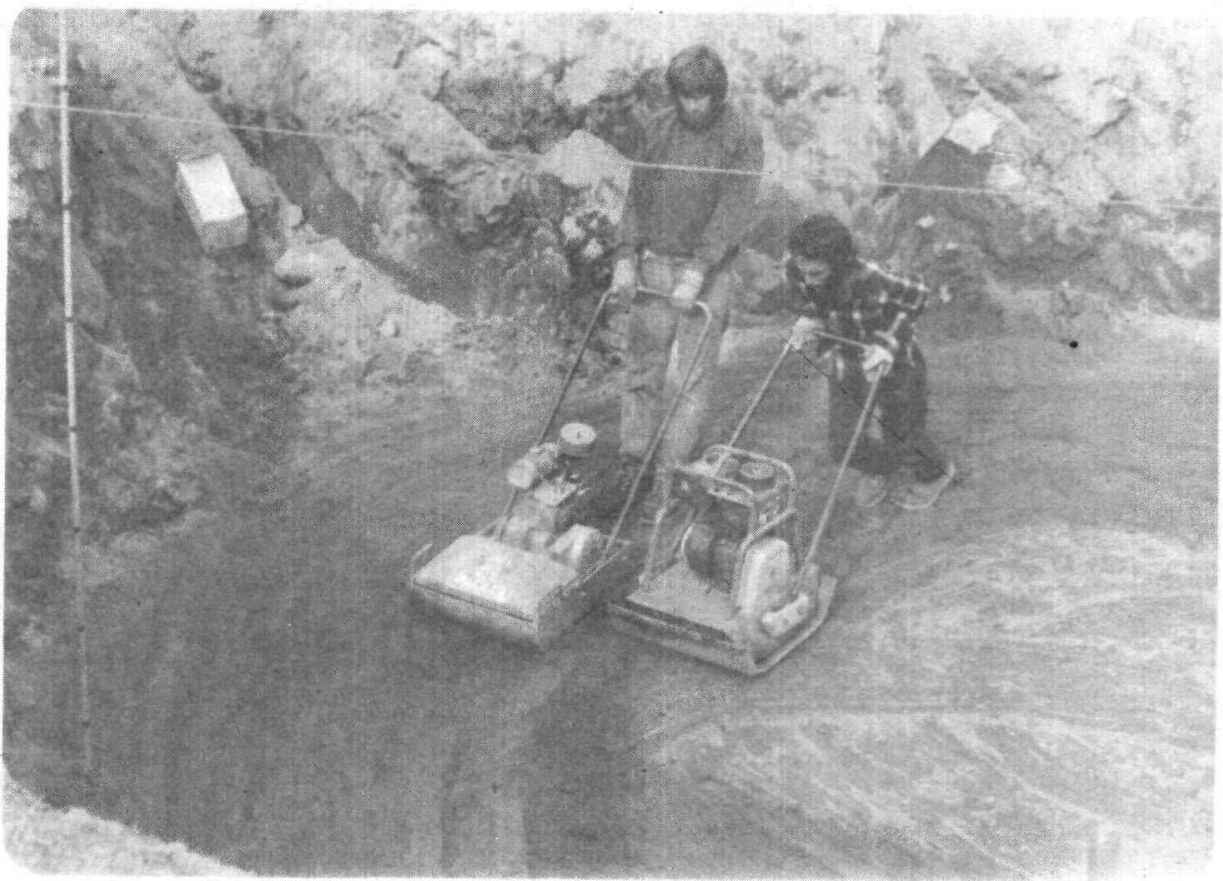

FIGURE D-2 COMPACTION 
All gravel particles larger than 2 inches in diameter were removed by hand and eliminated from the test area. This operation was repeated for the entire depth of the fill.

Compaction was achieved by routing each vibrator in concentric and overlapping circles over the entire pit about three times. Compaction usually started at the outer boundary of the pit and proceeded to the center. The objective of the compacting effort was to obtain a reasonably homogeneous and uniform deposit of soil. A more uniform fill can usually be obtained if the materials are compacted in thin layers to a reasonably dense state.

At the most important depth zone (Elevation - 5 feet) three in place density tests were performed in different parts of the pit to check the uniformity of the deposit. These tests produced densities of $90.4,86.6$ and 81.9 percent of the maximum Modified Proctor density. This corresponds to an average relative density of about 70 percent.

\section{D-2 EQUIPMENT INSTALLATION}

In conjunction with the backfilling operation, equipment was hand placed at predetermined points and eventually covered with subsequent soil layers. The general set up of the special tests in the artificial fill is shown in the Figs. 5- 1 and D-3. The main plane of testing was defined at mid-depth of the fill (Elevation -5 feet).

The generating station, or anchor, was installed in the vertical axis of the pit. During backfilling, this heavy anchor was held in position and aligned vertically by suspending it from a portable tripod. To permit free vertical movement of the anchor and hammering equipment during testing, open cylindrical spaces, protected by one foot diameter corrugated 


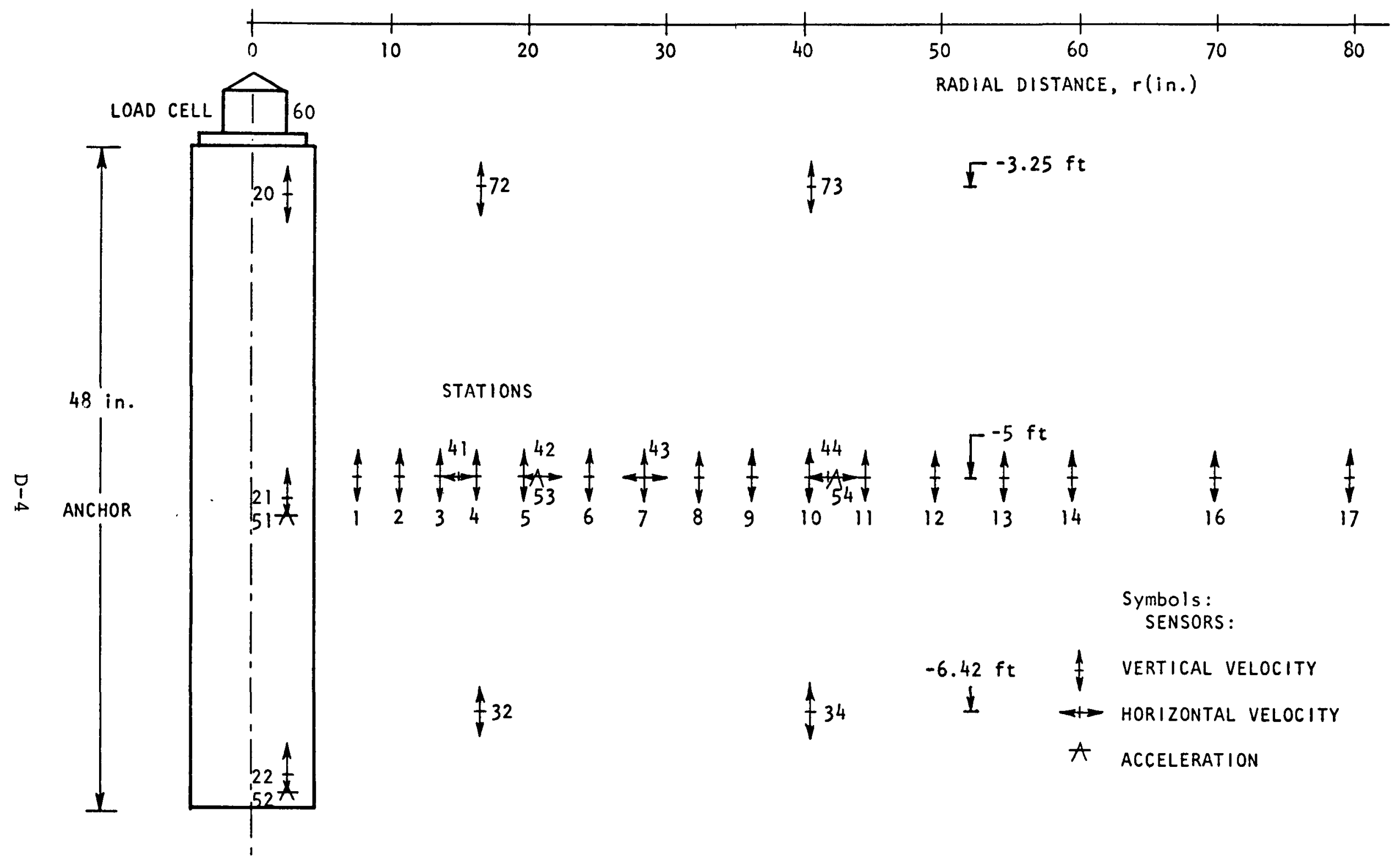

FIG. D-3 INSTRUMENTATION IN ARTIFICIAL FILL 
casing were provided above and below the anchor (Fig. 5-1). This casing was placed to simulate the boundary conditions of the in situ test (i.e., open hole above and below the anchor).

The anchor was instrumented with:

a. A $60 \mathrm{kip}$ load cell, 4,000 hertz natural frequency, $.0252 \mathrm{mV} / \mathrm{lb}$ sensitivity. As shown schematically in Fig. D-3, this load cell was installed on top of the anchor, between the Belleville spring and the striking plate.

b. Three vertically oriented velocity transducers, type Mark Products L-10 AK, 30 hertz natural frequency, $.63 \mathrm{~V} / \mathrm{in} / \mathrm{sec}$ sensitivity. These transducers were firmly fixed in cavities of one plate of the 48 -inch anchor, 3.25, 25.25 and 45.5 inches from the top of the anchor.

c. Two accelerometers, type columbia 200-1, 4,000 hertz natural frequency, $40 \mathrm{mV} / \mathrm{g}$ sensitivity for 9 foot long cable. These sensors were attached to the same leg at distances of 26.5 and 46.75 inches from the top of the anchor.

Figs. D-4 and D-5 show the sensors mounted in the leg of the anchor.

The lower 3-foot long corrugated casing was aligned in position before starting the fill. The fill was then built up around it (Fig. D-6). When the fill reached elevation -7 feet, the anchor was suspended over the casing and aligned to permit free expansion of the anchor, as well as downward slippage, without transmitting direct forces to the casing during the tests. To avoid losses of ground around the anchor plates, 


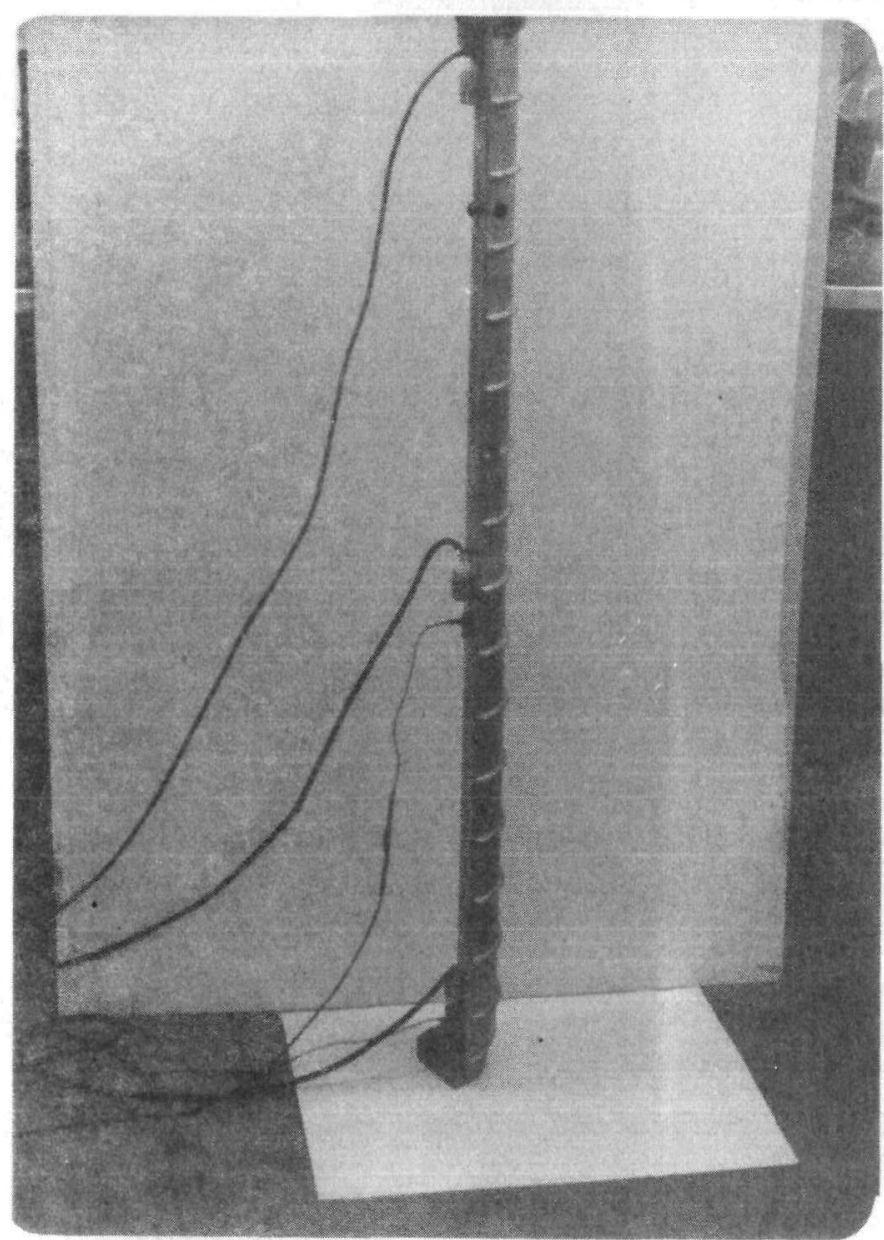

FIGURE D-4

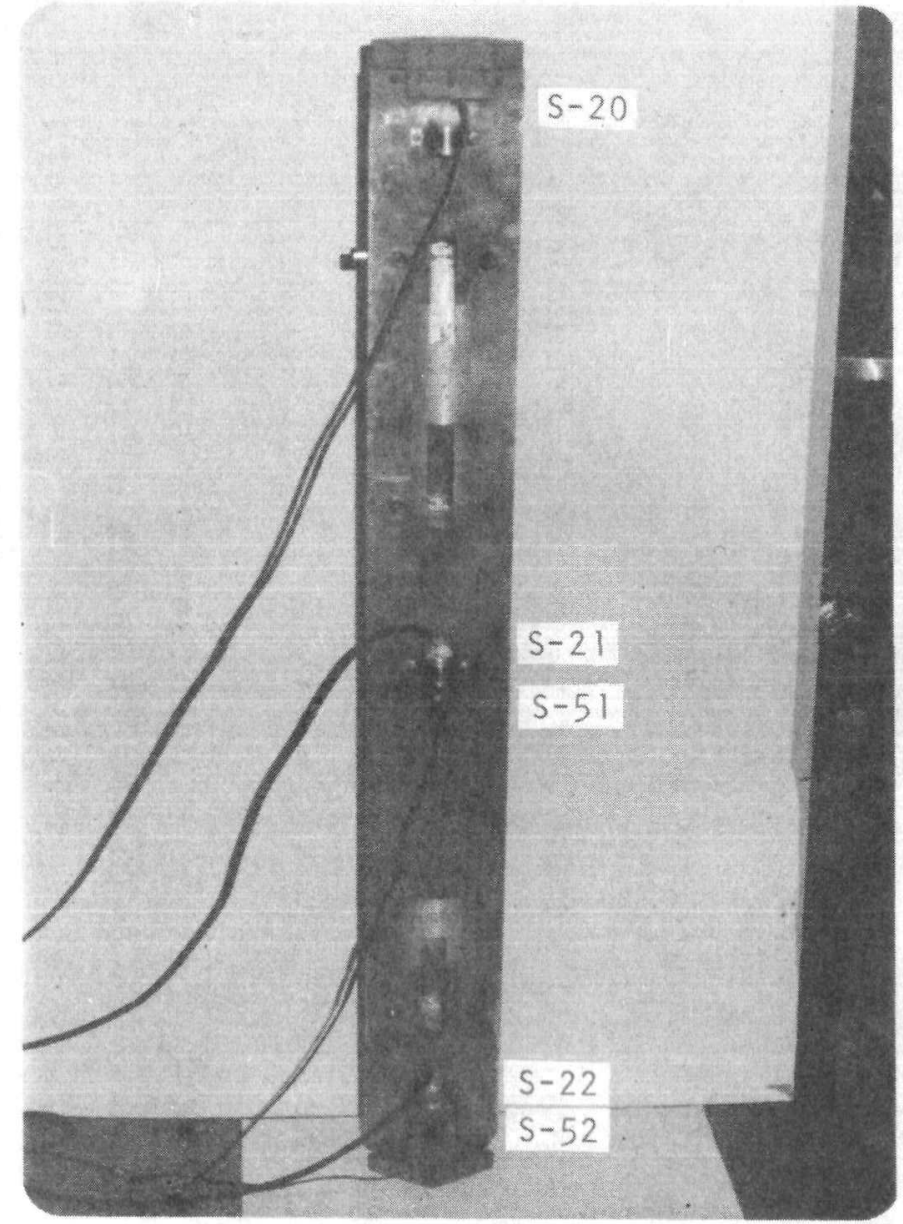

FIGURE D-5

SENSORS IN THE ANCHOR 


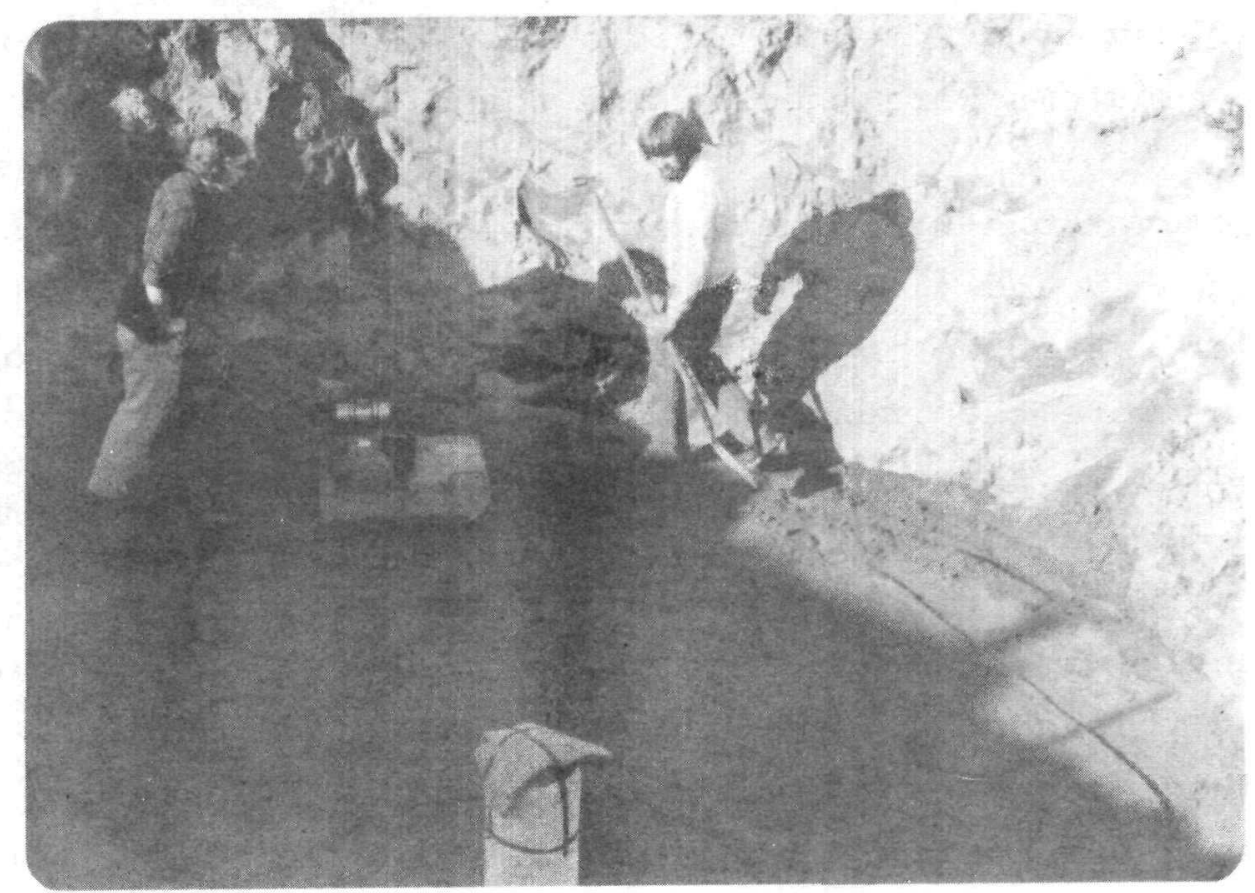

FIGURE D-6 SPREADING AND COMPACTION - FIRST LAYERS 
interior void spaces in the core of the anchor were filled with compressible styrofoam. This styrofoam did not restrict the motions of the anchor and provided the minimum support necessary for the soil to develop arching around the narrow opening between the plates. This same compressible material was also placed at the bottom of the anchor and in the upper part of the lower casing to avoid the displacement of soil around the anchor into the lower cavity. After completion of the tests, both soil retaining systems were checked and found to have behaved adequately.

At elevation -6.75 feet, two vertical velocity transducers were placed 17 and 41 inches, respectively, from the center of the anchor, as shown in Fig. D-7. The fill and compaction operations were then resumed. Fig. D-8 shows construction details at this stage.

Elevation -5 feet was considered the main plane of testing. Consequently, as shown in Figs. $D-3$ and $5-2,33$ sensors were placed at this depth. Of these sensors, two were vertically oriented accelerometers, four were horizontally oriented velocity transducers and the remaining 27 were vertically oriented velocity transducers. When this elevation was reached, the soil along this plane was carefully leveled. A template made from a 1 x 6 wood board was next placed along the axis of the major band of sensors. Finishing nails were then inserted through the holes in the template and pushed into the soil to mark the precise center of each sensor. After this, the template was removed (Fig. D-9). Each nail was then removed and replaced by a transducer as shown in Fig. D-10. Each sensor is shown firmly seated into the soil fill. All sensors installed along this main band are shown in Fig. D-11. These sensors were staggered slightly to avoid interference or shadow effects from adjacent sensors. In general sensors were spaced at least three diameters apart (Appendix E). This staggering also enabled cables to be laid out in a pattern where discontinuity effects would be minimized. 


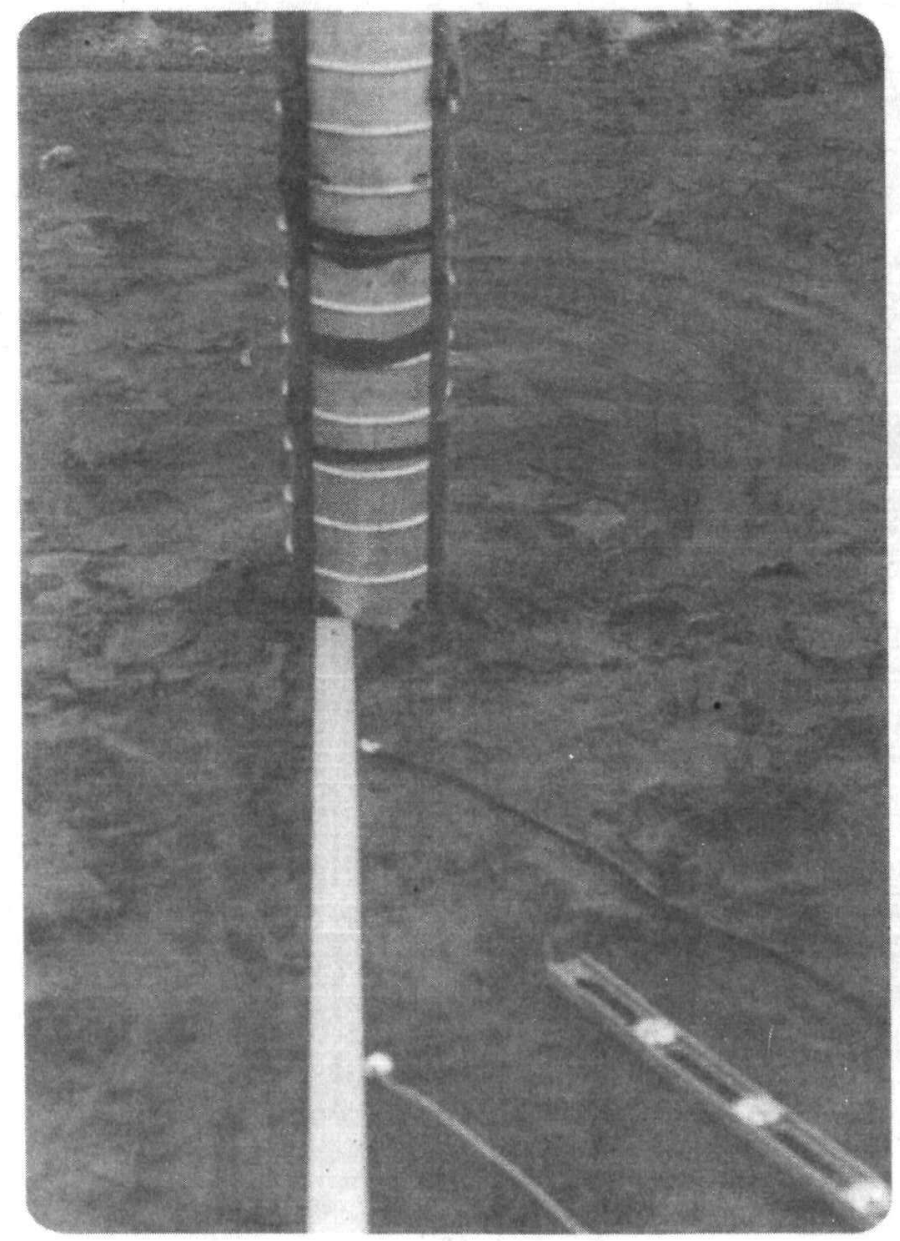

FIGURE D-7 INSTALLATION OF SENSORS AT EL. - 6.75 FT.

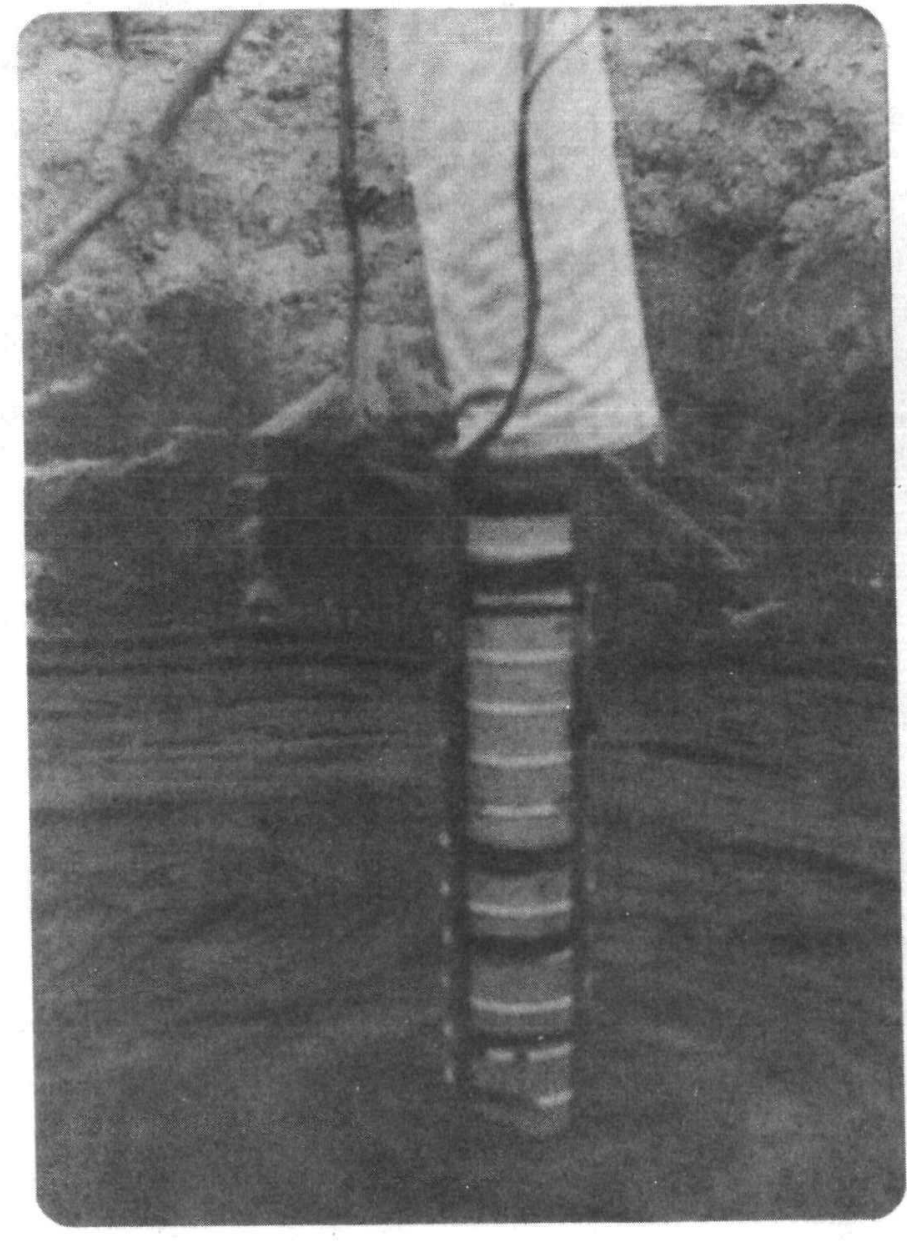

FIGURE D-8 CONSTRUCTION BETWEEN EL. - 6.75 FT. AND EL. - $5.00 \mathrm{FT}$. 


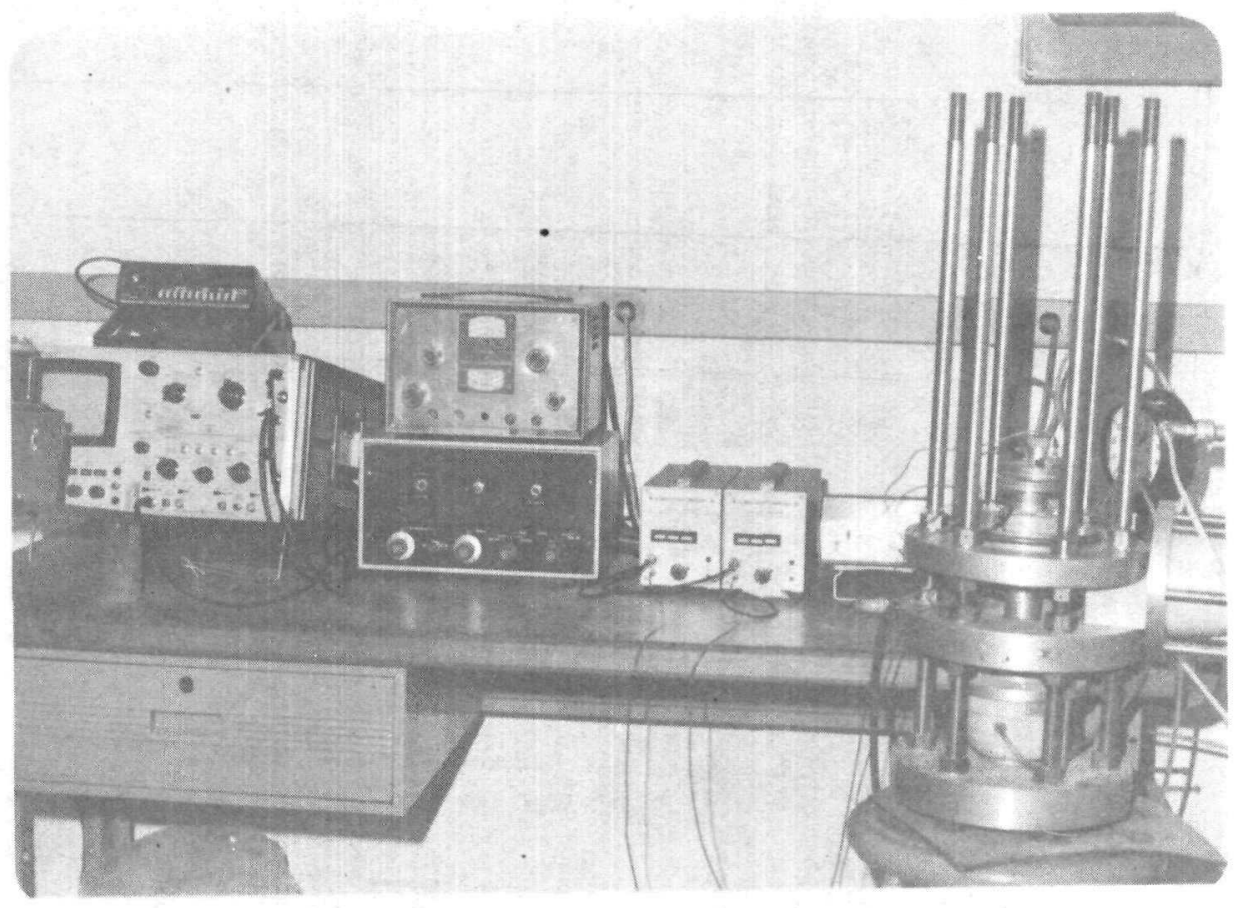

FIGURE D-9 INSTALLATION OF SENSORS AT EL. - 5 FT. 


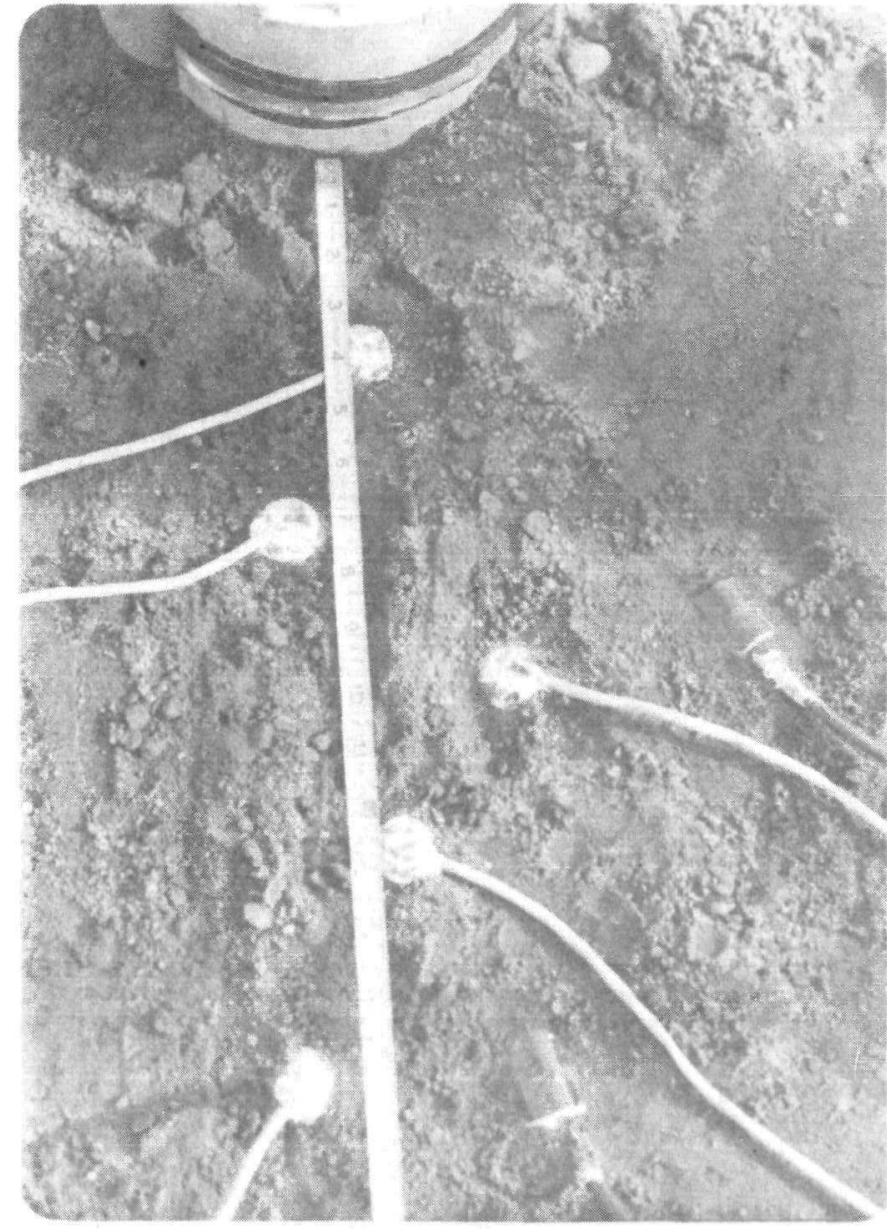

FIGURE D-10

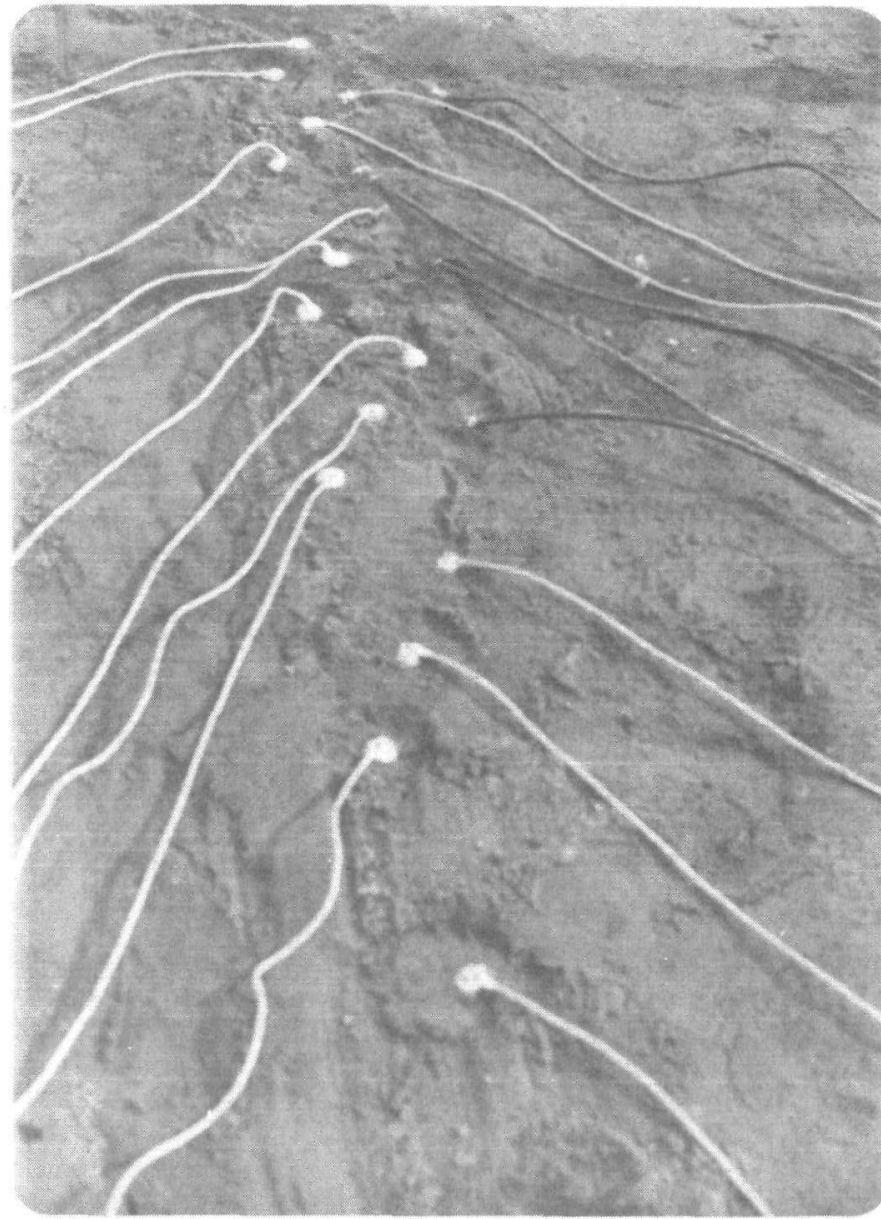

FIGURE D-11

INSTALLATION OF SENSORS AT EL. - 5 FT. 
Other sensors were located and placed using a combination of simple geometric relationships and by taping. Installation procedures for these sensors were essentially the same as described above. All of these free-field sensors were the same types as used in the anchor. The velocity transducers used, depending upon their orientation, could measure either vertical or horizontal motions. They are cylindrical in shape, 1.25 inches in diameter and 1.28 inches long. The accelerometers were positioned to measure only vertical motion. They also are cylindrical units with both lengths and diameters of 0.5 inches.

After all sensors were placed, the distance between the anchor and each sensor was remeasured and recorded. To assure that these distances were correct and that individual sensors had not shifted during subsequent fill compaction, these distances were verified twice by measurements made during and at the completion of the test program. These were accomplished by digging a narrow trench down to the band of instruments and making check measurements. The distances were generally correct to within $1 / 16-$ inch.

Four types of casing were installed down to elevation -6 feet to measure casing effects. This casing was placed vertically and subsequently buried as the backfilling operation continued.

After completion of the installation and measurements of the distances of the sensors, the construction of the fill proceeded in the manner described previously. At elevation -3.25 feet, two vertical velocity transducers were installed at the same distances and in the same vertical plane as the two located at elevation -6.75 feet. These four sensors were to provide information about the characteristics of the motions of the particles and the propagation of wave fronts at different elevations around the anchor. 
The top three feet of the fill was completed after installation of the upper corrugated pipe. This pipe served as protective casing permitting space for the hammer to operate.

In most cases, electrical cables from each transducer were generally run horizontally to the outer boundary of the pit. They were then collected at two main points and run vertically to the ground surface.

The first complete testing phase was accomplished using the large hydraulic anchor assembly shown in most of the photographs. At the completion of testing with this anchor assembly, it was retracted and removed through the upper corrugated casing. The smaller pneumatic anchor was then lowered into the same hole left by the bigger anchor and wedged against the soil face adjacent to the main band of sensors. Soil fill was then poured from the surface through the top corrugated casing around the outside of this anchor to fill the remaining void space. A high density was achieved by tamping each layer of soil in place with a long rod. Additional tests were then conducted with this anchor assembly. At the completion of this testing, all sensors were uncovered, the distance measurements rechecked, and the equipment removed. The results of these tests are discussed in Appendix B. 


\section{APPENDIX E}

TRANSUUCER SPACING CRITERIA FOR SPECIAL TESTS 


\section{APPENDIX E}

TRANSDUCER SPACING CRITERIA FOR SPECIAL TESTS

The objective of this appendix is to discuss the following two items: (1) spacing of transducers in the special tests to avoid interaction effects and (2) conditions by which the relative dynamic motion between the transducers and free field during these tests will be essentially eliminated. Static and dynamic closed-form solutions are applied to develop a criteria for spacing adjacent sensors as closely as possible without distortion of the soil response measurements due to shadow effects.

\section{E-1 INCLUSION RESPONSE TO STATIC LOADS}

The case of a uniaxial tension loading applied to a plate containing a circular hole with an elastic ring insert has been studied by Savin, 1961. The problem, as depicted in Fig. E-1a, can be considered analogous to the placement of transducers in an elastic soil medium, except for Poisson effects.

The circumferential stress concentration for a copper plate was studied for various ring materials and stiffnesses as shown in Fig. E-lb where $H$ is the thickness of the ring and $D=2 R_{1}$. This figure indicates that there is essentially no interaction between the ring and the plate at a distance of 1.5D from the centerline of the ring. Similar conclusions can be made regarding the radial stress concentration factor for a rigid ring insert (Fig. E-lC).

\section{E-2 INCLUSION RESPONSE TO INCIDENT PLANE WAVES}

The response of a rigid spherical inclusion embedded. in an elastic medium subjected to incident plane waves was studied by Mow, 1965, and Bagge, 1973. This study concluded that the medium/inclusion interaction is significant only within that 


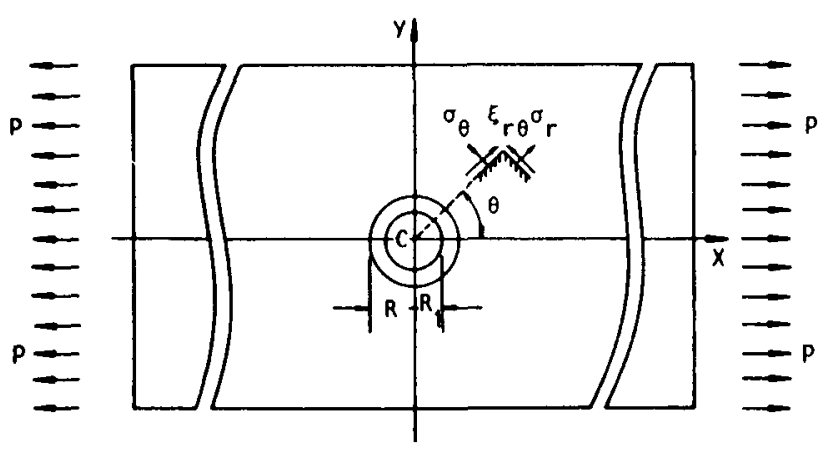

(a) Plate/ring configuration

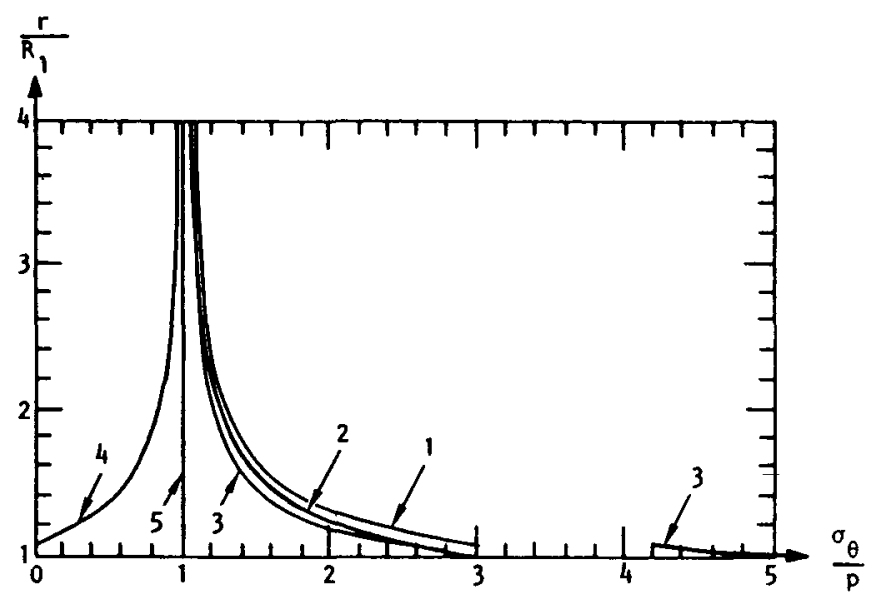

$\alpha=\frac{H}{D}=\frac{1}{30}$

(b) Circumferential stress concentration

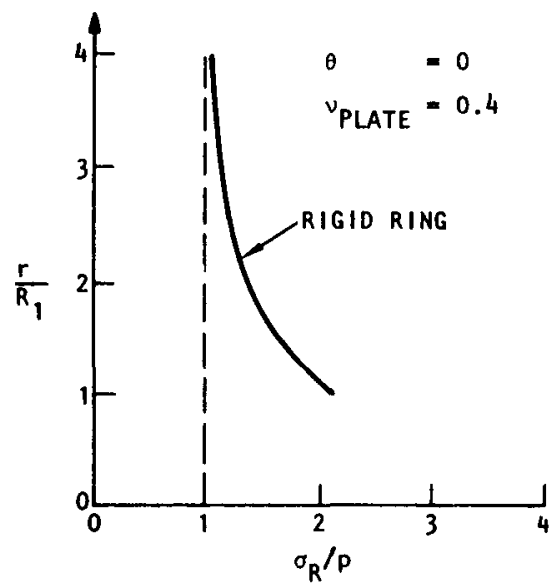

(c) Radial stress concentrations -- rigid ring only

FIGURE E-1. STATIC STRESS CONCENTRATIONS FOR RING INSERT IN AN ELASTIC PLATE ELEMENT 
portion of the medium that lies within a distance of roughly one inclusion diameter from the medium/inclusion interface. This suggests that placement of the transducers at center-center distances of 3D in the in situ tests would eliminate interaction effects between the transducers.

\section{E-3 DYNAMIC INTERACTION OF SOIL/TRANSDUCER SYSTEM}

The dynamic response of rigid spherical inclusions in homogeneous elastic medium subjected to plane waves were studied by Bagge, 1973. The results from this study can be used to investigate dynamic interaction of the soil/transducer system considered in the special tests.

From the study it was concluded that there will be less than 10 percent error in measured response for frequencies that satisfy the condition

$$
\omega<\frac{\pi}{3} \frac{1}{t_{e}}
$$

where $\omega$ is the Erequency of the wave motion in rad/sec and

$$
\begin{aligned}
t_{e}=\mathrm{D} / \mathrm{C} & =\text { engulfment time of the spherical inclusion } \\
\mathrm{D} & =\text { inclusion diameter } \\
\mathrm{C} & =\text { compressional wave velocity of the medium }
\end{aligned}
$$

The cutoff frequency defined by Eq. E.1 is plotted in Fig. E-2 for typical spherical inclusion diameters and effective compressional wave velocities of soils. Since the problem is analogous to the transducer/soil system in the special tests, the studies of Bagge, 1973, can be used to evaluate the degree to which the motion of a transducer might differ from that of the surrounding soil medium. 


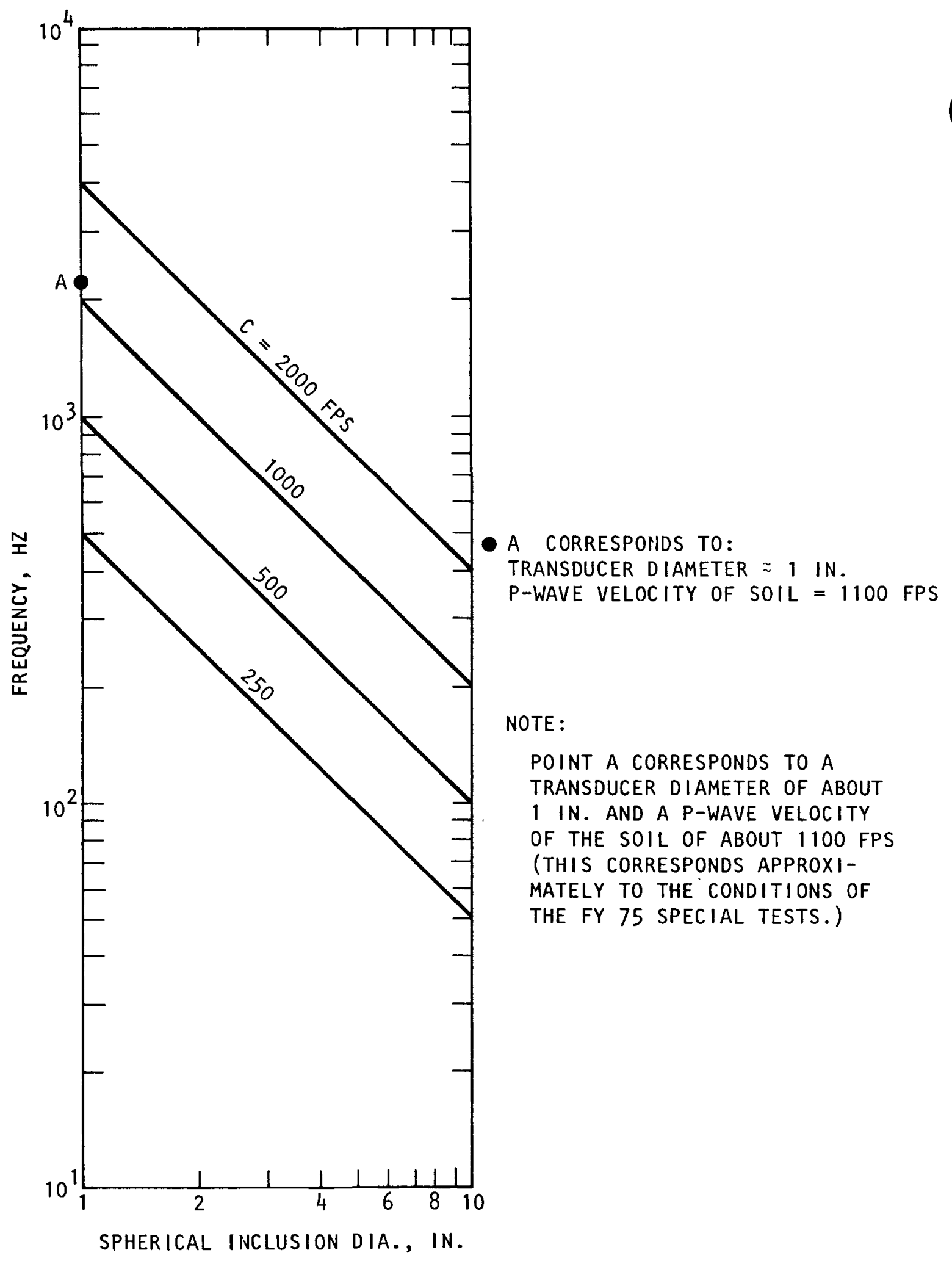

FIGURE E-2. CUTOFF FREQUENCIES BELOW WHICH SOIL/TRANSDUCER (SPHERICAL INCLUSION) INTERACTION HAS MINIMAL EFFECT ON SOIL MOTIONS (< $10 \%$ ERROR) 
Point $A$ in Fig. E-2 corresponds to the diameter of the transducer ( 1 in.) and an estimated P-wave velocity of the soil material of about $1100 \mathrm{fps}$. (This corresponds to an $\mathrm{S}$-wave velocity of $450 \mathrm{fps,}$ a Poisson's ratio of 0.4 , and a density of 110 pcf, which are the approximate properties of the soil material used in the FY75 special tests.) Fig. E-2 indicates that for this case, signals with frequencies above about $2200 \mathrm{~Hz}$ will be affected by interaction effects; while for signals having frequencies below this level, soil/transducer interaction effects will have a negligible effect on the measured soil motions. Prior experience has indicated that the highest measured frequencies in the generated signal will be about 300 to $500 \mathrm{~Hz}$ (SW-AA, 1974); therefore, the results shown in Fig. E-2 indicate that soil/transducer interaction effects will be negligible for the special test program. It is noted that Fig. E-2 is based on the following assumptions: (1) elastic soil medium, (2) rigid transducer, and (3) weight of transducer equivalent to weight of equal volume of soil material.

\section{E-4 CONCLUSIONS AND RECOMMENDATIONS FOR SPECIAL TESTS}

Both static and dynamic studies indicate that interaction between transducers is virtually eliminated at a distance of 1D from interface of the transducer, or a distance of $1.5 \mathrm{D}$ to the center of the transducer. This implies that, in the special tests, the transducers should be spaced at distances not less than $3 \mathrm{D}$ center to center.

Analytical solutions have indicated that for a 1 -inch diameter transducer and a soil medium with a P-wave velocity of $2500 \mathrm{fps}$, the cutoff frequency below which soil/transducer interaction will have minimal effect on free-field soil motions (error < 10\%) is approximately $2200 \mathrm{~Hz}$. Since this frequency is well above the highest significant frequencies expected in the soil response measurements, the relative motion between the 
transducer and the free field should be negligible. Therefore, this information suggests that the use of 1-inch diameter transducers will not cause any significant modification of the shear wave propagation through the soil, during the in situ tests being conducted under the present program.

\section{REFERENCES}

Bagge, C. F. (1973)

"Structure Medium Interaction", R-7243-1-2774, Agbabian Associates, El Segundo, CA, March

Mow, C. C. (1965)

"Transient Response of a Rigid Spherical Inclusion

in an Elastic Medium", Jour. Appl. Mech., September, p. $637-642$

Savin, G. N. (1961)

Stress Concentration Around Holes, translated by Eugene Gros, Permagon Press, New York, p. 249

SW-AA (1974)

"Soil Behavior Under Earthquake Loading Conditions: In Situ Impulse Test for Determination of Shear Modulus for Seismic Response Analyses, Progress Report", A Joint Venture of Shannon \& Wilson, Inc., and Agbabian Associates, June 


\section{APPENDIX F}

S'TATISTICAL ASSESSMENT OF SPECIAL TEST RESULTS 
STATISTICAL ASSESSMENT OF SPECIAL TEST RESULTS

\section{F-1 PURPOSE AND SCOPE}

As described in Chapter 5, a comprehensive special test program has been carried out to evaluate effects of various test parameters on the soil response measured during the in situ test. The test program was conducted in a pit containing a carefully controlled sand fill material within which sensors were hand-placed every few inches apart (Fig. F-l). Soil motions measured at the various sensors were then evaluated as a function of various test parameters associated with the loads applied to the anchor, the anchor jacking pressure, and the placement of the sensors in the soil.

The primary feature of part of this program consists of tests that were repeated a sufficient number of times to permit meaningful statistical evaluations of the resulting ensembles of soil response measurements. These statistical evaluations provide a basis for considering the effects that any scatter in the measurements might have on the trends associated with the above indicated variations in certain test parameters. The purpose of this appendix is to report the results of these statistical evaluations and, from these results, to draw conclusions regarding the effects of the various test parameters on the measured in situ responses of the soil materials.

A large part of the special test program is indicated in Table F-l. From this table, it is seen that the following test variables have been evaluated statistically
a. Repeatabilıty effects
b. Symmetry and shadow effects 


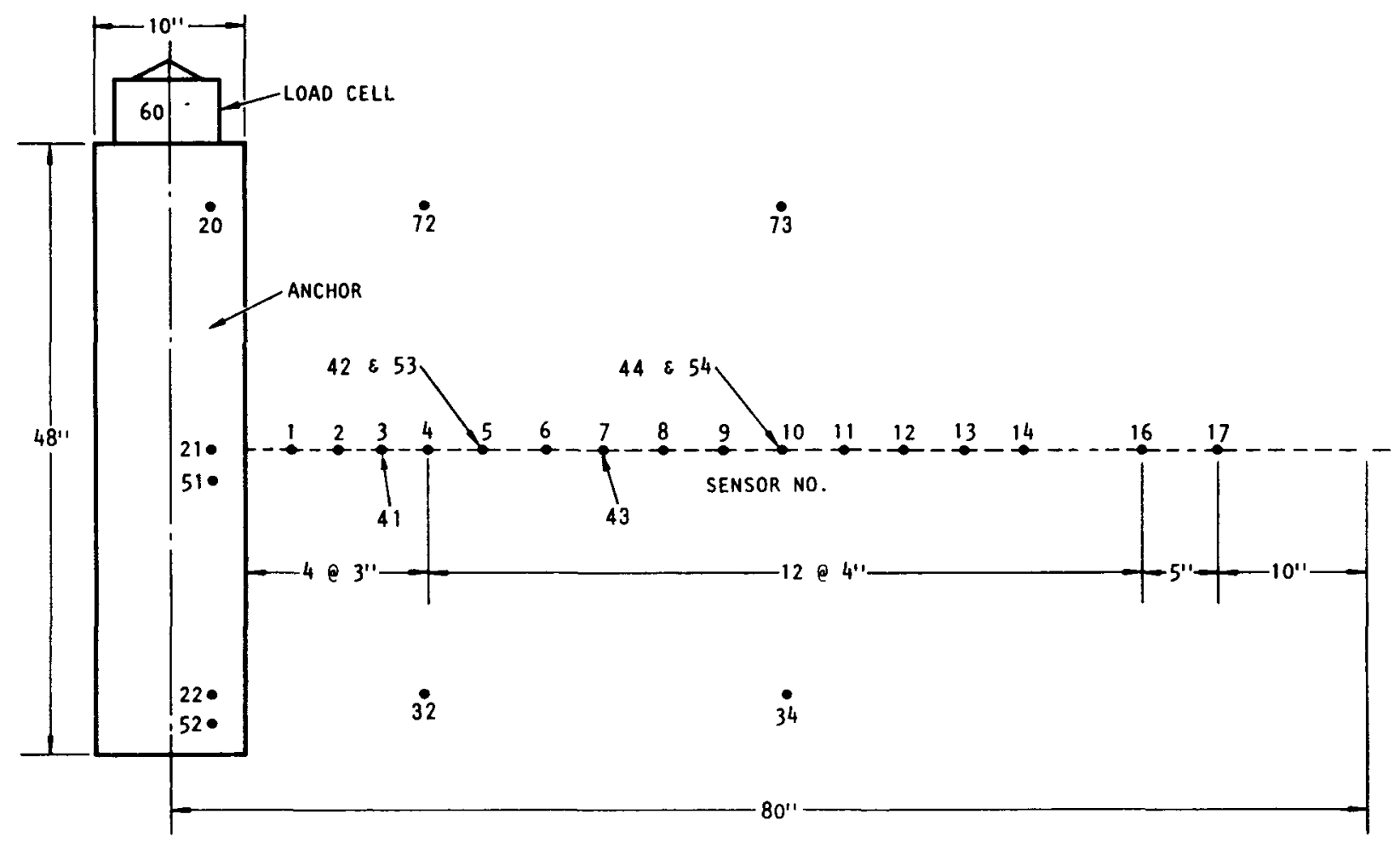

(a) Cross section

NOTE: SENSORS OFFSET RELATIVE TO

AXIS X-X SO THAT THEIR CLEAR

SPACING IS AT LEAST THREE

O FLEXIBLE PIPE

DIAMETERS

- ioa casing efFect stations

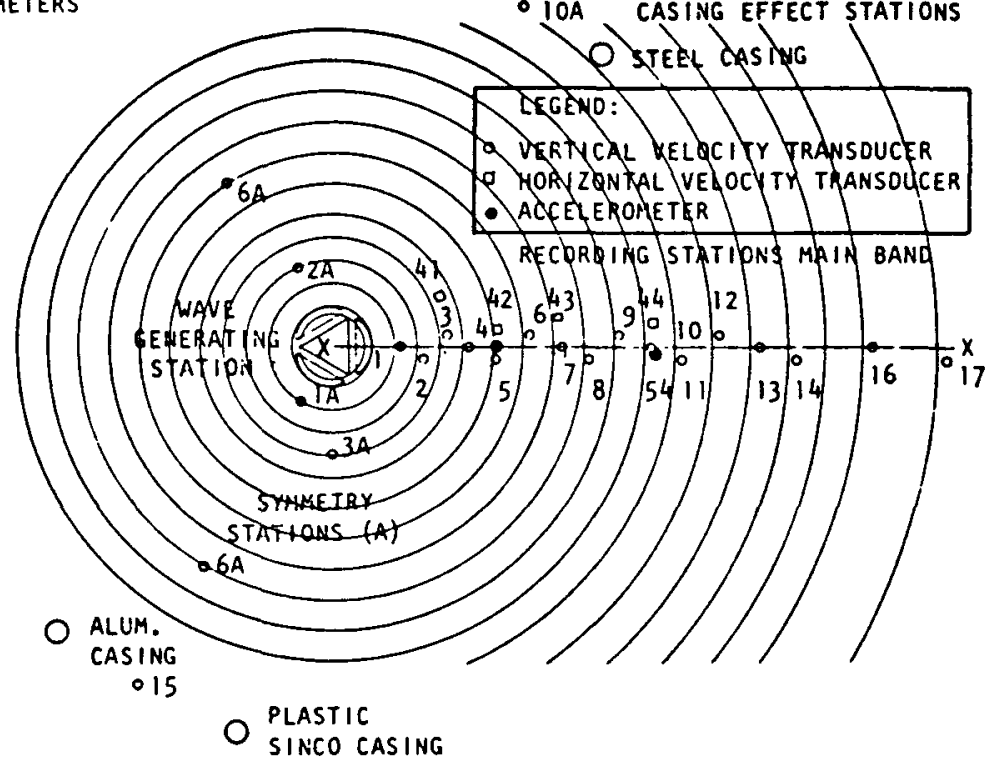

(b) Plan view at elevation $-5 \mathrm{ft}$

FIGURE F-1. LAYOUT OF SENSORS FOR SPECIAL TEST PROGRAM 
TABLE F-1. MEASUREMENTS FOR SPECIAL TEST PROGRAM

\begin{tabular}{|c|c|c|c|c|c|c|c|}
\hline Group & $\begin{array}{c}\text { Case } \\
\text { No. }\end{array}$ & Purpose & Sensors & $\begin{array}{c}\text { Hammer } \\
\text { Weight, Ib }\end{array}$ & $\begin{array}{l}\text { Drop } \\
\text { Height, } \mathrm{ft}\end{array}$ & $\begin{array}{l}\text { Jacking } \\
\text { Pressure } \\
\text { psi }\end{array}$ & $\begin{array}{l}\text { No. of } \\
\text { Repeated } \\
\text { Tests }\end{array}$ \\
\hline$A^{*}$ & $\begin{array}{l}1 \\
2\end{array}$ & Symmetry and Shadow Effects & $\begin{array}{l}1,1 a, 2,2 a \\
3,3 a, 8,8 a\end{array}$ & $\begin{array}{l}58 \\
58\end{array}$ & $\begin{array}{l}0.5 \\
0.5\end{array}$ & 3500 & $\begin{array}{l}12 \\
12\end{array}$ \\
\hline \multirow{5}{*}{$B *$} & $\begin{array}{l}1 \\
2 \\
\end{array}$ & \multirow{5}{*}{$\begin{array}{l}\text { Prior Load History and } \\
\text { Variations in Hammer Weight } \\
\text { and Drop Height }\end{array}$} & $\begin{array}{l}21,3,5,7 \\
7,9,11,14 \\
\end{array}$ & $\begin{array}{r}58 \\
58 \\
-\end{array}$ & $\begin{array}{l}0.5 \\
0.5 \\
\end{array}$ & $\begin{array}{l}3500 \\
3500 \\
\end{array}$ & $\begin{array}{l}12 \\
12 \\
\end{array}$ \\
\hline & $\begin{array}{r}3 \\
4 \\
5\end{array}$ & & $\begin{array}{l}21,1,2,3 \\
4,5,7,8 \\
9,11,12,14\end{array}$ & $\begin{array}{l}58 \\
58 \\
58\end{array}$ & $\begin{array}{r}1.5 \\
1.5 \\
1.5 \\
\end{array}$ & $\begin{array}{l}3500 \\
3500 \\
3500\end{array}$ & $\begin{array}{l}12 \\
12 \\
12 \\
\end{array}$ \\
\hline & $\begin{array}{l}6 \\
7 \\
8 \\
\end{array}$ & & $\begin{array}{l}21,1,2,3 \\
4,5,7,8 \\
9,11,12,14\end{array}$ & $\begin{array}{l}150 \\
150 \\
150 \\
\end{array}$ & $\begin{array}{l}0.58 \\
0.58 \\
0.58 \\
\end{array}$ & $\begin{array}{l}3500 \\
3500 \\
3500\end{array}$ & $\begin{array}{l}12 \\
12 \\
12 \\
\end{array}$ \\
\hline & \multirow{2}{*}{$\begin{array}{c}7 \\
8 \\
9 \\
10 \\
11 \\
12 \\
13 \\
14\end{array}$} & & $\begin{array}{l}21,1,2,3 \\
4,5,7,8 \\
9,11,12,14\end{array}$ & $\begin{array}{l}16.4 \\
16.4 \\
16.4 \\
\end{array}$ & $\begin{array}{l}5.3 \\
5.3 \\
5.3\end{array}$ & $\begin{array}{l}3500 \\
3500 \\
3500\end{array}$ & $\begin{array}{l}12 \\
12 \\
12 \\
\end{array}$ \\
\hline & & & $\begin{array}{l}21,1,2,3 \\
4,5,7,8 \\
9,11,12,14\end{array}$ & $\begin{array}{l}58 \\
58 \\
58\end{array}$ & $\begin{array}{l}0.5 \\
0.5 \\
0.5\end{array}$ & $\begin{array}{l}3500 \\
3500 \\
3500\end{array}$ & $\begin{array}{l}12 \\
12 \\
12\end{array}$ \\
\hline c & $\begin{array}{l}1 \\
2\end{array}$ & P-Wave Effects & $\begin{array}{l}21,41,42,43 \\
21,41,42,43\end{array}$ & $\begin{array}{l}58 \\
58\end{array}$ & $\begin{array}{l}0.5 \\
1.5\end{array}$ & $\begin{array}{l}3500 \\
3500\end{array}$ & $\begin{array}{l}2 \\
2\end{array}$ \\
\hline D & $\begin{array}{l}1 \\
2 \\
3 \\
4\end{array}$ & $\begin{array}{l}\text { Casing Effects } \\
\left(F_{1}, F F_{2}=\text { free-field velocity }\right. \\
\text { transducer between } 71-72 \text { and } \\
73-74 \text {, respectively) }\end{array}$ & $\begin{array}{l}21,71,72, \mathrm{FF}_{1} \\
21,73,74, \mathrm{FF}_{2} \\
21,71,72, \mathrm{FF}_{1} \\
21,73,74, \mathrm{FF}_{2}\end{array}$ & $\begin{array}{l}58 \\
58 \\
58 \\
58\end{array}$ & $\begin{array}{l}0.5 \\
0.5 \\
1.5 \\
1.5\end{array}$ & $\begin{array}{l}3500 \\
3500 \\
3500 \\
3500 \\
\end{array}$ & $\begin{array}{l}2 \\
2 \\
2 \\
2\end{array}$ \\
\hline \multirow{2}{*}{$E^{*}$} & $\begin{array}{r}1 \\
2 \\
-\end{array}$ & \multirow{2}{*}{ Jacking Pressure Effects } & $\begin{array}{l}21,1,2,3 \\
4,5,7,8\end{array}$ & $\begin{array}{l}58 \\
58 \\
\end{array}$ & $\begin{array}{l}0.5 \\
0.5\end{array}$ & $\begin{array}{l}2000 \\
2000 \\
\end{array}$ & $\begin{array}{l}12 \\
12 \\
\end{array}$ \\
\hline & $\begin{array}{l}3 \\
4\end{array}$ & & $\begin{array}{l}21,1,2,3 \\
4,5,7,8\end{array}$ & $\begin{array}{l}58 \\
58\end{array}$ & $\begin{array}{l}0.5 \\
0.5\end{array}$ & $\begin{array}{l}5000 \\
5000\end{array}$ & $\begin{array}{l}12 \\
12\end{array}$ \\
\hline$F$ & $\begin{array}{l}1 \\
2 \\
3 \\
4 \\
5 \\
6\end{array}$ & Anchor Responses & $\begin{array}{ll}60,20,51, & 52 \\
60,20,51, & 52 \\
60,20,51, & 52 \\
60,20,51, & 52 \\
60,20,51, & 52 \\
60,20,51, & 52\end{array}$ & $\begin{array}{r}58 \\
58 \\
150 \\
29 \\
58 \\
58\end{array}$ & $\begin{array}{l}0.5 \\
1.5 \\
0.58 \\
3.0 \\
0.5 \\
0.5\end{array}$ & $\begin{array}{l}3500 \\
3500 \\
3500 \\
3500 \\
2000 \\
5000\end{array}$ & $\begin{array}{l}2 \\
2 \\
2 \\
2 \\
2 \\
2\end{array}$ \\
\hline
\end{tabular}

*Data considered in statistical analysis phase of special test program. 
c. Prior load history

a. Variations in hammer weight and drop height for a given input energy level

e. Jacking pressure

Other test variables indicated in Table F-1, such as casing effects, P-wave effects, and anchor responses have not been evaluated statistically and are therefore not included in this appendix. These tests, together with additional supplemental tests, were performed to study some of these same effects, as well as these other effects, on a non-statistical basis. An evaluation of this data is presented in Appendix G.

The remainder of this appendix is divided into three sections. A description of the data processing approach and analytical procedures used to develop the statistical results is contained in section $\mathrm{F}-2$. These results are described and evaluated in section F-3, while section F-4 contains a summary and conclusions regarding this work.

F-2 DATA PROCESSING APPROACH AND ANALYTICAL PROCEDURES

Prior to presenting the results of the statistical evaluations, it is appropriate to first discuss the approach philosophy associated with the processing of the test data and the analytical procedures used to carry out this processing. This discussion is contained in the remaining paragraphs of this section.

\section{F-2.1 Data Processing Approach}

As noted in Table $\mathrm{F}-1$, ensembles of repeated soil response measurements were obtained at a large number of the sensors used in the special tests. Upon examination of these measurements, it was judged appropriate to carry out statistical processing of the measurements at selected sensors that represent 
the soil response at different ranges of distances from the anchor. Measurements at the remaining sensors could then be used as backup, in case additional investigation of the trends related to the various test parameters was required. Therefore, to assess symmetry and shadow effects, soil responses measured at Sensors $1,2,3$, and 8 were processed. The repeatability assessments and evaluations of the effects of prior load history, hammer weight-drop height variations and jacking pressures were carried out by processing response measurements at sensors 21 , 1, 3, 7, and 14. Locations of these various sensors relative to the anchor and a complete summary of the data processed in this statistical evaluation are provided in Table F-2.

The results from the repeatability assessments are presented in the form of overlaid plots of velocity time histories. Results from the assessments of the other test variables are provided as mean velocity time histories and in addition, as velocity history bands that represent mean + one standard deviation (MSD) bounds. The mean time histories indicate the general trends associated with the effects of each test variable, while the MSD bounds indicate to what extent these trends might be affected by scatter in the data. It is noted that the probability level associated with these MSD bounds is dependent on the assumed probability distribution that can be associated with the in situ soil tests. For example, if the data follows a normal (Gaussian) distribution, then $68 \%$ of the total population of velocity histories will fall within the MSD bounds. Other probability distributions will result in a slightly different estimate of this percentage of the total population of test results contained within these MSD limits.

\section{F-2.2 Analytical procedure}

Two processing packages were used in evaluating this data. The first was a specially written interface program that 
TABLE F-2. DATA PROCESSED IN STATISTICAL EVALUATIONS

\begin{tabular}{|c|c|c|c|}
\hline $\begin{array}{l}\text { Sensor } \\
\text { No. }\end{array}$ & $\begin{array}{c}\text { Distance from Edge } \\
\text { of Anchor, In. }\end{array}$ & Effect & Tests to Compare \\
\hline 21 & At Anchor & $\begin{array}{l}\text { Repeatability } \\
\text { Prior Load History } \\
\text { Different Combinations of Drop } \\
\text { Height and Hammer Weight for } \\
\text { Same Energy Level } \\
\text { Jacking Pressure }\end{array}$ & $\begin{array}{l}\text { Selected Tests } \\
B-1, B-12 \\
B-3, B-6, B-9 \\
B-12, E-1, E-3\end{array}$ \\
\hline $\begin{array}{c}1 \\
1 A\end{array}$ & $\begin{array}{l}2.94 \\
2.43\end{array}$ & $\begin{array}{l}\text { Repeatability } \\
\text { Symmetry and Shadow Effects } \\
\text { Prior Load History } \\
\text { Different Combinations of Drop } \\
\text { Height and Hammer Weight for } \\
\text { Same Energy Level } \\
\text { Jacking Pressure }\end{array}$ & $\begin{array}{l}\text { Selected Tests } \\
\text { A-1 } \\
A-1, B-12 \\
B-3, B-6, B-9 \\
B-12, E-1, E-3\end{array}$ \\
\hline $\begin{array}{c}2 \\
2 A\end{array}$ & $\begin{array}{l}6.13 \\
5.07\end{array}$ & Symmetry and Shadow Effects & $A-1$ \\
\hline $\begin{array}{c}3 \\
3 A\end{array}$ & $\begin{array}{l}9.13 \\
8.5\end{array}$ & $\begin{array}{l}\text { Repeatability } \\
\text { Symmetry and Shadow Effects } \\
\text { Prior Load History } \\
\text { Different Combinations of Drop } \\
\text { Height and Hammer Weight for } \\
\text { Same Energy Level } \\
\text { Jacking Pressure }\end{array}$ & $\begin{array}{l}\text { Selected Tests } \\
A-2 \\
A-2, B-12 \\
B-3, B-6, B-9 \\
B-12, E-1, E-3\end{array}$ \\
\hline 7 & 23.93 & $\begin{array}{l}\text { Repeatability } \\
\text { Prior Load History } \\
\text { Different Combinations of Drop } \\
\text { Height and Hammer Weight for } \\
\text { Same Energy Level } \\
\text { Jacking Pressure }\end{array}$ & $\begin{array}{l}\text { Selected Tests } \\
B-1, B-13 \\
B-5, B-7, B-10 \\
B-13, E-2, E-4\end{array}$ \\
\hline $\begin{array}{l}8 \\
8 A\end{array}$ & $\begin{array}{l}27.86 \\
27.79\end{array}$ & Symmetry and Shadow Effects & $A-2$ \\
\hline 14 & 55.11 & $\begin{array}{l}\text { Repeatability } \\
\text { Prior Load History } \\
\text { Different Combinations of Drop } \\
\text { Height and Hammer Weight for } \\
\text { Same Energy Level }\end{array}$ & $\begin{array}{l}\text { Selected Tests } \\
8-2,8-14 \\
B-5, B-8, B-11\end{array}$ \\
\hline
\end{tabular}


carried out the following initial data reduction and formatting operation :

\author{
a. Read the magnetic tapes containing the \\ field data \\ b. Scale the velocity data using factors pro- \\ vided in the tape label record that precedes \\ each test \\ c. Integrate the velocity time histories, where \\ required \\ d. Label the test channels \\ e. Write a new data tape according to a format \\ suitable for direct input into MAC/RAN
}

The second processing package used in this study was MAC/RAN, a comprehensive system of digital computer programs for complete reduction of time series data. MAC/RAN was applied in the special test program in two different ways. First, to assess the repeatability of the data, velocity time histories measured at each sensor and corresponding to repeated applications of each test condition were multiple-plotted. In this, ten of the twelve repeated tests for each case (Table F-l) were utilized. The scaled data from the interface program were merged to form files of data that were grouped by sensor number rather than test number. These files were then multiple-plotted using consistent amplitude and time scales, so that the repeatability could be easily assessed.

In addition to multiple-plotting of the individual velocity history measurements, MAC/RAN was also used to perform statistical analyses of the ensembles of data. In this, ten data channels for each selected sensor and each test condition were processed to obtain velocity time histories corresponding to the ensemble mean and the ensemble MSD bounds. The multipleplot capabilities of MAC/RAN were then used to overlay the 
time histories corresponding to different variations of each test parameter.

\section{F-3 RESULTS OF STATISTICAL ASSESSMENTS}

The results of the statistical assessment of the special test results are described and evaluated in this section. The section is organized into separate subsections that consider the repeatability assessment, and the evaluation of symmetry and shadow effects, the effects of prior load history, hammer weightdrop height combinations, and jacking pressures.

\section{F-3.1 Repeatability Assessment}

Assessments of the repeatability of velocity histories recorded at the various sensors indicated in Table $\mathrm{F}-2$ are summarized in Figs. F-2 to F-6. These figures indicate that, on the whole, the velocity measurements are very repeatable. The only clear exception to this general trend is the results corresponding to the 150-1b hammex drop (Test Groups B-6 to B-8 in Table $F-1$ ). For this case, the velocity measurements obtained at or near the anchor appear to have a relatively high degree of variability; however, for sensors located at increased distances from the anchor, the variability of the measurements diminishes substantially and the results become quite repeatable. This overall trend may be attributed to the sensitivity of the nearanchor measurements to the details of the applied loadings, such as the orientation of the hammer as it strikes the Belleville springs, the manner in which the anchor plates bear against the sides of the borehole, and the ability to manually obtain the same exact drop repeatedly. The soil response at sensors located away from the immediate vicinity of the anchor tends to be less sensitive to such load details. 


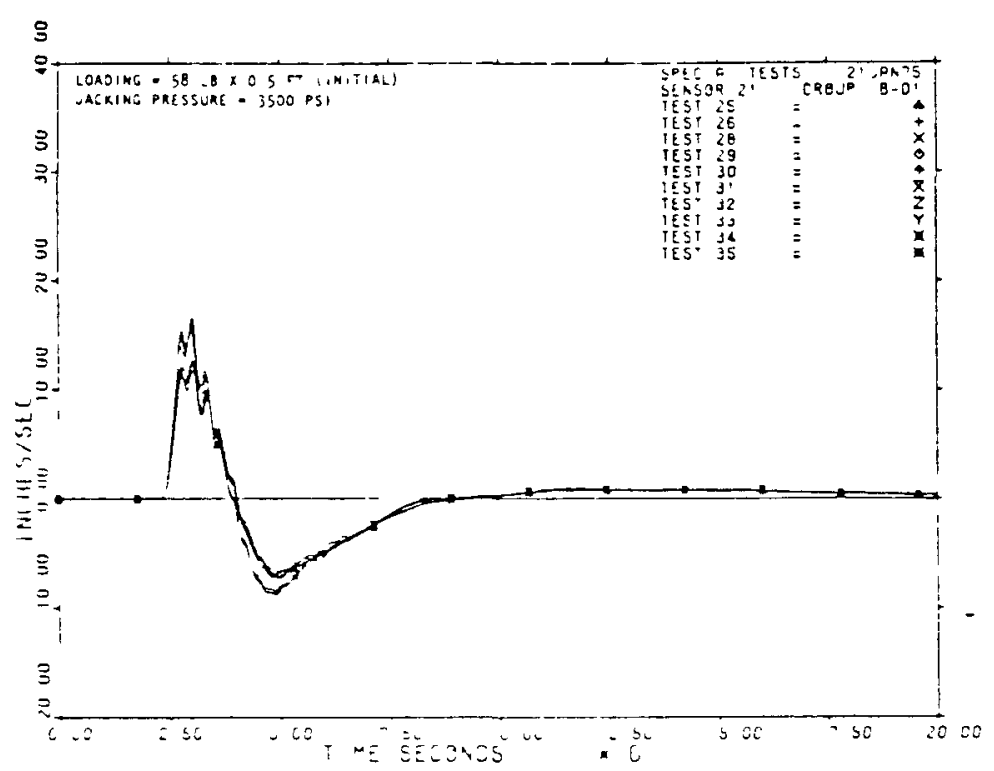

1 (a) Test Group 8-1

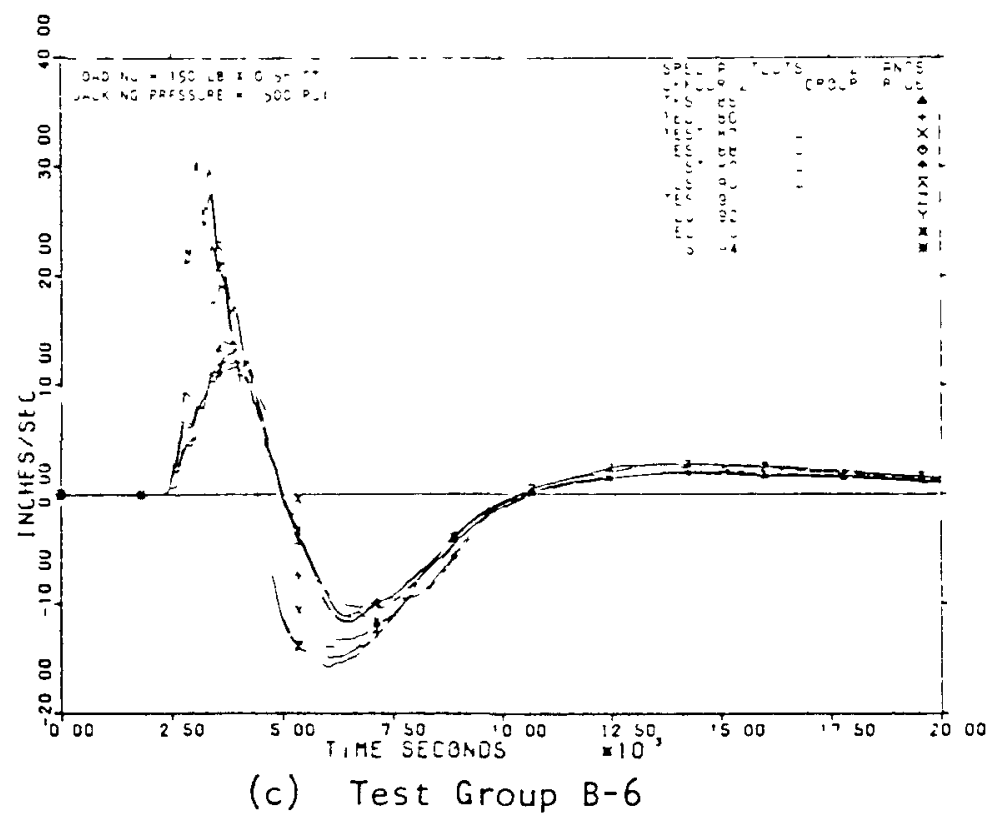

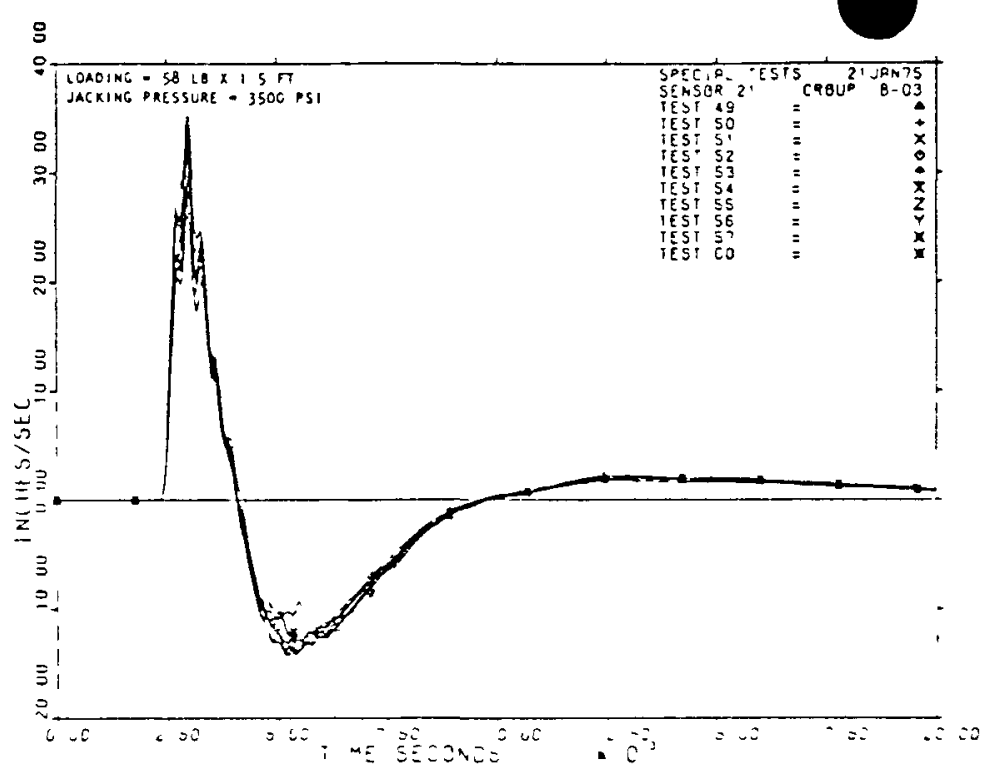

(b) Test Group B-3

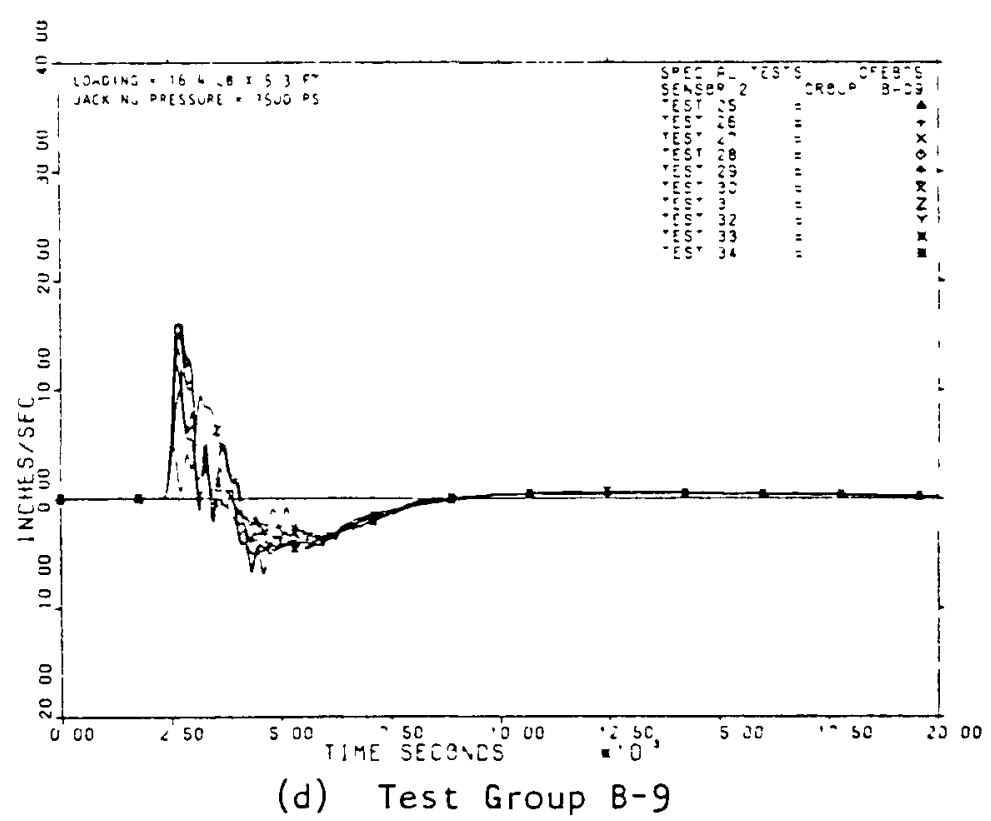

FIGURE F-2. REPEATABILITY OF VELOCITY HISTORIES AT ANCHOR 

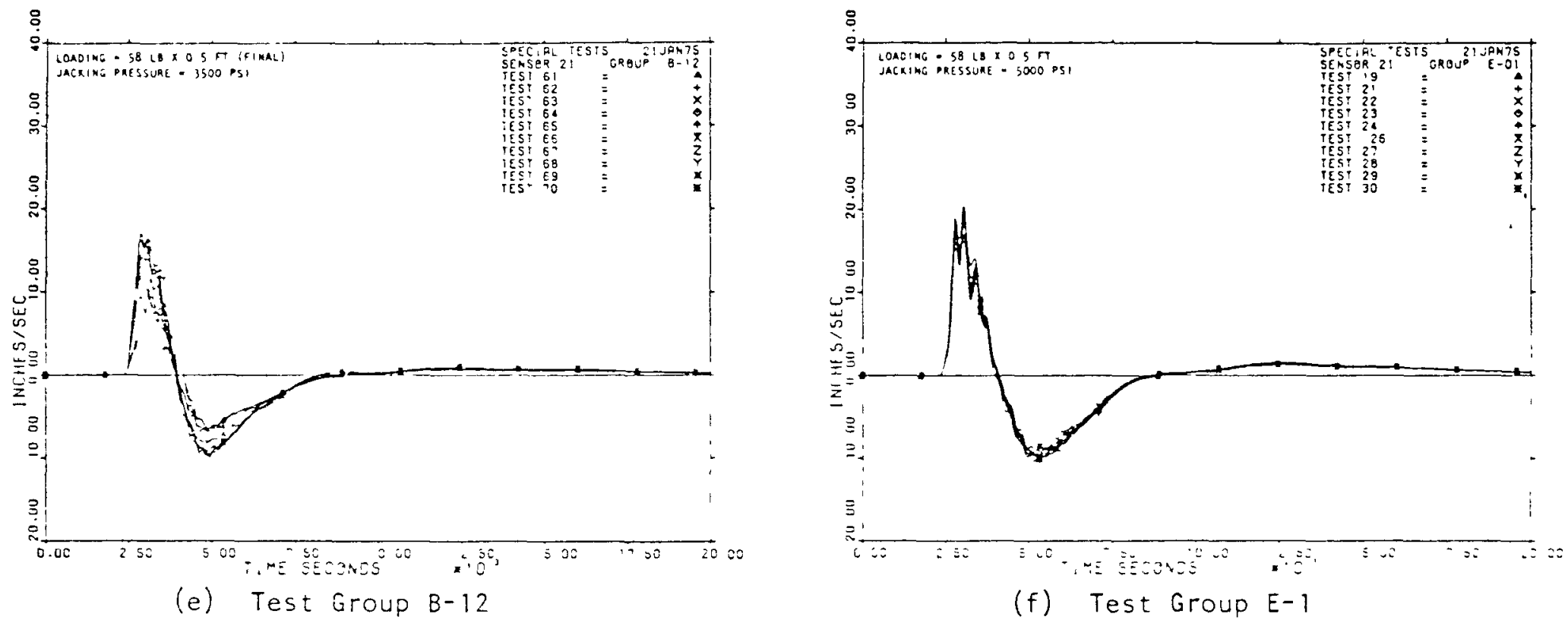

羿

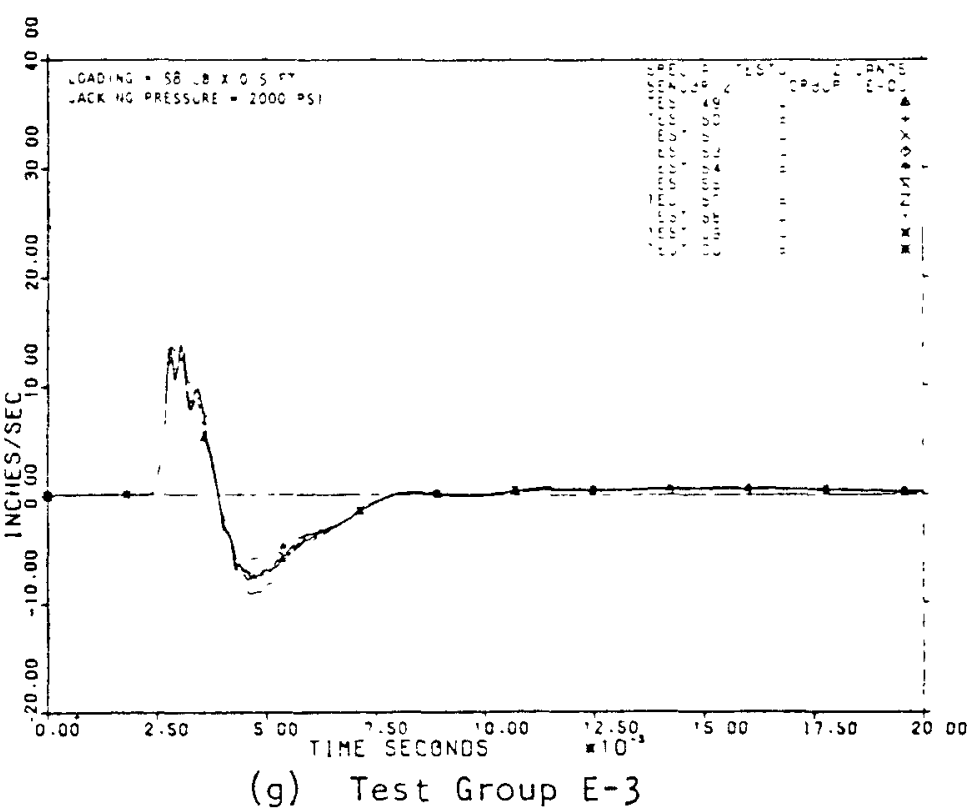

FIGURE F-2. (CONCLUDED) 


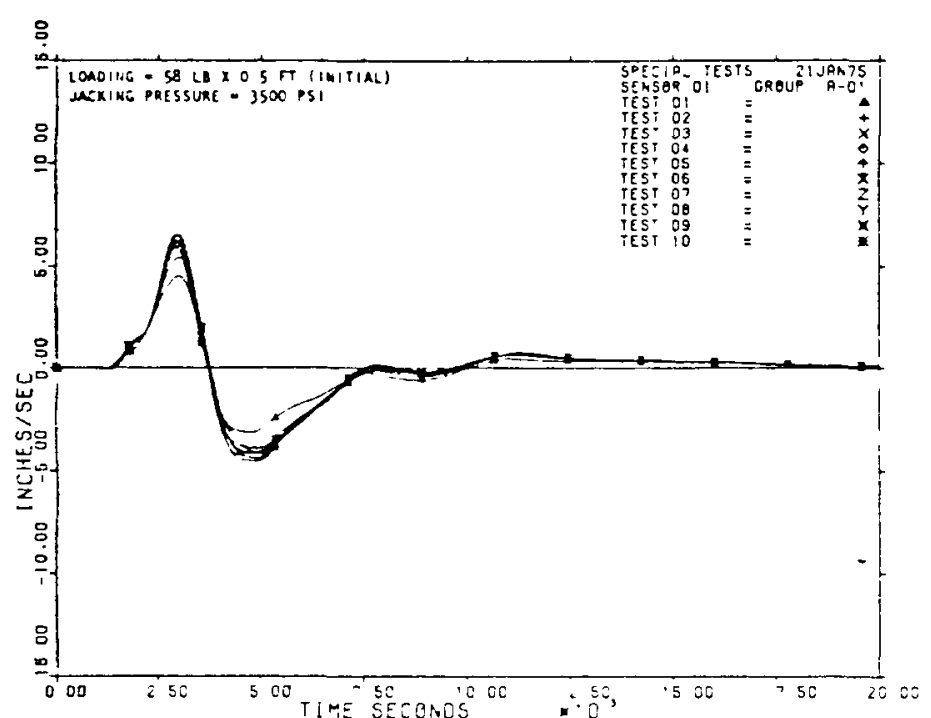

(a) Test Group B-1

$\stackrel{n}{1}$

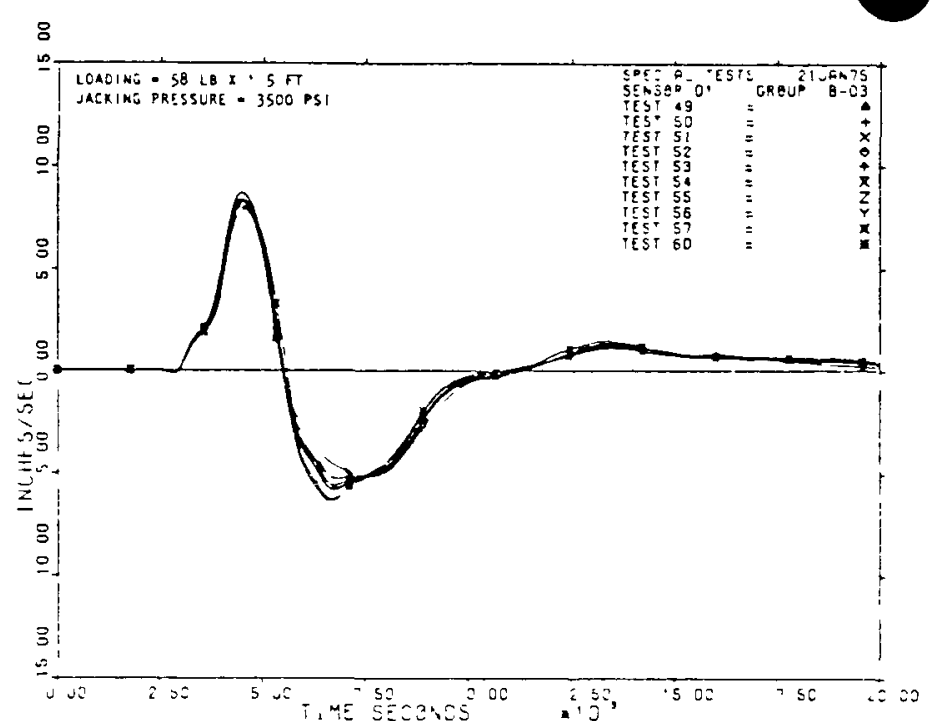

(b) Test Group B-3

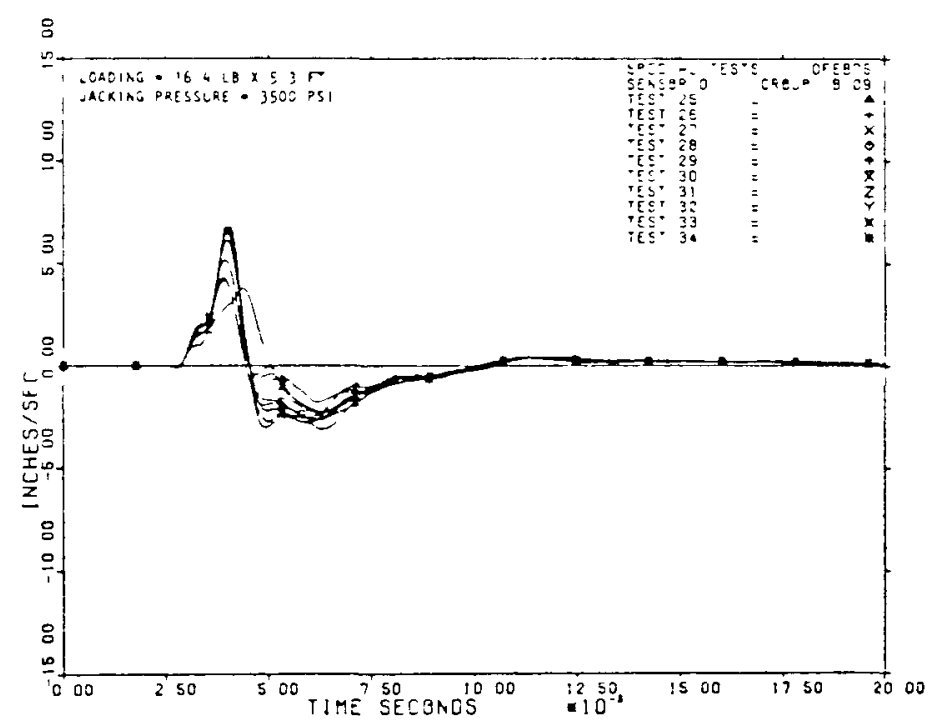

(d) Test Group B-9

FIGURE F-3. REPEATABILITY OF VELOCITY HISTORIES AT SENSOR 1 -- 3 IN. FROM ANCHOR EDGE 


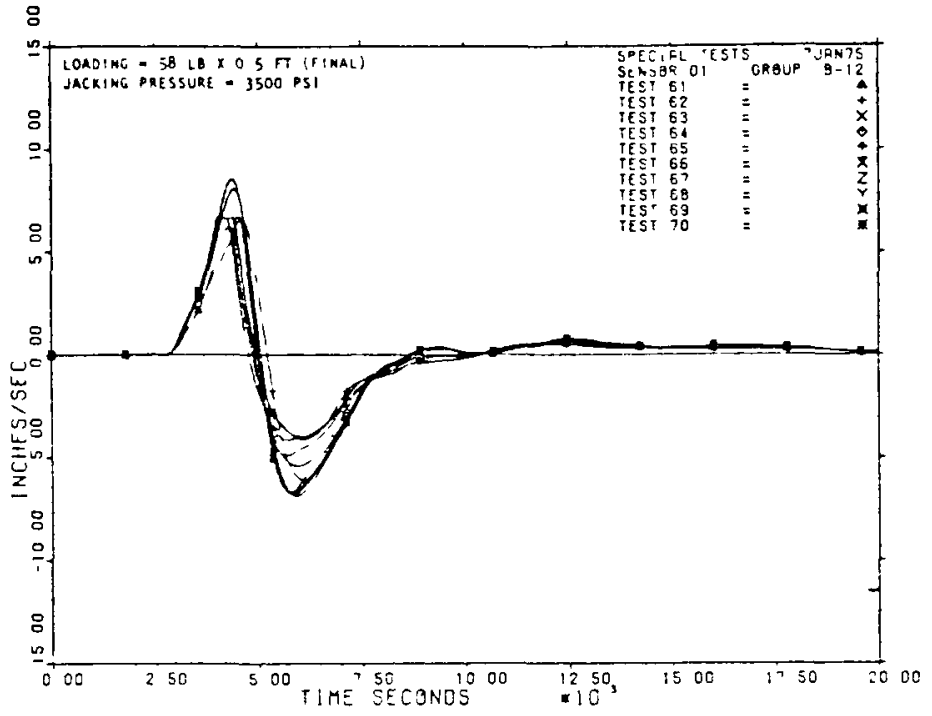

(e) Test Group B-12

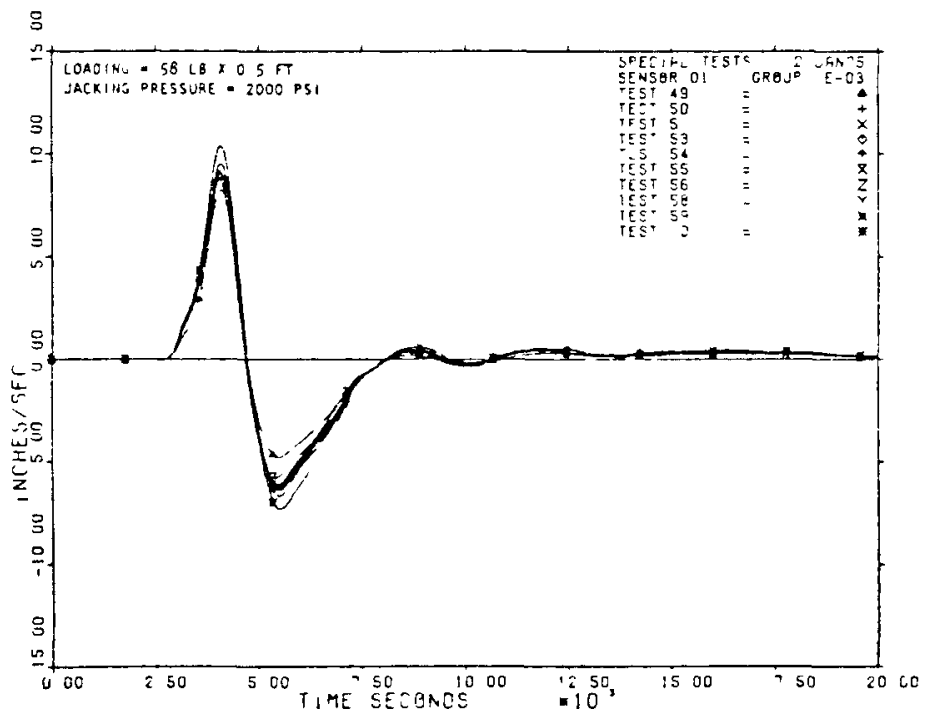

(g) Test Group E-3

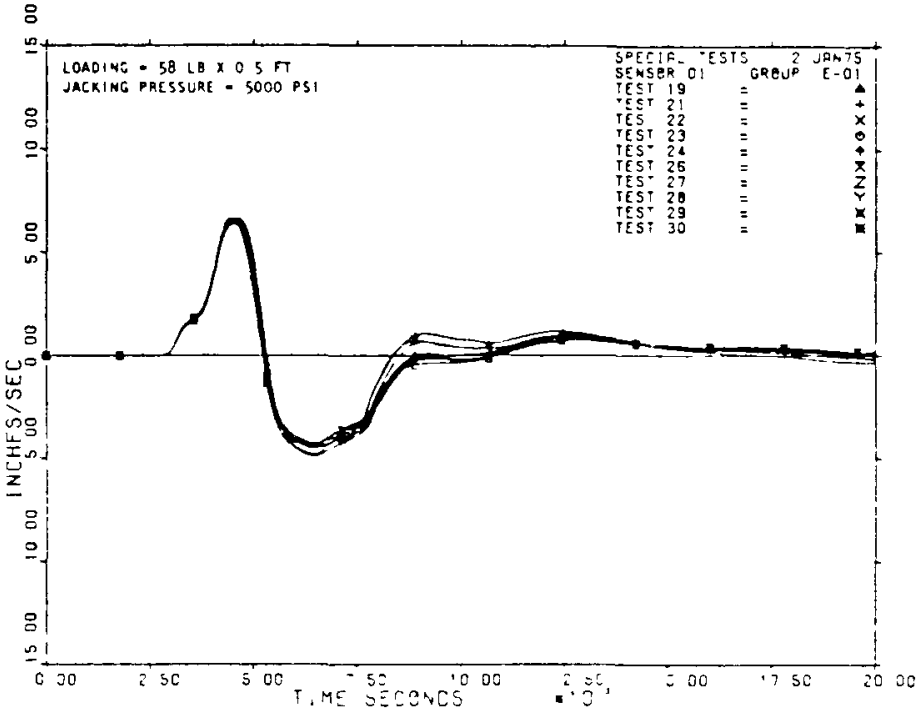

(f) Test Group E-1 


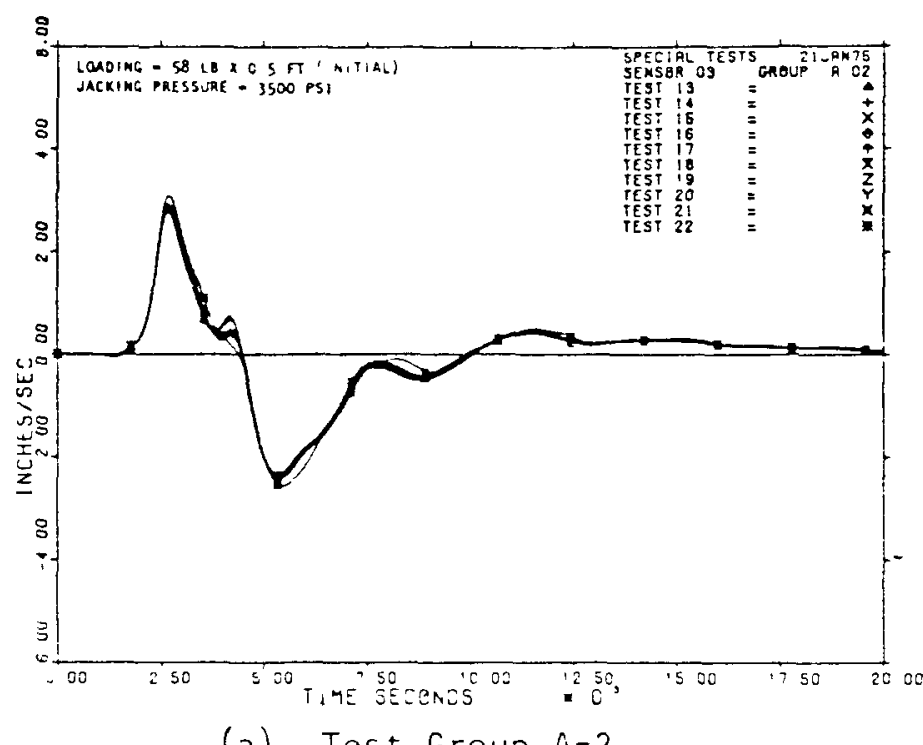

w

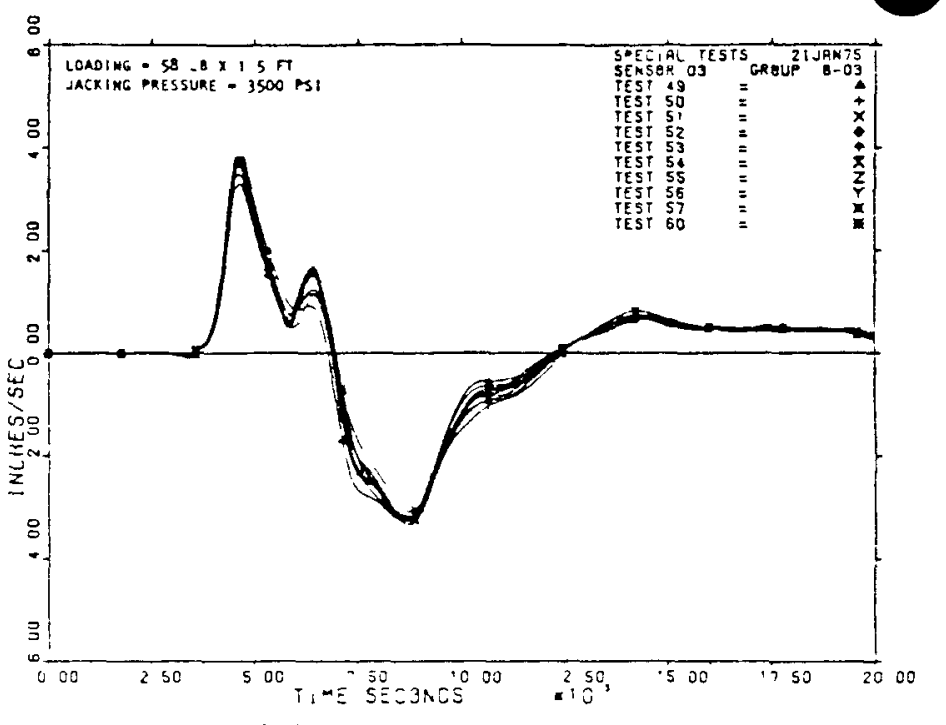

(b) Test Group B-3
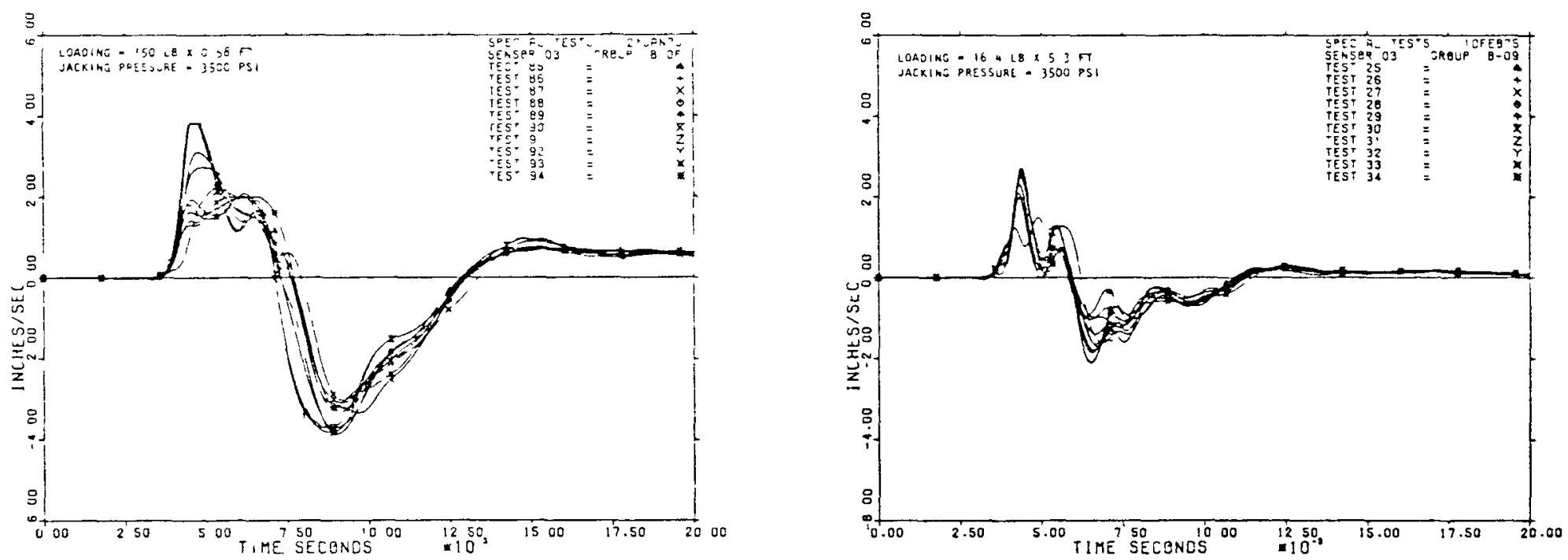

(d) Test Group B-9

FIGURE F-4. REPEATABILITY OF VELOCITY HISTORIES AT SENSOR 3 -- 9 IN. FROM ANCHOR EDGE 

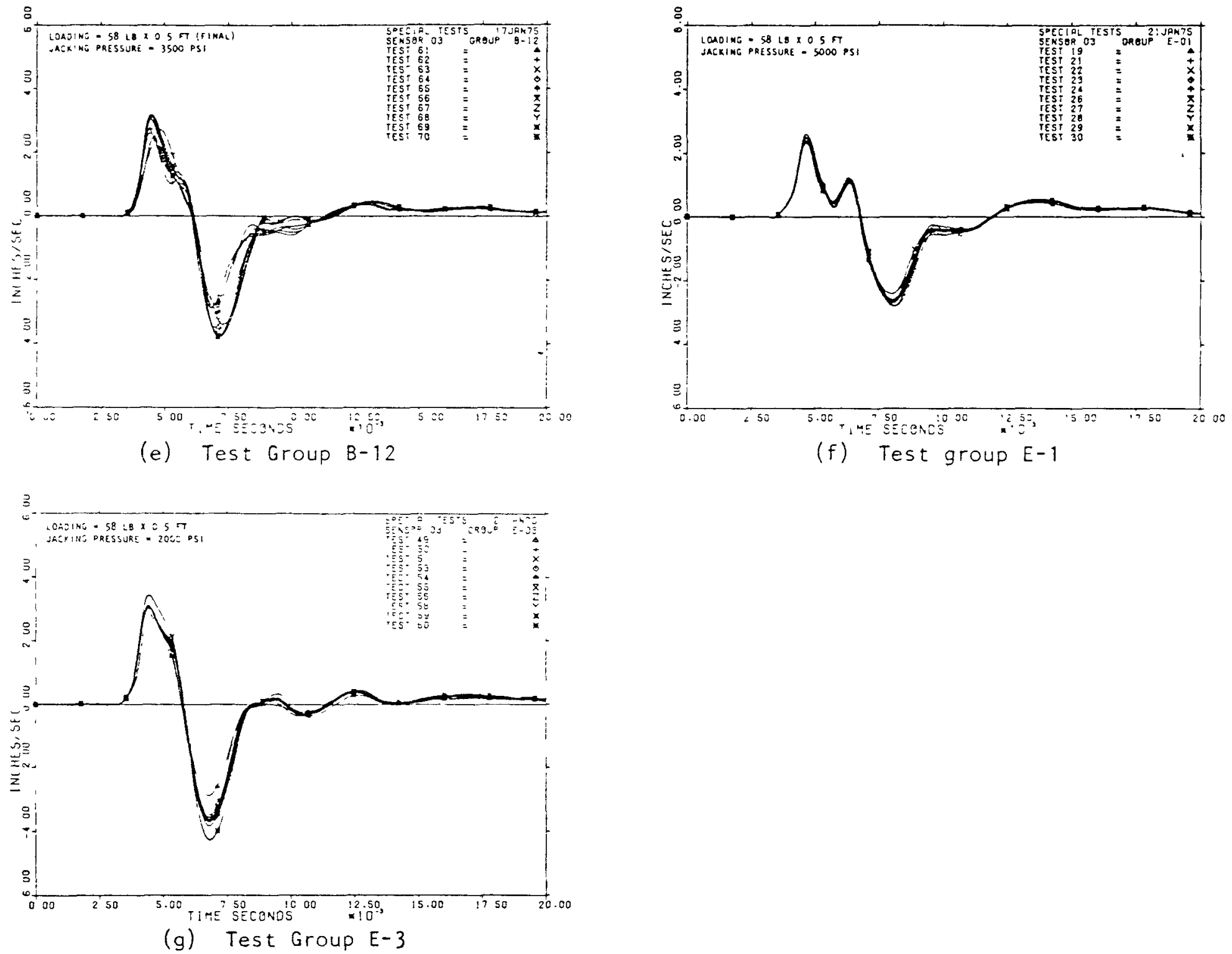

FIGURE F $-4 . \quad$ (CONCLUDED) 

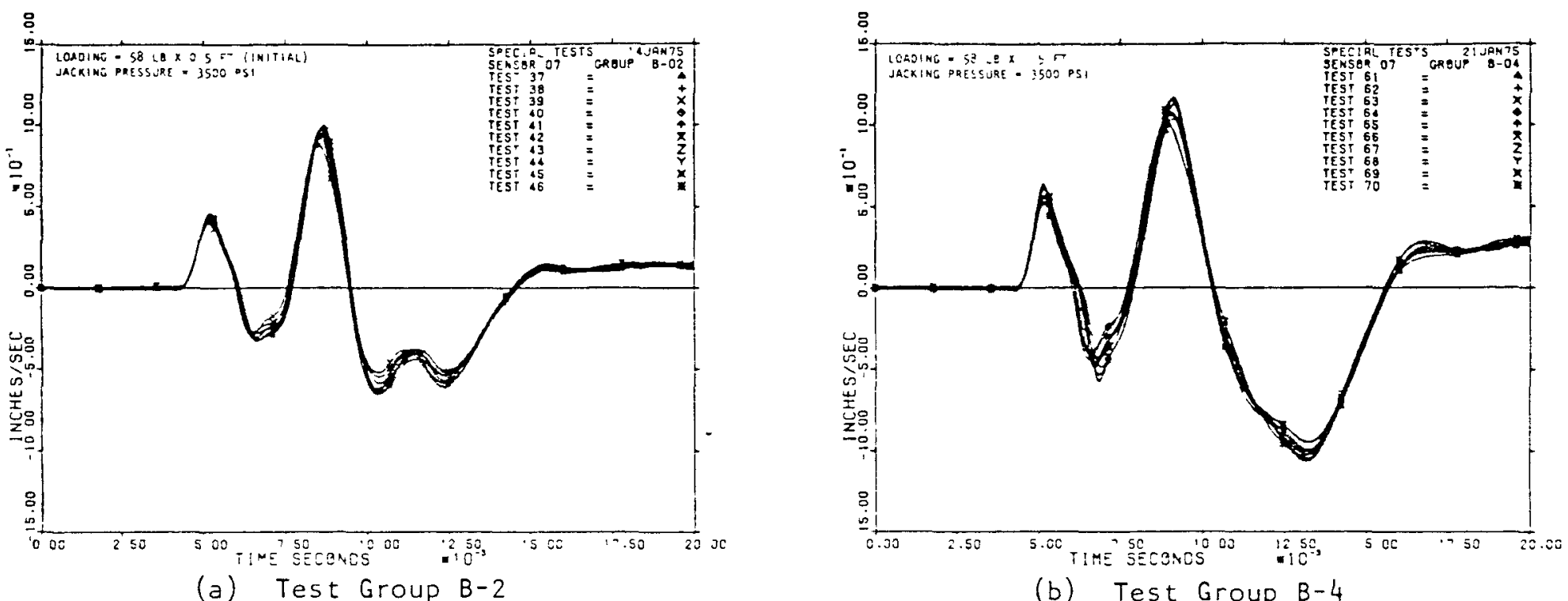

$\stackrel{\text { T1 }}{\stackrel{1}{*}}$
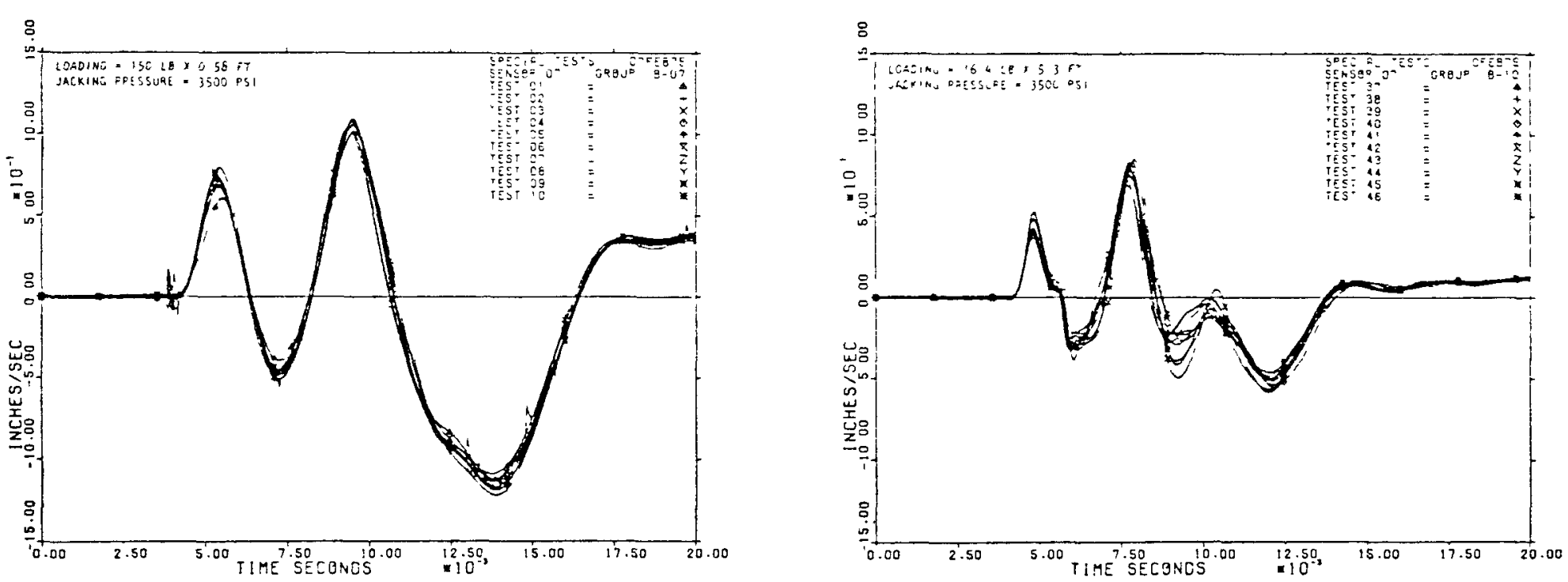

(c) Test Group $B-7$

(d) Test Group B-10

FIGURE F-5. REPEATABILITY OF VELOCITY HISTORIES AT SENSOR $7-24$ IN. FROM ANCHOR EDGE 

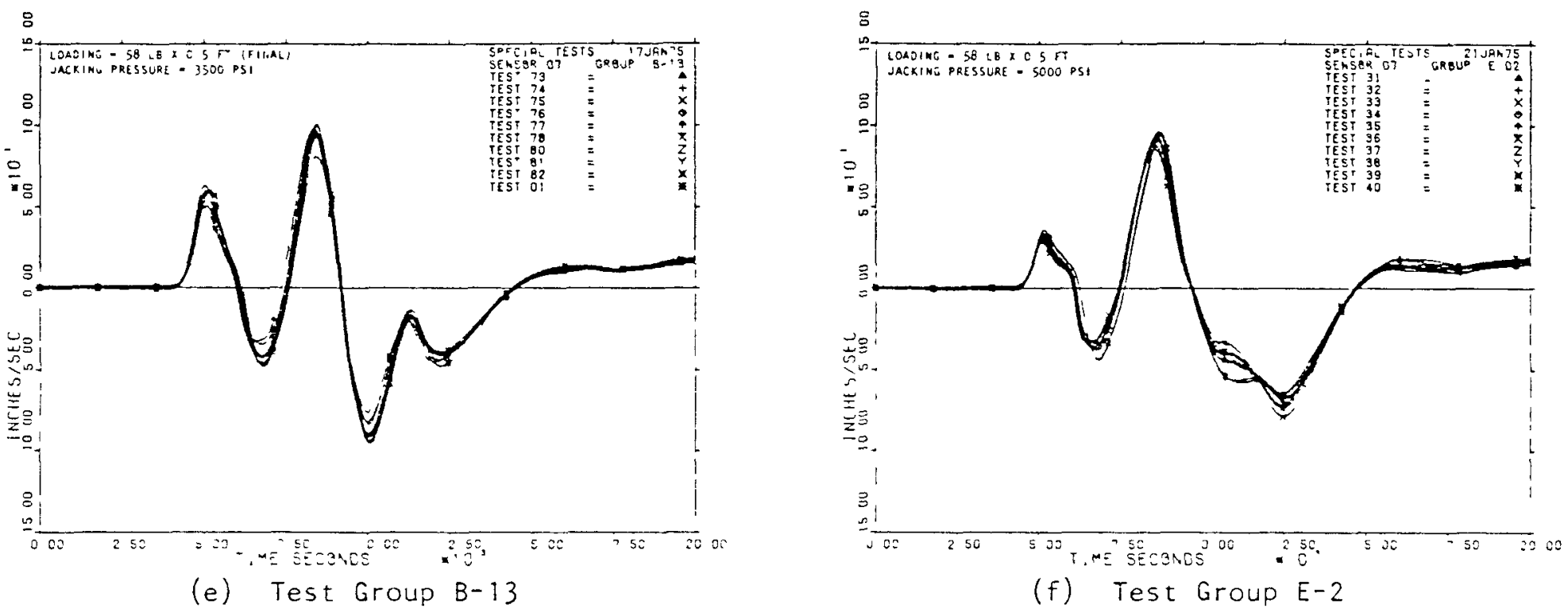

㛭

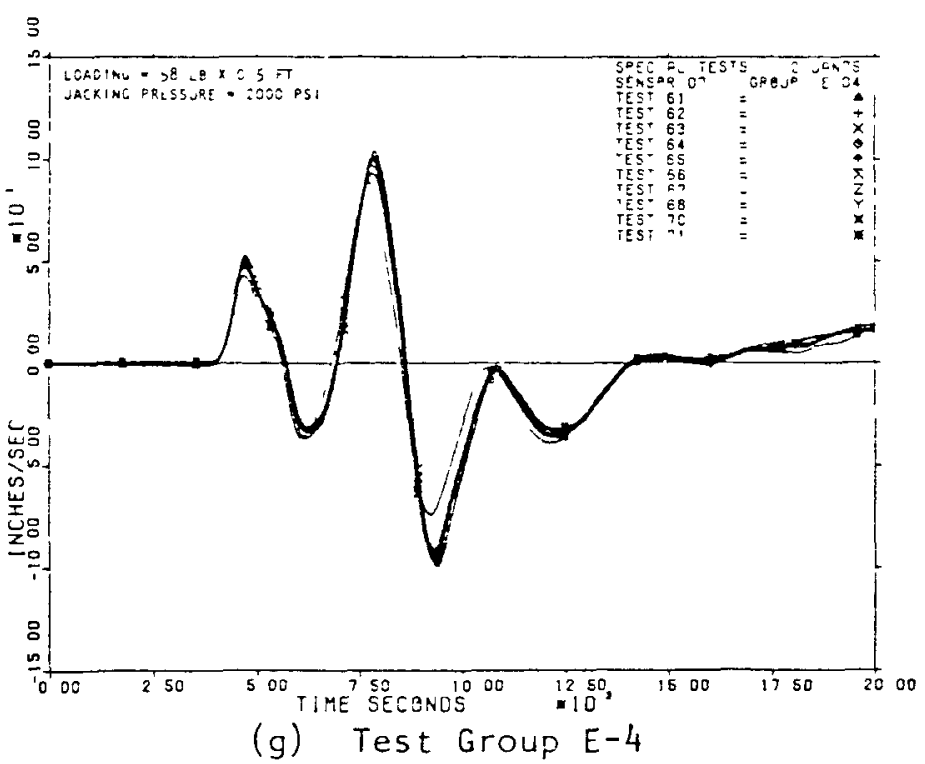

FIGURE F-5. (CONCLUDED) 


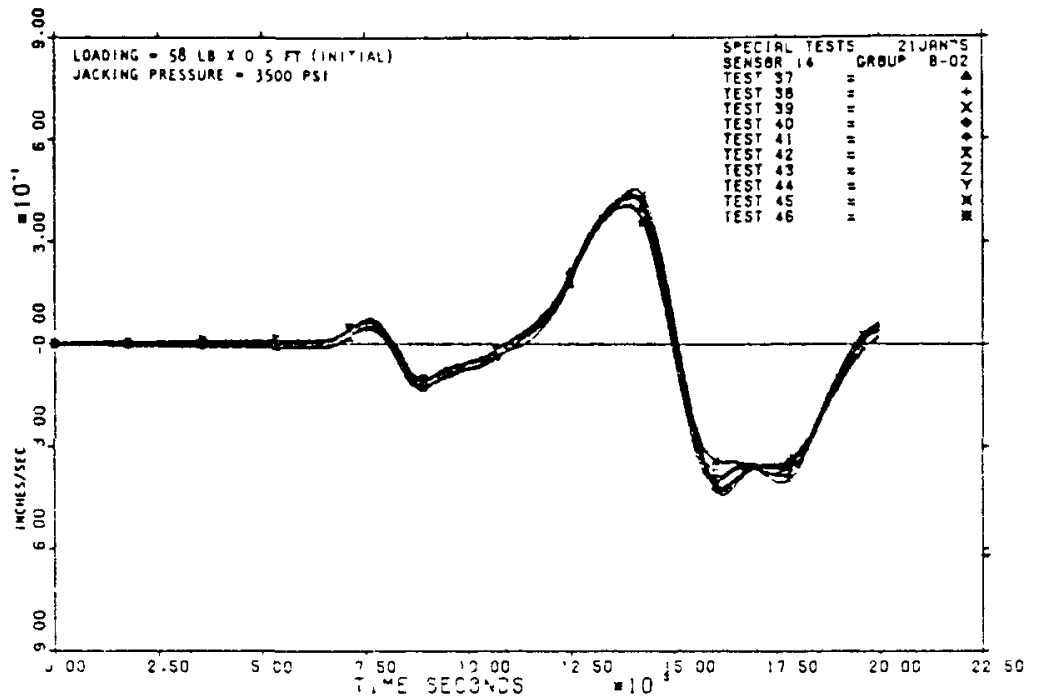

(a) Test Group B-2

卌

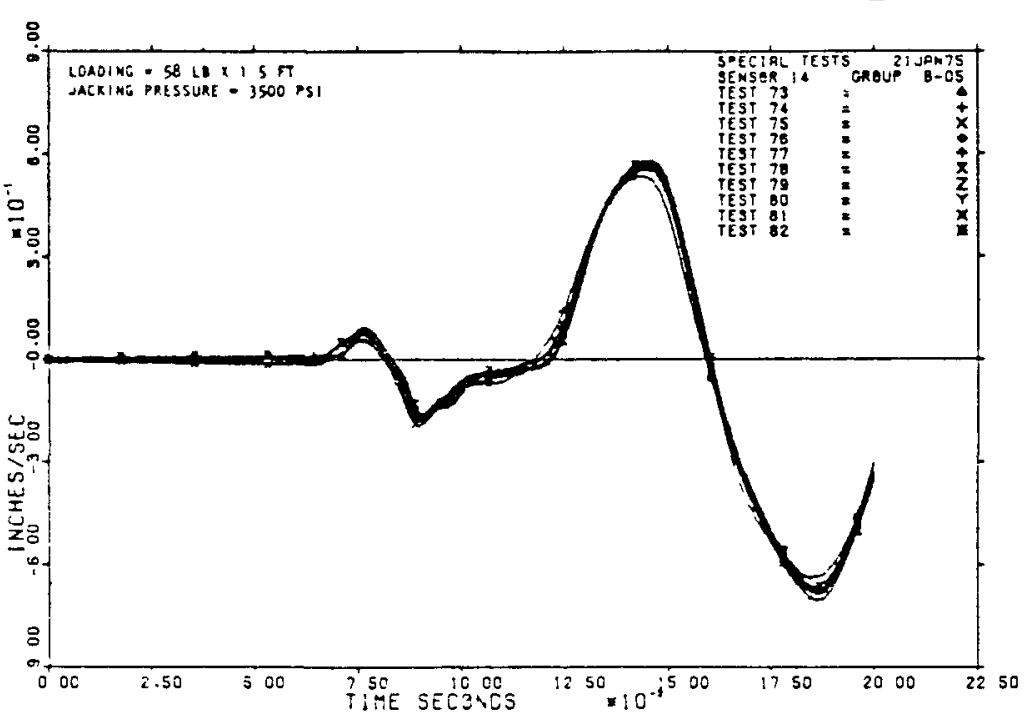

(b) Test Group B-5

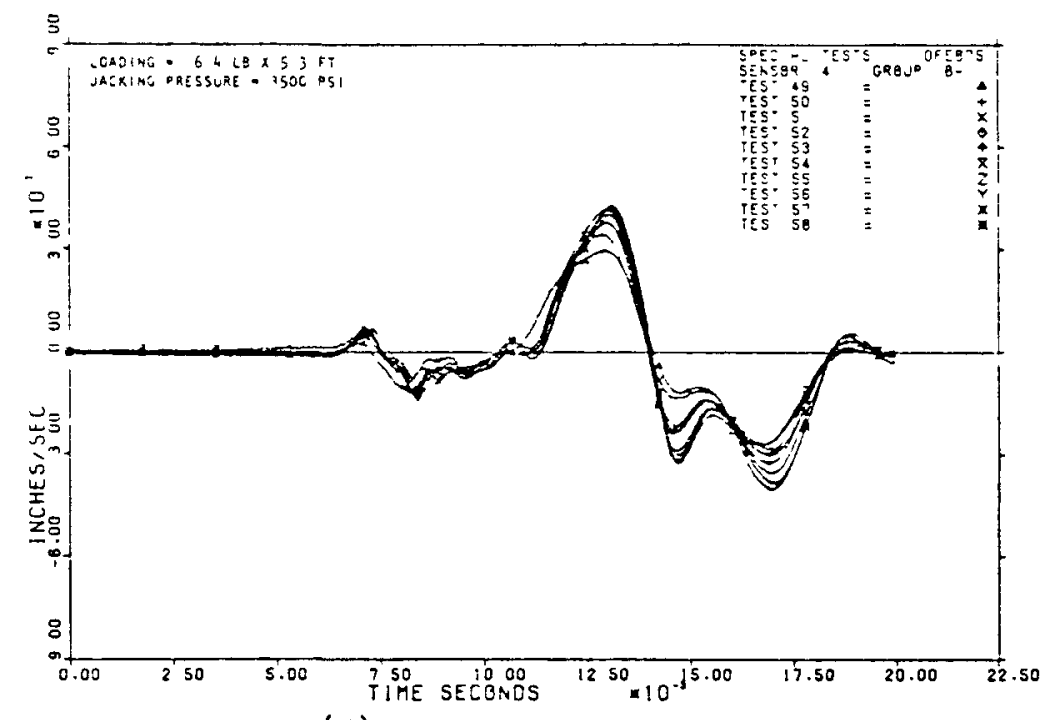

(d) Test Group B-11

FIGURE F-6. REPEATABILITY OF VELOCITY HISTORIES AT SENSOR $14-55$ IN. FROM ANCHOR EDGE 


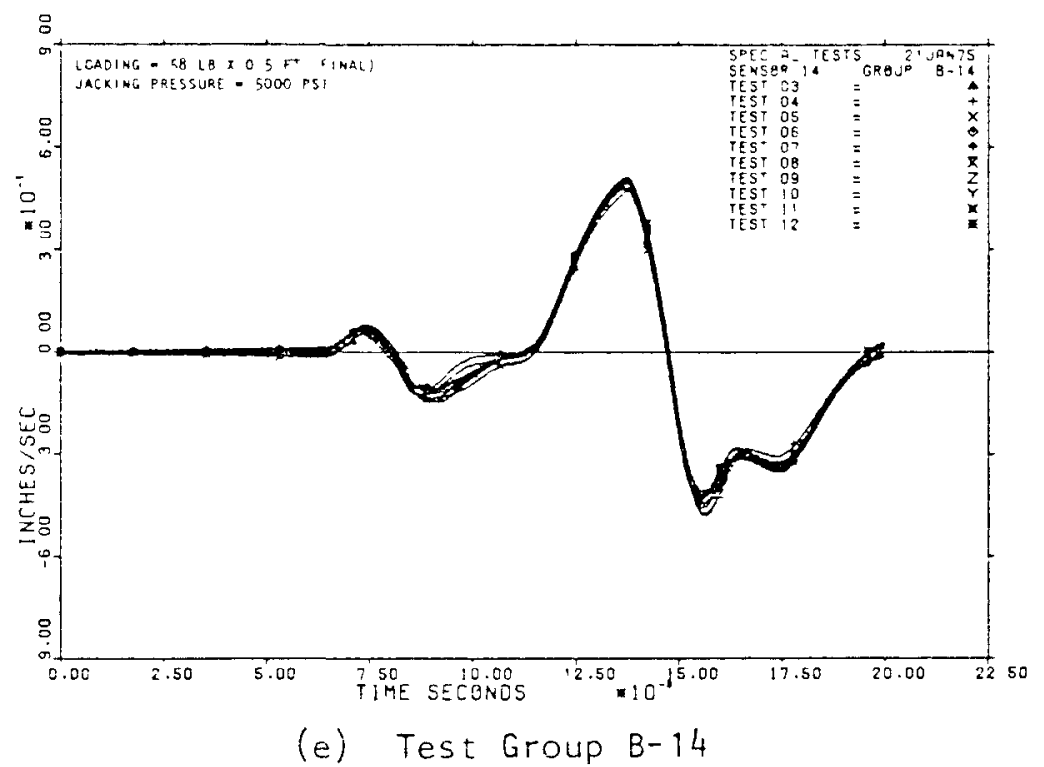

FIGURE F-6. (CONCLUDED) 
In this subsection, assessments of the effects of the position of the sensor are assessed. Two aspects of this problem have been investigated. The first deals with the spacing of a series of sensors along a line (which corresponds to axis $\mathrm{X}-\mathrm{X}$ in $\mathrm{Fig} . \mathrm{F}-1 \mathrm{~b})$. For the special tests, it was desired to place these sensors as closely as possible to one another in order to obtain the maximum refinement in the response measurements. However, if the sensor spacing was too small, adjacent sensors may have interacted with one another and could have distorted the resulting soil response measurements. Therefore, it was required to determine an optimum sensor spacing that avoids these "shadow" effects while still providing a sufficiently fine network of response measurements.

A second phase of this problem dealt with possible variations in the soil response measurements due to differences in the circumferential position of the sensors. This may be due to inhomogenities in the soil or non-uniform bearing of each of the anchor plates against the side of the borehole. An assessment of this problem was judged to be particularly important, since production tests have in the past typically utilized sensors placed at different circumferential positions, as shown in Fig. 1-1.

To investigate these potential effects, two different approaches were used. First, analytical solutions were used to define an optimum spacing for the special tests. These analyses are presented in Appendix $E$ and were used to guide the actual placement of the sensors along axis $X-X$ in Fig. F-Ib. As noted in this figure, these sensors were staggered so that they were at least three diameters apart, which is well within the guidelines indicated in Appendix E. 
A second approach for assessment of these effects utilized field tests in which velocity measurements were obtained at sensors located at near equal radial distances from the anchor but at different circumferential positions. An ensemble of these measurements, as generated from repeated applications of a 58-1b hammer and a 0.5-ft drop height were analyzed statistically to obtain mean and MSD velocity histories. Comparisons of these statistical results were used to indicate the presence of any non-symmetries in the circumferential distribution of the waves propagating from the anchor.

Results of these comparisons are shown in Fig. F-7. They indicate that the peak velocity amplitudes and corresponding wave shapes are generally similar regardless of the circumferential position of the sensor; however the rise times to peak velocity or the arrival times are not. In general, the sensors placed along axis $\mathrm{X}-\mathrm{X}$ (Fig. $\mathrm{F}-\mathrm{lb}$ ) tend to arrive later in time or have a longer rise time to peak velocity than do those sensors at the same radial position but offset circumferentially. rhis minor difference in arrival times was due, at least in part, to the distances of the sensor in the radial pattern being slightly closer to the center of the anchor than the corresponding sensors along the axis (see Table F-2). Except for this difference, the remainder of each velocity history does not seem to be substantially affected by the circumferential position of the sensor. It is noted that differences between the upper bound and lower bound MSD curves are quite small, indicating that the data is quite repeatable and that the above trends are not obscured by data scatter.

\section{F-3.3 Prior Load History Effects}

In the in situ soil testing approach, the soil layers at a particular deptin may be subjected to repeated load applications corresponding to different energy levels, in order to 

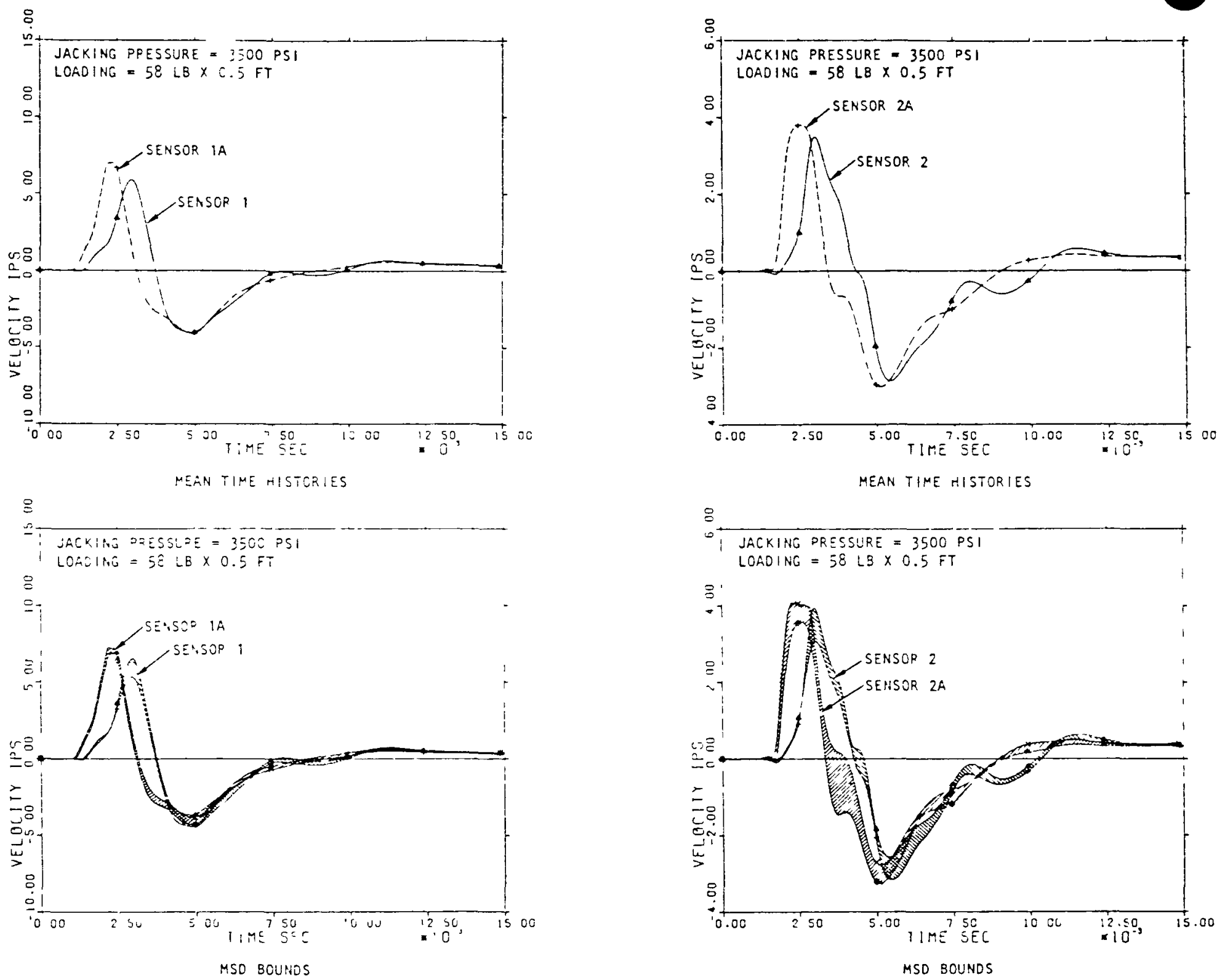

(a) Sensors located $3 \mathrm{in}$. from edge of anchor

(b) Sensors located $6 \mathrm{in}$. from edge of anchor

FIGURE F-7. STATISTICAL ASSESSMENT OF SYMMETRY AND SHADOW EFFECTS 

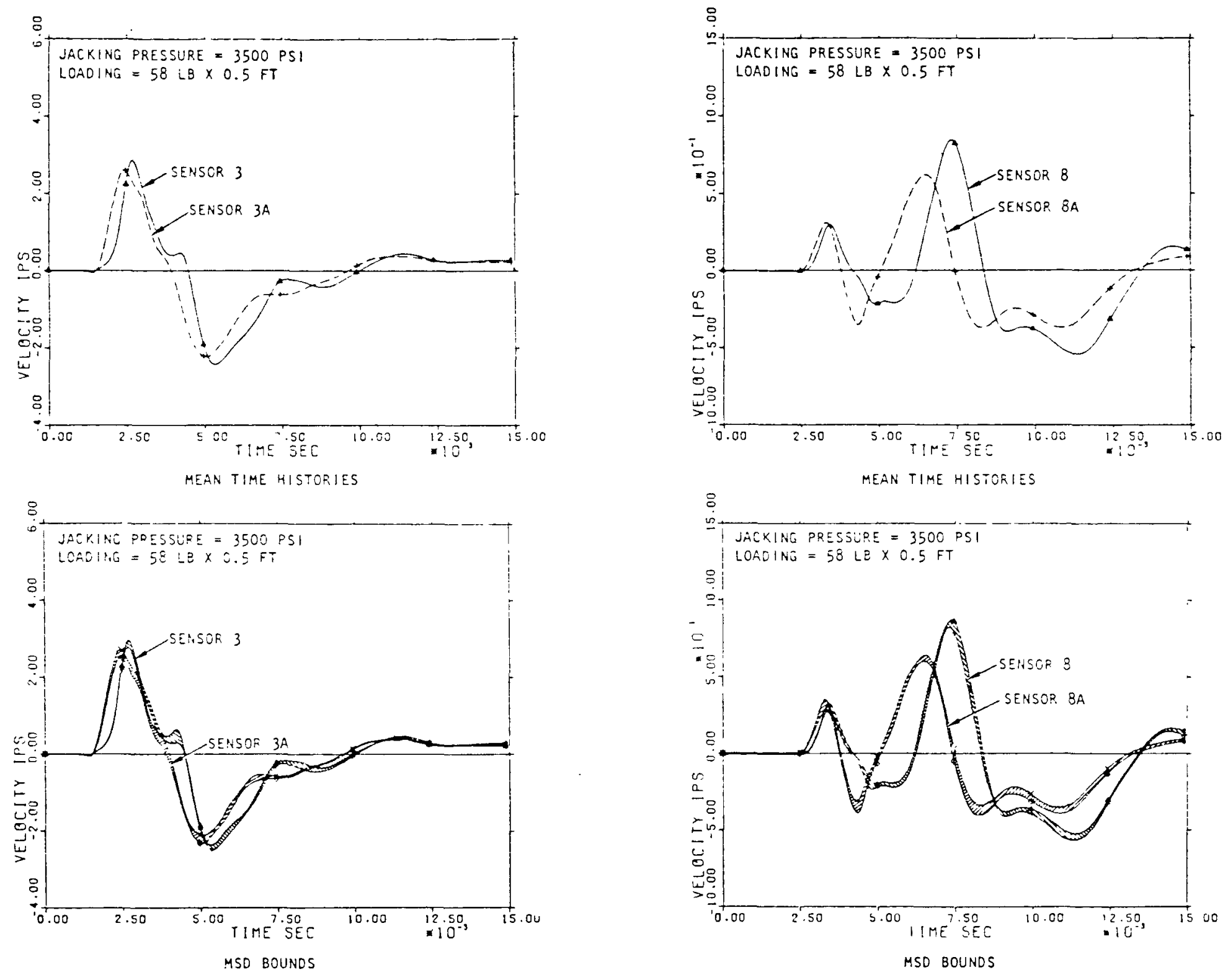

(c) Sensors located 9 in. from edge of anchor

(d) Sensors located $28 \mathrm{in.} \mathrm{from}$ edge of anchor 
obtain a wide spectrum of shear modulus vs. shear strain measurements. However, it is conceivable that for some soil materials, the measured responses during a given test may be affected by the number and magnitude of prior loadings to which the soil has been subjected. Therefore, it is important to investigate whether such prior load history effects have a significant influence on subsequent soil response measurements.

To investigate this effect, a series of initial tests were conducted during which a 58-1b hammer was dropped $0.5 \mathrm{ft}$ onto the Belleville springs. These correspond to Tests $A-1, A-2, B-1$, and $B-2$ in Table $F-1$. Then a large number of tests were conducted at higher energy levels (Tests B-3 to B-11), and these were followed by a series of tests corresponding to the initial loading conditions (Tests B-12 to B-14). To investigate prior load history effects, mean and MSD velocity histories corresponding to the initial and final sets of the $58-1 \mathrm{~b} \times 0.5-$ ft loadings were compared.

The above indicated comparisons at various sensors within the in situ soil test network are shown in Fig. F-8. They indicate that, regardless of the sensor location, prior load history effects are negligible. The mean velocity time histories may indicate some minor differences related to prior load history effects; however these differences are completely masked by the small amounts of data scatter observed in these test groups, as indicated by the comparisons of the MSD timehistory bounds.

\section{F-3.4 Variations in Hammer Weight and Drop Height for a Given Energy Level}

In the in situ soil test program, the input loads are typically specified in terms of an input energy level, determined as the product of the hammer weight and its height of free 

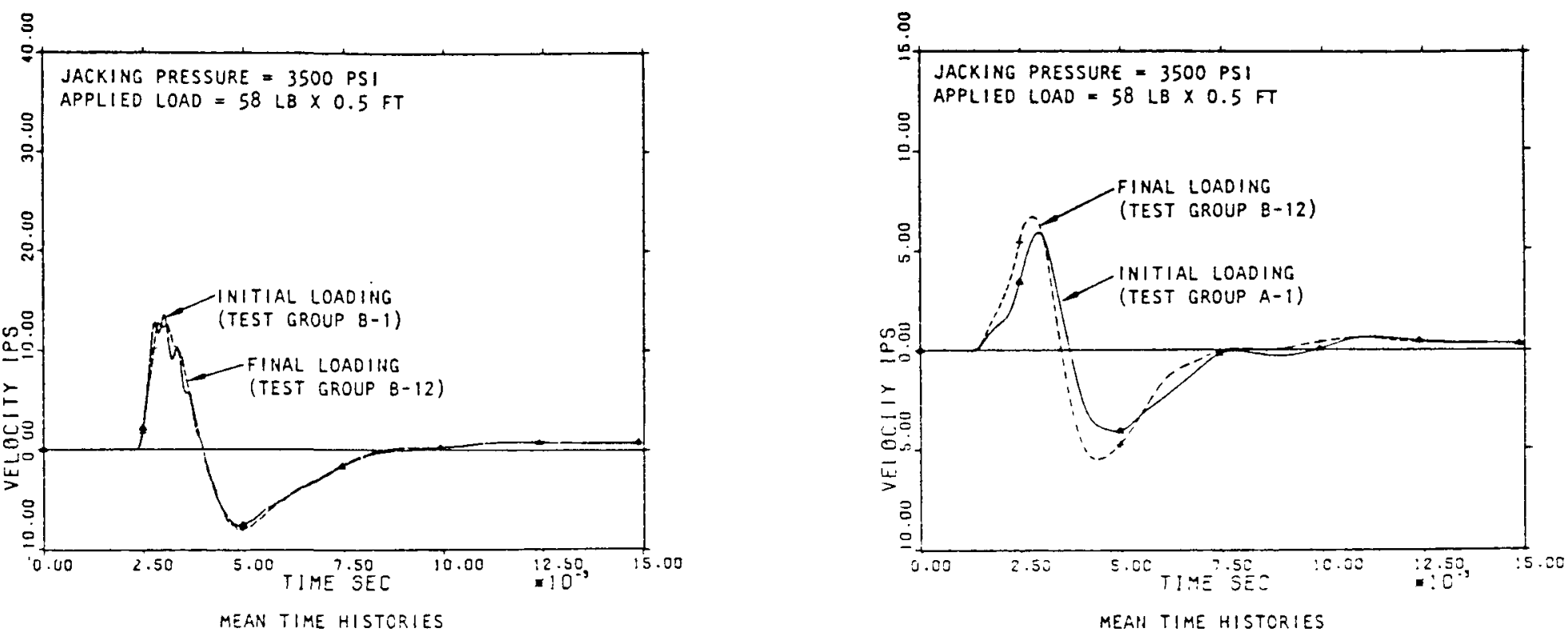

$\stackrel{1}{1}$
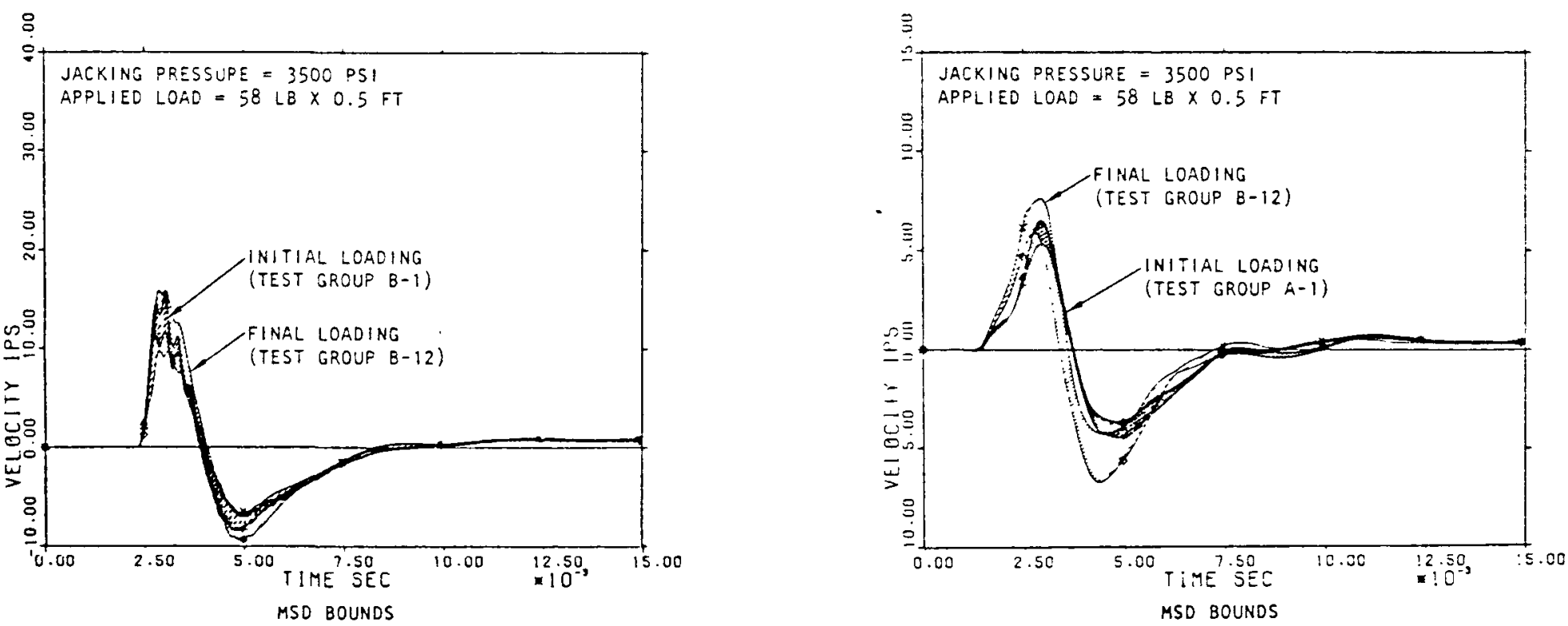

(a) Sensor 21 -- at anchor

(b) Sensor 1 -- 3 in. from anchor edge

FIGURE F-8. STATISTICAL ASSESSMENT OF PRIOR LOAD HISTORY EFFECTS 

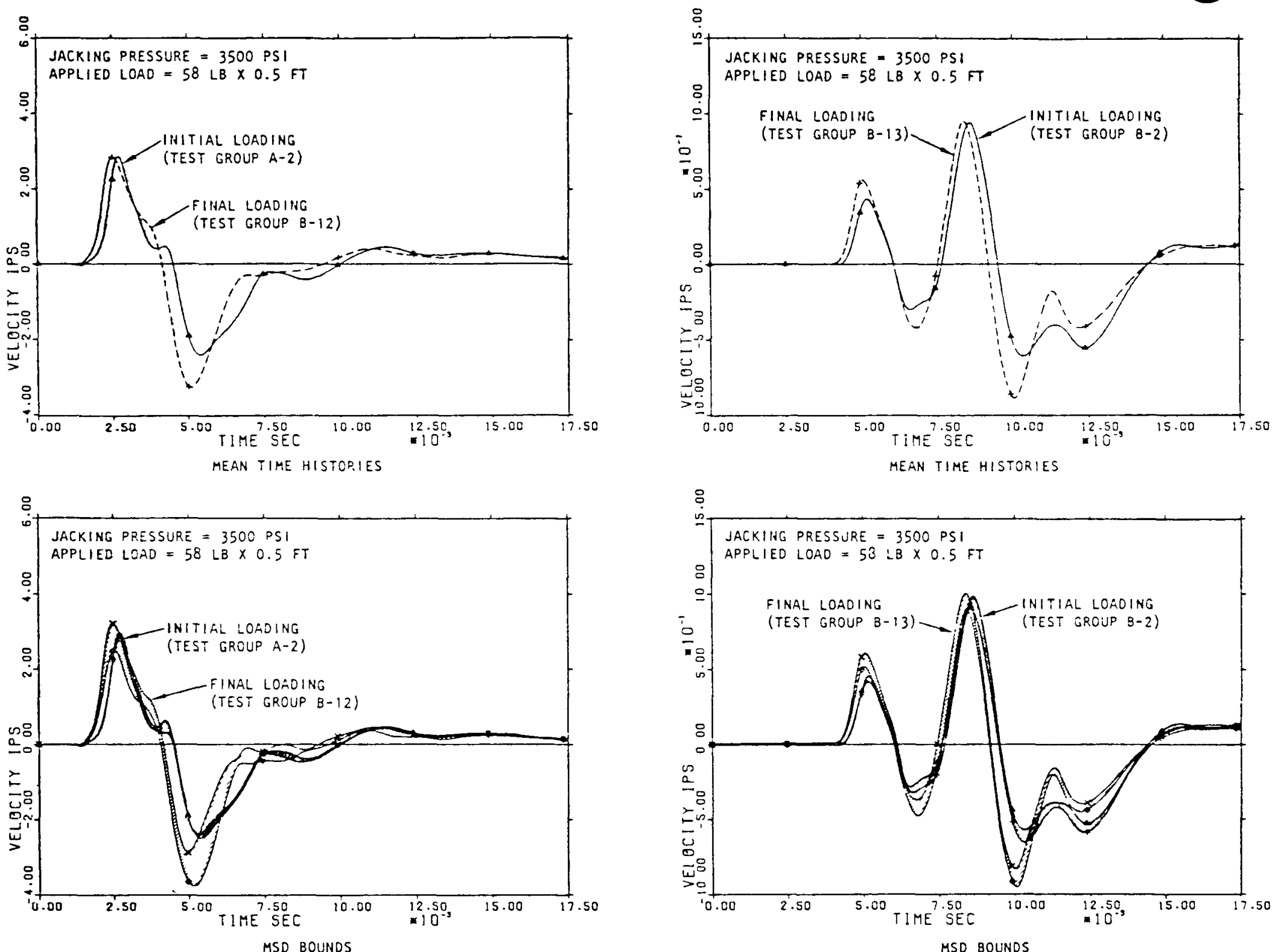

(c) Sensor $3--9$ in. from anchor edge

(d) Sensor 17 - 24 in. from anchor edge

FIGURE F-8. (CONTINUED) 

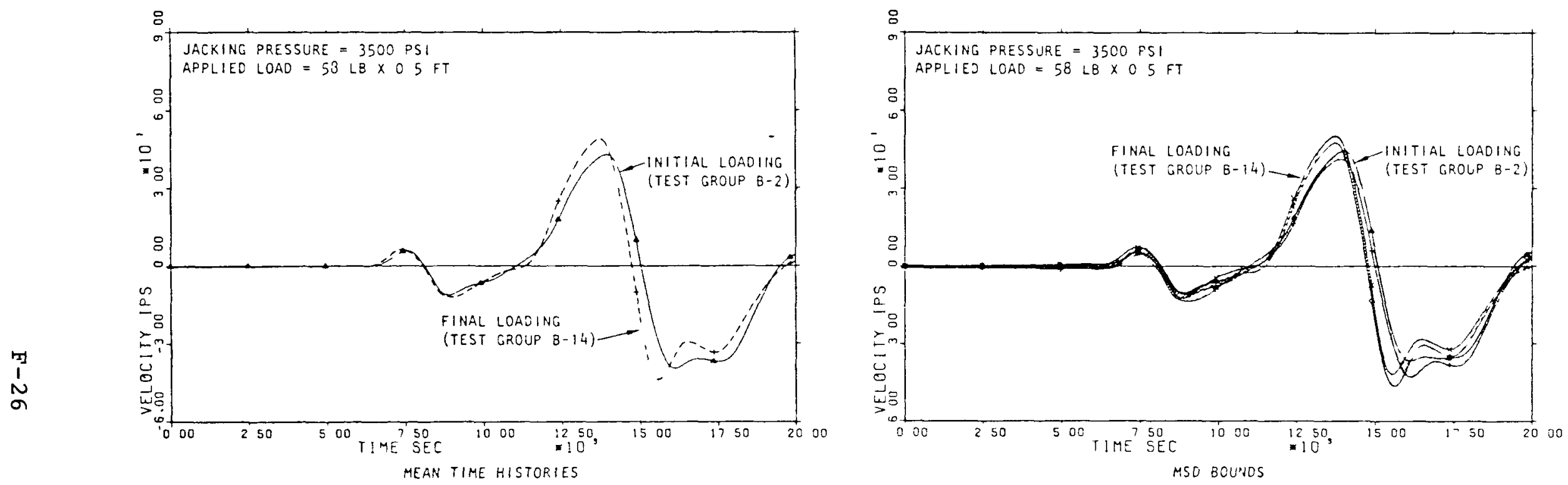

(e) Sensor 14 -- $55 \mathrm{in}$. from anchor edge

FIGURE F-8. (CONCLUDED) 
fall onto the Belleville springs. However, it is clear that a given energy level can be comprised of any combination of hammer weights and drop heights, and that each combination will represent a different dynamic system with different response characteristics.* Therefore it is important to investigate the degree to which these various combinations might affect the in situ soil response measurements and to gain some insight into those particular ranges of combinations that might be most advantageous for use in subsequent tests.

To investigate these effects as part of the special test program, three groups of tests were conducted. Each test consisted of a different hammer weight-drop height combination whose product corresponded to a total energy level of $87 \mathrm{ft}-\mathrm{lb}$. These three combinations are, respectively, $581 \mathrm{~b} x 1.5 \mathrm{ft}$, $1501 \mathrm{~b} \times 0.58 \mathrm{ft}$, and $16.41 \mathrm{~b} \times 5.3 \mathrm{ft}$. Soil response measurements corresponding to each of these combinations are compared in Fig. $\mathrm{F}-9$.

The results compared in Fig. F-9 show a number of interesting trends. First of all, as expected, the measured soil responses corresponding to each hammer weight-drop height combination are quite different, particularly in the immediate vicinity of the anchor. The largest peak velocity amplitudes tend to correspond to the 58-1b x 1.5-ft combination, while the smallest amplitudes generally occur when the 16.4-1b x 5.3ft combination was applied. These differences in the peak amplitudes were generally greatest at the anchor and tended to decrease with increasing distance from the anchor.

Changes in the weight of the hammer will affect the total mass of the coupled hammer-Belleville spring-anchor-soil system, while the variations in height of free fall of the hammer will influence its initial velocity as it first strikes the Belleville spring. 

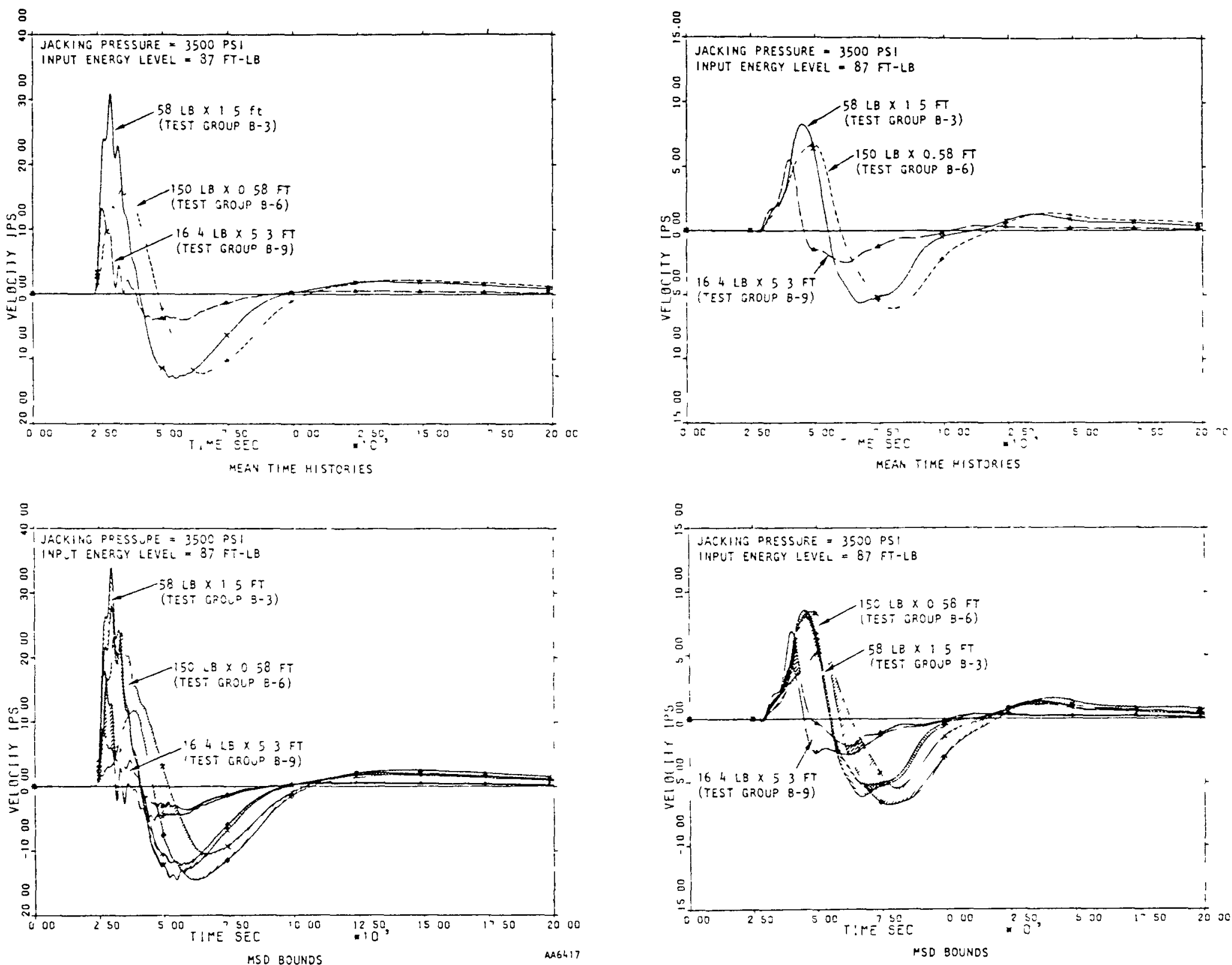

(a) Sensor 21 - at anchor

(b) Sensor 1 -- 3 in. From anchor edge

FIGURE F-9. STATISTICAL ASSESSMENT OF EFFECTS OF VARIOUS HAMMER WEIGHT-DROP HEIGHT COMBINATIONS FOR GIVEN ENERGY LEVEL 

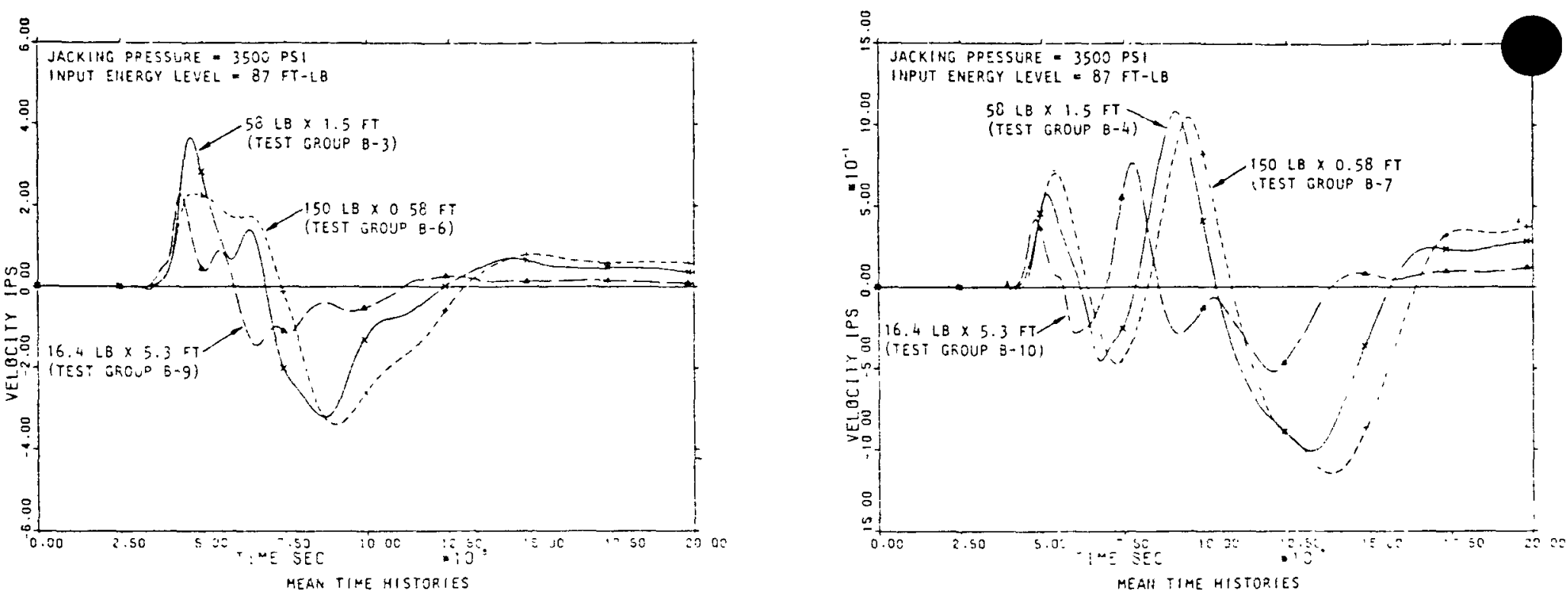

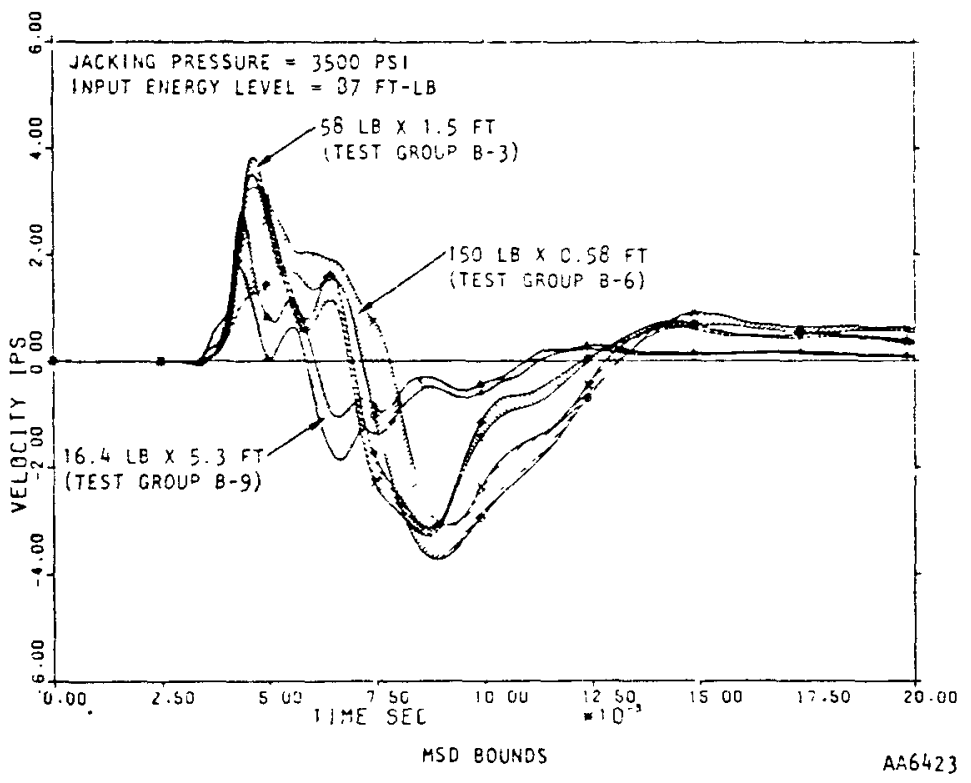

(c) Sensor 3 -- 9 in. from anchor edge

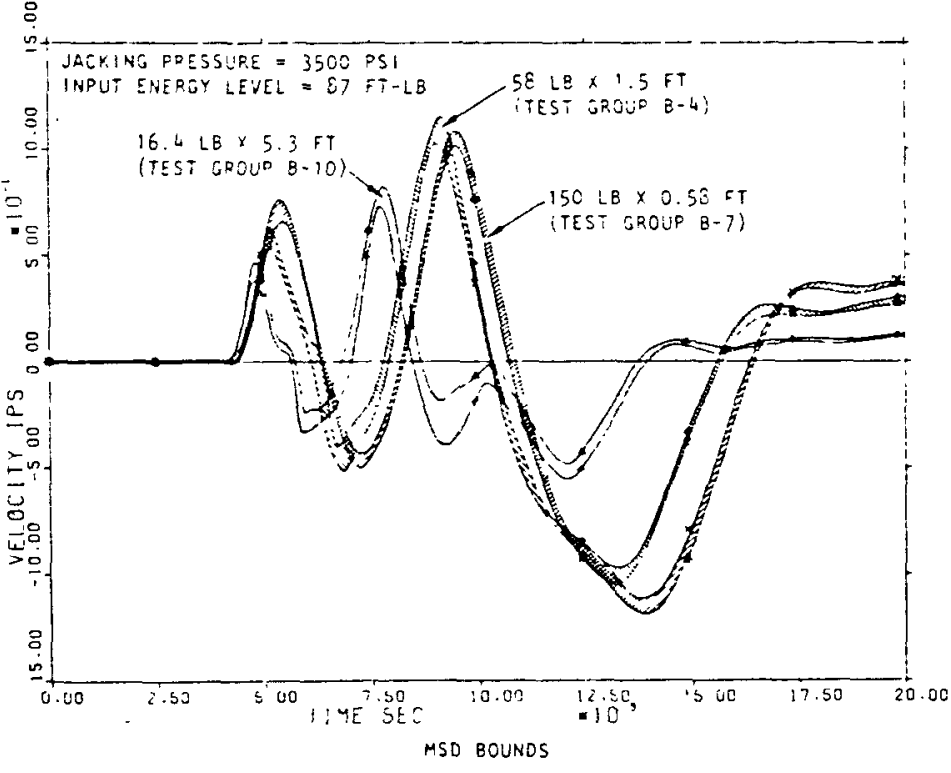

(d) Sensor 7 - 24 in. from anchor edge

FIGURE F-9. (CONTINUED) 

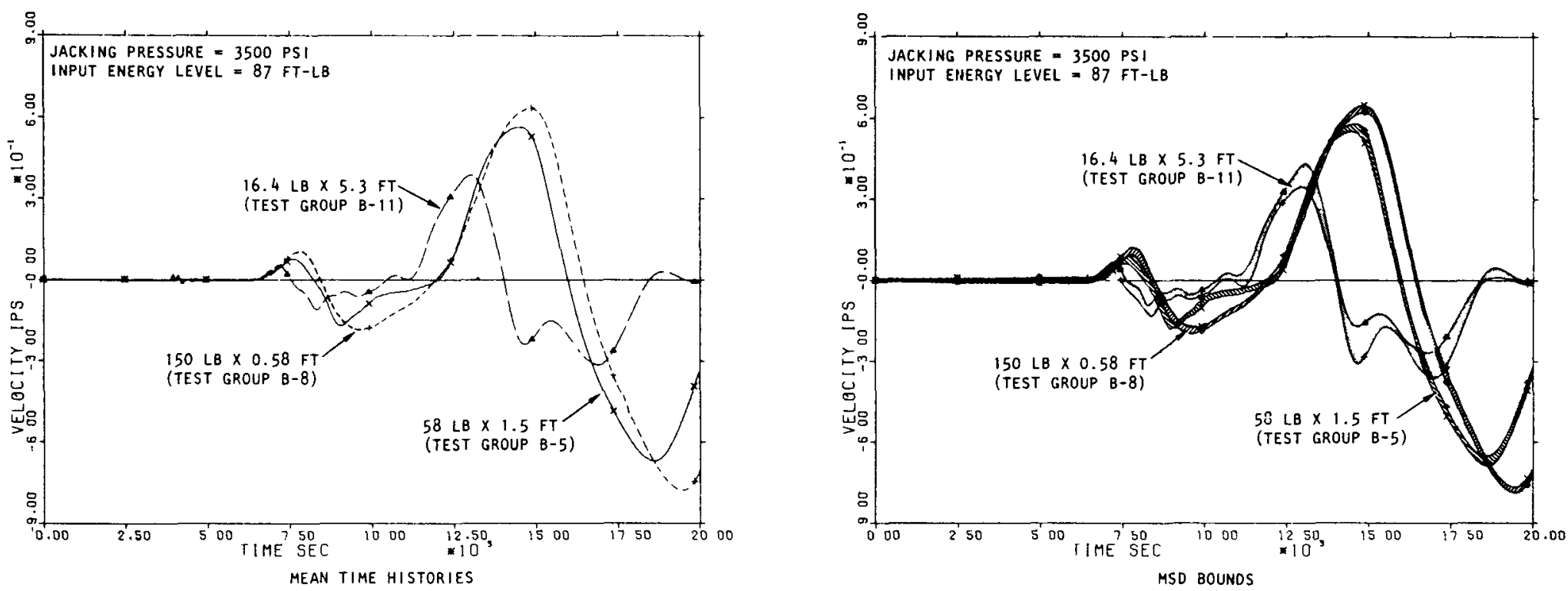

(e) Sensor 14 -- 55 in. from anchor edge

FIGURE F-9. (CONCLUDED) 
Another important trend relates to the width of the various velocity peaks measured during each set of tests. The widest peaks, which correspond to the lowest characteristic frequencies of the soil response, occur when the largest hammer weight, $150 \mathrm{lb}$, is applied. In contrast, the sharpest peaks, which correspond to the highest characteristic Erequencies, correspond to application of the smallest weight, $16.41 \mathrm{~b}$. This follows from the fact that the characteristic frequencies of the soil response are related to the inverse of the total mass of the system. An increase in the weight of the hammer obviously increases the total system mass, which in turn, will tend to widen the peak response peaks (i.e., reduce the characteristic response frequencies of the system).

A third trend relates to the scatter in the data. In the near-anchor regions, the substantial differences between the MSD time-history bounds from the 150-1b hammer tests indicates substantial scatter, while the widths corresponding to the smaller hammer weights are relatively narrow. However, at sensors located further from the anchor, these differences become smal1, indicating that the data from the 150-1b hammer tests are quite repeatable in this range. These differences in scatter over different regions of the soil may be attributed to the non-uniformities in the load applied by the heavy hammer, which might occur, for example, if the hammer is not flush when it first strikes the Belleville springs. Apparently, tests conducted using a heavier hammer are more prone to such nonuniformities than are tests with lighter hammers, possibly due to increased difficulty in handling the heavy hammer in the field. As the distance from the anchor increases, the soil response measurements become less sensitive to these nonuniformities.

As a final point, it is appropriate to compare the results from initial tests at a low energy level of $581 \mathrm{~b} \times 0.5$ 
ft (Fig. F-8) to the results indicated for the higher energy level (Fig. F-9). To carry out and interpret these comparisons, it must first be noted that the hammer weight influences the measured soil responses in two ways. First it affects the input energy level and dynamic loads to which the soil is subjected. Second, once the hammer makes contact with the Belleville springs, a coupled dynamic system is formed that consists of the hammer, springs, anchor, and soil. The weight of the hammer affects the inertial characteristics of this coupled system and thereby affects the manner in which this system responds to dynamic loads.

With this in mind, a comparison of the results from the high-energy and low-energy tests that correspond to the same hammer weight $(58 \mathrm{lb})$ can first be made. This comparison indicates that the 58-lb x 1.5-ft loading (Fig. F-9) results in soil velocity amplitudes that are consistently higher than the soil velocity amplitudes corresponding to the $58-1 \mathrm{~b} \times 0.5-\mathrm{ft}$ loading (Fig. F-8). These differences are as much as a factor of 2.5 at the anchor (e.g., a mean velocity of over 30 ips in Fig. F-9a vs. about 12 ips in Fig. F-8a) and range from about 25 to $35 \%$ at the various soil sensors. However, when the hammer weight used in the high-energy tests differs from that used in the low-energy tests, no such consistent trends can be observed. Figs. F-8 and F-9 indicate that, at a given sensor, the peak velocities from the high-energy tests using the 16.4-1b hammer and 150-1b hammer were not necessarily greater than those from the low-energy tests using the 58-1b hammer; in fact, at some sensors (e.g., Sensor 3) the low-energy tests produced higher peak velocity amplitudes than did the high-energy tests using the different hammer weights. These comparisons indicate that: 
a. An increase in the input energy level caused by changing the hammer weight will not necessarily result in increased soil response amplitudes. This is because the change in inertial characteristics of the coupled hammerspring-anchor soil system due to the changed hammer weight may in some cases be more important than the corresponding change in input energy level and may not tend to increase the soil response.

b. An increase in the input energy level caused by maintaining the same hammer weight and increasing the drop height will increase the soil response amplitudes. For this case, the inertial characteristics of the coupled system are unchanged; only the initial velocity of the hammer as it strikes the Belleville springs is increased because of its increased drop height.

\section{F-3.5 Jacking Pressures}

In order for loads to be transmitted from the anchor to the borehole, adequate coupling must be maintained along the anchor-soil interface. As described in Chapter 2, this coupling is developed by means of a vertically oriented hydraulic piston within the anchor. This piston, when expanded, forces a system of arms against the curved anchor bearing plates, forcing them outward and pressing them tightly against the sides of the borehole. A pump and gage located at the ground surface controls and maintains the hydraulic jacking pressures in the piston.

It is clear that the jacking pressures, applied as indicated above, represent a key component of the in situ soil test system. If these pressures are too low, slippage of the 
anchor will occur and adequate energy will not be transmitted to the soil medium. Alternatively, if the pressures are too high when compared to the dynamic stresses induced in the soil during the in situ test, they could affect the subsequent shear modulus measurements and, in the extreme, could induce shear failures in the soil surrounding the borehole.

The above discussion indicates the necessity of establishing a range of jacking pressures that will result in adequate coupling of the anchor to the soil, while not affecting the dynamic properties of the soil medium or inducing shear failures along the borehole. Therefore it is important to first define reasonable ranges of jacking pressures that can be applied by the hydraulic piston, and then to determine the extent to which the soil response measurements are affected by these ranges of pressures.

To carry out the statistical evaluation of this part of the special test program, three sets of tests were conducted, corresponding to radial jacking pressures of 2000 psi, 3500 psi, and 5000 psi. For each set of tests, repeated loadings corresponding to a 58-1b hammer dropped $0.5 \mathrm{ft}$ were applied, and soil velocity time histories were recorded during each application of these loadings. The resulting ensembles of time historles, measured at each sensor and corresponding to each jacking pressure level, were then analyzed statistically to develop composite time histories corresponding to a mean and to MSD bounds. These composite time histories at each sensor were then compared to indicate the sensitivity of the soil response to the assumed variations in jacking pressure.

Comparisons of the various composite time histories for the different jacking pressures and different sensors are shown in Fig. F-10. These comparisons indicate the following trends : 

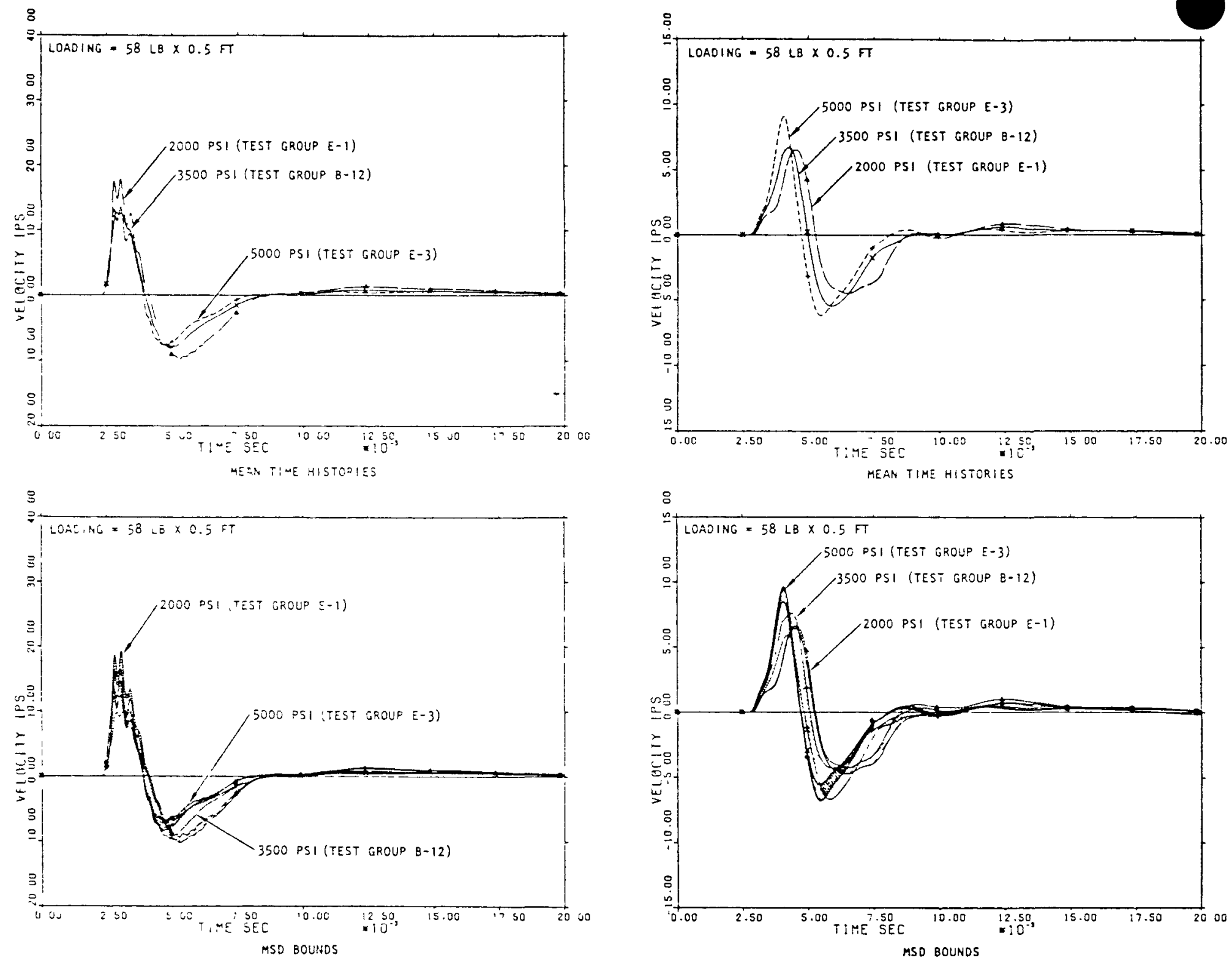

(a) Sensor $21:-$ at anchor

(b) Sensor 1 -- 3 in. from anchor edge FIGURE F-10. STATISTICAL ASSESSMENT OF EFFECTS OF JACKING PRESSURE 

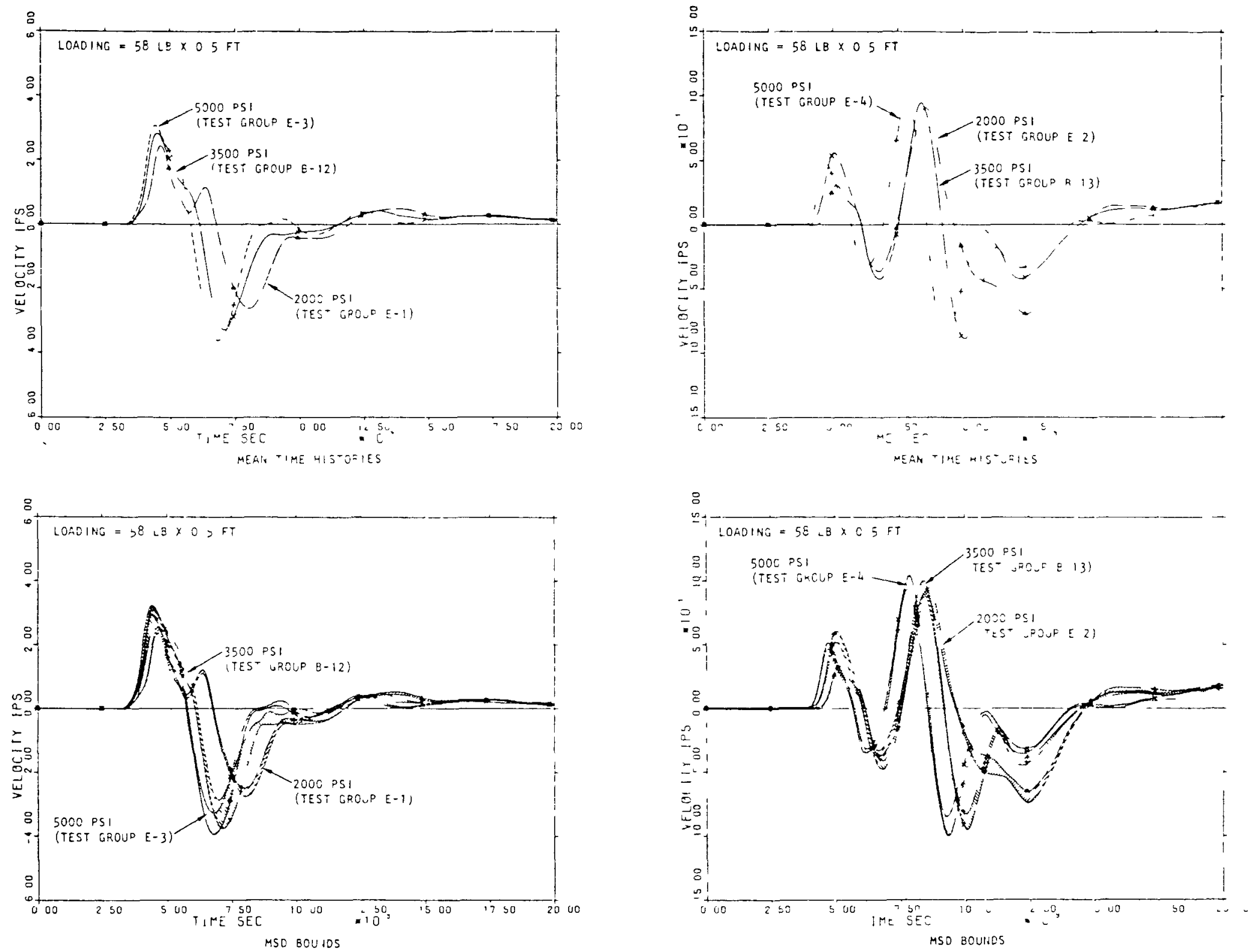

(c) Sensor 3 -- 9 in. from anchor edge

(d) Sensor 7 -- 24 in. from anchor edge FIGURE F-10. (CONCLUDED) 
a. At the anchor, the velocity histories corresponding to the 2000 psi level exhibit slightly larger peak velocity amplitudes; in contrast to this, the peak velocity amplitudes corresponding to this low jacking pressure that were recorded at sensors 1 and 3 exhibited somewhat lower peak velocity amplitudes. At sensor 7, differences in peak velocity amplitudes corresponding to the three jacking pressure levels are negligible. This suggests that greater slippage occurs along the anchor-soil interface during the tests conducted at the lower jacking pressure; however differences between the velocity amplitudes from the three jacking pressure levels were not substantial, suggesting that any slippage that might have occurred is small. Furthermore, effects of this slippage appear to be confined to a region only in the immediate vicinity of the anchor.

b. No significant trends could be observed regarding differences in velocity histories due to changes in jacking pressure of from 3500 psi to 5000 psi. Only slight, highly localized differences between the velocity histories corresponding to these two jacking pressures could be observed (see Fig. F-10b corresponding to the Sensor 1 measurements), but these are not significant in view of the negligible differences between the measurements at the other sensors.

The above trends suggest that for the input loading conditions, soil properties, and ranges of jacking pressure considered in these special tests, the variations in jacking pressure have a negligible effect on the soil response measurements. The potential effects of jacking pressure should be kept in mind when using the in situ soil test at other soil conditions and possibly for larger loads; however the special test results suggest trat, for reasonable ranges of jacking pressures and input loads, the effects of jacking pressure on 
the in situ dynamic soil properties will be confined to a highly localized region around the anchor. Reasonable jack pressures should not affect the overall free-field soil response measurements to any substantial degree as long as slippage is not excessive producing, in effect, distorted wave shapes.

\section{F-4 SUMMARY AND CONCLUSIONS}

This appendix contains results of a statistical assessment of results obtained during a comprehensive special test program, whose purpose was to investigate the effects of certain key test parameters on the in situ soil response measurements. The parameters investigated correspond to repeatability of the data, symmetry and shadow effects, prior load history effects, the effects of variations in hammer weight and drop height for a given input energy level, and jacking pressure effects.

The use of statistical assessments of an ensemble of test results, as described in this appendix, provides a meaningful basis for evaluating trends that arise from variations in the test parameters. They offer one advantage over nonstatistical studies based on a limited number of tests, since they indicate to what extent scatter in the data may influence apparent trends that are observed. Since any number of systematic or random effects can lead to data scatter in a complex testing approach of this type, it is important that this scatter be considered in this manner. If, as shown, the statistical data indicates a high level of repeatability and small scatter of data, nonstatistical studies can be conducted also to evaluate these same effects in greater detail, as well as other effects not included herein. The results of these additional studies are presented in the next appendix.

Results of this statistical assessment have indicated certain clear-cut trends regarding the effects of the various 
test parameters on the soil response measurements. Of course, judgement must be exercised when extrapolating these particular trends to other site conditions and other load applications; nevertheless, the results contained in this appendix serve to increase our understanding of the overall in situ soil testing approach.

The particular trends observed from this statistical assessment of the special test data are as follows:

a. The measured velocity histories are generally quite repeatable, as evidenced by the close correlations between the overlaid time histories and the relatively narrow widths of the composite velocity histories corresponding to MSD bounds. The only lack of repeatability occurred at near-anchor soil response measurements when an extremely heavy hammer (150 1b) was used; this suggests that, in future in situ soil test applications, hammers of a more moderate weight should be used to avoid this potential source of data scatter.

b. Except for minor differences in rise times to peak velocity caused at least in part by minor differences in sensor distances from the anchor, symmetry and shadow effects did not appear to influence the soil response measurements to any substantial degree. It is noted, however, that in these studies, sensors located along axis X-X in Fig. F-lb, were deliberately staggered so that their clear spacing was within guidelines set by prior analytical solutions for avoiding shadow effects (Appendix E).

c. For the conditions considered in these special tests, prior load history effects were shown to have a negligible effect on the soil response measurements. 
d. The particular hammer weight and drop height used to represent a given input energy level could have an important effect on the resulting soil response measurements, particularly near the anchor. An increase in the input energy level, by changing the hammer weight and drop height relative to prior tests conducted at lower energy levels, will not always result in increased soil response amplitudes relative to these low energy tests. Therefore, it is important to utilize the particular hammer weight-drop height combination that maximizes the near-anchor soil response for a given energy level; trial-anderror field tests or analytical solutions may be required to establish these combinations.

e. The range of jacking pressures considered in these special tests resulted in only highly localized effects on the near-anchor soil responses and did not affect the overall soil response characteristics to any noticeable degree. 
APPENDIX G

GENERAL ASSESSMENT OF SPECIAL TEST RESULTS 


\section{APPENDIX G}

GENERAL ASSESSMENT OF SPECIAL TEST RESULTS

When a test procedure is being studied with experimental testing, there are always minor adjustments or measurements that can be readily incorporated or accomplished which can provide greater insight into a number of test conditions or parameters. A number of these parameters were evaluated statistically in the previous appendix. From these studies, it was generally shown that from repeated tests, there is a high level of data repeatability and that with few exceptions the scatter of data is generally small. As a result, it was considered desirable to study some of this same data together with supplemental test data using a non-statistical approach. This approach was necessary because these supplemental tests were not repeated a large number of times and were originally obtained under the assumption that the data, as indicated in the previous appendix, is repeatable. Rather, these tests are fewer, but cover a wider range of test variables. In particular, those parameters or conditions studied in this appendix include:

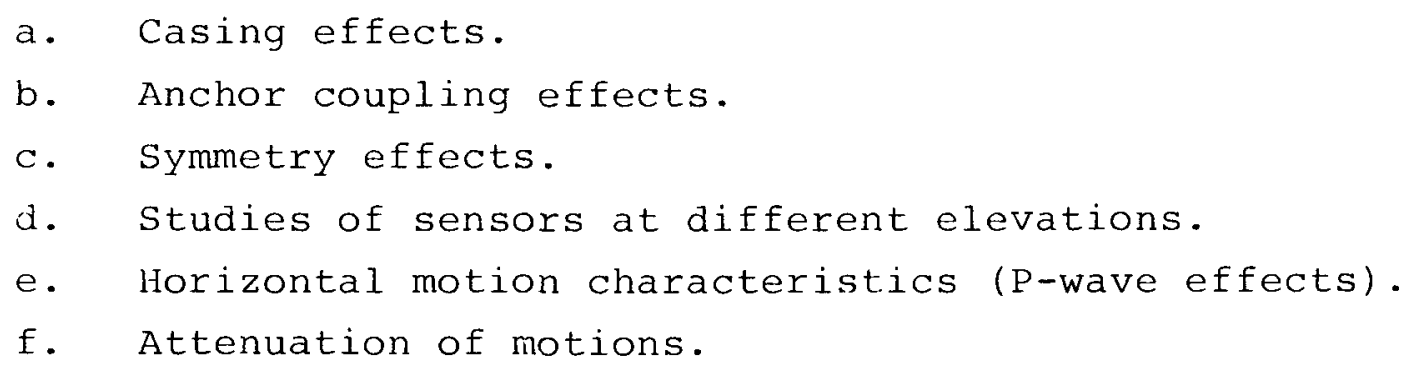

Most of these results are based on a direct review of the data followed by a semi-empirical evaluation of the results.

\section{G-1 CASILJ EFFECTS}

One objective was to determine if casing could be used with the production test primarily to retain soils highly subject 
to caving. For this program, four types of casing were systematically hand placed within the backfill material along with adjacent free field sensors. These casings consisted of 3-inch ABS plastic casing, 3-inch aluminum casing, 3-inch corrugated (vac-u-flex) hose, and 3-inch standard steel casing. Two groups consisting of two casings and a free field sensor as shown in the plan, Fig. 5-1, were positioned together each about 45 inches from the closest part of the anchor. This distance was considered representative of that distance between the anchor and the first sensor location in the conventional test. Then sensors were lowered inside each casing and coupled at the depth (elevation -5 feet) immediately adjacent to each free field sensor. Simultaneous measurements from sensors in the anchor, the free field, and each of the two adjacent casings were then made. Comparisons of corresponding signal shapes, amplitudes and times of arrival could then be accomplished, studied and evaluated.

From these tests the following results were obtained:

a) Average variation of particle velocity amplitudes with free field measurements:

Plastic Casing Aluminum Casing Corrugated Casing Steel Casing
Amplitude of signal in casing was $7 \%$ low Amplitude of signal in casing was 35\% low Amplitude of signal in casing was $26 \%$ high Amplitude of signal in casing was $29 \%$ low

b) Time lag between free field sensors and sensors in casing :

Plastic Casing

Aluminum Casing Corrugated Casing Steel Casing
Signal arrived $.46 \mathrm{msec}$ later in casing Signal arrived $.68 \mathrm{msec}$ later in casing Signal arrived $.03 \mathrm{msec}$ later in casing Signal arrived .73-1.77 msec later in casing 
'i'hese data in general reflect the logical conclusion that the more resistant or stiffer the casing to vertical motion, the smaller is the resulting amplitude of the measured signal and the greater is the lag in time from the free field condition.

In addition to these results, the shape of the primary velocity signal recorded in the casings was only slightly altered from the signal recorded by the free field sensors. As a result of this and the above results, a valid conclusion would be that any of the four casings could be used in sensor holes provided adjustments in time and amplitude are accomplished, when applying the data with sensors on the anchor. When considering all of the above factors, the plastic or the corrugated casing appears to be the best of these casings for practical use with this test. of these two, the plastic casing is probably the better primarily because it is far easier to handle and install. The steel casing produced the largest variation in time. Since time is a very sensitive parameter, much more so than amplitude, these large variations or lags in time are not desirable and therefore the steel casing is the least desirable of the four casings.

Based on the above data, the following time and amplitude adjustments are recommended for use with the in situ test:

\begin{tabular}{lcc} 
Casing & $\begin{array}{c}\text { Time } \\
\text { (msec) }\end{array}$ & $\begin{array}{c}\text { Amplitude } \\
\text { o }\end{array}$ \\
\cline { 2 - 3 } Plastic & .4 & 0 \\
Aluminum & .6 & 35 \\
Corrugated & 0 & -25 \\
Steel & 1.2 & 30
\end{tabular}

The adjustment in time is only made for the sensors within casing (i.e., the Equivalent Free Field Time = Measured Time in casing less the above adjustment). 
To study the importance of anchor coupling effects in the near-anchor region, a number of tests were conducted to obtain measurements at or near the anchor under different jack pressures. By comparing force and response characteristics of the anchor and the response of the first "close-in" adjacent sensor (Sensor No. 1) as a function of jack pressure, the importance or influence of this parameter on the near-anchor responses can be shown.

To study this effect in relation to the forcing characteristics applied to the anchor, the jack pressure is first compared in Fig. G-l with the load measurements obtained at the top of the anchor with the load cell. Significant measurements from the typical triangular or parabolic load pulse are the peak load value, $F_{1}$, the total load pulse auration time, $T_{d}$ ' and the rise time of the pulse, $\mathrm{T}_{r}$. For this comparison, the drop heigit and mass are held constant while the jack pressure is varied over five different values.

In the upper plot, the applied load first increases as the jack pressure increases, reaching a maximum for $\sigma_{j}=3,500$ psi, and then decreases for higher jack pressures. These results would indicate that the anchor-soil system becomes more rigid as the radial stress applied to the soil increases within the Iimits of the elastic state. This fact is to be expected, since a higher radial stress in general increases the resistance to slippage. However, excessive jack pressures cause higher radial stresses which combined with the stresses generated from the impact may exceed the bearing resistance of the soil, thus explaining the drop in the applied load at higher jack pressures. The rise and the duration times of the load pulses follow the same trend as the maximum load. The total impulse defined as the area of the load-time curve also has a maximum value for 


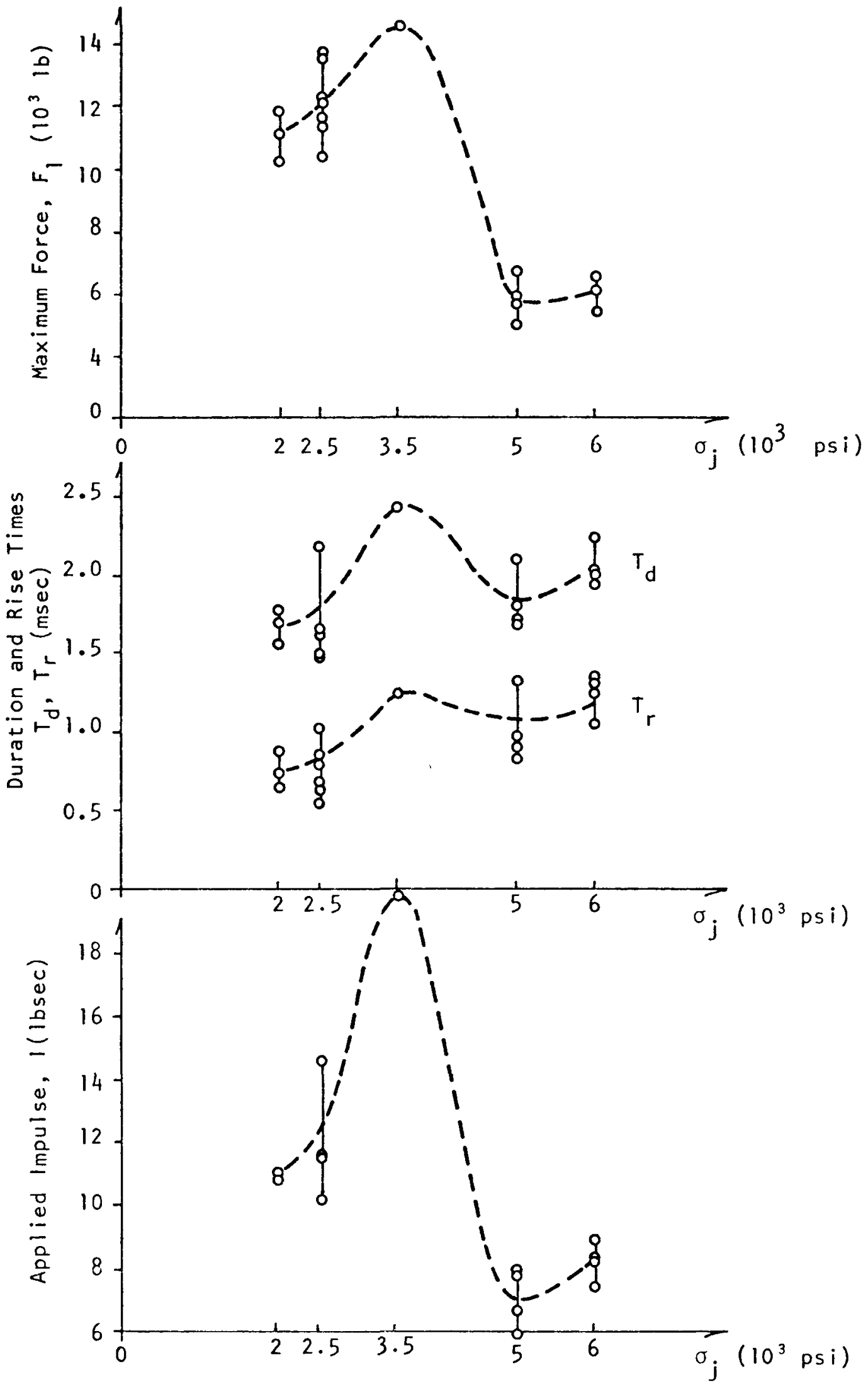

FIG. G-1 EFFECT OF JACK PFESSURE ON APPLIED LOAU FOR COISTAIT ENERGY. $W=58 \times 0.5 \mathrm{lb} \mathrm{ft}$ 
$\sigma_{j}=3,500$ psi.

The skewing constant $0=\mathrm{T}_{\mathrm{r}} / \mathrm{T}_{\mathrm{d}}$ of the load pulse has an average value of .51 for these tests with a tendency to increase from . 4 for smaller $t_{1} .6$ for larger jack pressures. These values indicate tnat the load pulse is symmetrical for the intermediate pressure of $3,500 \mathrm{psi}$, it has a steeper rise time for smaller pressures and it decays faster for larger pressures. For the tests performed at the constant jack pressure of 3,500 psi the average $\sigma$ is .48 .

The response characteristics of the anchor to an impact also depend on the degree of coupling with the soil mass. ilg. G-2 shows the effects of increasing the jack pressure, irom 2,000 psi to 6,000 psi, on the velocity and displacement of the anchor, and sensor No. 1 under a constant hammer weight and height of drop. It is observed that the velocity and the displacement at the anchor decrease to minimum values for $\sigma_{j}=$ 3,500 psi and then slightly increase for the higher $\sigma_{j}=5,000$ and $6,000 \mathrm{psi}$. This agrees with the results obtained in the load measurements, in that the anchor-soil system increases in rigidity as the jack pressure increases to a certain limit and then it becomes softer as the bearing capacity of the soil is exceeded. Therefore, based on this trend, there appears to be an optimum jack pressure (in this case 3,500 psi) which makes the anchor-soil system most rigid and, permits the most efficient transmission of higher shearing stresses to the soil mass. This important effect of the jack pressure is further confirmed by compariny the maximum displacement of the anchor with the rilaximum displacement of Sensor No. 1, located 3.19 inches from the edge of the anchor. In the lower plot of Fig. G-2 it is shown that the differences in peak displacements decrease and the two curves tend to converge as the jack pressure increxses. This indicates that as the radial stress is gradually increased less slippage at the interface occurs. 

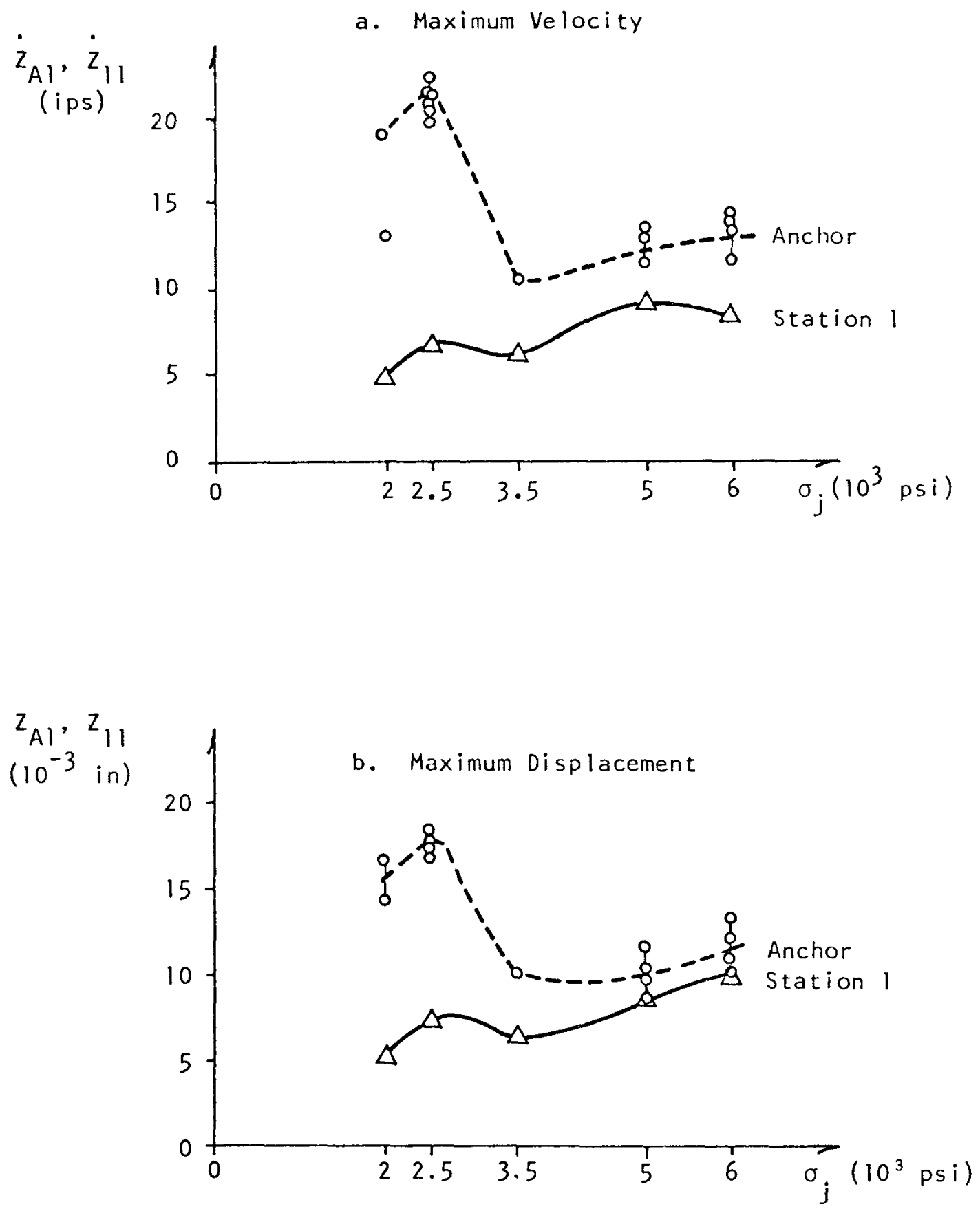

FIG. G-2 MOTIONS OF THE ANCHOR AND STATION NO. I FOR DIFFERENT JACK PRESSURES. IMPACT ENERGY $W=$ $58 \times 0.5$ ib $\mathrm{ft}$ 
These two curves, because they do not converge even at the very high pressures indicate that 100 percent coupling, for even this moderate drop height and hammer weight, is probably not possible and that some permanent displacement or anchor slippage occurs with each impact.

\section{G-3 SYMMETRY EFFECTS}

This part of the testing program was conducted to establish the importance of placing holes along a single axis or band versus on a radial pattern 120 degrees apart. Practically, a radial pattern is desirable for two reasons. First, closely spaced boreholes are less likely to become interconnected either physically or hyaraulically because they are drilled farther apart. Finally shadow effects of holes between the anchor boring and more distance holes do not become a consideration.

For data interpretation, the holes placed in a line have the distinct advantage of measuring velocities along one vertical plane, eliminating the possibility of encountering different soil types if measurements are made along different radial paths.

For this evaluation, sensors were positioned at different points radially throughout the test fill as shown in the plan Fig. F-1. In general, the borings designated A were compared with their twin sensor located in the main band. Simultaneous measurements obtained from these sensors of near equal distance from the anchor but along different radial patterns were made for this study.

Comparisons of amplitudes of two near equal distant "close in" sensors (within one foot of the anchor) produced variations in peak particle velooity amplitudes by as much as $20 \%$ at respective stations. For these close-in distances, this is not unusual and was partly due to minor differences in the travel 
distance (Appendix F) and to the fact that in this region, the measured signature is the result of two waves ( $P \& S$ waves) superimposed upon each other. Because these two waves are traveling at very different speeds (Section 5.2), each component of the wave is tending to separate rapidly producing a highly distorted signal. This distorting effect greatly influences the measured peak amplitudes, especially if the travel distances are slightly different. At greater distances, the difference in amplitudes was not this large. Comparison of decay rates of peak particle velocities with distance at two simultaneous stations each on two different radial planes indicated nearly identical values (i.e., where the "close-in" particle velocity was lower along one radial plane than another, it was always lower at other points along that same plane. This suggests that the anchor is sending consistent trains of waves that behave the same in all directions, however, the strength or amplitude of the signal leaving the anchor may be slightly different. In later production tests, simultaneous measurements of the particle velocity on different plate segments of the anchor also produced amplitudes that varied in amplitude by as much as $20 \%$. This variation is probably due to the inability to make two flat masses (the hammer and striking plate) hit evenly every time especially when these masses are usually located deep below the ground surface within a heavy drilling mud.

Fortunately, the particle velocity amplitude close-in is not a sensitive parameter, because at high strains (10-1 ${ }^{-1}$ ) the modulus change is not large. As a result, if the amplitude or the strain computed from this amplitude is off by $20 \%$ or even larger, the end result of a modulus versus strain plot is altered an essentially insignificant amount.

Comparisons of travel time between sensors equal distances from the anchor but along different horizontal lines were also conducted as also described in Appendix $F$. Since time is used 
directly to compute velocities and thus moduli, it is considered much more sensitive to minor variations. Because of distortions in the composite signals "close-in", the only repeatedly consistent point on the signature was the first arrival. At more distant recording stations (i.e., greater than about one foot from the soil anchor interface) the conventional point of zero crossing was used because the first arrival points were not aiscernable. Data of arrival times from nine different tests (36 time histories) using the same impact energy were all normalized with respect to the closest station and each point plotted with respect to distance regardless of the radial position of the station. The close-in data produced one smooth curve with all points falling on the line. A scatter in the points was almost undetectable.

For the more distant sensors, the typical variation in time at similar stations was of the order of \pm .2 msec. However, data from any successive test, when compared with other tests produced only a small change in the average velocity (determined as the slope of the line between those stations). This variation therefore, reflects that in this fill, measurements taken along different propagation paths through different soils can produce differences in the propagation velocity. It was determined from the different wave velocities measured along the single line of sensors that local soil differences could account for slight variations in velocities. These facts, therefore, lead to the conclusion that where possible for production testing, sensor holes should be accomplished along a single plane to obtain the best data possible. Where it is not possible, the radial pattern should produce reasonable results, however any local variations in the soil at random depths may produce slight time shifts which will not always produce a consistent change in velocity with strain. In such cases averaging of velocities may be required at some depths. 
Two vertical sensors were located on the plane at Elevation -3.25 feet, or 1.75 feet above the main plane of testing, and two others at Elevation -6.42 feet, or 1.42 feet below that plane. The radial aistances from the anchor were about the same as those of the sensors No. 4 and No. 10 on the band. The location of these sensors is shown in Fig. G-3.

Measurements with these sensors were accomplished to obtain a preliminary picture of the waves around the anchor, to compare with results obtained in the finite element calculations (Chapter 4 ) and to observe the distribution of deformations with time at the approximate levels of the top, middle and bottom of the anchor. Tests for this study were performed with both the hydraulic and pneumatic anchor.

For the hydraulic anchor, the vertical displacement-time histories of the top, middle and bottom stations at the radial distances of 16.39 to 16.64 inches are shown in Fig. G-4. The vertical displacements using the pneumatic anchor are presented in Fig. G-5. Sensor No. 34 failed at the beginning of the tests and the records obtained could not be used.

At the closer distances, displacements using the hydraulic anchor are large at the middle and bottom sensors and much smaller at the top. This ununiform distribution of displacements indicates that the motions of the stations correspond to the superposition of pulses from different waves propagating outward and downward from the anchor.

The displacements obtained from the pneumatic anchor appear more representative of a pure shearing condition and typical of the data obtained from the finite element analysis. In general at both the closer sensors and the more distant ones, the recorded 


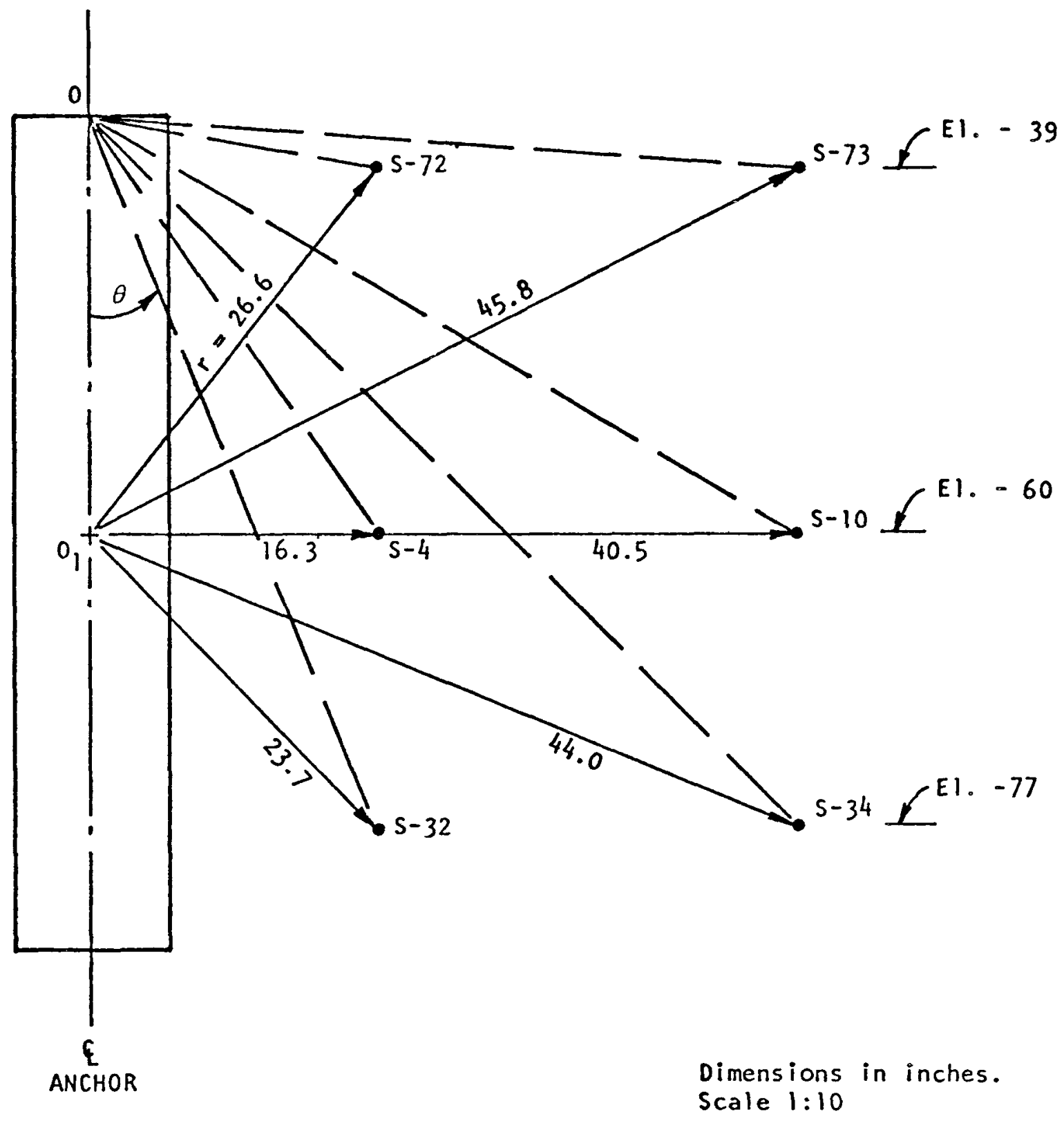

FIG. G-3 SENSORS AT DIFFERENT ELEVATIONS 


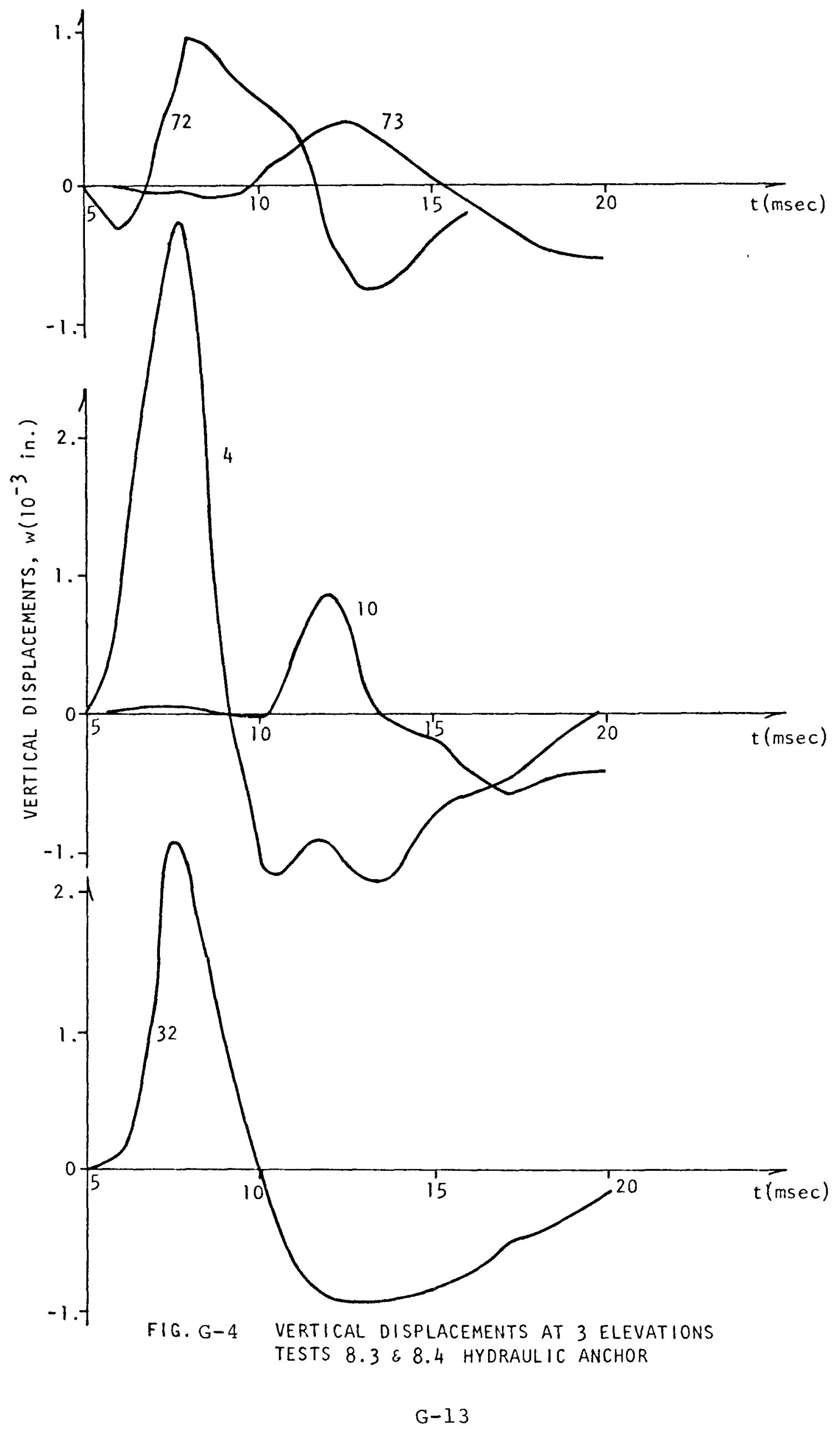




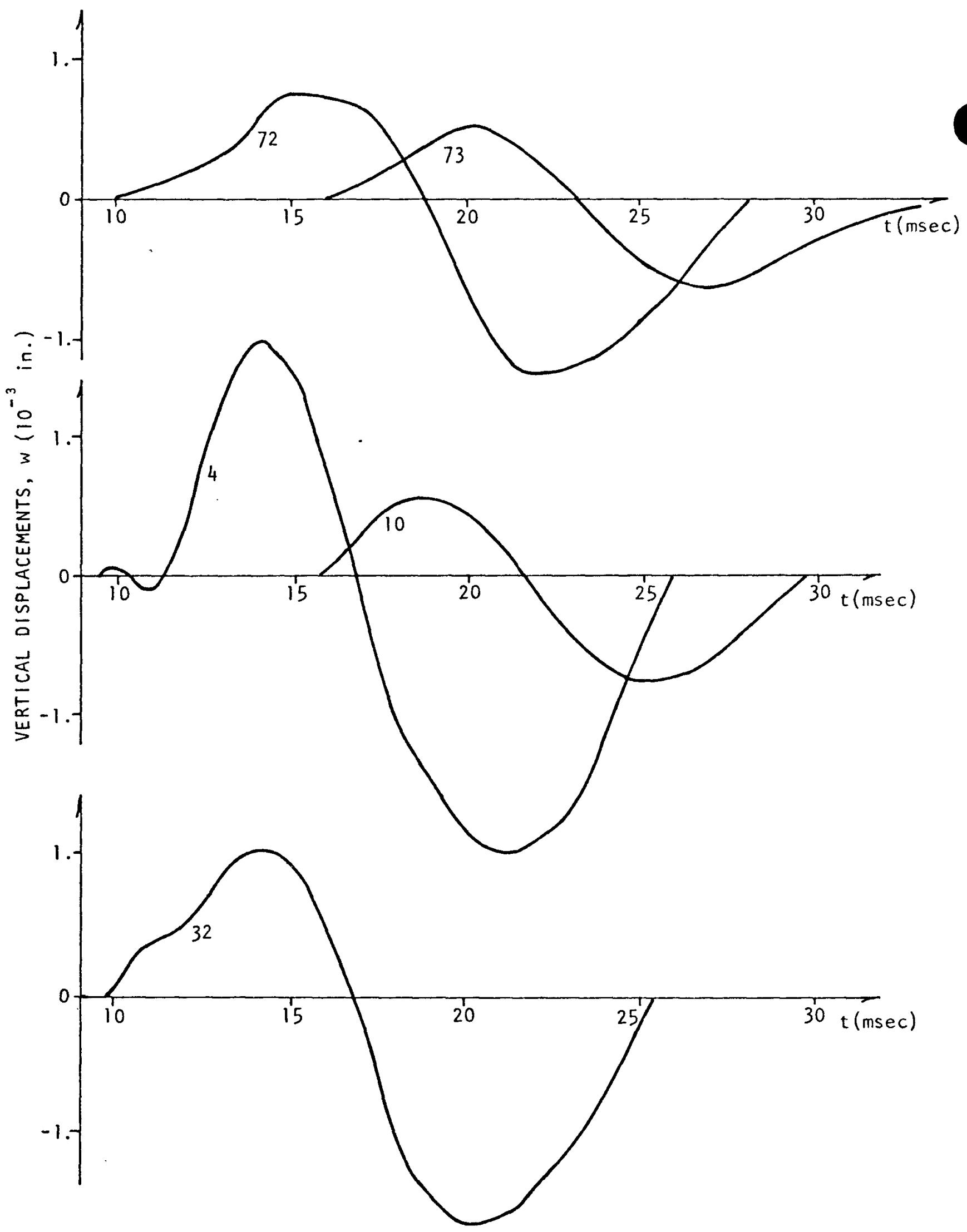

FIG. G-5 VERTICAL DISPLACEMENTS AT 3 ELEVATIONS TESTS 15.5 AND 15.6 PIVEUMATIC ANCHOR 
distribution in Fig. G-5 at five sensors is more uniform. The maximum value appears to be along the mid-depth horizontal plane. The shear velocity of the waves estimated from the travel times of the peaks is an average $416 \mathrm{fps}$ for the upper sensors, and 465 fps for the middle.

The shape of the wave front cannot be disclosed from the few measurements available. However, the fact that the displacements are not similar at corresponding times for sensors at the same radial distance, from the axis of the anchor in the closer range, indicates that the front is not exactly cylindrical or plane in this zone. In the free field the front appears as a plane shape, or sphere of infinite radius. This is suggested by the records of the sensors at the 40-inch radius which show more similar time histories.

\section{G-5 HORIZONTAL MOTION CHARACTERISTICS}

As a part of the special test program, four velocity sensors, designated 40 through 44, were placed in horizontal positions directly opposite vertical sensors 3, 5, 7 and 10 . These sensors shown in Fig. G-6 were located 13.6, 19.9, 28.4 and 40.5 inches from the center of the anchor respectively. By making simultaneous measurements from all horizontal sensors together with combined vertical and horizontal velocity measurements, horizontal response characteristics could not only be studied but horizontal and vertical components could be compared. Unfortunately, the most distant sensor (44) was damaged and did not work. In the absence of measurements from this sensor, for lack of many tests and because the remaining three horizontal sensors were in the complex distance range where the primary and main values were rapidly separating, interpretation of this data is at best. somewhat sketchy especially the determination of consistent propagation velocity trends. 


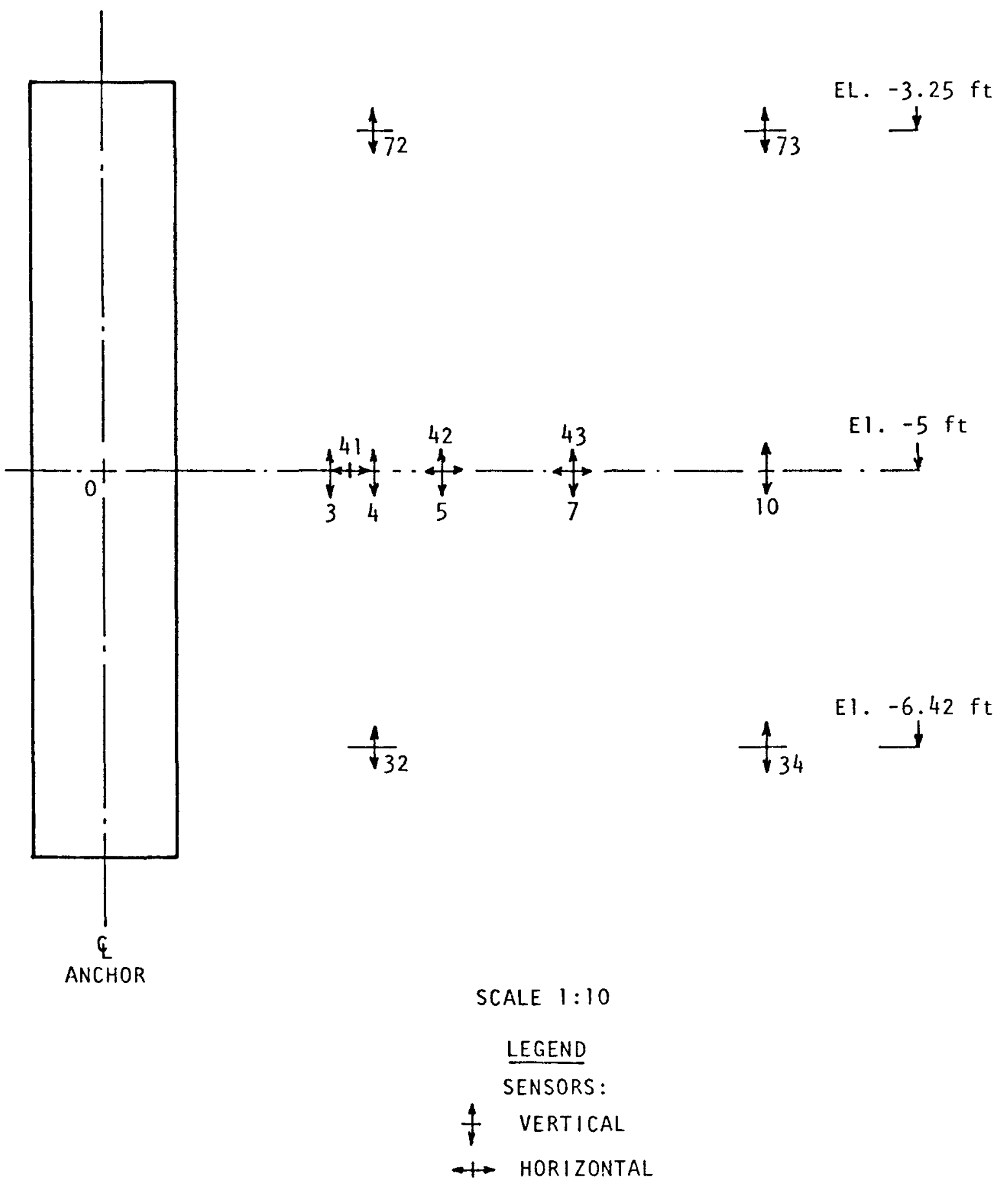

FIG. G-6 LOCATION OF HORIZONTAL AND VERTICAL SENSORS 
For studying these data, two tests consisting of six time histories for the three couples of sensors have been normalized for time shifts and are superimposed in Fig. G-7. A clear correspondence in time of the first positive peak of the vertical sensor with the first negative peak of the horizontal sensor is observed for the three couples. Also the amplitudes are about the same. This correspondence indicates a first motion downward and away from the anchor. The velocity of propagation of this first disturbance is about 1,300 fps.

The second (positive) peak of the horizontal sensors seems to match the unloading trough or second (negative) peak shown characteristically by all vertical sensors close to the anchor. since the trough in the vertical sensors is the combined result of the unloading pulse and the starting of a main loading pulse, there is not a well defined point in all three sensors. However, Fig. G-7 indicates that there is corresponaence between times of second peaks or troughs. This velocity would be in order of 950 (fps), between sensors $3-5$ or $41-42$, and 1,300 (fps) between sensors $5-7$ and $42-43$.

It is further observed in Fig. G-8 that correspondence exists between the positive peaks at time $t_{1}$ and $\mathrm{T}_{2}$ for couples 5 and 42, and 7 and 43; if the corresponding travel times and velocities are computed, using these points and including sensors 3 and 41, the disturbance is found to be traveling with a much slower velocity of the order of 480 fps.

A separate comparison of significant time points of the horizontal and vertical time histories was accomplished for a second series of tests. The resulting average wave velocities are summarized in Table G-1. Because the wave shapes are changing and separating rapidly at these distances, as indicated in Chapter 5, the computed velocities using station to station results are expected to vary; however, it is apparent that the 


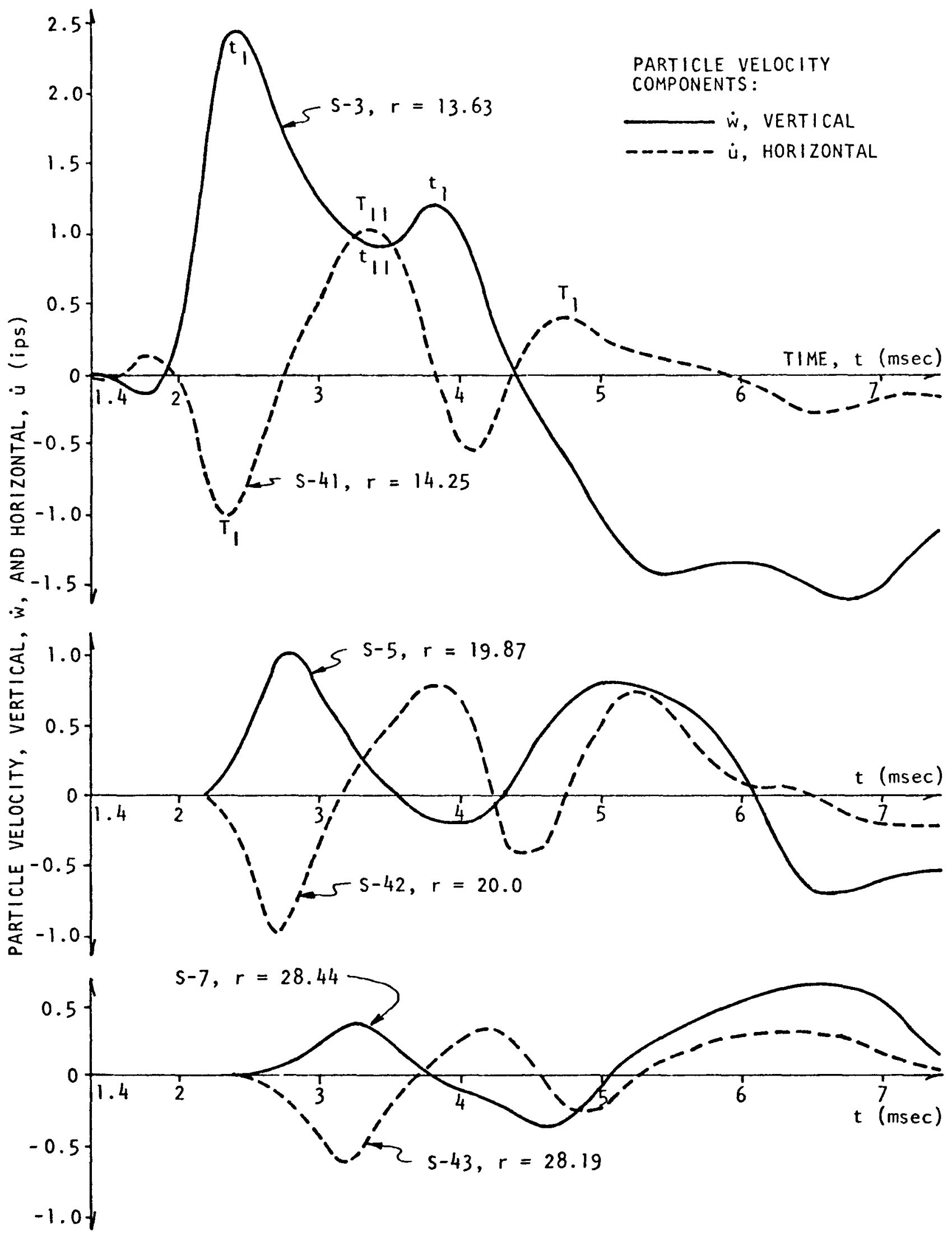

FIG. G-7 VERTICAL AND HORIZONTAL COMPONENTS OF PARTICLE VELOCITY TIME-HISTORIES.TESTS 5.5 AND 5.6. $W=58 \mathrm{lb} \times 0.5 \mathrm{ft}$, $\sigma_{j}=2,500$ psi. 


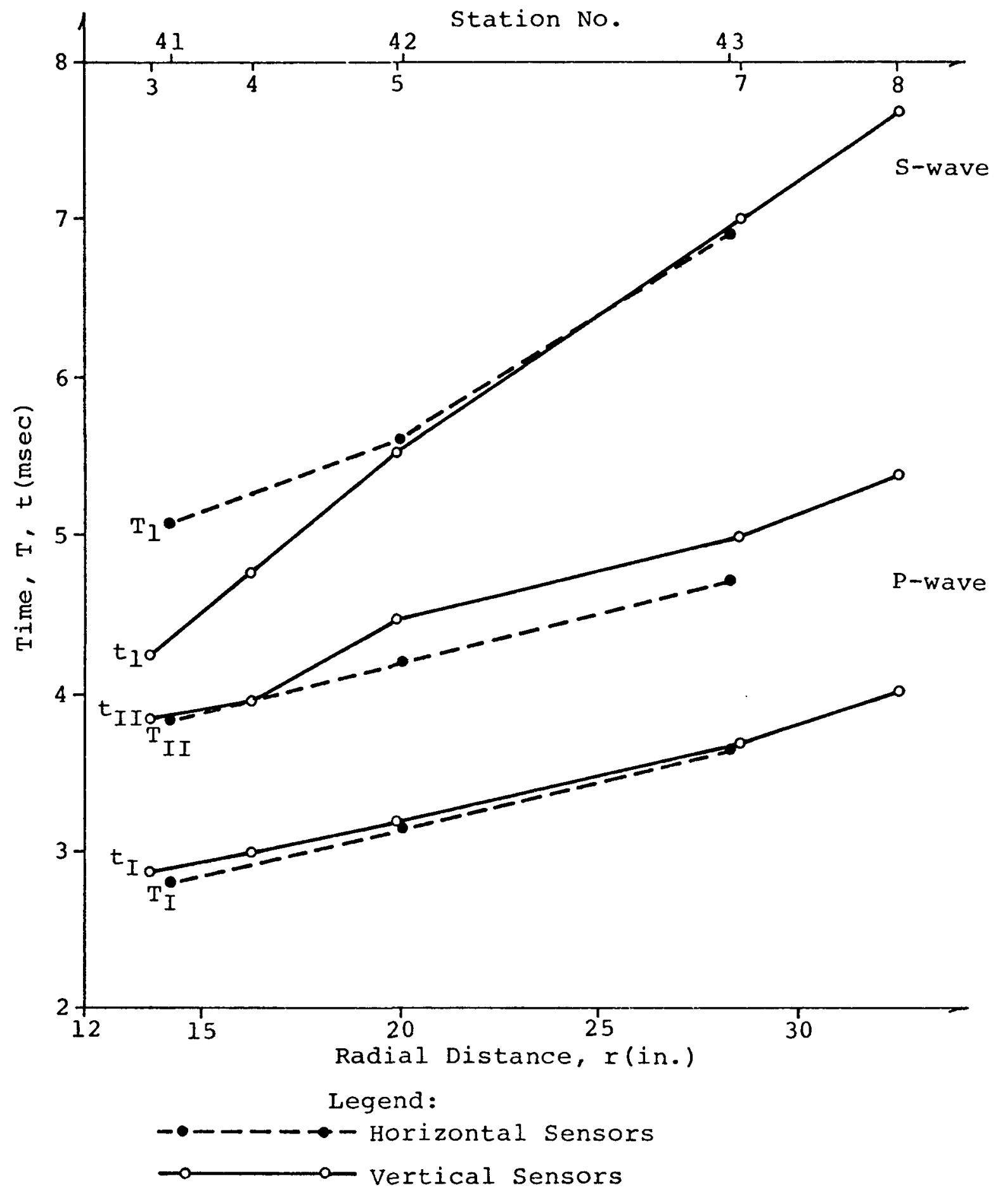

FIG. G-8 TIME - DISTANCE PLOT

FOR HORIZONTAL AND VERTICAL

SENSORS. TESTS 5.1 to 5.4 
Table G-I

Wave Velocities Computed from Tests $5.1-5.4$

\begin{tabular}{|c|c|c|c|c|c|c|c|}
\hline $\begin{array}{l}\text { Stations } \\
\text { Vertical- } \\
\quad \text { Horizontal } \\
\end{array}$ & $R_{\mathrm{T}}(\mathrm{ft})$ & $v_{t I}$ & $\begin{array}{c}\text { Wave } V \\
\mathrm{~V}_{\mathrm{TI}}\end{array}$ & $\begin{array}{c}\text { Velocities } \\
V_{\text {tII }}\end{array}$ & $\begin{array}{l}\left(\mathrm{fps}_{\mathrm{p}}\right) \\
\mathrm{V}_{\mathrm{TII}}\end{array}$ & $v_{t 1}$ & $\mathrm{~V}_{\mathrm{Tl}}$ \\
\hline $3-4$ & .218 & 1,680 & & 2,180 & & 411 & \\
\hline $4-5$ & .302 & 1,590 & & 580 & & 398 & \\
\hline $41-42$ & .479 & & 1,370 & & 1,260 & & 904 \\
\hline $42-43$ & .681 & & 1,390 & & 1,340 & & 524 \\
\hline $5-7$ & .714 & 1,400 & & 1,190 & & 476 & \\
\hline $7-8$ & .328 & 1,020 & & & & 504 & \\
\hline
\end{tabular}


first positive peak of the vertical velocity and the first negative peak of the horizontal velocity pulses travel at approximately the same velocity of 1,400 fps. It seems that the second significant peaks also travel at a similar velocity. The peaks that follow, in both pulses, travel at a much smaller velocity, which is about 450 fps. The change in velocities of these points is illustrated by the change in slopes in Fig. G-8.

Using the same points for the tests shown in Fig. G-7, the same approximate results or trends are obtained. Here the velocity of first peaks is 1,300 fps, for second peaks it is about 1,150 fps, and $400 \mathrm{fps}$ for later peaks in vertical motion and 720 fps average for horizontal motions.

Therefore, it may be concluded that the hydraulic anchor creates both $\mathrm{P}$-waves and $\mathrm{S}$-waves in this region. Based on common time points identified on both the vertical and horizontal velocity histories, these waves travel at approximate velocities of 1,300 to 1,400 and 450 to 700 fps, respectively, in this close-in region. Using these velocities in Eq. 5.1 produces values of Poisson's ratio of the order of . 35 to .4.

A series of tests with horizontal velocity measurements was also performed with the pneumatic anchor, at an internal pressure of 140 psi. The degree of coupling of this anchor with the soil was smaller than that corresponding to tests with the hydraulic anchor, because the applied effective radial stress was smaller, the contact surface was smoother and the compaction of the backill soil around this anchor was weaker. Therefore, larger slippages were observed after each test.

For the pneumatic anchor, the resulting particle velocitytime histories in horizontal and vertical direction were very ragged and difficult to interpret. However, similar differences in wave velocities, as found for the hydraulic anchor, determined 
from corresponding peaks in the pulses, were obtained in these tests.

T'he periods of the same pulses in horizontal motions have been compared for the two anchors. In general, the pneumatic anchor generates pulses about four times longer than the hydraulic anchor.

ri'he following preliminary conclusions are derived from these above observations:

a. The main source of the precursor pulses is a primary, dilatational wave which travels two to three times faster than the shear wave.

b. The physical characteristics of the originating energy source influence significantly the shapes of the pulses. The hydraulic anchor generates primary pulses of much higher amplitude than the pneumatic anchor possible due to, 1) better coupling with the soil, 2) the presence of wedges extending from the plates and 3) greater eccentric loading.

c. The significant points of the pulses which indicate unloading do not lead to a significantly different wave velocity than that computed from the positive loading peaks. This data indicates that the inelastic behavior of the soils does not have an important influence on the shape of the pulses.

Because the interpretation of horizontal motions in this program was limited to a somewhat small distance range (between 13 and 28 inches from the center of the anchor), a fuller treatment of the subject was not possible. To provide greater insight into horizontal motions and $P$-wave characteristics subsequent horizontal measurements concurrent with vertical measurements were made at several production test sites as a 
routine part of this test. This data was generally oblained at a number of depth intervals at distances of 4,8 and 16 feet from the anchor. It is anticipated that this data can be studied in future efforts.

\section{G-6 ATTENUATION OF MOTIONS}

The components of particle motions generated by impulse tests, attenuate with distance from the source. This attenuation is caused by geometrical spreading of the energy of the waves and by hysteretic damping. The geometrical effect (sometimes called geometrical damping), as recorded by an arrangement of receiving stations, is dependent upon the shape of the wavefront and the direction in which it propagates, relative to the orientation of the stations. The hysteretic damping is a property of the material through which the waves propagate and it depenas upon the strain amplitude of the motions.

Fig. G-9 summarizes the results of three series of tests performed with the same impact energy of $58 \mathrm{lb} \times 0.5 \mathrm{ft}$. The jack pressure was different, in each series: 2,000, 3,500 and $5,000 \mathrm{psi}$. Both $\mathrm{P}$ and $\mathrm{S}$-pulse peak particle velocities are plotted versus radial distance in the figure.

It is first observed that higher jack pressures lead to higher particle velocities throughout the soil mass. This is consistent with the fact that higher jack pressures produce better coupling of anchor and soil, and therefore, more efficient transmission of energy from the impact source into the soil.

Second, the attenuation rates for $\mathrm{P}$-pulses are higher than for $\mathrm{S}$-pulses. In effect, the slopes of the log-log lines representatives of the general trends of attenuation for $\mathrm{P}$ and S-particle velocities are, approximately, -2.4 and -1.4 , respectively. Because of the superposition of pulses in the 


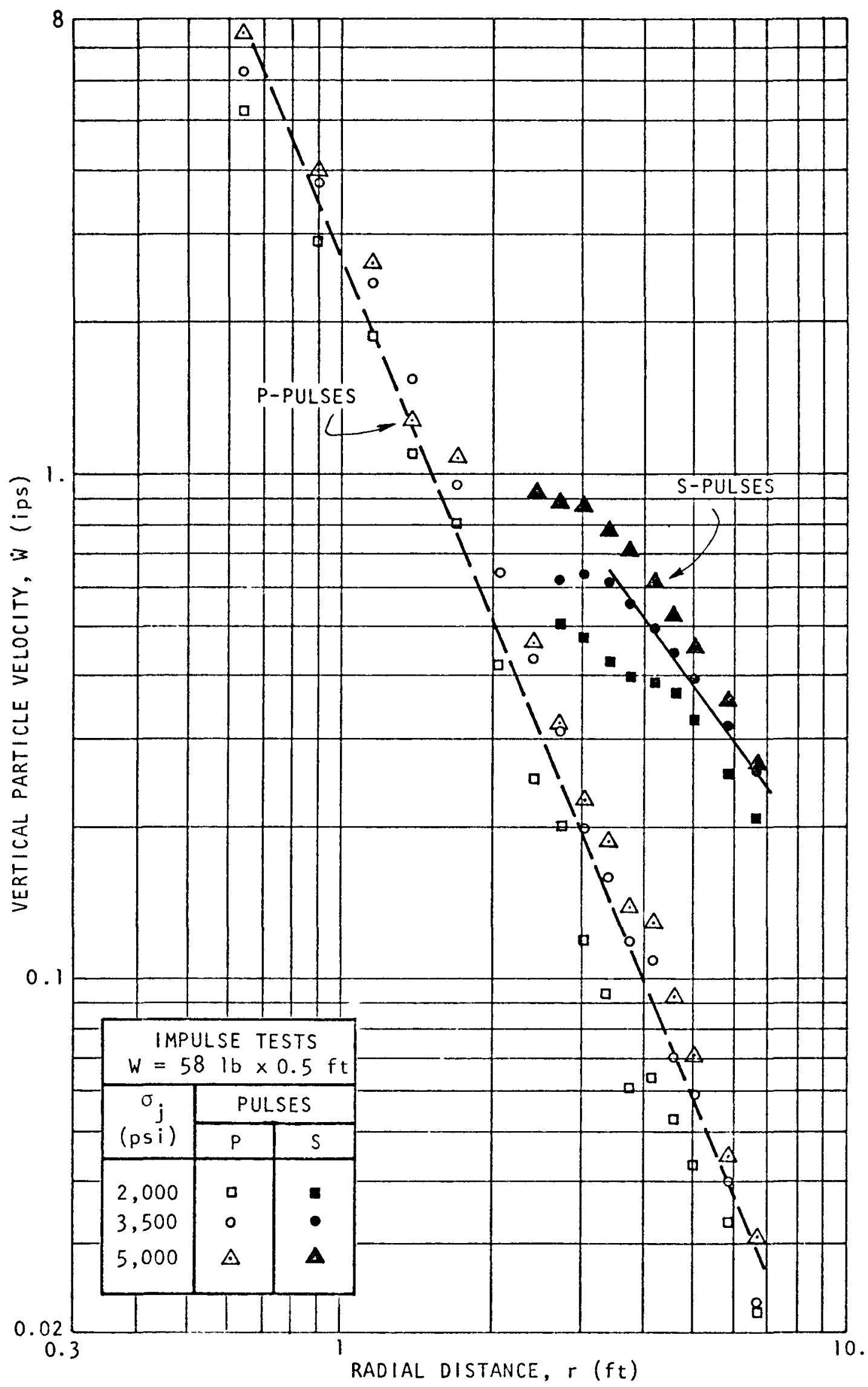

FIG. G-9 PARTICLE VELOCITY ATTENUATION - P E S PULSES 
close range, these rates of attenuation are only approximate. The differences in the rates between $P$ and $S$ pulses may be due to a different geometrical pattern of propagation of the waves. $P$ waves traveling in an inclined direction, outward and downward from the source, will produce decreasing vertical components of motions, as distance increases, in stations aligned on a mid-depth horizontal plane. S waves may travel more preferably in a horizontal direction, outward from the source, thus generating predominant vertical components of motion in the middepth plane.

Hysteretic damping can be estimated from the rate of attenuation of the waves after deducting the effect of geometrical damping from the total rate of attenuation. At this point, a method for converting these results to a useful form for estimating material damping has not yet been established. This procedure, however, introduces one possible avenue for future work in evaluating damping in situ. 